\title{
Cavitation and shock wave effects on biological systems
}

\author{
Dissertation \\ zur Erlangung des Doktorgrades \\ der Mathematisch-Naturwissenschaftlichen Fakultäten \\ der Georg-August-Universität zu Göttingen
}

vorgelegt von

Bernhard Wolfrum

aus Bonn

Göttingen 2004 
D7

Referent: Prof. Dr. Werner Lauterborn

Korreferent: Prof. Dr. Dirk Ronneberger

Tag der mündlichen Prüfung: 10.2.2004 


\section{Contents}

$\begin{array}{lll}1 & \text { Introduction } & 7\end{array}$

2 Cell mechanics $\quad 11$

2.1 Cell permeabilization and molecular delivery . . . . . . . . . . . . 11

2.2 Cell adhesion . . . . . . . . . . . . . . . . . . . 14

3 Shock waves and bubble dynamics 18

3.1 Shock waves . . . . . . . . . . . . . . . . . . . . . . 18

3.1.1 Lithotripsy: A shock wave application . . . . . . . . . . 18

3.1.2 Theoretical description of shock waves . . . . . . . . . . . 21

3.2 Spherical bubble dynamics . . . . . . . . . . . . . . . . . 26

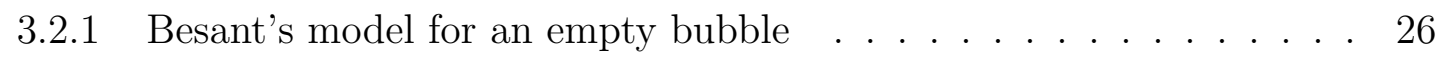

3.2 .2 Rayleigh-Plesset model . . . . . . . . . . . . . . . . . . . 27

3.3 Aspherical bubbles . . . . . . . . . . . . . . . . . . . . 29

4 Shock wave induced interaction of microbubbles and boundaries 32

4.1 Methods . . . . . . . . . . . . . . . . . . . . . . 33

4.1.1 Shock wave creation and measurement . . . . . . . . . . . 33

4.1 .2 High-speed microscopy . . . . . . . . . . . . . . . . . . 36

4.1.3 Numerical modeling of the bubble dynamics . . . . . . . . . . 38

4.2 Bubble-boundary and bubble-bubble interaction . . . . . . . . . 38

4.3 Particle disruption . . . . . . . . . . . . . . . . . . 50

4.4 Conclusion . . . . . . . . . . . . . . . . . . . . . 51

5 Pressure wave excited contrast agent bubbles in the vicinity of cells $\quad 54$

5.1 Materials and methods . . . . . . . . . . . . . . . . 54

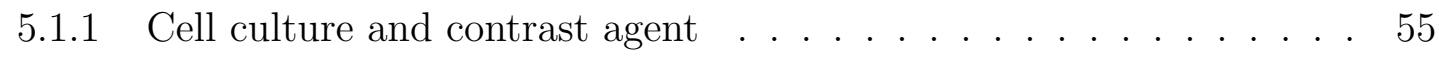

5.1 .2 Pressure excitation of contrast agents . . . . . . . . 5 56 
5.2 Bubble dynamics and cell deformation . . . . . . . . . . . . . . . . . 57

5.3 Conclusion . . . . . . . . . . . . . . . . . . . . . . 71

6 Shock wave and cavitation generation after impact of high-speed projectiles on tissue substitut 6.1 Experimental setup . . . . . . . . . . . . . . . . . . . 72

6.2 Impact of high-speed bullets on aqueous substances and secondary effects . 74 6.2.1 High-speed bullet impact on gelatin and water . . . . . . . . . . . . 74

6.3 Conclusion . . . . . . . . . . . . . . . . . . . . . . . 93

7 Interactions of adherent cells with shock wave induced cavitation $\quad 94$

7.1 Materials and methods . . . . . . . . . . . . . . . . . . . . 94

7.1.1 Cell culture . . . . . . . . . . . . . . . . . . 94

7.1.2 Viability stain . . . . . . . . . . . . . . . . 95

7.1.3 Transient membrane permeabilization . . . . . . . . . . . . . 96

7.1.4 Shock wave generation and microscopic observation . . . . . . . . 96

7.2 Cavitation inception . . . . . . . . . . . . . . . . . . . . . . . . 99

7.3 Cell displacement and detachment . . . . . . . . . . . . . . . . . . . . . 101

7.4 Molecular uptake and cell viability . . . . . . . . . . . . . . . . . 110

7.5 Discussion . . . . . . . . . . . . . . . . . . . . . . . . . . . . . 114

7.6 Conclusion . . . . . . . . . . . . . . . . . . . . . 116

$\begin{array}{llr}8 & \text { Outlook } & 117\end{array}$

$\begin{array}{ll}\text { Acknowledgement } & 120\end{array}$

$\begin{array}{ll}\text { Bibliography } & 121\end{array}$ 


\section{Summary}

This thesis investigates the effects of cavitation and shock waves on systems of biological or medical relevance. Its main focus lies on high-speed optical observations of cavitation bubble activity after pressure wave excitation and the resulting effects on cells, which comprise cell damage, transient membrane permeabilization, and the detachment of cells adhering to a substrate. The theoretical background and possible applications of shock waves, bubble dynamics and certain aspects of cell mechanics are given in Chapters 2 and 3 .

In the experimental part of this work different methods have been used to create and excite bubbles. In a first approach described in Chapter 4 preexisting bubbles in the vicinity of rigid boundaries are excited by a laser induced shock wave. The effects of neighboring bubbles and rigid boundaries on bubble dynamics are observed with microscopic high-speed recordings. The bubble dynamics is calculated numerically using the Keller-Miksis model and results are compared with the experimental radius time curves.

A similar microscopic high-speed imaging setup is then used to investigate the dynamics of bubbles in the vicinity of cultured rat kidney fibroblast cells (NRK-cells). The bubbles have been formed from contrast agents (Levovist) for medical ultrasound imaging after spark-induced pressure wave application (Chapter 5). Microscopic high-speed images show rapid bubble expansion and collapse. Even at moderate peak negative pressure amplitudes of less than $1 \mathrm{MPa}$ the contrast agent bubbles have been observed to expand to more than 30 times their original radius and to rupture cells upon collapse. The bubble dynamics of excited contrast agent bubbles show reasonable agreement with a spherical bubble model during the first oscillation cycle.

The destructive behavior of pressure excited bubbles might also be important in ballistic injuries. In this context the effects of high-speed projectiles on tissue substitutes have been investigated (Chapter 6). While low-speed bullets can pass material with high water contents without fragmentation, high-speed projectiles disintegrate shortly after entering the tissue substitute. The impact of the bullet generates a shock wave, which travels 
through the medium. Upon reflection at low acoustic impedance boundaries, the shock wave becomes a tensile wave generating cavitation clouds in its wake. The cavitation bubbles might cause secondary damage inside the tissue farther away from the bullet and its fragments.

The last experiment described in Chapter 7 deals with the interactions of lithotripter generated shock waves with cancer cells from the uterus epithelium (HeLa-cells) adhering to a substrate. It is shown that shock waves permeabilize adherent cells in vitro through the action of cavitation bubbles. The bubbles are formed in the trailing tensile pulse of a lithotripter generated shock wave where the pressure drops to a minimum of approximately $-4 \mathrm{MPa}$. Upon collapse of cavitation bubbles a strong flow field is generated, which accounts for two effects: The detachment of cells from the substrate and the temporary opening of cell membranes followed by molecular uptake.

Finally an outlook of progress in new techniques involving cavitation effects on cells is given in Chapter 8. 


\section{Chapter 1 Introduction}

The formation and vivid activity of bubbles in liquids is called cavitation. Bubble formation in liquids is a phenomenon that is encountered in manifold situations. Be it in a boiling soup, in our blood after rising too fast from a scuba dive, during the use of high-speed torpedoes, or in a pub drinking our favorite beer: bubbles are always present, sometimes highly desired as in the case of the beer and sometimes unrequested especially during the scuba dive. The term cavitation usually describes bubble formation under violent conditions like the rupture of liquids or strong expansion of preexisting bubble nuclei due to large negative pressures. These bubbles often show very destructive characteristics to boundaries in their vicinity.

Strong interest in a particular research field usually arises when the object of investigation either poses a problem to an existing application or promises to yield a new application. Both of this happened to cavitation research in the naval industry. At the end of the 19th century it was discovered that the maximum speed of steam boats was limited by generation of cavitation bubbles at the ship's propellers [1]. Propeller blades moving at high velocity in the liquid decrease the pressure on them. If the pressure drops below vapor pressure bubbles are formed. This typically happens to the forward side of poorly designed propellers near the leading edge at the tip of the blades. The generated bubbles increase the slip and thereby reduce the thrust of the propulsion system. Into the bargain, propeller blades display strong erosion due to bubble activity. To overcome these two problems, cavitation bubbles and their damage potential to propeller blades were investigated. The minimization of cavitation and erosion is still crucially important not only for propeller design of marine vessels but in many technical applications. More recently the idea of supercavitating torpedoes, which can surpass the speed of their conventional counterparts, have stirred the interest of the naval industry. Supercavitating torpedoes or other supercavitating projectiles only touch the liquid at their tip and are enclosed by a 
bubble elsewhere. This reduces the drag imposed on the torpedo and facilitates to achieve higher maximum velocities [2].

Besides being of technical relevance, cavitation bubbles allow to study interesting physical phenomena. For example, a bubble trapped in a sound field can be stimulated to emit a flash of light every oscillation cycle [3]. This process is called stable sonoluminescence. Excited bubbles display nonlinear oscillations with extreme conditions during their repetitive contraction phase. Upon collapse the gas or vapor inside a bubble is compressed and high temperatures and pressures can be achieved. For instance, an argon bubble with $5 \mu \mathrm{m}$ ambient radius driven sinusoidally with pressure amplitudes of $0.13 \mathrm{MPa}$ at $20 \mathrm{kHz}$ has been estimated to reach $20000 \mathrm{~K}$ upon collapse [4]. Even higher temperatures might be reached if shock waves inside the bubble contribute to the heating process [5]. Molecular dynamic simulations of bubbles under similar conditions (initial radius: $4.5 \mu \mathrm{m}$, driving pressure amplitude: $0.13 \mathrm{MPa}$, and frequency: $26.5 \mathrm{kHz}$ ) predict temperatures on the order of $50000 \mathrm{~K}$ [6]. The extreme conditions during bubble collapse are also interesting for sonochemists, who investigate chemical reactions under the influence of sound $[7,8]$. The rapid nonlinear dynamics of bubbles is challenging for both experimentalists and theoreticians who work in the field of cavitation. Although various aspects of cavitation are still unknown, complex theoretical models describing spherical and sometimes also aspherical bubble dynamics $[9,10,11,12]$ have been developed and improved since the fundamental work on bubbles by Lord Rayleigh in 1917 [13]. Also high-speed imaging techniques to capture the fast bubble dynamics in different experimental setups have contributed to the understanding of many cavitation effects in the last years $[14,15,16,17,18]$.

The generation methods of cavitation are manifold. Usually it is distinguished between tension induced cavitation and other methods of energy depositions in the liquid [18]. Bubbles generated by fluid flow or sound fields belong to the former group and are called hydrodynamic and acoustic cavitation, respectively. The latter group comprises bubbles created by electrical sparks, focused laser light, heat, chemical explosions, and elementary particles. Although the generation mechanisms of cavitation bubbles are very different the dynamics of differently created bubbles may still be similar. It is therefore possible to investigate spark or laser generated bubbles in a controlled setup and transfer certain aspects of the results to tension induced cavitation. This has been done, in particular, to study erosion effects at rigid boundaries without creating hydrodynamic cavitation in an experimental setup.

The use of medical treatment and diagnostic techniques, which have the potential of causing bubble formation inside a patient, raised the question of possible effects of cav- 
itation on biological systems. For example extracorporeal shock wave lithotripsy, the destruction of calculi by focusing shock waves inside a patient, is known to be accompanied by cavitation. Also the use of contrast agents in ultrasound imaging techniques lowers the threshold to create cavitation inside the blood vessels. Optical cavitation may occur during laser surgery by plasma generation for instance in ophthalmology [19]. On one hand it is important to investigate cavitation effects in medical applications with respect to possible hazards to the patient and possible interference with the desired treatment or diagnosis. On the other hand, research on the interactions of cavitation with biological systems may help to improve or facilitate new applications in medicine or biology. For instance drug delivery due to the transient permeabilization of cells or transport vesicles by ultrasound or shock waves may be caused by cavitation effects. A better understanding of the interactions of cavitation with cells might therefore contribute to gene therapy and other drug delivery approaches. Next to medical applications bubble-cell interactions might also be important in ultrasonic cleaning devices and certain types of ballistic injuries. The latter will be shown in Chapter 6 . 
Kap. 1 Introduction 


\section{Chapter 2}

\section{Cell mechanics}

Cells are the basic units in every biological system. To understand cavitation effects on biological systems it is therefore important to investigate the interactions of bubbles with cells. The experiments described in Chapter 5 and 7 were performed to directly observe interactions of oscillating bubbles with cells in vitro. These experiments show that mechanical interactions of cavitation bubbles with cells can lead to cell destruction, cell permeabilization, and cell detachment. The following sections deal with those effects and their possible importance on medical or biological applications. However, it should be noted that the experiments are performed in vitro and therefore the results cannot be applied to in vivo conditions without further consideration.

Some properties of biological tissues, i.e. cells and intercellular medium, can be modeled by soap, gelatin, or even pure water [20]. The experiments of Chapter 6, where the action of ballistic shock and tensile waves and the generation of cavitation is observed, are thought to transfer similarly to realistic tissue.

\subsection{Cell permeabilization and molecular delivery}

All cells are enclosed in plasma membranes consisting primarily of lipids and proteins. The lipids of a membrane are arranged in a double layer, which is held together mainly by hydrophobic interactions of the nonpolar hydrocarbon chains. Polar head groups of the lipids face towards the interior of the cell called cytosol and towards the extracellular medium. The lipid bilayer can be viewed as a two-dimensional fluid, since it is only a few nanometers thick and the lipid molecules are able to move about in the membrane. Besides keeping the entity of the cell, the plasma membrane also controls the transport 
between the extracellular and intracellular medium. While the lipid bilayer is relatively impermeable to polar molecules, proteins incorporated in the membrane act as pumps or channels to amplify or decrease ionic gradients across the membrane. The control of molecular transport across the membrane is crucial for cell survival. However, for medical applications it is often necessary to circumvent the cells own control mechanisms and to insert normally membrane impermeable molecules into the cell. Such a procedure is called molecular or drug delivery. A special kind of drug delivery is the insertion of genes into the cell. If the DNA-sequence reaches the nucleus it can be expressed by the cell's own machinery into proteins. Nowadays there exist several different approaches to facilitate gene delivery [21]. The choice of a particular method depends on the target system. In particular, it is discerned between molecular delivery inside a patients body (in vivo) or in a cell culture (in vitro). In gene therapy a lot of clinical trials are based on viral delivery systems, called viral vectors to incorporate the desired DNA into host cells [21]. These approaches use to advantage that during evolution viruses have perfected the ability to introduce their genetic material into cells. They often do this by a process called receptormediated endocytosis, where the virus first binds to a receptor on the cell surface and is afterwards engulfed by the plasma membrane. The segment of the membrane surrounding the virus pinches off and a vesicle containing the virus remains inside the cell. In clinical trials the viral genome is previously manipulated to remove disease-causing genes and insert the desired therapeutic genes. Up to now most clinical trials for gene therapy use either retro- or adenoviral vectors, the former being a class of viruses, which can create double-stranded DNA copies of their RNA genomes, like the human immunodeficiency virus (HIV). These copies can be integrated into the chromosomes of host cells. The adenovirus is a class of viruses with double-stranded DNA genomes. It contains a protein capsid as an outer surface and may cause respiratory, intestinal, or eye infections in humans. A problem with the use of viral vectors lies in the response of the immune system to the foreign proteins. Therefore, immune reactions have to be suppressed during therapy, which may cause problems especially after multiple treatments. In addition the preparation of a viral vector is time consuming and expensive and also concerns are remaining that, once inside a patient, it may recover its ability to cause disease [22].

Another possibility for gene therapy approaches is the use of nonviral delivery systems. For example, cationic lipid vesicles can act as carriers for genetic material [23, 24, 25]. In this case the genes are brought into the cytosol after membrane fusion of vesicle and cell. The advantage of such a system lies in the lack of an immune response. However transfection rates are usually small compared to viral approaches. A standard technique 
for gene transfection in vitro is the calcium phosphate mediated procedure. A precipitate containing DNA and calcium phosphate is taken into cells by an endocytosis mechanism. Although this method works well for many adherent cell lines, it is hampered by cytotoxic side effects and cannot be well applied in vivo [26].

The prevalent physical method for drug delivery is electroporation. In this method cell membranes are disrupted by a strong external electric field leading to a transient increase in diffusive permeability [27]. The electric field induces the reorientation of lipid molecules to form hydrophilic pores in the membrane. During the lifetime of the pores extracellular molecules can diffuse into the cell. After the electrical pulse is terminated, the membrane permeability decreases back to its original value due to the closing of the pores. Relaxation times are in the order of milliseconds to seconds [28] and depend on the size and distribution of the pores, larger pores being more stable than smaller ones. If very high or long voltage pulses are applied, the membrane may not recover at all, which results in a permanently damaged cell. Electroporation is mainly used for cell suspensions, which are filled between two flat electrodes. However, Lundqvist et al. [29] also developed a method to target single cells using carbon fiber microelectrodes. Another physical method for gene delivery is the so called gene gun. In this technique DNA-coated microparticles are shot directly into the cells [30]. The particles are thereby accelerated for example by applying pressurized helium [31]. Although this technique is mainly used for the transformation of plant cells, it can be used for drug delivery to other cell types as well. Membranes can also be permeabilized by optical means. It is distinguished between two types of optical cell permeabilization: optoinjection and optoporation. During optoinjection low energy femtosecond laser pulses are focused on a single cell. It is assumed that the membrane is permeabilized by chemical interactions with a low density plasma, which is created in the focal spot of the laser. This technique has been successfully used to transform adherent neurons with high efficiency [32]. Optoinjection promises to be the method of choice if selectivity is required. From a network of neurons it is possible to introduce genes into a single cell without altering its neighbors. Another approach for cell permeabilization and drug delivery also based on optical techniques is the so called optoporation [33]. If the intensity in the focal spot of a laser surpasses the breakdown limit of the liquid it generates a plasma. A shock wave is emitted and a vapor bubble originates at the focal spot. Due to the outward acceleration of the surrounding fluid, the bubble first expands up to maximum radius determined by the initial conditions. Afterwards it contracts and emits a second shock wave during the final stage of the collapse. Cells in the vicinity of an optical breakdown can be transiently permeabilized. The permeabilization is probably 
caused by shear stress $[34,35,36]$, which is either generated by the outgoing shock waves or by a strong flow field during the rapid bubble dynamics.

Of particular interest for possible in vivo application is the permeabilization of cell membranes by acoustic means, called sonoporation. Gambihler et al. have shown that application of lithotripter shock waves can facilitate the uptake of usually membrane impermeable molecules into L1210 mouse leukemia cells in vitro [37]. Since then many studies were performed to assess membrane poration and molecular delivery after ultrasound and shock wave exposure in vitro $[38,39,40,41,42,43,44,45,46,47,48,49,50,51,52,53,54]$ and in vivo $[55,56,57,58,59]$. Drug delivery methods based on acoustical approaches can take advantage of the ability to focus acoustic waves inside the body without the need of surgery. Nowadays shock waves are already frequently focused inside patients to disintegrate renal calculi. New drug delivery applications can make use of techniques developed in lithotripsy. Although intensive research in quantifying sonoporation has been performed in the last years, the mechanisms, which are responsible for the permeabilization of cells during shock wave and ultrasound applications are not yet fully clarified. However, similar to optoporation they are probably based on direct interactions between the pressure wave and the cell or, as will be addressed in Chapter 7 , on the generation of cavitation bubbles.

\subsection{Cell adhesion}

Cell adhesion lies at the heart of many biological phenomena of multicellular organisms. For example it is essential to the cohesion of tissue, cell migration, the formation of organs during embryological development, wound healing, immune response after inflammation, and pathological tumor invasion $[60,61]$. Considering the diversity of these phenomena it is perspicuous that the adhesion process is variable for different cell types or even the same cells under different conditions. Epithelial cells of the intestine for example connect each other by tight adhesion belts, which act as a barrier for many molecules. The cells are arranged in a sheet-like structure separating the apical surface facing the lumen from the basal surface of the cells, which are in turn attached to a network of extracellular macromolecules, the extracellular matrix. In general, one can distinguish between cell-cell adhesion and the attachment of cells to the extracellular matrix. In both cases however, the attachment of cells is mediated by linker proteins [60]. An important family of transmembrane linker proteins for cell-cell adhesion are the cadherins. Members of 


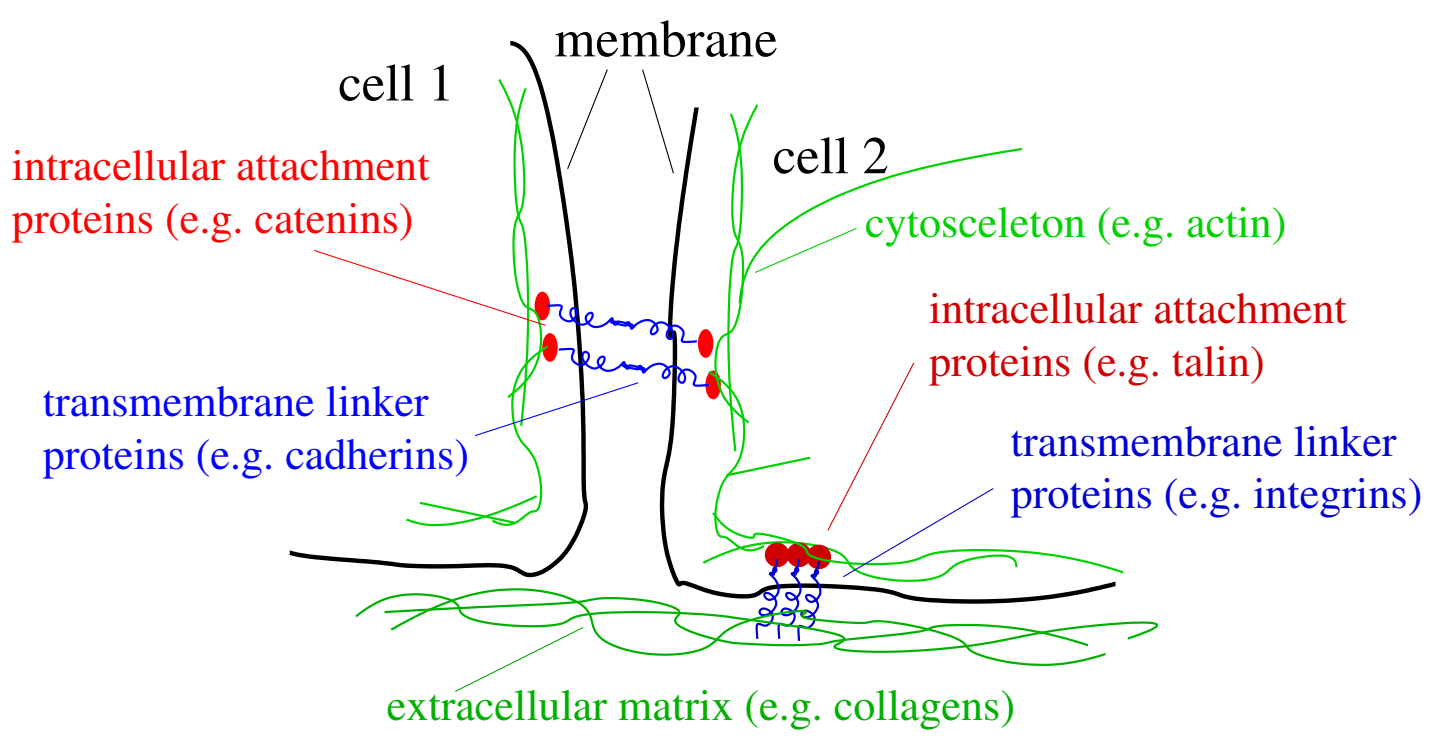

Figure 2.1: Schematic drawing of cell-cell and cell-matrix adhesion. The transmembrane linker proteins, adhering either to another cell or the extracellular matrix, are connected via intracellular attachment proteins to the cytoskeleton.

this family usually display homophilic binding, where the same type of molecule acts as receptor and ligand on two adjacent cell surfaces. Cadherin attachment depends strongly on the presence of $\mathrm{Ca}^{2+}$ making it possible to dissociate these bindings in cell culture by deprivation of divalent cations. However, other cell-cell adhesion molecules, for example from the immunoglobulin superfamily exist, which are $\mathrm{Ca}^{2+}$ independent. Another very important transmembrane linker protein family for cell adhesion are the integrins. They consist of a heterodimer of associated $\alpha$ - and $\beta$-units and are divided into subfamilies based on sharing equal $\beta$-units with varying $\alpha$-units. Most integrins bind to components of the extracellular matrix like collagen or fibronectin and are also dependent on divalent cations. If cells are supposed to form robust attachment at cell-cell or cell-matrix adhesion sites it is not sufficient for adhesion molecules to simply connect to the plasma membrane of the cell. In fact, to generate a firm junction the cell adhesion molecules must be linked to the stable structure of the cytoskeleton inside the cell. This is achieved by intracellular attachment proteins, which connect the intracellular part of transmembrane linker proteins to either actin or intermediate filaments of the cytoskeleton; see the sketch in Fig. 2.1. Often several adhesion molecules work together to create focal contact junctions at a specific spot. Through the junctional attachment sites forces can be mediated from cells to an extracellular matrix and vice versa. For example fibroblast cells in culture generate tension on the intracellular filamentous network, probably by the action 
of molecular motors, which is transmitted to the attached substrate [61]. In return, the extracellular matrix can influence the arrangement of the cytoskeleton affecting cell shape. Cell adhesion molecules do not merely act as static mechanical connectors. Integrins on red blood cells for instance have to be activated before they can mediate cell adhesion. Also force-transmitting cell adhesion molecules like integrins may act as sensors, which transduce information of external mechanical loads into biochemical signals. As a result the cell responds with changes in gene expression affecting for example cell growth and again cell adhesion.

In culture, cells adhering to a substrate display the typical spreading as seen in Fig. 2.2 (a). When adhesion molecules are inhibited, the cells detach and adopt a round shape (Fig. 2.2 (b)). Facilitation of cell detachment is obtained if the cytoskeleton of
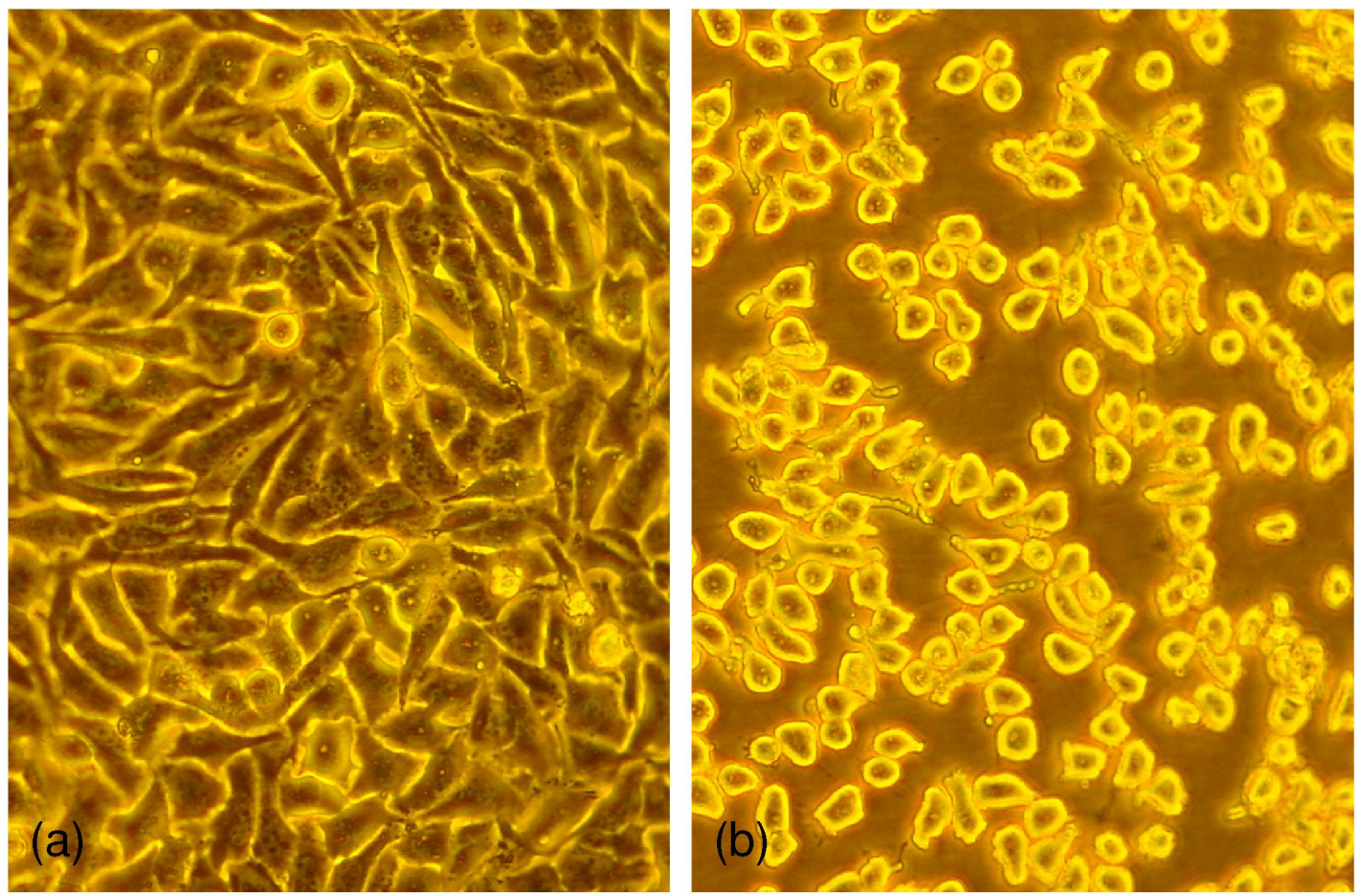

Figure 2.2: Epithelial cancer cells on a polystyrol substrate (a) before and (b) after treatment with trypsin/EDTA. Trypsin/EDTA inhibits cell adhesion. Consequently the cells round up and detach from the substrate as seen in (b).

cells is depolymerized, depriving adhesion molecules of their intracellular anchors [60]. External forces such as shear flow along the substrate can also cause cell debonding. For a given cell, detachment occurs if the shear stress reaches a certain threshold. The threshold probably depends on the contact surface and the number and type of adhesion bonds. 
Decave et al. [62] describe the kinetics of shear flow induced detachment in a peeling model where adhesive bonds break progressively with time, starting at the edge of the cell facing the flow. This velcro-like detachment procedure allows for cell detachment at relatively low shear stresses compared to instantaneous whole cell detachment. Violent shear flow however, may cause rapid cell detachment. Examples of this, caused by the action of cavitation bubbles, are presented in Chapter 7 . 


\section{Chapter 3}

\section{Shock waves and bubble dynamics}

\subsection{Shock waves}

Shock waves are characterized by a steep pressure front whose length scale is in the same order as the mean free path for particles of the medium. They usually appear because of violent initial conditions like explosions or supersonic movement of objects or due to steepening of high amplitude pressure waves caused by nonlinear wave propagation. The characteristics of a shock wave depend on the properties of the supporting medium. In gases, shock waves with amplitudes of about hundred atmospheres are considered strong. Due to the high compressibility of gases small pressure variations induce large changes in the density of the medium. The compression is accompanied by a strong temperature rise. In less compressible media such as most solids and liquids, a shock wave with $10 \mathrm{MPa}$ is still considered weak because only small density changes are induced and also the temperature within such a shock wave region remains fairly constant [63].

With the invention of missiles and aircraft which exceed the speed of sound in air, shock wave generation in gases has become a field of intensive research. However, up to now no marine vessels exist, which are able to surpass the speed of sound in water. Nevertheless, interest in liquid bound shock waves has been stirred especially by their possible use in ultrasonic cleaning and medical applications. Furthermore, research on underwater explosions has contributed to the understanding of shock waves in liquids [64].

\subsubsection{Lithotripsy: A shock wave application}

The most prevalent use of shock waves in medicine is the disruption of renal and other calculi. In extracorporeal shock wave lithotripsy (ESWL) the shock waves are created out- 


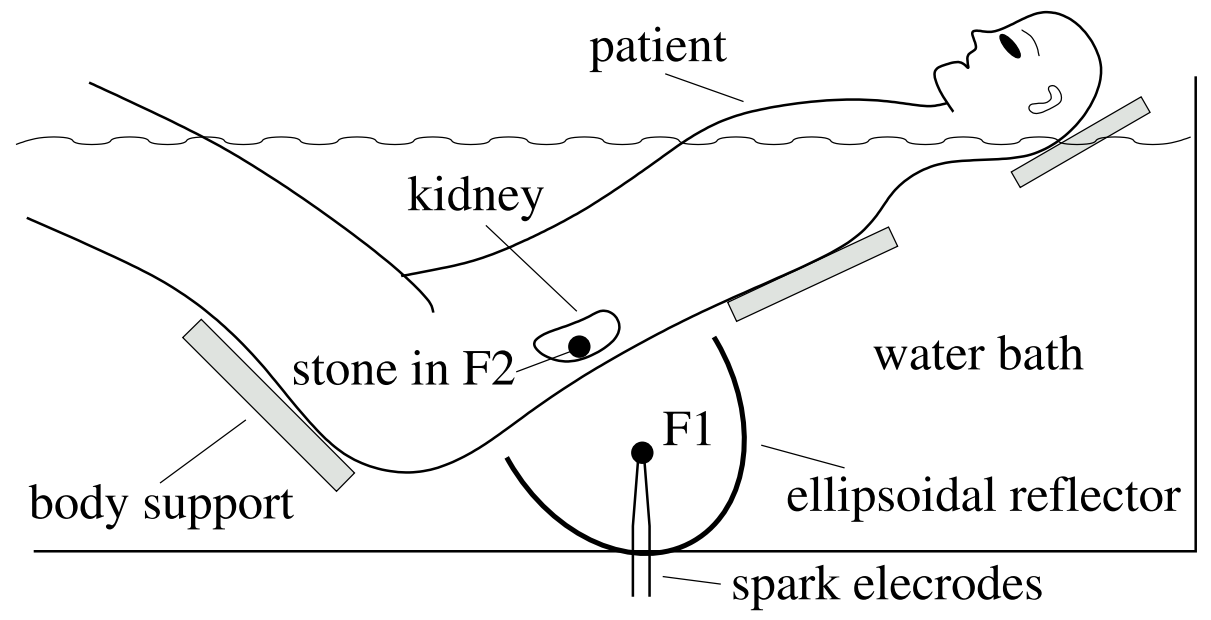

Figure 3.1: Schematic setup of an HM3 Dornier lithotripter for the disintegration of renal calculi. The shock wave is created by an underwater spark in the focal point F1 of an ellipsoidal reflector. It is refocused by the reflector on the second focal point F2. The patient is arranged in a way that F2 aligns with the kidney stone.

side the patient's body and are focused percutaneously on the stone to be disintegrated. After successful treatment the residual fragments are small enough to be voided in the urine or removed using transurethral endoscopy. Fragmentation efficiencies, anesthesia requirements, side effects, and the possibility of targeting different locations inside a patient depend on the setup of the lithotripter. Different companies use various approaches in shock wave creation and focusing. Figure 3.1 depicts the setup for an HM3 Dornier lithotripter, a widely used electrohydraulic system for the disintegration of renal calculi. Shock waves are created by an underwater spark discharge at a focal point F1 of an ellipsoidal reflector. By discharging storage capacitors at a high voltage $(\sim 20 \mathrm{kV})$ across the spark gap a plasma is generated. The liquid between the electrodes is rapidly vaporized and a spherical shock wave is emitted from the focal point F1. This shock wave is partially reflected and focused by the ellipsoidal reflector at the secondary focal point F2. With the help of x-ray or ultrasound imaging techniques the patient is arranged in a way that F2 is located at the position of the kidney stone. Other commercial lithotripters also use piezoelectric or electromagnetic methods for shock wave creation. The former method, for example implemented by Richard Wolf $\mathrm{GmbH}$, uses a fast high voltage pulse to excite a layer of piezoceramic elements. The single elements produce pressure waves, which are focused due to a spherical geometry of the layer shown in Fig. 3.2 (a). In electromagnetic lithotripters, for example produced by Siemens, a strong current is driven through a coil 
(a)

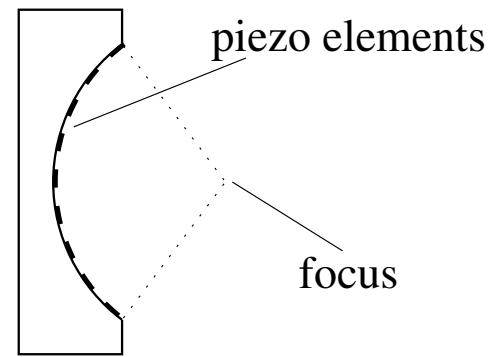

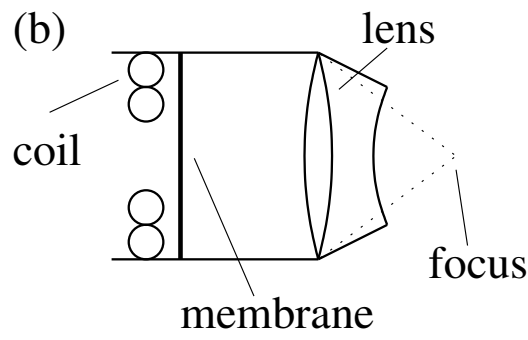

Figure 3.2: Schematic setup for a piezoelectric (a) and an electromagnetic (b) lithotripter.

opposite to a metal membrane. The varying magnetic field causes a current to flow in the membrane and the electromagnetic forces accelerate the membrane away from the coil creating a pressure pulse in the adjacent medium. The pressure wave is subsequently focused by an acoustic lens as shown in Fig. 3.2 (b). Generally, the maximum amplitude of a focused shock wave is in the order of $40 \mathrm{MPa}$ with rise times of less than 100 nanoseconds. The positive pressure pulse is followed by a diffraction induced negative tail of several microseconds, which is responsible for the generation of cavitation. Up today it is still under dispute, which are the predominant mechanisms for lithotripter induced stone fragmentation $[65,66]$. Possible mechanisms presently discussed include:

1. Spalling due to the generation of tensile stress caused by shock wave reflections at a pressure-release boundary $[65,66,67,68,69]$. Reflected tensile waves may be focused or superimposed with the initial tensile pulse of the lithotripter shock wave depending on the stone geometry $[70,71,68]$.

2. Damage caused by cavitation erosion $[72,65,66,67,68,69,73,74,75,76,77,78]$, which is presumably associated with jet impacts on the stone and secondary shock wave creation on a small scale during bubble collapse.

3. Tension induced by pressure gradients of a sharply focused shock wave [67].

4. Squeezing of the stone due to circumferential pressure [67].

It is likely that all mechanisms contribute to stone fragmentation in lithotripsy. Experiments indicate that some of the mechanisms mentioned above may work synergetically to achieve the desired small fragments after shock wave treatment [69]. 


\subsubsection{Theoretical description of shock waves}

For a theoretical understanding of shock waves, the compressibility of the medium has to be taken into account. The one-dimensional linear wave equation for a pressure wave $P$ with sound velocity $c$

$$
\frac{\partial^{2} P}{\partial x^{2}}=\frac{1}{c^{2}} \frac{\partial^{2} P}{\partial t^{2}}
$$

can be derived from the equations of mass and momentum conservation under the assumption of small particle velocities $u$ and small density changes $|\Delta \rho| \ll \rho_{0}$ of the medium. These assumptions lead to a constant propagation velocity $c$ of any part of the wave regardless of the local pressure or density. While these approximations are justified for small amplitude waves, they cannot be applied to the general analysis of shock waves.

The equations for the conservation of mass, momentum, and energy for a compressible fluid neglecting dissipation and viscous forces read

$$
\begin{gathered}
\frac{\partial \rho}{\partial t}+\nabla(\rho \vec{u})=0 \\
\rho \frac{\partial \vec{u}}{\partial t}+\rho(\vec{u} \nabla) \vec{u}=-\nabla P \\
\rho \frac{d E}{d t}=\frac{P}{\rho} \frac{d \rho}{d t},
\end{gathered}
$$

with the specific internal energy $E$ being the sum of thermal and chemical energy per unit mass. To complete the set of equations an equation of state is needed. The Tait equation yields a simple relation in weakly compressible media:

$$
\frac{P+B}{P_{0}+B}=\left(\frac{\rho}{\rho_{0}}\right)^{n} .
$$

$B$ and $n$ are parameters depending on the medium. For pressures up to $10 \mathrm{GPa}$ in water at $20^{\circ} \mathrm{C}$ we can use $B \approx 305 \mathrm{MPa}$ and $n \approx 7.15$ [79]. Note that equation (3.3) yields a direct relation between pressure and density $P=P(\rho)$ if $B$ and $n$ are taken as constants. Since dissipation was neglected changes of state are isentropic and the equation of energy (3.2) becomes superfluous for a pressure calculation. Another often used equation of state for weakly compressible fluids is the stiff gas equation

$$
P=(n-1) \rho E-n B \quad,
$$

which produces similar results to the Tait equation for small pressure values [80]. 


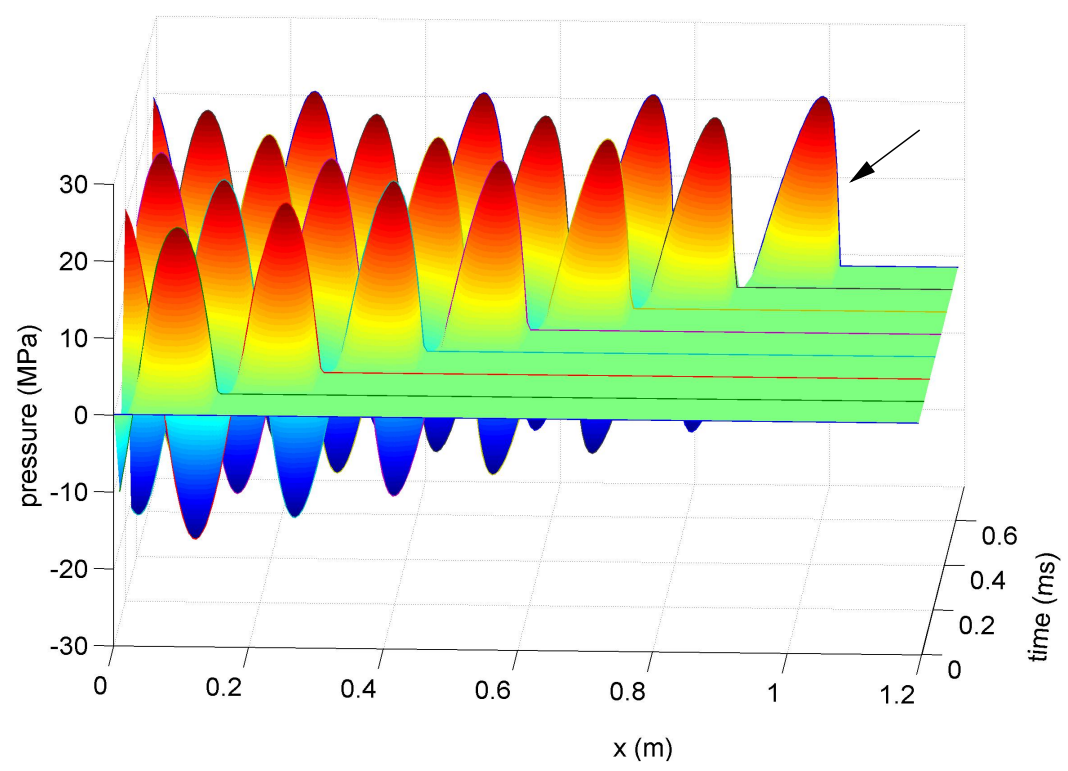

Figure 3.3: Steepening of a sinusoidal pressure wave in water due to nonlinear propagation for eight different times. The amplitude of the pressure is color coded, red and blue displaying high and low pressures, respectively. The arrow indicates the steep front developed during propagation.

As mentioned before, shock waves can arise due to nonlinear propagation of a pressure wave. Figure 3.3 shows the steepening of a high amplitude sinusoidal wave after propagation along the $\mathrm{x}$-axis at 8 different time steps. The results were obtained by solving the equations (3.1) and (3.3) in one dimension using a finite element method. Parameters have been chosen to simulate a pressure wave in water at $20^{\circ} \mathrm{C}$ with $\rho_{0}=998 \mathrm{~kg} / \mathrm{m}^{3}$ and $P_{0}=0.1 \mathrm{MPa}$. At $x=0$ a sinusoidal excitation pressure is applied as a boundary condition. The wave propagates from left to right and finally steepens so that the pressure front becomes almost discontinuous as indicated by the arrow in Fig. 3.3. The steepening occurs due to the increase of sound velocity with pressure. Thus parts of the wave with high pressure travel faster than regions with low pressure and the leading edge of each positive pressure pulse will become progressively steeper. At some point the leading edge will approach a discontinuity and dissipative factors have to be taken into account. However, in experiments it has been shown that shock waves may indeed travel with an almost discontinuous front and dissipation effects are very small [64]. Kirkwood approximated the thickness of a shock front in water taking into account the dissipated enthalpy. For a pressure of 20 kilobars the calculations predict a shock front of about $6 \mathrm{~nm}$ [64]. In this regime the approximation of a discontinuous front is justified and leads to a set of simple 
equations relating the speed of the shock front to the pressure. Let us denote the pressure, density, and normal particle velocity of the fluid immediately in front of the shock wave as $P_{0}, \rho_{0}$, and $u_{0}$ and behind the shock wave as $P, \rho$, and $u$. For an observer moving with the shock front of velocity $U$ the fluid will apparently move towards him with a velocity of $U-u_{0}$. The mass per unit area entering the front in time $d t$ will therefore be $\rho_{0}\left(U-u_{0}\right) d t$. From conservation of mass this must be equal to the mass per unit area leaving the front and we get:

$$
\rho_{0}\left(U-u_{0}\right)=\rho(U-u)
$$

Another equation can be derived from the balance of force. The difference in momentum per unit area and time for the fluid entering and leaving the shock front is given by:

$$
\rho_{0}\left(U-u_{0}\right) u_{0}-\rho(U-u) u=\rho_{0}\left(U-u_{0}\right)\left(u_{0}-u\right)
$$

Neglecting dissipation, the change in momentum must equal the pressure difference across the shock front, which yields:

$$
P_{0}-P=\rho_{0}\left(U-u_{0}\right)\left(u_{0}-u\right) \quad .
$$

Furthermore, we know that the change in energy must be compensated by the work done by the pressures $P$ and $P_{0}$. With $E_{0}$ and $E$ being the specific internal energy per unit mass before and after the shock wave we get:

$$
P u-P_{0} u_{0}=\rho_{0}\left(U-u_{0}\right)\left(E-E_{0}+\frac{1}{2}\left(u^{2}-u_{0}^{2}\right)\right) .
$$

Hereby, the difference in kinetic energy is expressed as

$$
\Delta E_{k i n}=\rho_{0}\left(U-u_{0}\right) d t \cdot \frac{1}{2}\left(u^{2}-u_{0}^{2}\right)
$$

Equations (3.4) and (3.5) were first developed by Rankine. The equation of energy balance (3.6) was later added by Hugoniot (see [64]). In the following chapters we will only regard shock waves which travel into a previously undisturbed medium. Therefore, we can set the initial velocity $u_{0}=0$ and receive a simpler form of equations (3.4-3.6):

$$
\begin{aligned}
\rho(U-u) & =\rho_{0} U \\
P-P_{0} & =\rho_{0} U u \\
E-E_{0} & =\frac{1}{2}\left(P+P_{0}\right)\left(\frac{1}{\rho_{0}}-\frac{1}{\rho}\right) .
\end{aligned}
$$


With the first and second equation of eqs. (3.7) we can express the shock velocity in terms of density and pressure:

$$
U=\sqrt{\left(\frac{\rho}{\rho_{0}}\right) \frac{P-P_{0}}{\rho-\rho_{0}}} .
$$

Together with the equation of state (3.3) the shock velocity is a function of pressure alone and we get:

$$
U=\sqrt{\frac{P-P_{0}}{\rho_{0}-\rho_{0}\left(\frac{P+B}{P_{0}+B}\right)^{-1 / n}}} .
$$

Similarly, we can express the particle velocity as a function of pressure:

$$
u=\frac{\rho-\rho_{0}}{\rho} \cdot U=\sqrt{\left(1-\left(\frac{P+B}{P_{0}+B}\right)^{-1 / n}\right) \frac{P-P_{0}}{\rho_{0}}} .
$$

In Fig. 3.4 the speed of the shock front and particle velocity of eqs (3.9) and (3.10) are plotted for pressures up to $4 \mathrm{GPa}$. The local speed of sound $c=\sqrt{d p / d \rho}$ can further be derived from the Tait equation (3.3)

$$
c=\sqrt{\frac{n \rho^{n-1}}{\rho_{0}^{n}}\left(P_{0}+B\right)}=\sqrt{\frac{n}{\rho_{0}}\left(\frac{P+B}{P_{0}+B}\right)^{\frac{n-1}{n}}\left(P_{0}+B\right)} .
$$

Upon reflection of a pressure wave at a low acoustic impedance boundary the pressure is inverted. Figures 3.5 and 3.6 depict such an event for a 1-dimensional pressure pulse incident from the left. The vertical and horizontal axes display time and space in the 2-dimensional plots, respectively. Amplitudes of the pressure (Fig. 3.5) and the particle velocity (Fig. 3.6) are color coded. At the right boundary the pressure was fixed to maintain a constant ambient pressure value of $100 \mathrm{kPa}$. This boundary condition characterizes a low acoustic impedance boundary, for instance a water-air interface. It can be seen in Fig. 3.5 that the pressure pulse is turned into a tensile pulse after reflection at the boundary. This effect is particularly important for the generation of cavitation, which can arise when the pressure of the tensile pulse drops below the vapor pressure. When shock waves are applied in a human body reflections at low acousitic impedance boundaries (e.g. the lungs) should therefore be minimized to avoid secondary bioeffects due to cavitation. However, cavitation may also arise during lithotripsy in the tensile pulse following the initial shock wave. Delius et al. $[81,82,78]$ have reported on tissue damage after shock wave application, which is probably attributed to cavitation generated in this way. 


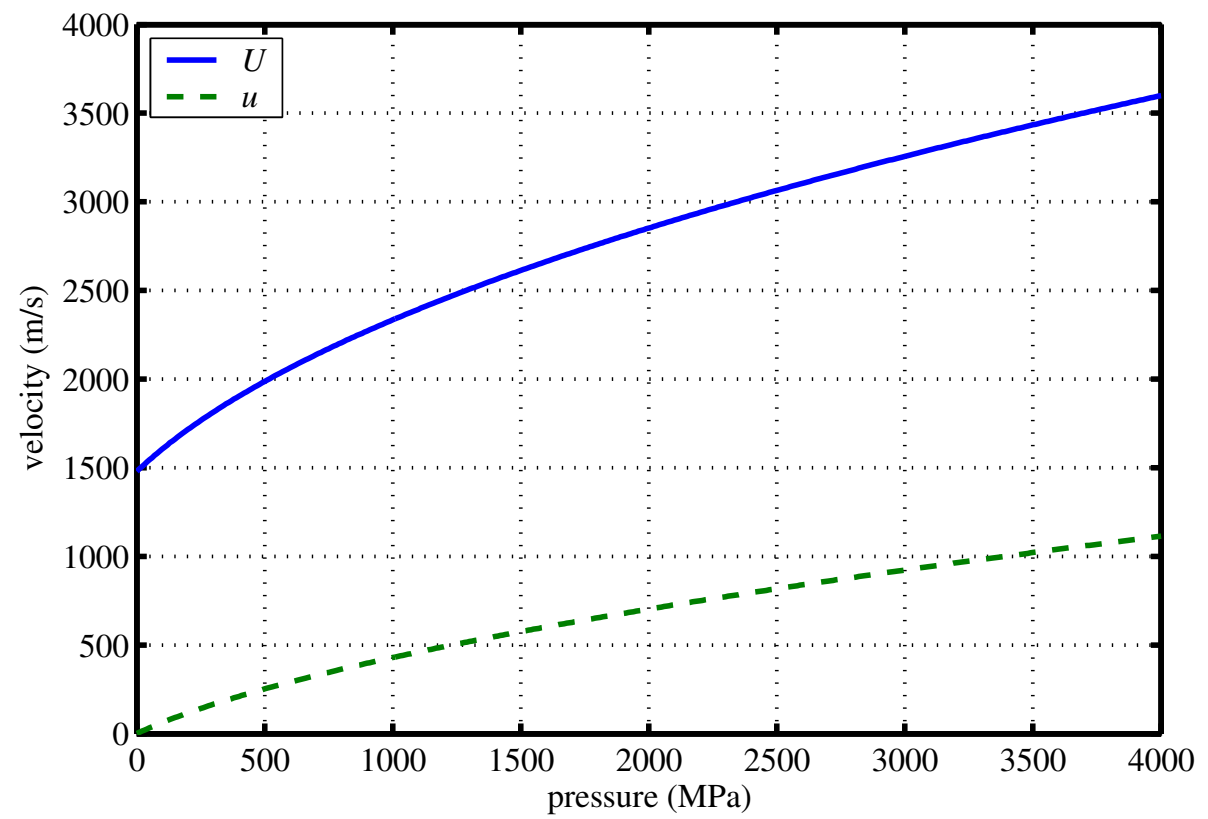

Figure 3.4: Speed of the shock front $U$ and of the particle velocity $u$ as a function of pressure as derived from eqs (3.9) and (3.10).

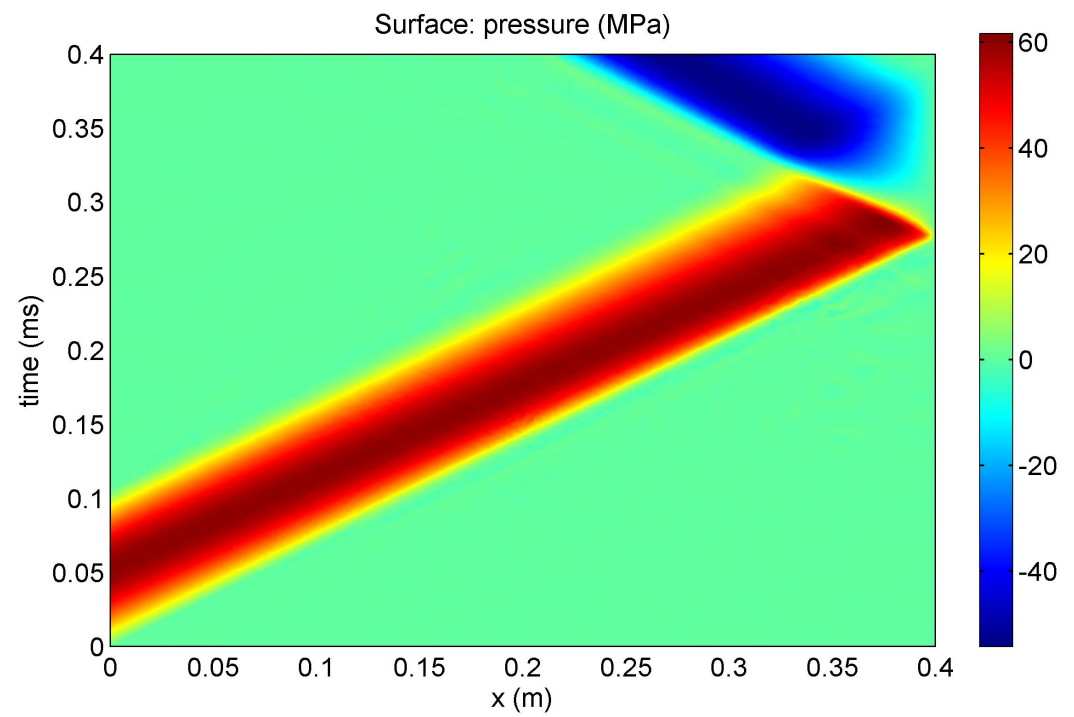

Figure 3.5: Reflection of a high amplitude pressure pulse at a low acoustic impedance boundary. Amplitudes of the pressure pulse are color coded, red and blue displaying high and low pressure values, respectively. 


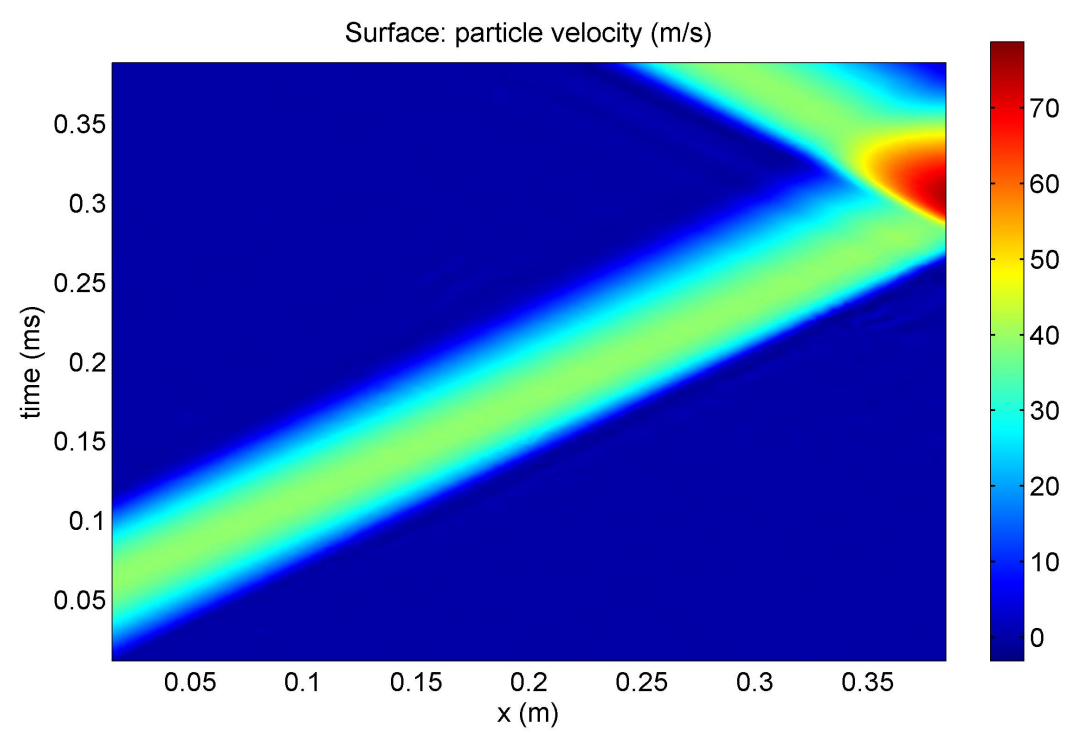

Figure 3.6: Particle velocity of the pressure pulse shown in Fig. 3.5. High particle velocities are colored red and low particle velocities are colored blue.

\subsection{Spherical bubble dynamics}

\subsubsection{Besant's model for an empty bubble}

A first approach to model the dynamics of an excited bubble has been proposed by Besant in 1859 [83]. He considered a spherical empty bubble in an incompressible liquid. With $\dot{R}$ as velocity of the bubble wall, the radial velocity $u$ of an incompressible liquid at position $r$ can be described as:

$$
u=\frac{R^{2} \dot{R}}{r^{2}} \quad, \quad R \leq r<\infty
$$

The velocity potential $\Phi$ of the liquid is obtained by integration over $u$ :

$$
\Phi=-\int_{r}^{\infty} u d r^{\prime}=-\frac{R^{2} \dot{R}}{r} .
$$

Using Bernoulli's Theorem we therefore get a relation between the radial velocity and the pressure $P_{l}$ in the liquid at position $r$ :

$$
\frac{P_{l}-P_{\infty}}{\rho}=-\frac{\partial \Phi}{\partial t}-\frac{1}{2} u^{2}=\frac{2 R \dot{R}^{2}+R^{2} \ddot{R}}{r}-\frac{1}{2} \frac{R^{4} \dot{R}^{2}}{r^{4}}
$$

Here, $P_{\infty}$ and $\rho$ are the pressure far away from the bubble and the density of water, respectively. Looking at a position next to the bubble boundary we have to substitute $R$ 
for $r$ in equation (3.14), which yields Besant's formula for an empty bubble:

$$
\frac{P_{l}-P_{\infty}}{\rho}=R \ddot{R}+\frac{3}{2} \dot{R}^{2}
$$

If we apply an external driving pressure $P_{e x}$ to the system, the remote pressure in the liquid can be written as $P_{\infty}=P_{0}+P_{e x}, P_{0}$ being the hydrostatic pressure.

\subsubsection{Rayleigh-Plesset model}

In an extension of Besant's model it is considered that a realistic bubble is not empty but filled with gas and vapor [84]. Let us first consider a bubble at equilibrium radius $R_{0}$ without external driving pressure. The pressure inside the bubble $P_{b u b, e q}$ is given by the partial pressures of vapor $P_{v}$ and gas $P_{g a s, e q}$ :

$$
P_{b u b, e q}=P_{g a s, e q}+P_{v}=P_{0}+\frac{2 \sigma}{R_{0}}
$$

Although we call $R_{0}$ the equilibrium radius, the bubble will eventually dissolve because of the excess pressure induced by the surface tension $\sigma$. If we consider now a quasi-static increase of pressure and assume the gas inside the bubble to follow a polytropic law, we can write for the gas pressure:

$$
P_{g a s}=P_{g a s, e q}\left(\frac{R_{0}}{R}\right)^{3 \gamma}=\left(P_{0}+\frac{2 \sigma}{R_{0}}-P_{v}\right)\left(\frac{R_{0}}{R}\right)^{3 \gamma}
$$

with $\gamma$ being the polytropic exponent. Assuming the vapor pressure to remain constant, we get for the pressure inside the bubble:

$$
P_{b u b}=\left(P_{0}+\frac{2 \sigma}{R_{0}}-P_{v}\right)\left(\frac{R_{0}}{R}\right)^{3 \gamma}+P_{v} .
$$

The pressure in the liquid directly at the bubble boundary $P_{l}$ equals the pressure inside the bubble without the term contributed by the surface tension:

$$
P_{l}=\left(P_{0}+\frac{2 \sigma}{R_{0}}-P_{v}\right)\left(\frac{R_{0}}{R}\right)^{3 \gamma}+P_{v}-\frac{2 \sigma}{R}
$$

Using this relation in equation (3.15) yields:

$$
R \ddot{R}+\frac{3}{2} \dot{R}^{2}=\frac{1}{\rho}\left[\left(P_{0}+\frac{2 \sigma}{R_{0}}-P_{v}\right)\left(\frac{R_{0}}{R}\right)^{3 \gamma}+P_{v}-\frac{2 \sigma}{R}-P_{\infty}\right]
$$


Equation (3.20) was derived in 1950 by Noltingk and Neppiras [85,86]. In 1952 it was extended by Poritsky [87] to incorporate the effects of the viscosity $\mu$ of water leading to an energy dissipation term at the bubble surface:

$$
R \ddot{R}+\frac{3}{2} \dot{R}^{2}=\frac{1}{\rho}\left[\left(P_{0}+\frac{2 \sigma}{R_{0}}-P_{v}\right)\left(\frac{R_{0}}{R}\right)^{3 \gamma}+P_{v}-\frac{2 \sigma}{R}-\frac{4 \mu \dot{R}}{R}-P_{\infty}\right] .
$$

Equation (3.21) is commonly called Rayleigh-Plesset or RPNNP (Rayleigh-PlessetNoltingk-Neppiras-Poritsky) equation.

So far the liquid is assumed to be incompressible. Keller and Miksis [88] derived a model to describe the dynamics of an acoustically excited bubble in a slightly compressible fluid. By including a small compressibility of the liquid the effects of acoustic radiation are taken into account. This is especially important for large amplitude oscillations. The consideration of acoustic radiation leads to smaller maximum amplitudes of the strongly forced bubble.

In a slight modification of the model given by Parlitz et al. [89] the equation reads:

$$
\left(1-\frac{\dot{R}}{c}\right) R \ddot{R}+\frac{3}{2} \dot{R}^{2}\left(1-\frac{\dot{R}}{3 c}\right)=\left(1+\frac{\dot{R}}{c}\right) \frac{P_{l}}{\rho}+\frac{R}{\rho c} \frac{d P_{l}}{d t}
$$

with the pressure in the liquid being:

$$
P_{l}(R, \dot{R}, t)=\left(P_{0}-P_{v}+\frac{2 \sigma}{R_{0}}\right)\left(\frac{R_{0}}{R}\right)^{3 \gamma}-\frac{2 \sigma}{R}-4 \mu \frac{\dot{R}}{R}-P_{0}+P_{v}-P_{e x} \sin (2 \pi f t)
$$

The excitation pressure in this equation is given by a sinusoidal function with the driving frequency $f$. Equation (3.22) has been used in the following chapters to model the dynamics of observed pressure-excited bubbles. The sinusoidal driving term $P_{e x} \sin (\omega t)$ has therefore been replaced by $P_{e x}(t)$ to allow for arbitrary pressure functions fitted from pressure measurements in the experiments. It should be noted however, that the equation was derived under the assumption that the bubble size is small compared to the wavelength of the driving pressure. Further assumptions are:

- The bubble remains spherical during its oscillations.

- Buoyancy and other translational forces are small enough to be neglected.

- The gas inside the bubble follows an ideal polytropic law. 
- Pressure and temperature are homogeneous inside the bubble.

- There is no gas diffusion or heat transfer across the bubble boundary.

- The vapor pressure inside the bubble remains constant.

- The change of density and the compressibility of the liquid are small.

- The speed of sound in the liquid remains constant.

- No chemical reactions take place inside the bubble .

During the last 50 years, the Rayleigh-Plesset model has been extended in terms of several aspects. This includes primarily the compressibility of the liquid $[90,88]$, generation of shock waves [91, 92,93], transition from an isothermal to an adiabatic collapse, diffusion [94], chemical reactions [95,96], evaporation, and condensation [96].

All models yield relatively good results for the slow expansion phase and the beginning of the spherical collapse. However, due to the small bubble size and high bubble wall velocities it is difficult to confirm the models during the late stage of bubble collapse. Although high-speed imaging techniques have improved greatly during the last ten years, it is still not possible to capture the final collapse of a highly spherical bubble.

\subsection{Aspherical bubbles}

Most oscillating bubbles occurring in technical applications are not really spherical during their later contraction phase. Already small disturbances in the radial symmetry of an expanded bubble will lead to large deformations during collapse [12]. A breaking of radial symmetry can be caused, for example, by a boundary in the vicinity of the bubble. The action of strongly collapsing cavitation bubbles near boundaries is known to lead to material damage and erosion. Damage caused by hydrodynamic cavitation occurs for example at marine propellers, turbine blades, or in high pressure pumps. Acoustic cavitation involving aspherical bubble dynamics $[11,12,18]$ is held responsible for ultrasonic cleaning and erosion. To elucidate the mechanisms of erosion bubble collapse in the vicinity of rigid or soft, mostly plane boundaries has been studied extensively in experiments. Therein, bubbles are created and excited by different methods, which include optical breakdown by focused laser light $[14,97,98,16,99,100,101,102,103]$, electric spark generation [104,105], micro explosions [106], and application of lithotripter shock waves [17,107]. 
A solid wall imposes a restriction on the fluid flow. Upon collapse, the bubble side facing the boundary is therefore slower and subjected to a smaller pressure than its opposite. Depending on the pressure excitation and the initial distance to the boundary, the bubble involutes at the side opposite to the boundary and develops a liquid jet directed towards the boundary. When the jet hits the other side of the bubble a shock wave is emitted and the bubble wall is pushed towards the boundary. A second shock wave is emitted, when the jet strikes the boundary. The dynamics is strongly influenced by the standoff parameter $\gamma$, which is defined by the ratio of the distance between bubble center and boundary and the initial bubble radius $\left(\gamma=s / R_{0}\right)$. For bubbles with $100 \mu \mathrm{m}$ radius at a pressure excitation of $10 \mathrm{MPa}$ and a standoff parameter of $\gamma=1.1$ the pressure at the wall below the jet has been estimated to reach $1400 \mathrm{MPa}$ [80]. With the jet penetrating the center of the bubble, the bubble develops a toroidal shape. Such a scenario is shown in Fig. 3.7 for a spark generated bubble collapsing close to a glass surface. The pressure at the boundary propagates upwards into the jet. Upon reflection at the phase border surrounding the jet, the pressure pulse is converted to a tensile pulse, which may cause additional cavitation. This phenomenon is called counter-jet since it travels in the opposite direction of the initial jet away from the boundary [97,108]. During rebound the bubble usually fragments into a cloud of smaller bubbles, which can be seen in frame 5 and 6 of Fig. 3.7. Upon collapse, these small bubbles may give rise to further damage at the boundary. Cavitation arising in the human body for example in the rarefaction phase of lithotripter generated shock wave or during ultrasonic imaging with contrast agent bubbles will probably also be aspherical due to restrictions of flow by surrounding tissue or blood vessels. For liquid-liquid boundaries and soft material boundaries it has been shown that the jet can also be directed away instead of towards the boundary depending on the standoff parameter $\gamma[109,110,111]$.

Due to the highly nonlinear perturbations during aspherical bubble collapse it is difficult to describe the dynamics in a quantitative model. However, some groups have successfully used boundary integral as well as finite element methods to model numerically certain aspects of aspherical bubble dynamics like jet formation and toroidal rebound [112,110, $113,114,115,80$. 


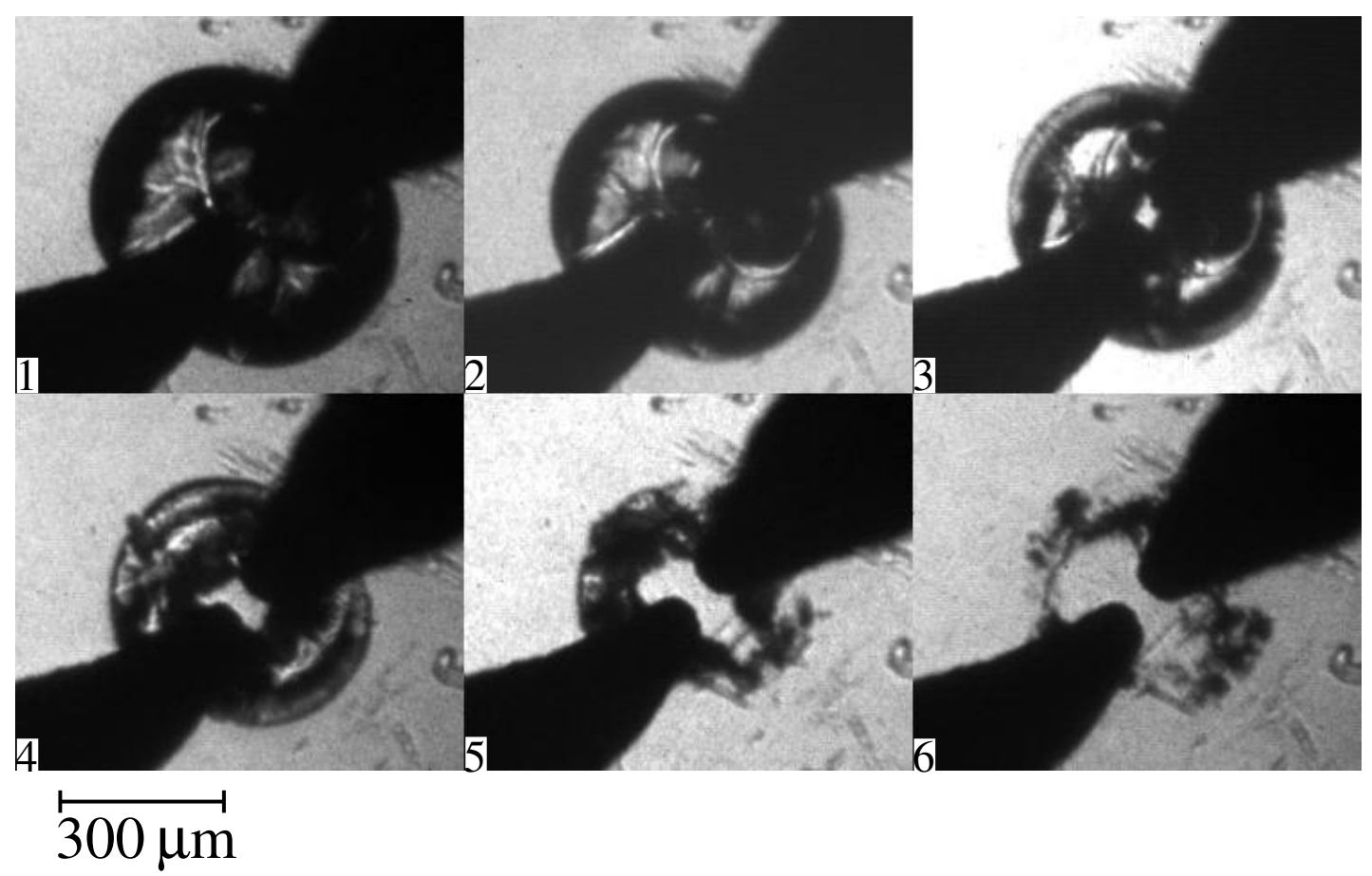

Figure 3.7: A bubble is generated by plasma formation after voltage discharge between two steel electrodes. It collapses aspherically onto a glass surface. The first frame starts approximately $90 \mu$ s after spark ignition and the bubble is already in contraction phase. Exposure and interframe times are $100 \mathrm{~ns}$ and $2.1 \mu \mathrm{s}$, respectively. 


\section{Chapter 4}

\section{Shock wave induced interaction of microbubbles and boundaries}

In most of the experiments performed to study aspherical bubble collapse, single, fairly large bubbles of maximum radius $R_{\max } \geq 200 \mu \mathrm{m}$ have been generated in a well-defined geometry, allowing for long distance microscopy. Due to the limited magnification it is more difficult to investigate smaller bubbles using such a setup. However, small cavitation bubbles may play an important role in phenomena like lithotripsy $[73,75,65]$, shock wave cleaning methods [116], optoporation [117], and surface activation in sonochemistry [8]. Their dynamics cannot be simply deduced from observations of larger bubbles without verification because of possible stronger surface tension effects. Furthermore, in the applications above, bubbles tend to interact with neighboring bubbles or solid particles, for example, with the stone during lithotripsy or the debris from the ultrasonic cleaning process. The purpose of the present study is to investigate bubble collapse of small bubbles in a heterogeneous environment, i.e. under conditions that are close to real-world applications, featuring multiple, irregularly placed solid boundaries. Salt grains in water have turned out to provide a suitable medium as seed bubbles are released during the slow $\mathrm{NaCl}$ dissolution.

In the following experiments bubbles are excited by an acoustic shock wave and their dynamics is recorded by high-speed video microscopy, making accessible details on small spatial and temporal scales. In particular, interactions of single and multiple bubbles (initial radii between 5 and $150 \mu \mathrm{m}$ ) with salt crystals are investigated by observation of bubble dynamics and particle destruction [118]. 


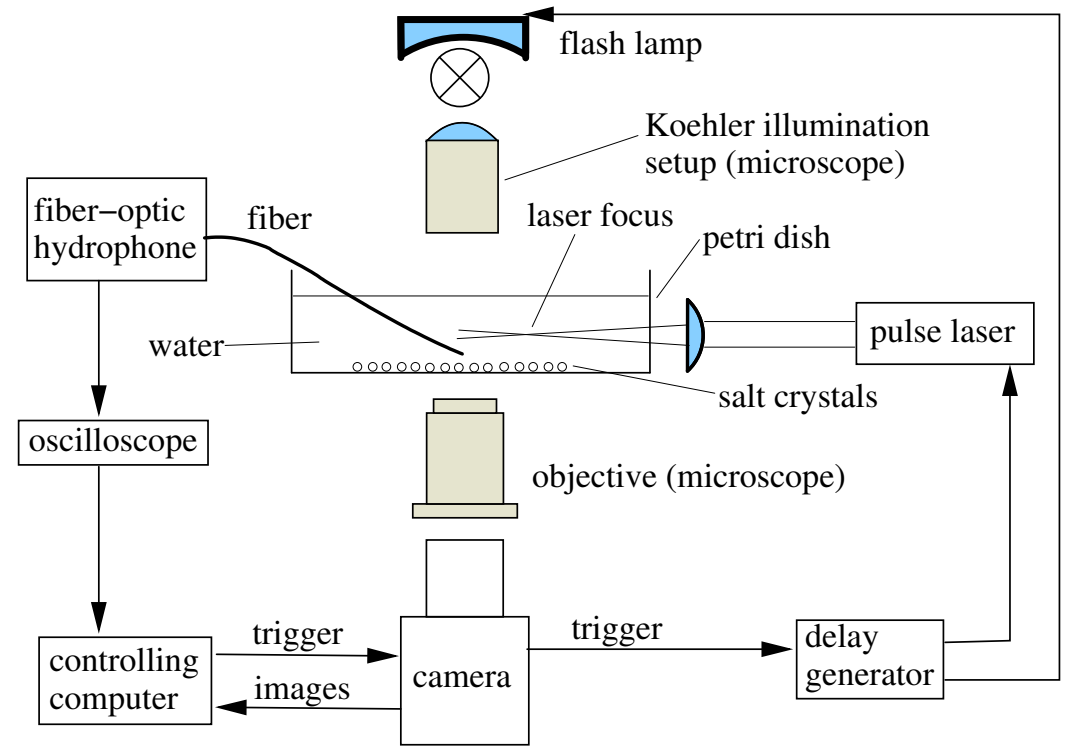

Figure 4.1: General setup for creation and observation of shock waves and subsequent bubble dynamics.

\subsection{Methods}

The experimental setup is depicted in Fig. 4.1, its main component being a table top microscope connected to a high-speed camera. Grains of salt are placed on the plane surface of a duran petri dish filled with water. During the slow dissolution of the salt crystals differently sized bubbles are observed that probably originate from air pockets contained in inclusions or in surface clefts of the grains. The largest bubbles reach diameters of over $200 \mu \mathrm{m}$ and could originate from gas trapped in a crystal at higher pressure. The bubbles are excited with a laser induced shock wave and their dynamics is captured with a high-speed camera.

\subsubsection{Shock wave creation and measurement}

A Q-switched Nd:YAG laser with a maximum pulse energy of $780 \mathrm{~mJ}$ was used to deliver pulses of $\approx 8 \mathrm{~ns}$ duration at a wavelength of $\lambda=1064 \mathrm{~nm}$. The light was focused with a single lens into the petri dish containing the salt grains. For laser plasma generation where a highly spherical focus is required usually more than one lens is used to minimize aberration effects [119]. In this setup aberration minimization was not necessary, because the laser generated plasma was only needed to create the shock wave. The op- 
tical breakdown in water by nanosecond pulses is primarily caused by the absorption of light at impurities in the liquid. This process leads to thermal ionization of molecules. The initially released free electrons are called lucky electrons. They take up more energy from the laser irradiation and ionize further molecules upon achieving enough momentum. Thereby an avalanche ionization process is initiated leading to an exponential growth of free carriers during the laser pulse [120]. For shorter laser pulses at high intensities the direct ionization of water molecules by multiphoton absorption can also contribute to the plasma generation.

Inside the plasma temperatures ${ }^{1}$ and pressures are very high. The surrounding water is accelerated outwards and a shock wave is created. Measurements have shown that the maximum amplitude of such a shock can exceed $100 \mathrm{kbar}$ at the plasma edge [122]. After recombination of ions and electrons a bubble remains, which is filled with water vapor and its dissociation products. An example of a laser created bubble is given in Fig. 4.2. Its dynamics can be described fairly well with the spherical bubble models given in Section 3.2 .

The pressure of the shock waves was recorded with a fiber-optic hydrophone FOPH 300 developed by Eisenmenger and Staudenraus at the University of Stuttgart [123]. The underlying principle for the measurements is based on the change of refractive index of the liquid with pressure. The light of a laser diode with a wavelength of $\lambda=810 \mathrm{~nm}$ is coupled into a glass fiber. Some of the light is reflected at the fiber tip due to the difference of refraction indices between water and the fiber core. The reflected light is detected by a photodetector, which is coupled into the fiber by an optical fiber coupler and converted to an electrical signal. When a pressure wave passes the fiber, a change of refractive index is induced. Positive pressures raise the index of refraction of water thereby lowering the difference to the index of the fiber core. As a result, less light is reflected and we receive a lower signal at the photodetector. The electrical signal given by the photodetector in response to a pressure wave can be deconvoluted with the impulse response of the system and calibrated to yield pressure values. The spatial resolution of the optical hydrophone depends on the orientation of the fiber tip and is limited by the size of the fiber core, being $140 \mu \mathrm{m}$ in diameter. Rise times are electronically limited to about 10 ns. Benefits of the fiber optical hydrophone are the improved spatial and temporal resolutions compared to conventional hydrophones based on piezoelectric techniques. A drawback however, is the low sensitivity to pressure changes, which is limited to a few

\footnotetext{
${ }^{1}$ The temperature of a plasma created with a $5 \mathrm{~ns}$ Nd:YAG laser pulse at an energy of $4 \mathrm{~mJ}$ has been measured to be approximately $10000 \mathrm{~K}$ [121]
} 


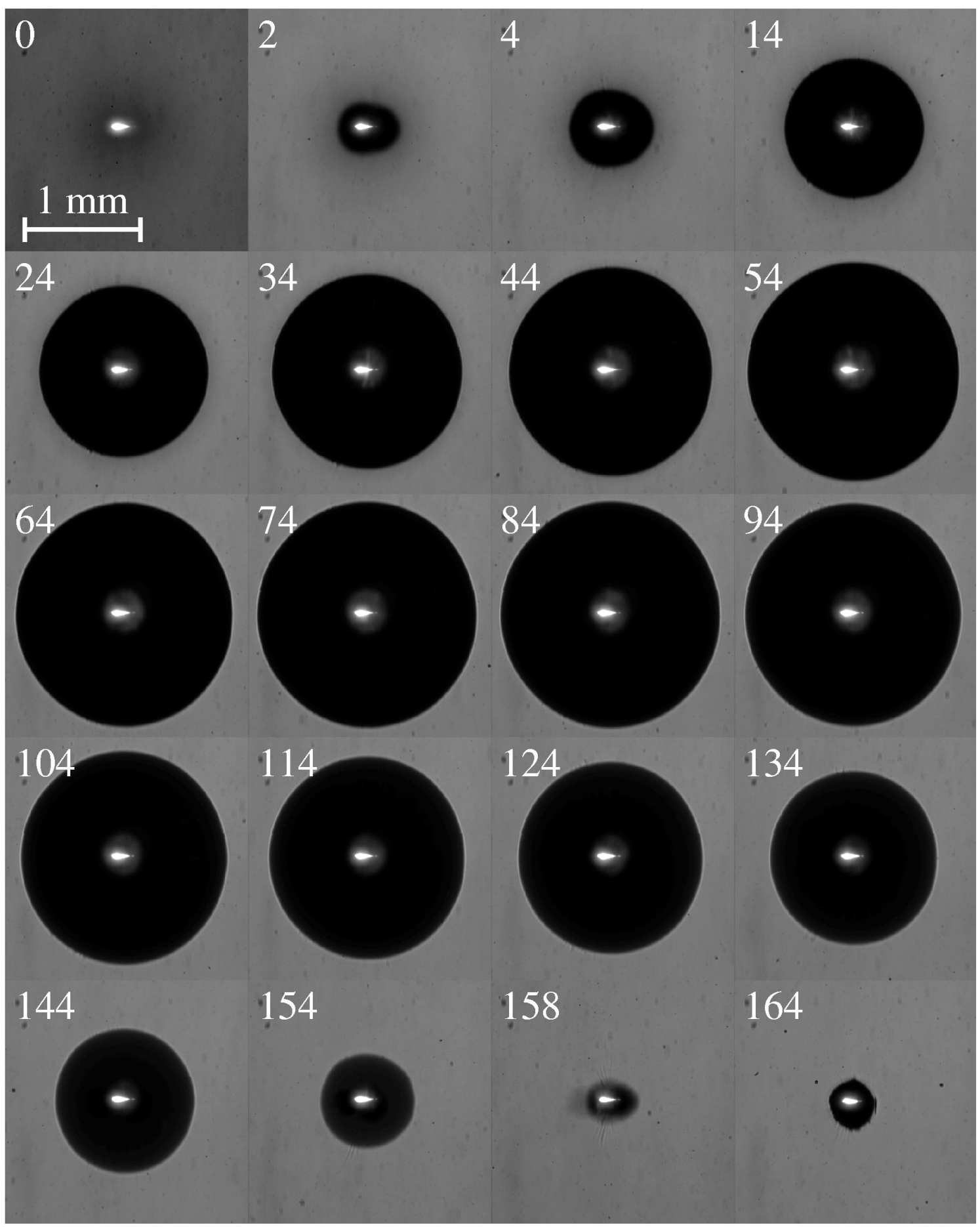

Figure 4.2: Laser generated bubble at ambient pressure of 1.3 bar. The numbers given in each frame refer to the time after optical breakdown in microseconds. 
bars. For reproducible pressure pulses a better signal to noise ratio can be achieved by averaging multiple events.

In the setup of Fig 4.1 the fiber optical hydrophone was read out by a digital oscilloscope (TDA784A, Tektronix). During pressure measurements the laser generated plasma was located on the optical axis of the fiber with a distance of approximately $3 \mathrm{~mm}$ to the fiber tip. After each shot, the memory of the oscilloscope was transferred to the controlling computer and stored in a database for subsequent analysis. Twenty shots were recorded and averaged to reduce errors due to background noise. Fig. 4.3 (a) shows a typical recording of the hydrophone signal. The first positive signal is generated by the light of the breakdown plasma, which is collected by the optical fiber. The following large negative pulse represents the shock wave caused by the rapid plasma expansion (negative voltage values indicate positive pressure). This signal has been averaged, inverted, deconvoluted and calibrated [124] to display the excess pressure of the shock wave as depicted in Fig. 4.3 (b). A second positive pressure pulse appears approximately $270 \mu \mathrm{s}$ after recording of the plasma signal. It is caused by the shock wave created in the collapse of the laser generated bubble. However, its amplitude is significantly smaller than that of the initial pressure pulse. It is probably attenuated due to the bubble collapse being aspherical.

\subsubsection{High-speed microscopy}

The dish was mounted on an inverted Microscope (Axiovert 135 TV, Zeiss). A number of objectives with magnification ratios of $5 \times$ to $32 \times$ and numerical apertures of 0.1 to 0.4 were used to achieve different resolution when detecting shock waves or single $\mathrm{NaCl}$ grains. For the recording of shock waves only $10 \times$ magnification was used, which yielded a good compromise between resolution and low smear due to progression of the shock wave during exposure time. Images were captured with a high-speed camera (Imacon 468, DRS Hadland LTD). The camera possesses a pyramid beam splitter to divide the available light onto eight individual intensified charge coupled device sensors (ICCD sensors). Delay, gain, and exposure time of the ICCDs can be set independently with a controlling computer via a fiber optic link. The minimum exposure time of each channel is $10 \mathrm{~ns}$. This is also the minimum time delay between the start of two consecutive frames, giving rise to a maximum frame rate of 100 million pictures per second. Images of each sequence are stored internally before being transferred to the controlling computer.

Depending on the exposure time of the camera the dish was illuminated either with 


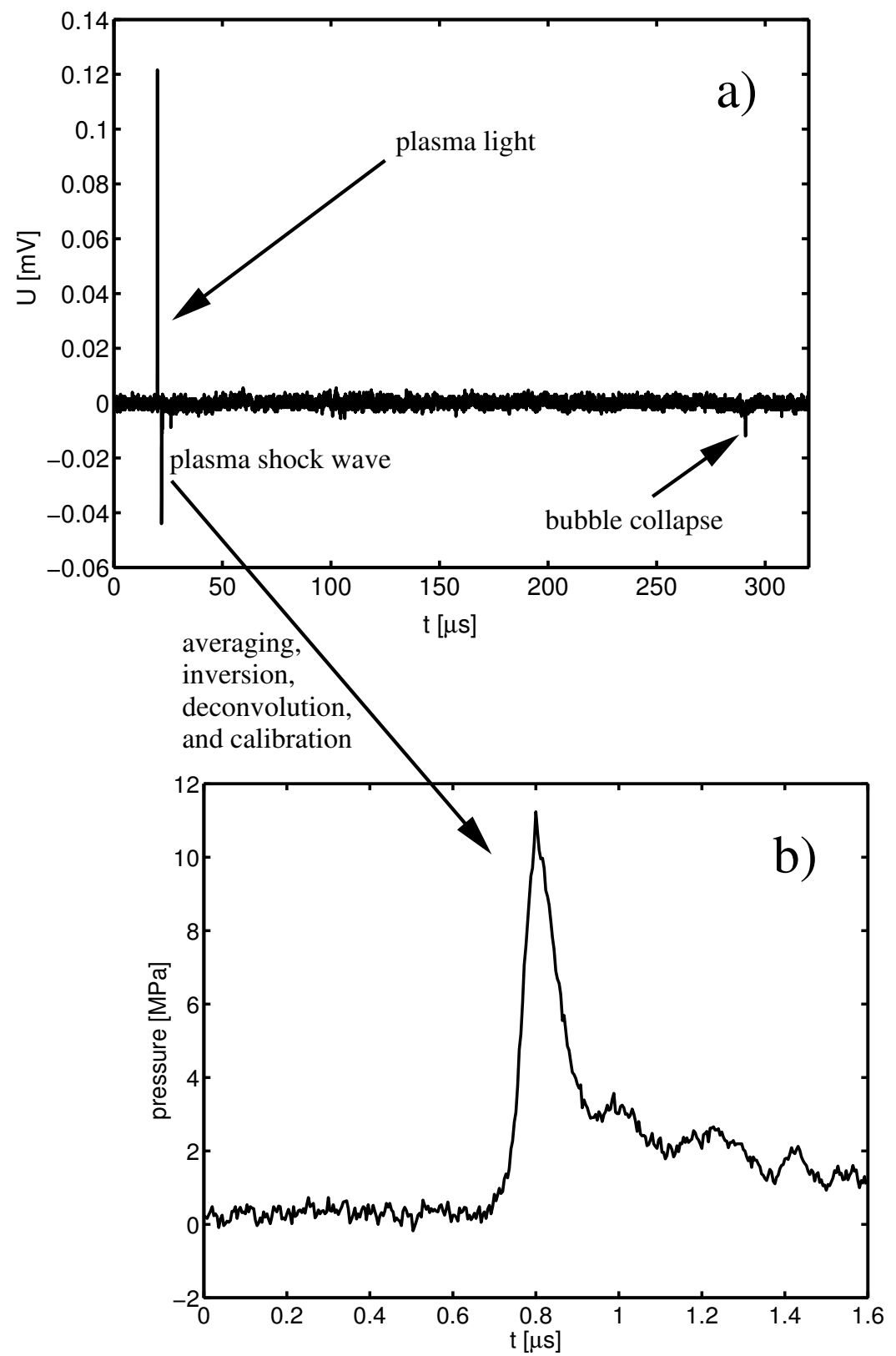

Figure 4.3: Hydrophone recording of a laser induced shock wave. (a): voltage signal, (b): pressure amplitude of primary shock wave with different time axis. 
a continuously emitting microscope lamp or with a xenon flash lamp coupled into the Koehler illumination of the microscope. The xenon flash lamp delivers a pulse of 500 Joule with $50 \mu \mathrm{s}$ (FWHM) duration. Timing of camera, flash lamp, and laser was synchronized by a delay generator (DG535, Stanford Research Systems). The trigger was initiated by the camera.

\subsubsection{Numerical modeling of the bubble dynamics}

To compare the observed radius time curves of the bubble dynamics with the spherical model derived in Section 3.2.2 the equations (3.22) and (3.23) have been rescaled and solved with an explicit Runge-Kutta method implemented by Matlab's ode45 routine. The rescaled equation reads:

$$
\begin{aligned}
\ddot{x} \frac{u_{0}}{t_{0}}\left(\left(1-\frac{u_{0} \dot{x}}{c}\right) r_{0} x+\frac{4 \mu}{\rho c}\right)= & -\frac{3}{2} \dot{x}^{2} u_{0}^{2}\left(1-\frac{\dot{x} u_{0}}{3 c}\right)-\frac{2 \sigma}{r_{0} \rho x}-\frac{4 \mu \dot{x} u_{0}}{\rho x r_{0}} \\
& +\frac{1}{\rho}\left(1+\frac{u_{0} \dot{x}}{c}\right)\left(-P_{0}+P_{v}-P_{e x}(\tau)\right) \\
& +\left(1+(1-3 \gamma) \frac{u_{0} \dot{x}}{c}\right) \frac{1}{\rho}\left(P_{0}-P_{v}+\frac{2 \sigma}{r_{0}}\right)\left(\frac{1}{x}\right)^{3 \gamma} \\
& -\frac{r_{0} x}{\rho c t_{0}} \dot{P}_{e x}(\tau),
\end{aligned}
$$

where $x=R / r_{0}, \tau=t / t_{0}$, and $\dot{x}=\dot{R} / u_{0}$ are the rescaled bubble radius, time and radial velocity with the corresponding scaling factors $r_{0}$ (initial bubble radius), $t_{0}=1 \mu \mathrm{s}$, and $u_{0}=r_{0} / t_{0}$. Hydrostatic pressure, sound velocity, density, surface tension, and viscosity of the liquid have been set to $P_{0}=100 \mathrm{kPa}, c=1500 \mathrm{~m} / \mathrm{s}, \rho=998 \mathrm{~kg} / \mathrm{m}^{3}, \sigma=$ $72.7 \mathrm{mN} / \mathrm{m}$, and $\mu=1 \mathrm{mPa} \cdot \mathrm{s}$, respectively. A polytropic exponent of $\gamma=1$ has been used in the calculations, assuming an isothermal process. The exciting pressure $P_{e x}$ has been approximated by fitting three Gaussian functions to the data recorded by the optical hydrophone using a nonlinear least squares method.

\subsection{Bubble-boundary and bubble-bubble interaction}

In Fig. 4.4 we can see the shock wave generated by the plasma expansion passing through the observed area. The peak amplitude was measured to be $11.2 \pm 2.8 \mathrm{MPa}$. Five hundred nanoseconds after shock wave passage neither the bubble indicated by the arrow in Fig. 4.4 


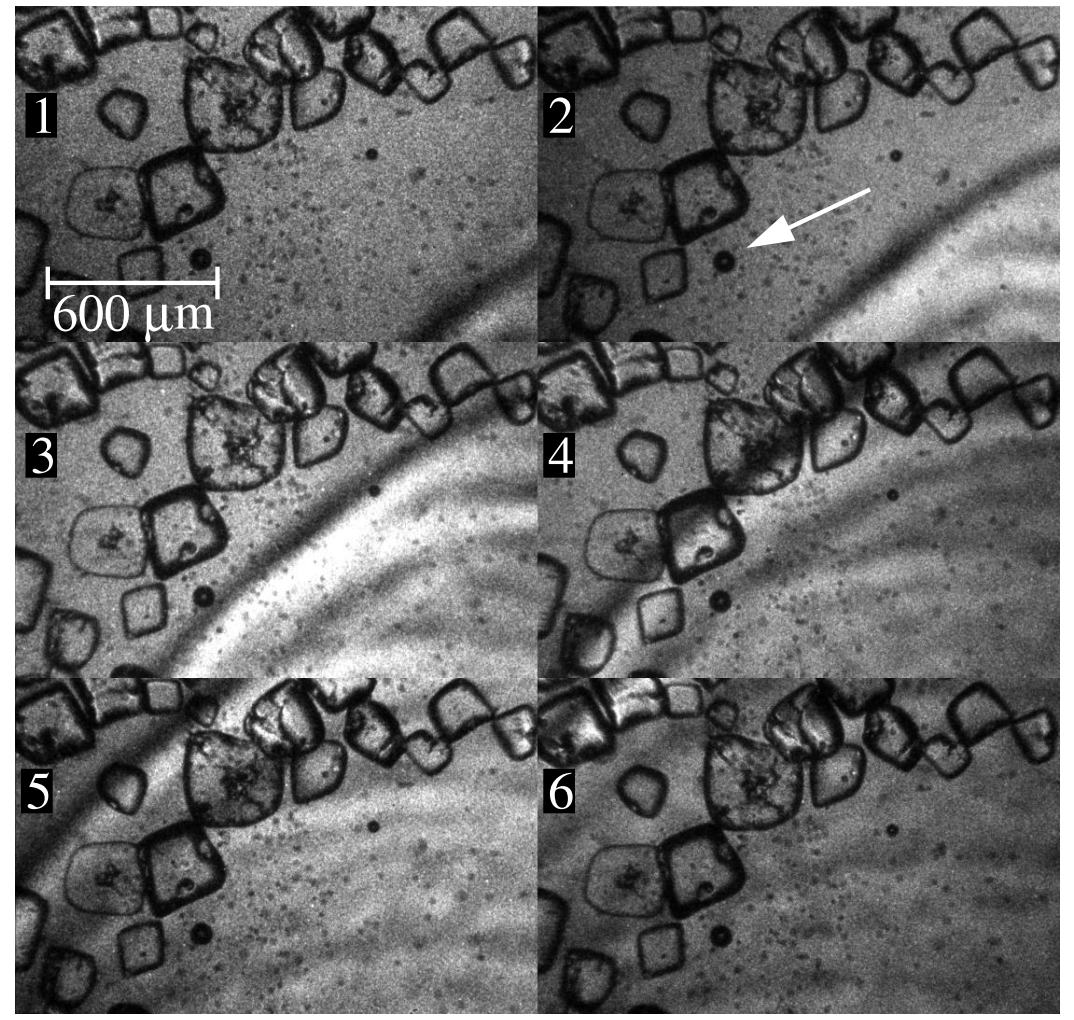

Figure 4.4: High-speed recording of a shock wave passing salt crystals and bubbles. Exposure and interframe times are $40 \mathrm{~ns}$ and $240 \mathrm{~ns}$, respectively. The arrow in frame 2 indicates a bubble position. A scale is given in frame 1. 


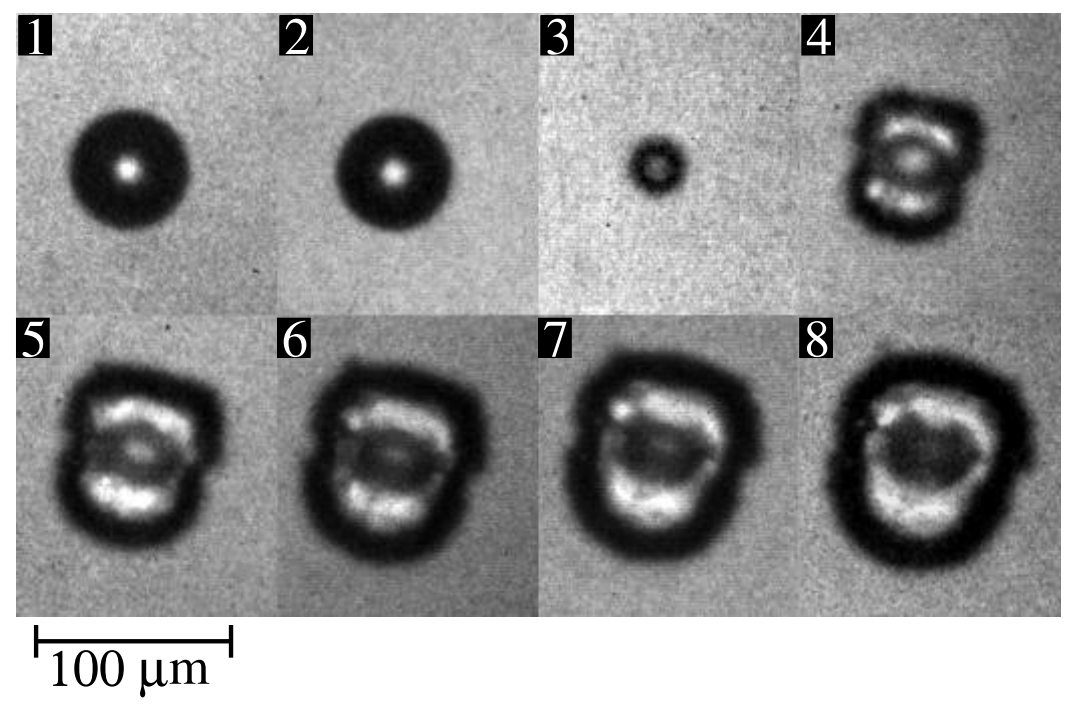

Figure 4.5: Collapse and torus-like rebound of a single bubble close to the rigid boundary of the petri-dish after shock wave application. The shock wave is incident from below. Frame 1 starts $\approx 0.3 \mu$ s before shock wave passage. Exposure and interframe times are $100 \mathrm{~ns}$ and $400 \mathrm{~ns}$, respectively.

nor the salt crystals display any reaction to the pressure excitation. Visible excitation of bubbles observed in these experiments starts to appear between $200 \mathrm{~ns}$ and $1 \mu \mathrm{s}$ after shock wave passage depending on bubble size and resolution. There is no detectable crack formation inside the salt grains due to mechanical stress by the initial shock wave.

Let us first consider the dynamics of small bubbles with only one rigid boundary nearby, i.e. the glass bottom of the petri dish. Similar to experiments with larger laser created bubbles close to rigid boundaries [16,101,103], the bubbles form a jet toward the boundary and exhibit a torus-like form after collapse (Fig. 4.5). The hole first seen in frame four appears at the position where the jet has penetrated the center of the bubble. The asymmetry of the torus arises probably due to the propagation direction of the shock wave, which hits the bubble from the bottom of the picture.

Figure 4.6 shows the experimental radius-time data of the bubble of Fig. 4.5 as seen through the petri-dish compared to the bubble dynamics predicted by the simple KellerMiksis model for spherical bubbles as given in equation (4.1). We can see from the figure that the qualitative bubble dynamics is similar for the experimental and calculated radius time curve. However, the spherical model predicts a smaller bubble radius upon rebound than is observed in Fig. 4.5. This is to be expected, since we observe an oblate ellipsoidal or toroidal instead of a spherical bubble because of the vicinity to the petri dish. The flow 


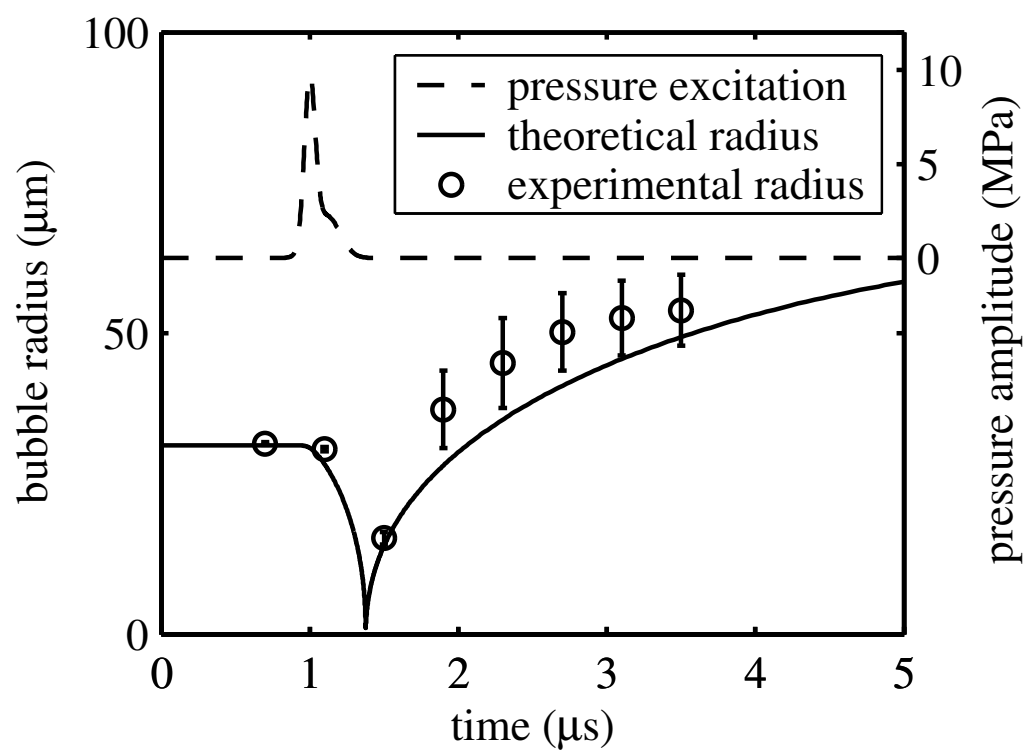

Figure 4.6: Comparison of the experimental radius time curve derived from Fig. 4.5 with the bubble dynamics calculated from the spherical model of equation (4.1). The dashed curve shows the excitation pressure used for the calculation.

field induced by the aspherical collapse forces the bubble to spread along the boundary. Looking through the petri dish we therefore overestimate the bubble volume by assuming a spherical geometry with the observed radius. For a toroidal form of the bubble after rebound with the slice diameter being equal to the ring diameter we obtain an enlargement of the observed radius by a factor of 1.2 under the assumption of equal volumes of torus and sphere. Furthermore, the model probably overestimates the damping of rebound oscillations by acoustic emission because of supposition of a spherical collapse.

After rebound the bubbles may exhibit different shapes. Figure 4.7 shows the rebound of a single bubble close to the rigid boundary of the petri dish with $7 \mu$ s interframe time. A channel arises from the center of the bubble and stays intact for several microseconds. It is directed at the petri dish and can be attributed to the jet. Since the bubble is already in rebound phase, the dark pin-like structure in the center might be caused by a counterjet. Counter-jets have been observed in side-view for large laser created bubbles near rigid boundaries [108] and are composed of small microbubbles. They probably emerge in the wake of a toroidal shock wave generated during a ring-like bubble collapse. We note that, unlike the larger laser induced bubbles investigated, the surface of the bubble in Fig. 4.7 remains fairly smooth during rebound even for direct boundary contact, corresponding 


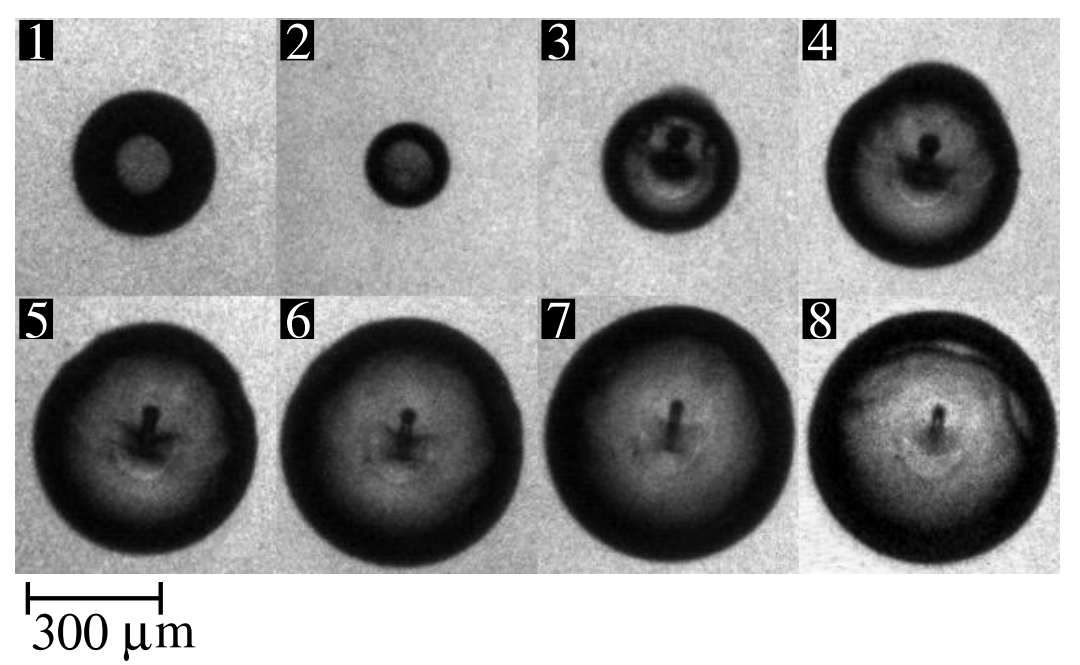

Figure 4.7: Collapse and rebound of a bubble after shock wave application. The shock wave is incident from below. Frame 1 starts $\approx 0.8 \mu$ s before shock wave passage. Exposure and interframe times are $1 \mu \mathrm{s}$ and $7 \mu \mathrm{s}$, respectively.

to a standoff parameter $\gamma=1$. This is probably caused by a greater influence of surface tension for smaller bubbles.

In Fig. 4.8 a similar situation is depicted. The interframe time is now $6 \mu$ s and the bubble size and shock wave amplitude are comparable to Fig. 4.7. However, in this case the surface of the bubble becomes unstable. Pinch-offs which partly separate and form small bubbles occur at the bubble's surface. The relevant parameters for surface instabilities upon rebound cannot be assessed from the present data. They probably depend on bubble size and details of the pressure train and aspherical collapse. Similar instabilities have been observed by Lauterborn during the rebound of laser created bubbles exhibiting inclined jets $[125]$.

Figure 4.9 shows the experimental radius-time curves of the bubbles of Figs. 4.7 and 4.8 and those predicted by the spherical model. Due to the long interframe time of the images it is possible to compare the radius time curves after their maximum expansion. As in Fig. 4.6, the observed bubble sizes are larger than predicted by the model. Note also that the time until second collapse is different. The model predicts a shorter collapse time after the first rebound than is observed in the experiment. One can assume that the increased collapse time is caused by the drag of flow imposed by the rigid boundary. During the initial collapse and expansion phase, the bubble is forced to spread along the boundary. At maximum expansion there is a large contact zone between bubble and boundary layer. As 


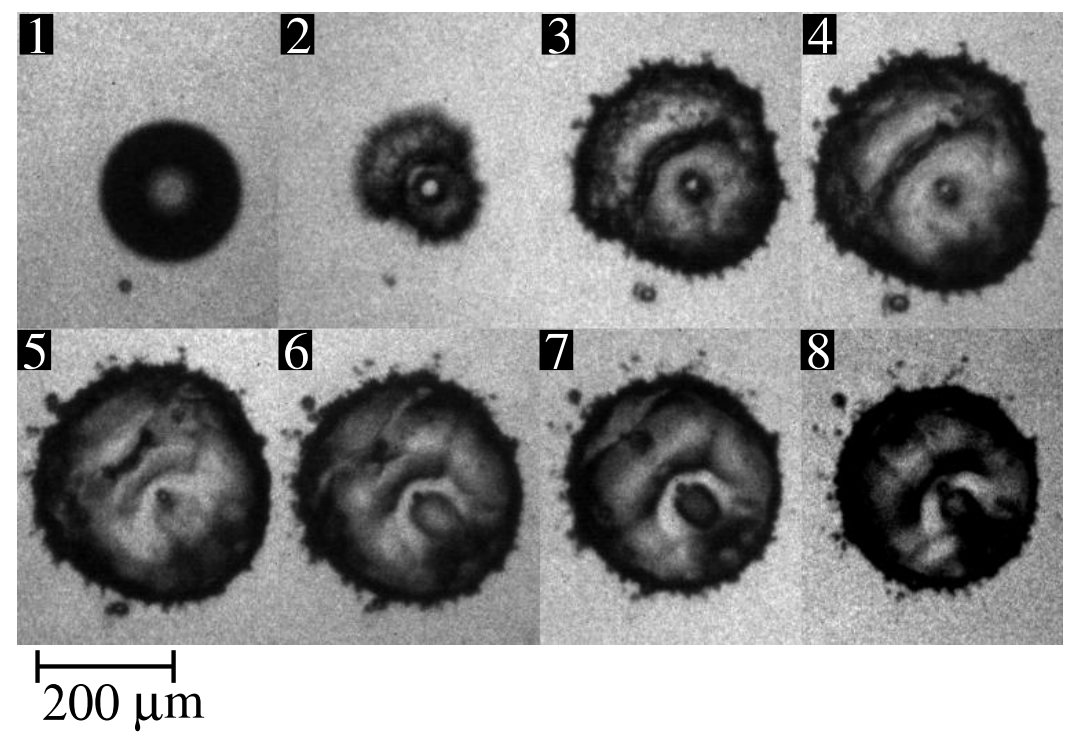

Figure 4.8: Collapse and rebound of a bubble after shock wave application. The bubble exhibits surface instabilities upon rebound. The shock wave is incident from the lower right. Frame 1 starts $\approx 0.8 \mu$ s before shock wave passage. Exposure and interframe times are $1 \mu \mathrm{s}$ and $6 \mu \mathrm{s}$, respectively.

the bubble starts to contract again, the flow is impeded at the boundary layer resulting in a shear stress on the surface of the petri dish. Similarly, a prolongation of collapse time for spark and laser generated bubbles near a solid wall has been observed [104,98]. Calculations by Strasberg also show a decrease in pulsation frequency of nonspherical gas bubbles in liquids [126]. For a better description of the experimental observations, a boundary integral $[112,113,114]$ or finite volume method [115] should be used to simulate aspherical bubble dynamics.

The collapse of bubbles and their subsequent dynamics is strongly influenced by the number of boundaries and other bubbles nearby. The preceding figures illustrate mainly a one-boundary influenced collapse of small bubbles resulting in a toroidal shape. However we have already seen a slight break of symmetry on the torus possibly due to the propagation direction of the shock wave in Fig. 4.5 and Fig. 4.8. The effect of the shock wave is more pronounced in Fig. 4.10. In this case the setup was slightly different than described above. Pressure waves were generated by focusing the laser on a copper target at a distance of approximately $2 \mathrm{~mm}$ to the bubble. Instead of dissolving salt crystals, the bubble was generated by electrolysis using an electrode with a diameter of $30 \mu \mathrm{m}$. The bubble is filled with hydrogen, because it originates from the negative electrode. In 


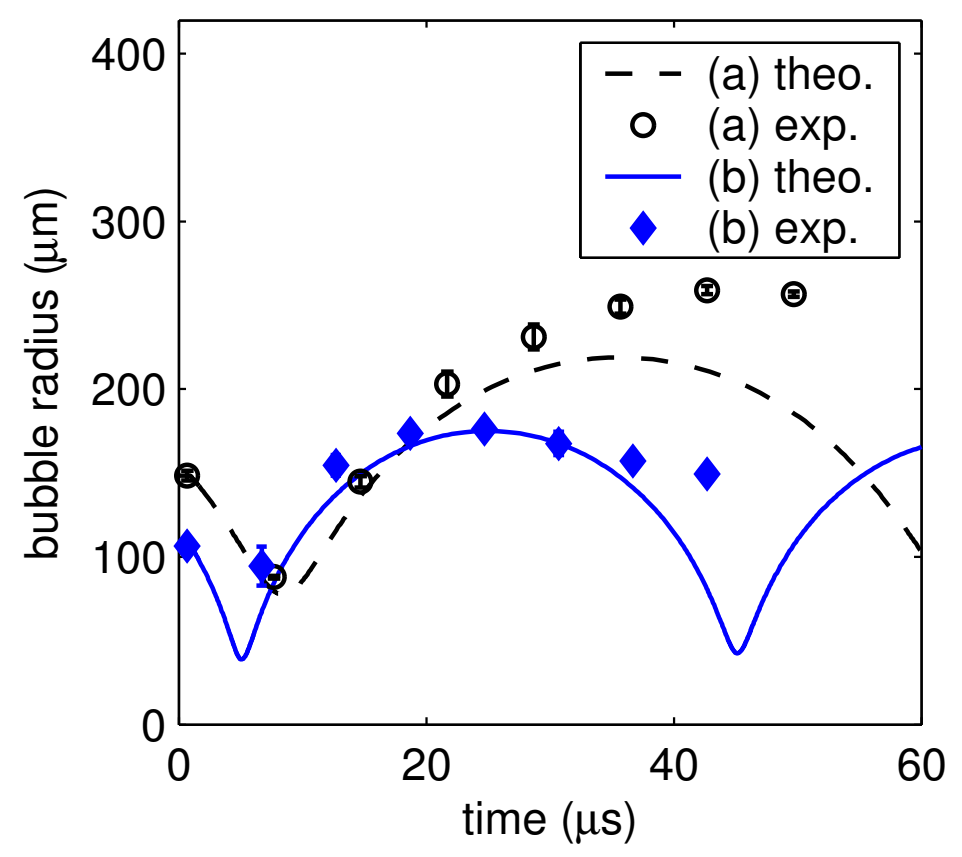

Figure 4.9: Comparison of the experimental radius time curves derived from Figs. 4.7 and 4.8 with the bubble dynamics predicted by the spherical model of equation (4.1). 


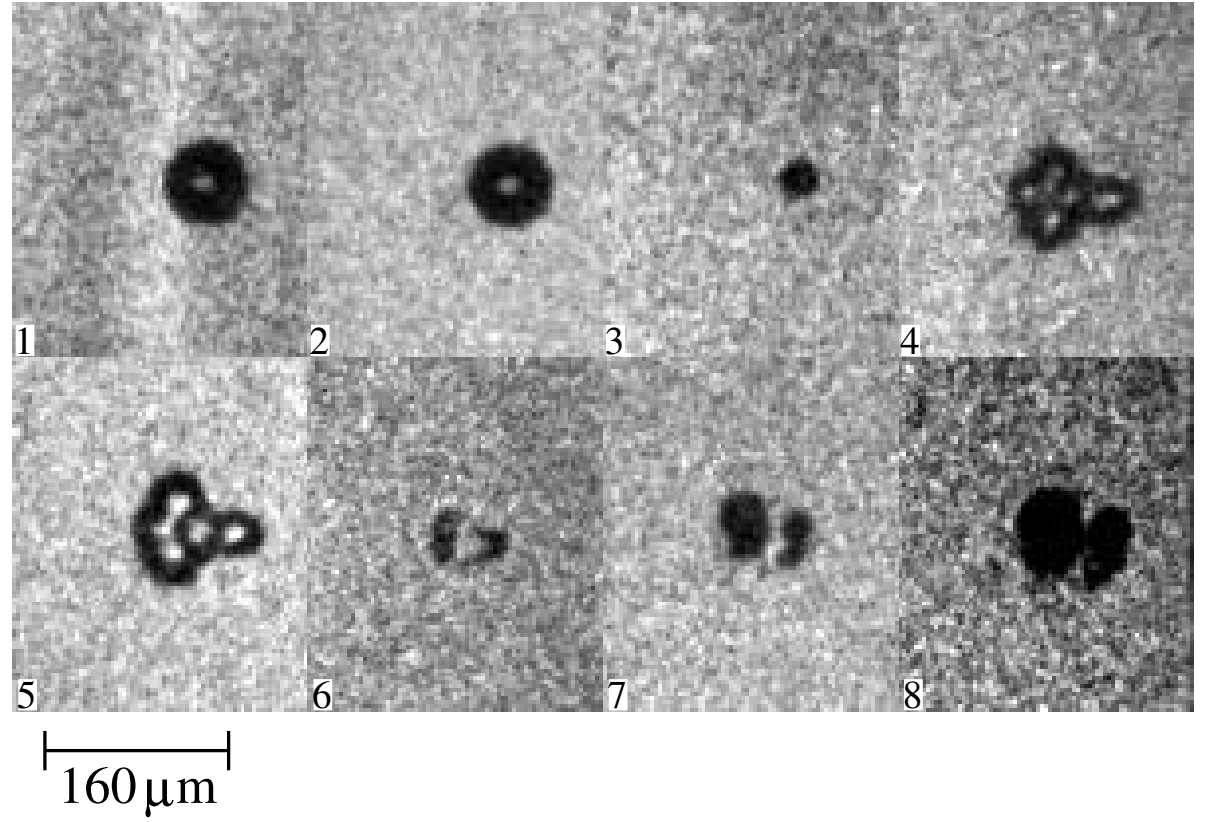

Figure 4.10: Bubble collapse and rebound due to pressure wave excitation. The pressure wave is incident from the right. Exposure and interframe times are $20 \mathrm{~ns}$ and $520 \mathrm{~ns}$, respectively.

the first frame the bubble is seen together with the pressure wave traveling from right to left. The bubble collapses approximately $1 \mu \mathrm{s}$ later and rebounds in a bird-foot shaped form in frame 4 and 5 . The propagation direction of the pressure pulse determines the orientation of the "bird-foot". The first digit is facing towards the pressure source and the other three digits are facing in the propagation direction of the shock wave. The bird would therefore be moving in the same direction as the pressure wave. Although the exact shape may vary for different bubbles the bird foot shape seems to be characteristic for a bubble collapse close to a rigid boundary under the influence of pressure wave propagation direction. In the center we can see the same circular structure found in toroidal bubbles, which is most probably caused by the jet penetrating the bubble. In frame 6 of Fig. 4.10 the bubble separates into two parts, the larger fraction facing in the propagation direction of the pressure wave.

If more than one bubble is present the rebound shapes of the bubbles remain the same. However, the orientation of the bird-foot is then determined by the propagation direction of the pressure pulse and also the position of neighboring bubbles. This is depicted in Fig. 4.11, where four bubbles created by electrolysis are excited by pressure pulses traveling from right to left. The general orientation of the bird-foot structure is governed by the 


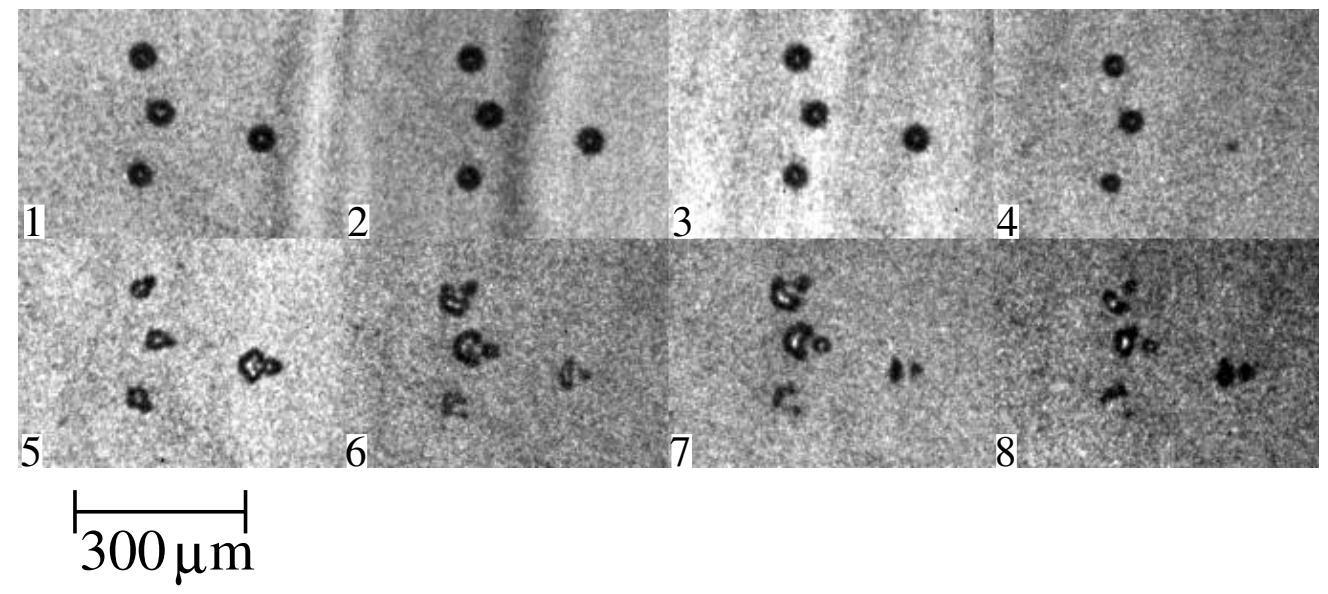

Figure 4.11: Bubble collapse and rebound due to pressure wave excitation. The pressure wave is incident from the right. Exposure and interframe times are $20 \mathrm{~ns}$ and $320 \mathrm{~ns}$, respectively.

propagation direction of the pressure wave. However, one can see that the bubbles at the top and at the bottom are influenced by the location of their neighbor. Therefore the birdfoot is oriented slightly towards the center bubble. The orientation towards neighboring bubbles increases with decreasing bubble distance.

Figure 4.12 shows seven small bubbles generated again by dissolution of salt crystals in an area of less than $200 \mu \mathrm{m}$ diameter. The shock wave is incident from below but we can also see that the collapse and rebound orientation is largely governed by adjacent bubbles. It is also likely that the direction of the jet is perturbed by neighboring bubbles. As seen in the two preceding figures, the bubbles partly split in the collapse phase. During rebound and oscillation phase, they reunite and approach each other.

On longer time scales this behavior leads to the accumulation of bubbles into clusters as can be seen in Fig. 4.13. The interframe time in this image is $9 \mu \mathrm{s}$. After shock wave application, the bubbles oscillate and touch their neighbors during rebound. Merging and translation towards each other due to the secondary Bjerknes force lead to a cluster in the horizontal plane of the petri dish.

The situation of bubble collapse changes when there is a second rigid boundary besides the petri dish. Attraction of an oscillating bubble to the boundaries can be seen in Fig. 4.14. The interframe time between the first six images is $500 \mathrm{~ns}$ to capture the initial collapse of the bubble. The next two interframe times are $20.1 \mu \mathrm{s}$ each. During that time, the bubble oscillates and splits into multiple fragments, which move toward the 


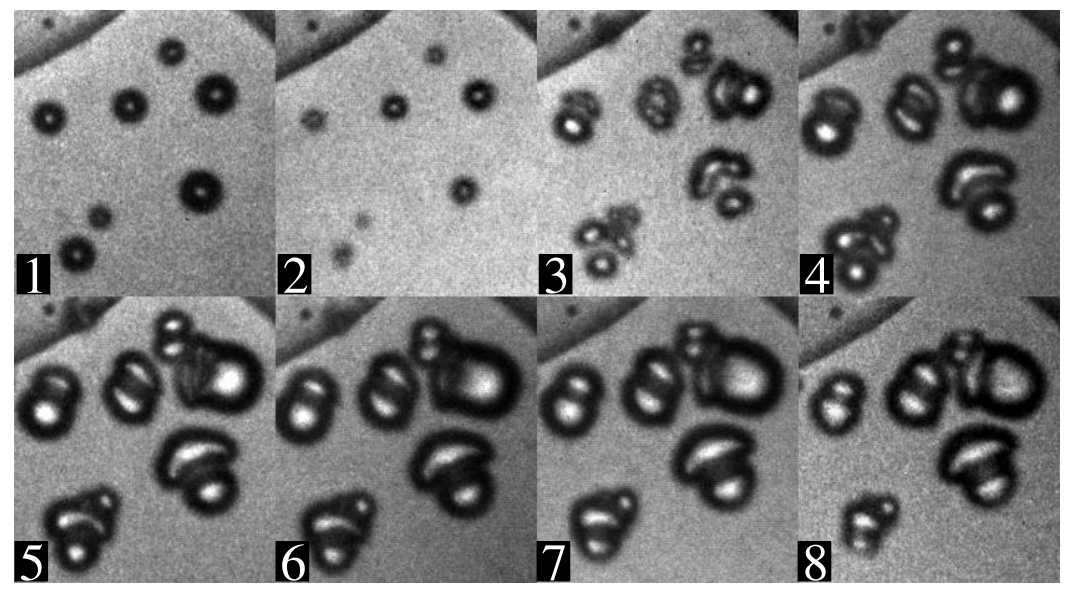

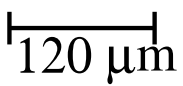

Figure 4.12: Collapse and rebound of multiple bubbles. The shock wave is incident from below. Frame 1 starts $\approx 0.3 \mu$ s before shock wave passage. Exposure and interframe times are $100 \mathrm{~ns}$ and $500 \mathrm{~ns}$, respectively.

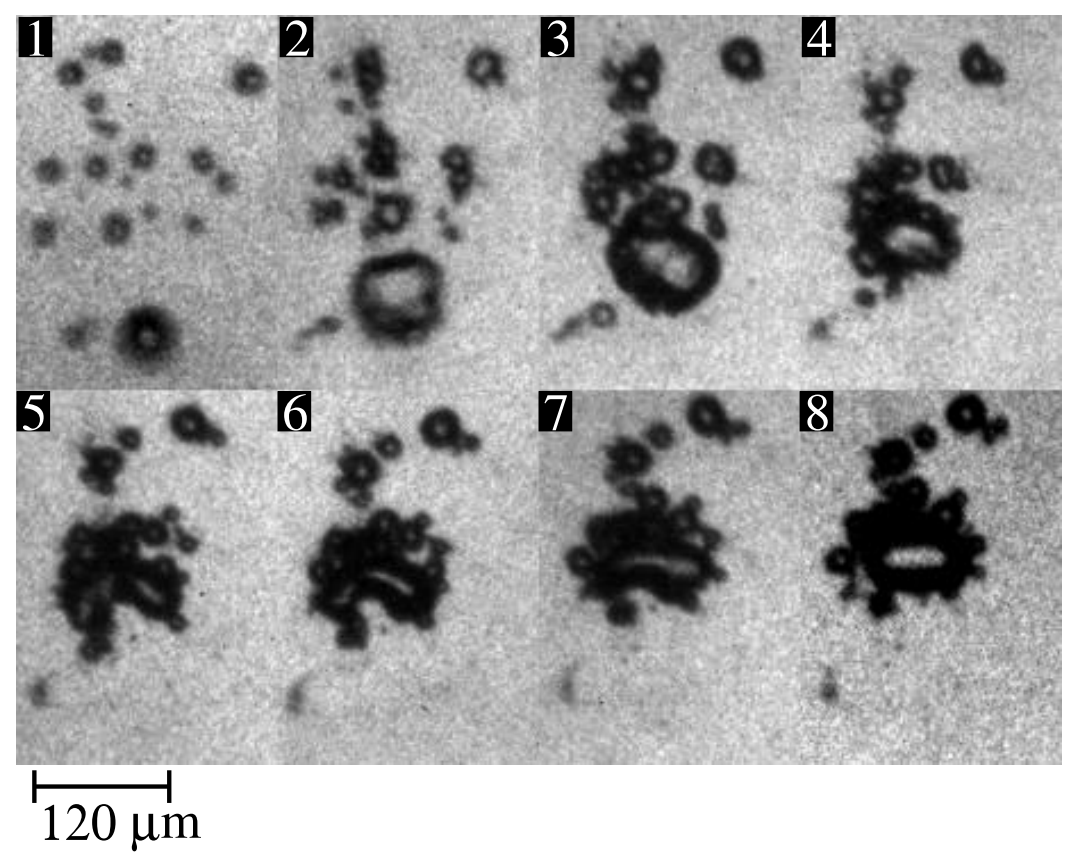

Figure 4.13: Clustering of shock wave excited bubbles. The shock wave is incident from the lower right. Frame 1 starts $\approx 0.9 \mu$ s before shock wave passage. Exposure and interframe times are $1 \mu \mathrm{s}$ and $10 \mu \mathrm{s}$, respectively. 


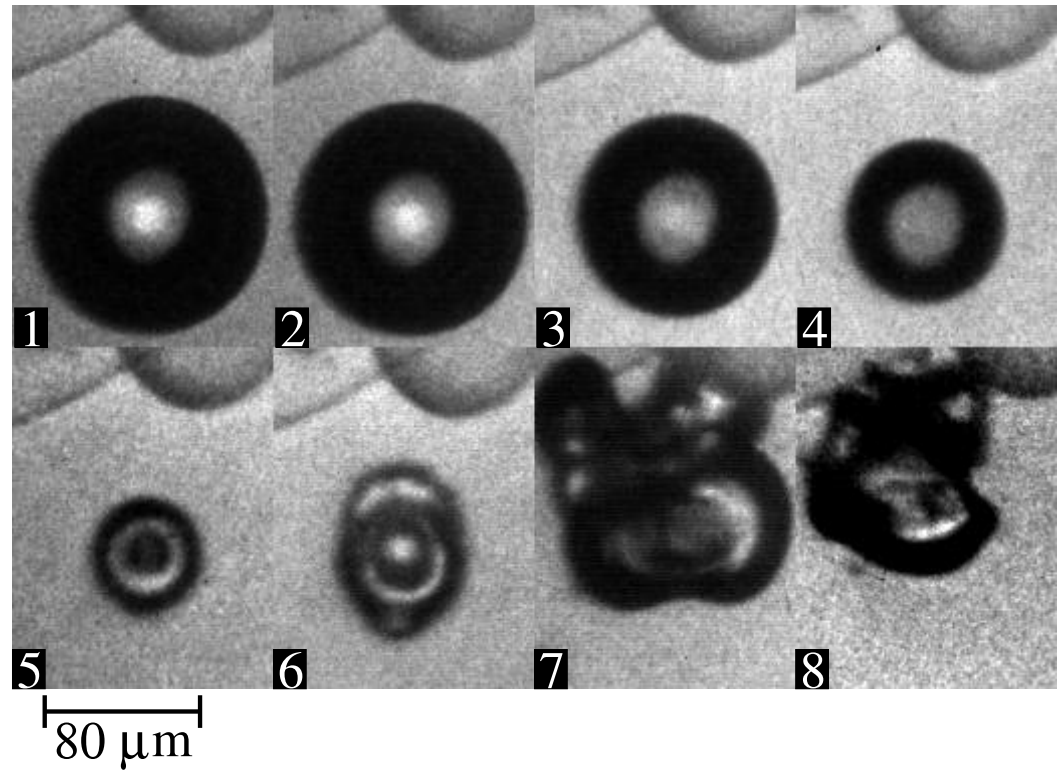

Figure 4.14: Bubble moving toward rigid boundary and splitting after multiple oscillations. The shock wave is incident from below. Frame 1 starts $\approx 0.4 \mu \mathrm{s}$ before shock wave passage. The exposure time is $100 \mathrm{~ns}$. Interframe times are 500 ns up to frame 6 , and $20.1 \mu$ s afterwards.

rigid boundary of the salt grain. The fragments remain close together and accumulate on the surface of the salt crystal. Subsequent application of shock waves will induce a collapse whose direction is governed by the rigid boundaries of the petri dish and the salt grain. Such a behavior can be seen in Fig. 4.15. In the first frame a bubble is located directly at the surface of the salt grain. After shock wave passage the bubble collapses and rebounds. It squeezes into the small cleft between petri dish and salt grain, thereby enlarging its visible area. The movement of the bubble into the cleft indicates a flow towards the intersection of the rigid boundaries forcing the bubble into the cleft.

Fig. 4.16 shows a bubble surrounded by three boundaries, which are composed of two flat faces of salt crystals and the bottom of the petri dish. The initial distance between bubble and salt crystal surfaces is about $28 \mu \mathrm{m}$. The bubble collapses first onto the petri dish glass bottom displaying again the torus-shaped form similar to Fig. 4.5. During rebound the bubble touches the salt crystals on both sides. Flow in direction of the salt grains is therefore strongly impeded upon second collapse. However, liquid flow is still possible through the channel indicated by the arrows in frame 6 of Fig. 4.16. This flow leads to a splitting of the bubble with two fragments remaining at the salt crystal's surfaces. 


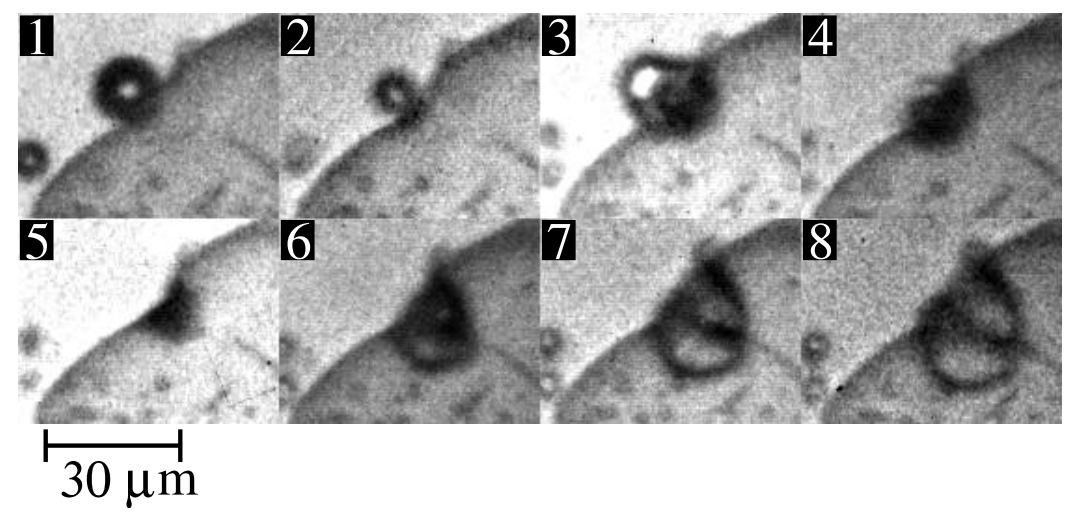

Figure 4.15: Bubble collapse and motion into the cleft between salt crystal and petri dish after shock wave application. The shock wave is incident from below. Frame 1 starts $\approx 0.3 \mu$ s before shock wave passage. Exposure and interframe times are $100 \mathrm{~ns}$ and $500 \mathrm{~ns}$, respectively.

Both fragments show structures similar to the counter jet known from torus collapses on boundaries [97]. Rigid boundaries can therefore be seen as competitors which attract the bubble. The boundary which imposes the strongest impediment to fluid flow will be most successful. A similar behavior of larger spark-created bubbles between two rigid boundaries has been observed by Chahine [104]. In the present case however, a third boundary, the plane of the petri dish, is predominant during the first collapse. After rebound an effect of the petri dish cannot be assessed due to the top view setup of the microscope. It is assumed that the collapse is directed into the two corners between salt grains and petri-dish. The attraction of collapse direction to corners has also been reported for laser created bubble near two perpendicular walls [14].

The collapse behavior in a similar situation with multiple boundaries can be seen in Fig. 4.17 where a bubble of approximately $150 \mu \mathrm{m}$ initial diameter is surrounded by four salt crystals. The first image was taken $\approx 1 \mu$ s after shock wave passage. The bubble faces four rigid boundaries including the plane surface of the petri dish. All of the boundaries take effect on the bubble dynamics. Due to the restriction on the flow the bubble collapses aspherically, staying elongated at the points facing the boundaries. Looking from an angle normal to the petri dish surface the bubble exhibits a triangular form upon collapse. Elongation is strong where the initial distance to boundaries is small. A triangularly shaped jet seems to hit the glass surface, seen from below, but no jet flow is hitting the more distant boundaries imposed by the salt grains. The bubble preserves an attached channel to the salt crystal it was in contact with before shock wave passage. The 


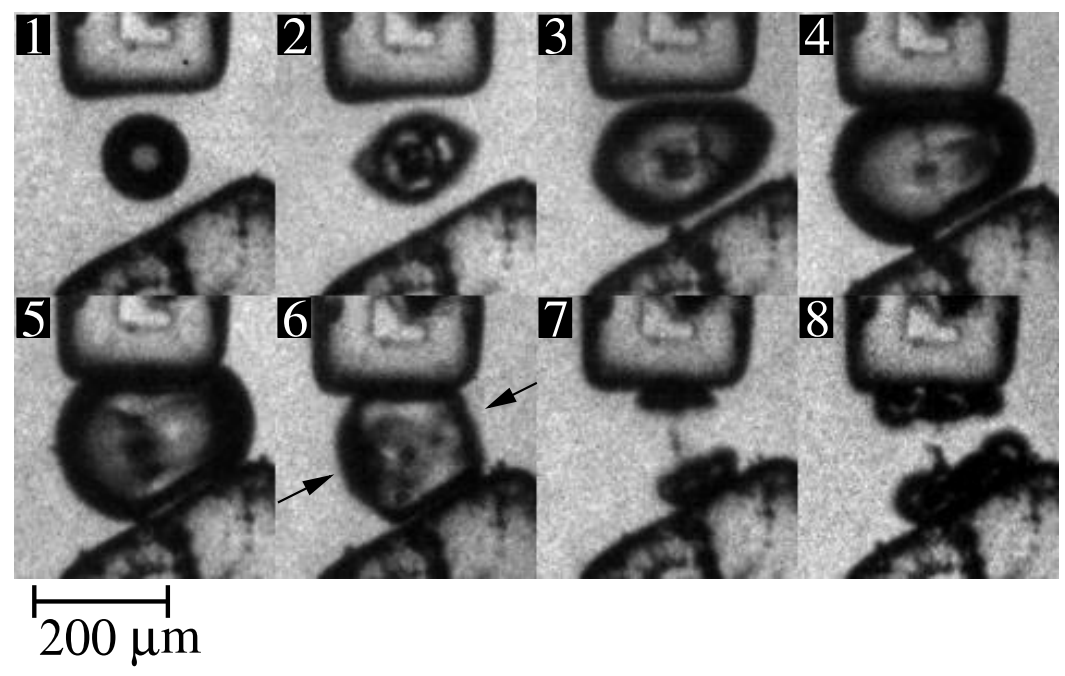

Figure 4.16: Bubble collapsing aspherically between two salt crystals. The shock wave is incident from below. Frame 1 starts $\approx 0.9 \mu$ s before shock wave passage. Exposure and interframe times are $1 \mu \mathrm{s}$ and $7 \mu \mathrm{s}$, respectively.

smallest salt grain to the left is slightly moved within the flow field towards the bubble during collapse.

\subsection{Particle disruption}

The results presented so far only dealt with the influence of rigid boundaries on bubble dynamics. Let us now turn to the effect of bubble collapse and expansion on nearby salt crystals. Occasionally the bubble dynamics in the vicinity of cracks can cause a rupture of salt crystals. Figures 4.18, 4.19, and 4.20 show a grain of salt after shock wave exposure with large interframe times of $31 \mu \mathrm{s}, 41 \mu \mathrm{s}$, and $81 \mu \mathrm{s}$, respectively. Bubble collapse and rebound at the rigid boundary lead to a fragmentation of the crystals. The destruction might be caused by the jet, which occurs during the aspherical bubble collapse and is directed toward the salt grain. It is also possible that the rapid expansion of gas pockets located within cracks of salt crystals induces shear forces, which break the crystals apart. In the experiments fragmentation was always accompanied by bubble activity, which strongly suggests that tensile forces of the primary shock wave are not responsible for the destruction of salt crystals in this setup.

It is very likely that similar cavitation effects contribute to shock wave and ultrasound 


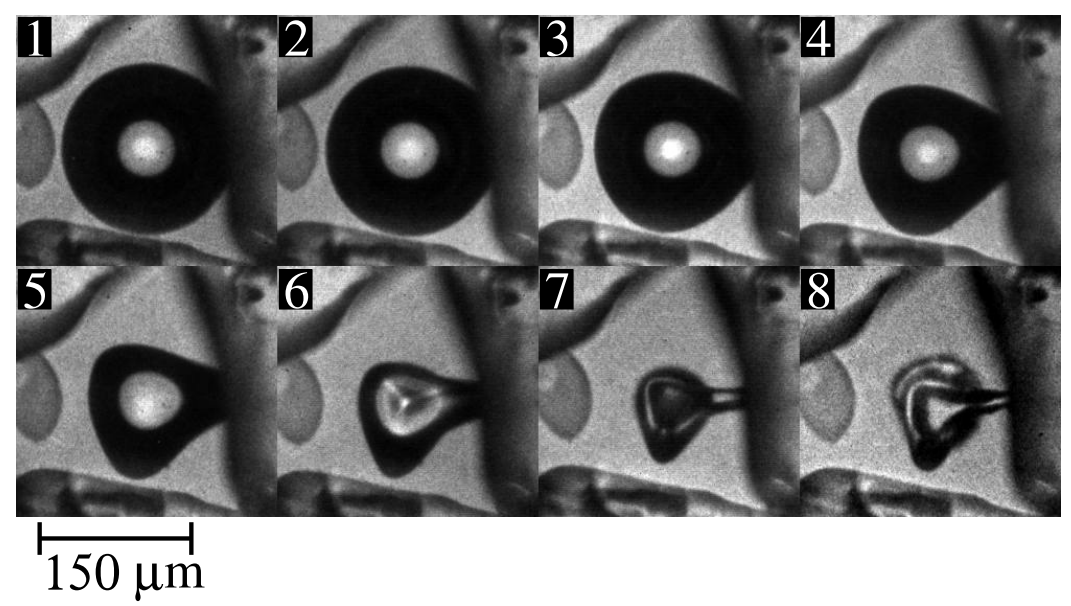

Figure 4.17: Bubble collapsing aspherically between salt crystals. The shock wave is incident from below. Frame 1 starts $\approx 0.3 \mu$ s before shock wave passage. Exposure and interfame times are $100 \mathrm{~ns}$ and $500 \mathrm{~ns}$, respectively. The bubble tends to elongate in the direction of rigid boundaries.

cleaning applications. Especially for continuous wave or multiple shock wave applications bubbles may first migrate towards solid objects and subsequently cause erosion due to aspherical collapse on the boundary. Cavitation induced damage similar to that observed in this paper might also add to the disintegration of renal calculi in shock wave lithotripsy $[73,75,65,66]$. However, direct interactions with the shock wave seem to play a major role in lithotripsy where the positive and negative pressure amplitudes, $p_{+} \approx 40 \mathrm{MPa}$ and $p_{-} \approx-10 \mathrm{MPa}[66,67]$, are much larger than in the experiments presented in this chapter.

\subsection{Conclusion}

The dynamics of shock-wave excited microbubbles has been investigated with high-speed recording methods. Initial radii of the bubbles before shock wave application were between 5 and $150 \mu \mathrm{m}$. The observed radius time curves have been compared to a numerical spherical single bubble model, which resulted in reasonable agreement. Single bubbles next to one or more rigid boundaries imposed by the petri dish and salt grains have been observed to collapse aspherically with partly complicated shape. Jet formation and torus collapse could be resolved for bubbles adjacent to one boundary, and sometimes surface instabilities with daughter bubble emission occurred during rebound. Bubble migration 


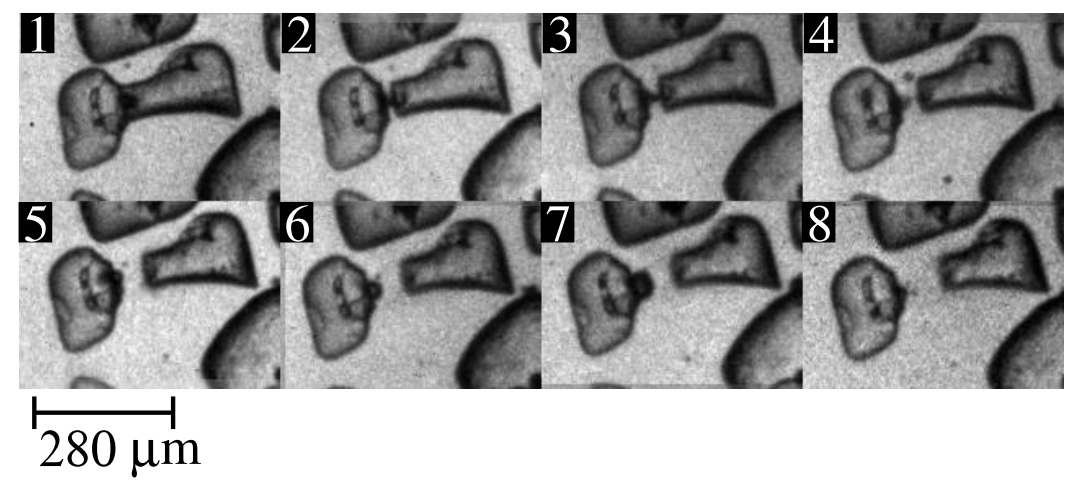

Figure 4.18: Bubble dynamics at a crack leading to separation of a salt crystal. The shock wave is incident from below. Frame 1 starts $\approx 0.9 \mu$ s before shock wave passage. Exposure and interframe times are $1 \mu \mathrm{s}$ and $31 \mu \mathrm{s}$, respectively.

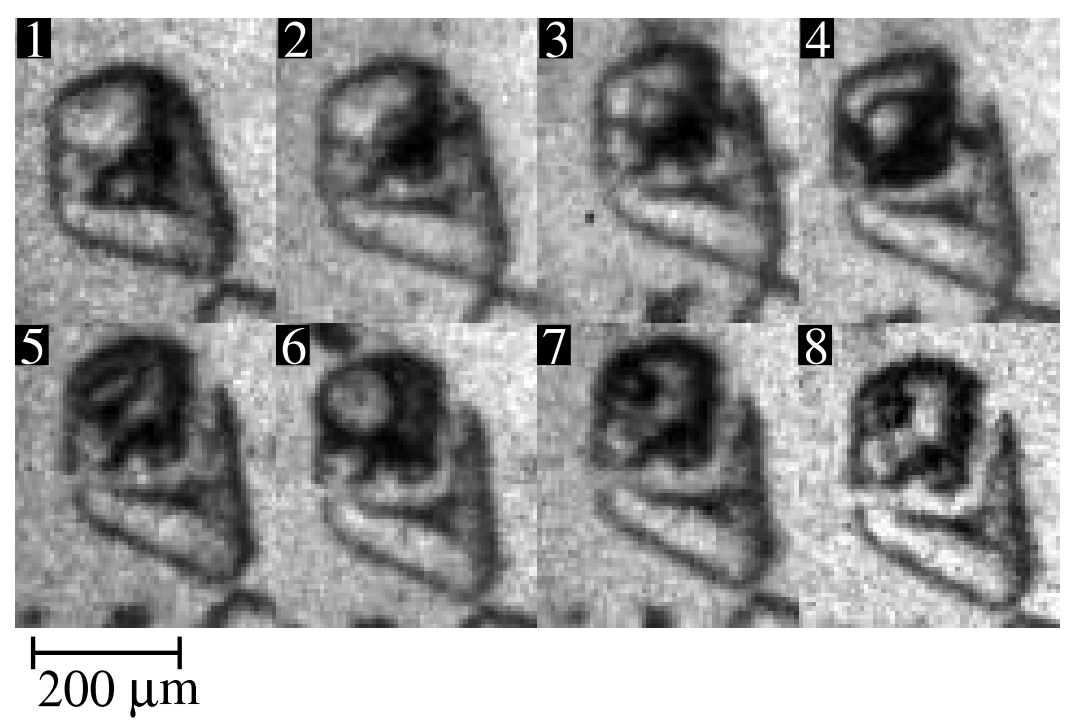

Figure 4.19: Breaking of a salt crystal by bubble action. The shock wave is incident from below. Frame 1 starts $\approx 4 \mu$ s before shock wave passage. Exposure and interframe times are $1 \mu \mathrm{s}$ and $41 \mu \mathrm{s}$, respectively. 


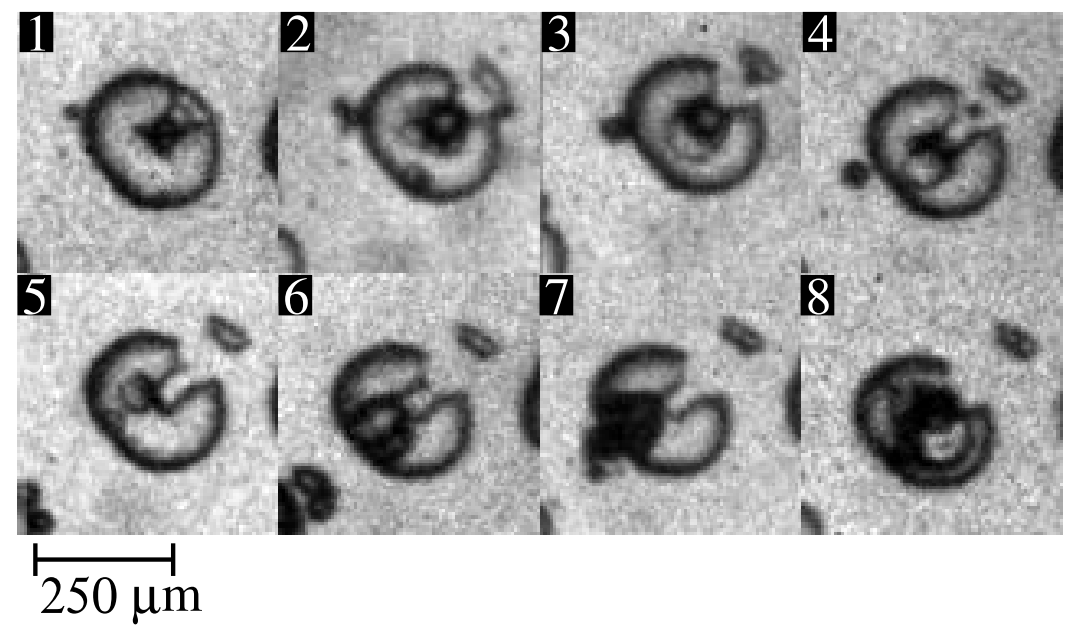

Figure 4.20: Breaking of a salt crystal by bubble action. The shock wave is incident from below. Frame 1 starts $\approx 4 \mu$ s before shock wave passage. Exposure and interframe times are $1 \mu \mathrm{s}$ and $81 \mu \mathrm{s}$, respectively.

towards boundaries and even into cracks or clefts of the salt crystals have been observed. Multiple boundaries can lead to bubble splitting or to deformation with the tendency to jet towards each interface. Then, the dynamics is governed by the restriction on fluid flow imposed by each rigid boundary leading to a "jet competition". Multiple bubbles showed splitting during collapse and merging in the rebound phase of motion, which in turn can cause agglomeration. Translation can add to the clustering tendency of many bubbles after the shock wave passage. Bubbles adjacent to salt crystals have been observed to be able to break the particles due to their rapid dynamics. Similar destructive behavior might also be involved in ultrasonic cleaning applications under shock or continuous wave excitation. The possible role of microcracks and cavitation in stone fragmentation remains to be clarified. 


\section{Chapter 5}

\section{Pressure wave excited contrast agent bubbles in the vicinity of cells}

After general aspects of the dynamics of microbubbles have been demonstrated in the preceding chapter now results are presented, which reveal the interaction of microbubbles with cells in vitro. Contrast agent bubbles located near cells have been excited by a spark created pressure wave. The bubble dynamics and its effects on cells have been recorded with high-speed imaging techniques [127].

It is an obvious approach to use contrast agents as inceptors for microbubbles because they are also used in ultrasonic imaging and are therefore relevant for medical sciences. Furthermore, the position of contrast agent bubbles with respect to cells can be observed under a microscope prior to pressure wave excitation. The use of contrast agents constitutes a step further down in size compared to the bubbles created in the preceding chapter. The diameter of Levovist contrast agents which are utilized in this setup ranges from approximately 1 to $8 \mu \mathrm{m}$.

\subsection{Materials and methods}

The experimental setup depicted in Fig. 5.1 is similar to the setup described in Chapter 4. Main differences lie in the illumination of the scene and the generation of the pressure waves. A petri-dish (Nunc) containing cells and contrast agent was mounted on an inverted Microscope (Axiovert 135 TV, Zeiss). Two different objectives were used:

1. magnification $100 \times$, oil immersion, numerical aperture: 1.25 


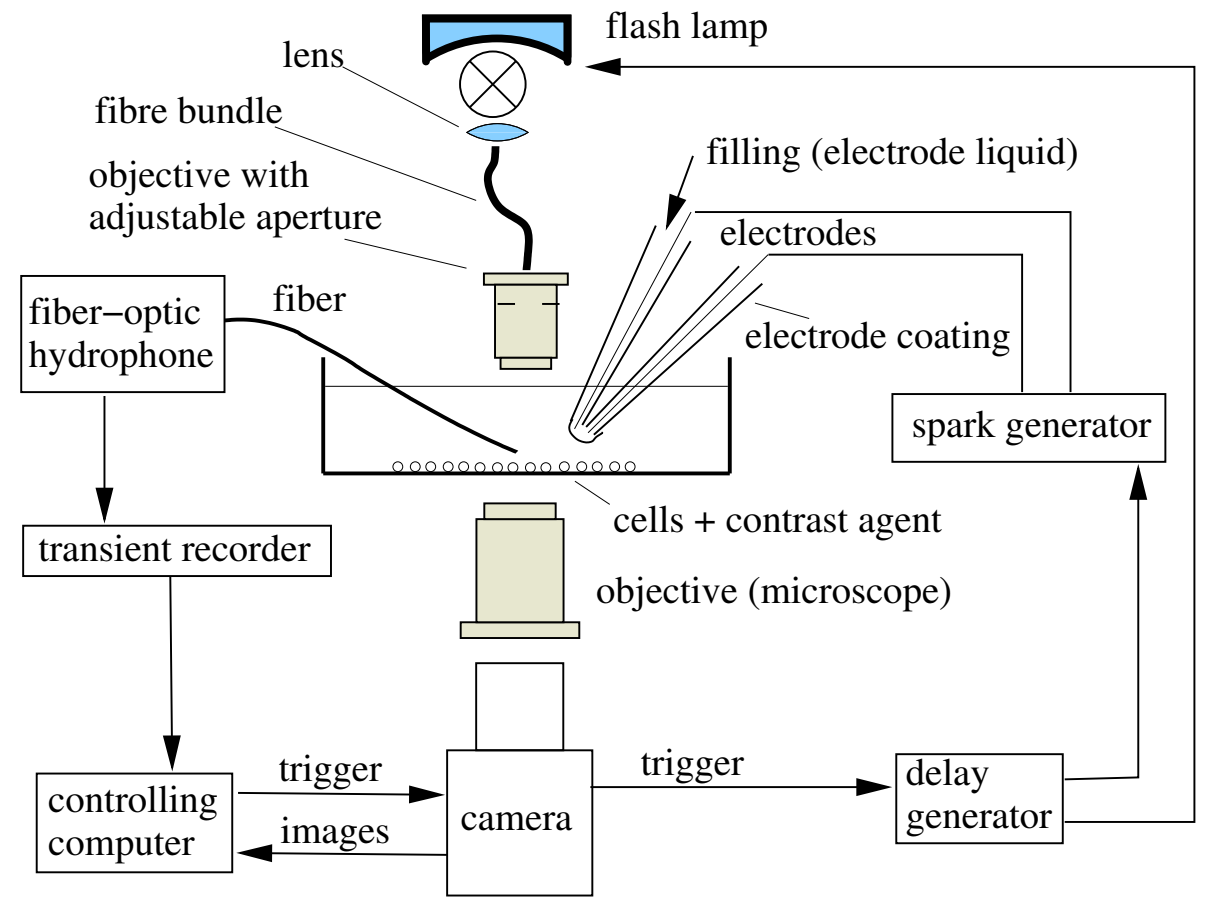

Figure 5.1: General setup for the excitation of Levovist contrast agents and subsequent observation of bubble dynamics.

2. magnification $63 \times$, air, numerical aperture: 0.85 .

In some cases a $1.6 \times$ zoom was applied to achieve the desired magnification. Images were again captured with a high-speed Imacon ICCD-camera. However, because of the larger magnification used in this setup it was necessary to increase illumination brightness. Therefore, the light of the xenon flash lamp was coupled into a fiber bundle. An objective with variable aperture was used to illuminate the observed area. To improve image quality a hole, approximately $1 \mathrm{~cm}$ in diameter was milled in the polystyrol bottom of the petridish. The hole was sealed by a cover slip, which was glued to the bottom of the petri dish using a silicone rubber compound (RTV615, GE Bayer Silicones).

\subsubsection{Cell culture and contrast agent}

For the experiments Levovist (Schering AG, Germany) contrast agent and normal rat kidney (NRK) fibroblast cells were used. The cells were grown at $37^{\circ} \mathrm{C}$ in a $5 \% \mathrm{CO}_{2}$ environment in Dulbecco's modified Eagle's medium (DMEM) supplemented with 10\% fetal calf serum and $1 \%$ penicillin/streptomycin. Prior to being exposed to pressure waves 
Kap. 5 Pressure wave excited contrast agent bubbles in the vicinity of cells

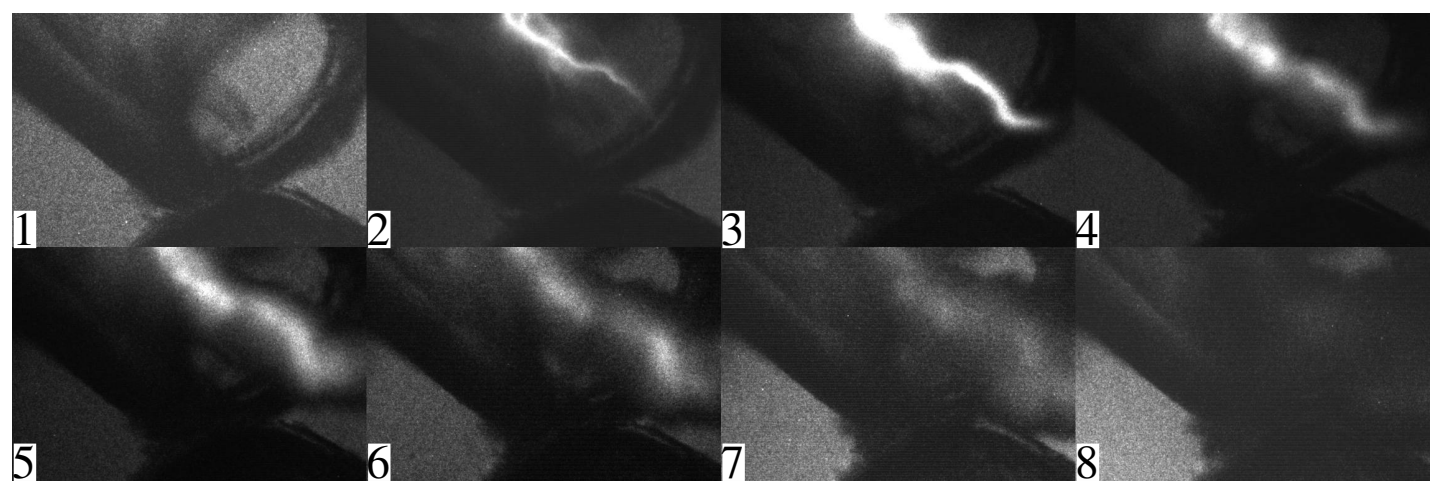

Figure 5.2: Image sequence showing spark generation by a high voltage pulse in water. The electrodes are hidden in plastic covers. The circular structure depicts the opening of such a cover at the tip. The width of a single frame is $\approx 800 \mu \mathrm{m}$. Exposure and interframe times are $500 \mathrm{~ns}$ and $500 \mathrm{~ns}$, respectively.

the cells were trypsinated, resuspended in $1.5 \mathrm{ml}$ medium, and placed in the Nunc petridish with the inserted cover slip. Contrast agent was added by dissolving approximately $3 \mathrm{mg}$ Levovist powder into the medium.

\subsubsection{Pressure excitation of contrast agents}

The pressure waves were created by an electrical breakdown in water. High voltages were provided by a spark generator rapidly discharging a capacitor across a spark coil. Coated wires with $\approx 50 \mu \mathrm{m}$ diameter were used as spark electrodes. In most cases the electrodes have been placed inside a silicone tube filled with water to be separated from the cell suspension. Upon discharge a plasma is created in the liquid, which leads to shock wave and bubble formation. Amplitudes of the pressure waves at the point of observation were measured with the fiber optical hydrophone (FOPH 300) described in Section 4.1.1 and were read out by a digital oscilloscope (TDA784A). Timing of camera, flash lamp, and spark generator was controlled by using a delay generator (DG535). The trigger was initiated by the camera. Figure 5.2 shows the formation of a voltage induced plasma in water. After plasma generation we see, starting in frame 4, a bubble developing in the liquid. This process is similar to the generation of laser induced cavitation described in Section 4.1.1. However, it lacks the spherical symmetry of the latter. 


\subsection{Bubble dynamics and cell deformation}

The spark generated pressure signal depends strongly on the distance of the electrodes and the ionic concentration of the medium surrounding the electrodes. Figure 5.3 (a) shows a typical hydrophone signal recorded with the same setup as the image sequence displayed in Fig. 5.3 (c). The high amplitude signal during the first microsecond in Fig. 5.3 (a) is caused by the strong electromagnetic noise generated by the voltage discharge and cannot be attributed to pressure variations. Thereafter, the signal consists of a fast oscillating part with amplitudes up to $5 \mathrm{MPa}$ and a longer lasting tensile pulse just slightly higher than the background noise. The oscillations are probably caused by reflections at low acoustic impedance boundaries. Although the key characteristics of the pressure signal remain the same for each spark generation, the exact number and amplitudes of the pressure peaks vary from spark to spark. This is to be expected since the electrical breakdown is a statistical process and additionally each voltage discharge may cause damage to the electrodes and therefore slightly change the conditions for spark generation. Also it can be seen in high-speed images that the path of plasma formation varies for each voltage discharge.

The theoretical radius-time curve of a bubble with $1 \mu \mathrm{m}$ initial radius has been calculated using the Keller-Miksis model of equation (4.1) and the results have been compared with the experimental data (Fig. 5.3). The exciting pressure in the model has been approximated from the hydrofone measurement using a nonlinear least squared method and is depicted in Fig. 5.3 (a) and (b). All other parameters in the calculations are the same as described in Section 4.1.3. The plot can only be regarded as a qualitative comparison, since the Keller-Miksis model does not include boundary surface effects or the coating of the contrast agent. Also, the excitation used is only an approximation and may differ from the true pressure wave. However, the comparison strongly suggests, that the low amplitude tensile pulse and not the fast high amplitude signal is responsible for the large expansion of the contrast agent bubble. To rule out any influence by the high amplitude signal the ionic concentration of the medium surrounding the electrodes has been increased, resulting in a decreased maximum voltage due to the lower resistance and also in a decreased peak amplitude of the high pressure signal generated by the electrical breakdown. For high ionic concentrations bubbles form between the electrodes before plasma formation. The spark is then initiated inside these bubbles where the threshold for electrical breakdown is much lower $(\approx 3 \mathrm{MV} / \mathrm{m}$ for air) than in water $(\geq 20 \mathrm{MV} / \mathrm{m})$ [128]. Such a scenario is depicted in Fig. 5.4 for a voltage discharge between two steel electrodes in 


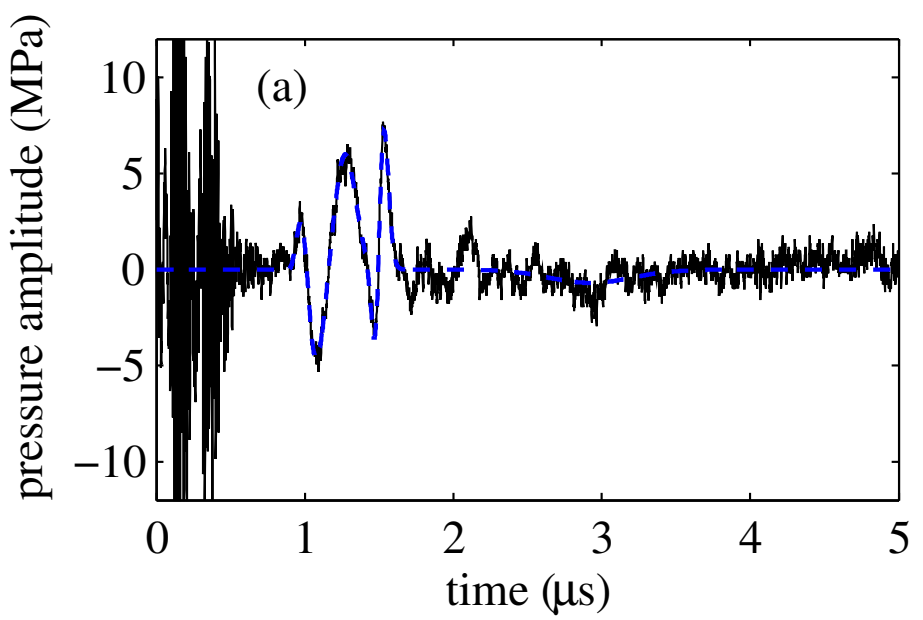

(c)

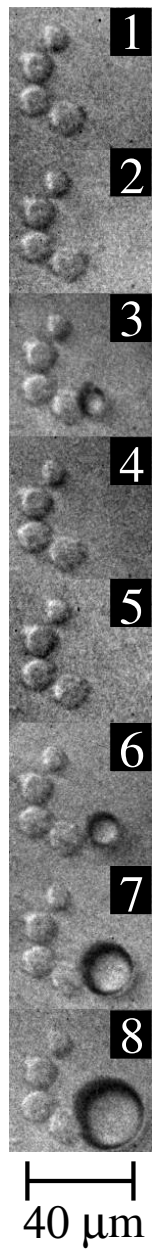

Figure 5.3: (a) Pressure as measured by the optical hydrophone (black curve) and fitted pressure (dotted blue curve). (b) Calculated and measured radius time curves of the contrast agent bubble from (c) (black curve, data points) and the input pressure function fitted from the measured pressure signal of (a) (dotted blue curve). (c) Optical observation of a contrast agent bubble exposed to a pressure wave. The bubble is located next to one of four cells, hardly visible in frames 1, 2, 4, and 5. Finally it grows to a size much larger than the cells. Optical exposure and interframe times are 150 and $600 \mathrm{~ns}$, respectively. 


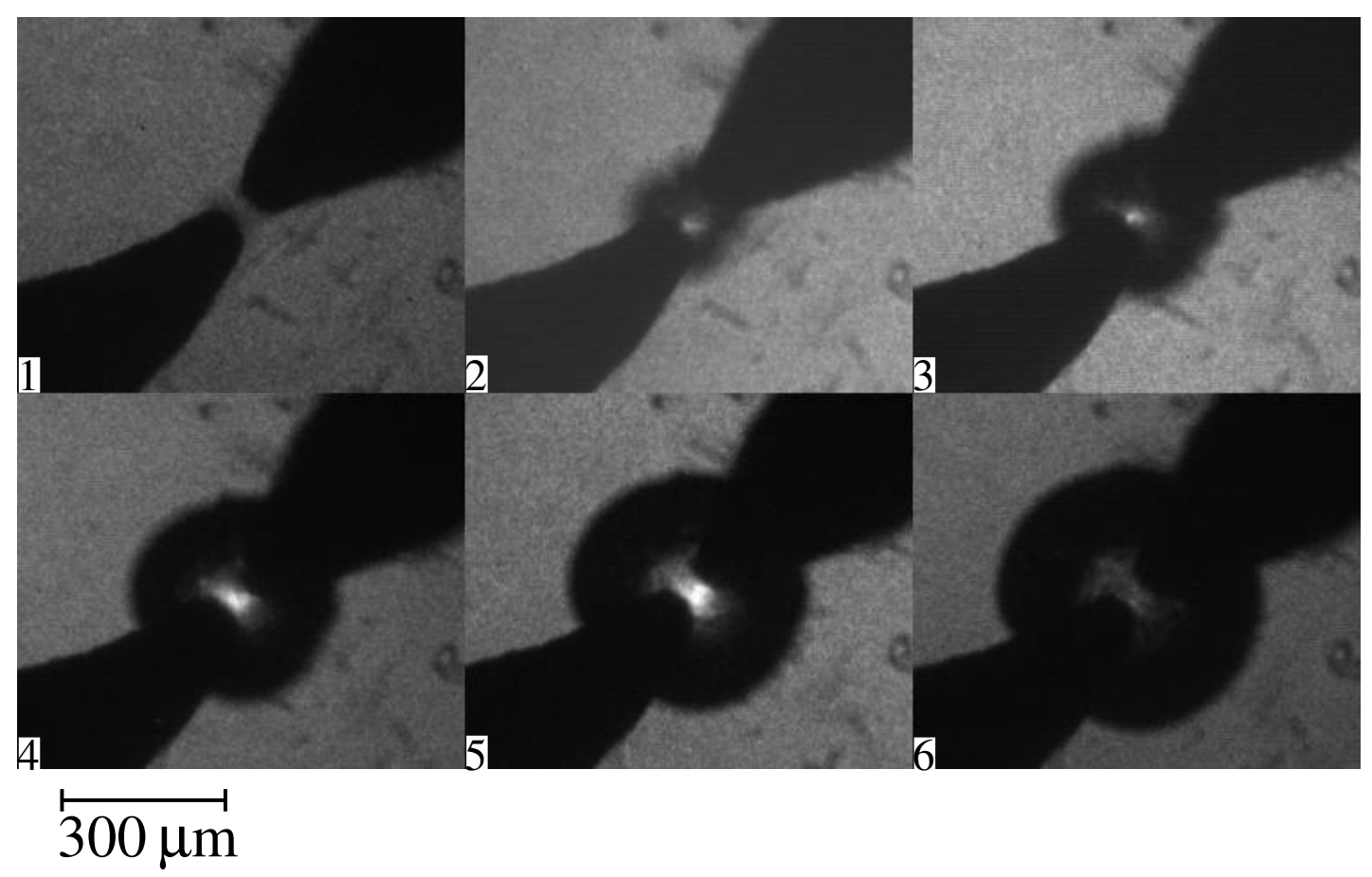

Figure 5.4: Electrical breakdown between two steel electrodes in salt water. The discharge takes place inside the growing bubble. Exposure and interframe times are $3 \mu \mathrm{s}$ and $3 \mu \mathrm{s}$, respectively.

salt water. In normal tap water, the ionic concentration is smaller so that the breakdown occurs already in the liquid phase as shown in Fig. 5.5.

Figure 5.6 shows the expansion phase of a contrast agent bubble near a single cell. The tension part of the pressure pulse in this case is at the resolution limit of the hydrophone $(\approx 1 \mathrm{MPa})$. The contrast agent bubble has an initial diameter of $2.0 \pm 0.5 \mu \mathrm{m}$ in the first frame and expands up to a size of $62 \pm 3 \mu \mathrm{m}$ across its longest section in frame 6 . Bubbles which are not in the vicinity of a cell or any other obstacles tend to become spherical upon expansion. It is shown in Fig. 5.6 that the bubble expansion is perturbed by the cell resulting in a kidney shaped form of the bubble. The cell on the other hand is affected in two ways. 1) It is translated away from the bubble center, and 2) it is deformed slightly yielding an oval shaped form with the shorter axis directed to the bubble center. The longer axis is extended by $\approx 8 \%$ while the shorter axis is reduced by $5 \%$ to $40 \%$. Only an approximate range can be given since the bubble wall inhibits sight on the left edge of the cell. Assuming an oblate ellipsoidal form of the cell after deformation and assuming the volume to remain constant we get a reduction of the foreshortened axis of $\approx 14 \%$. 


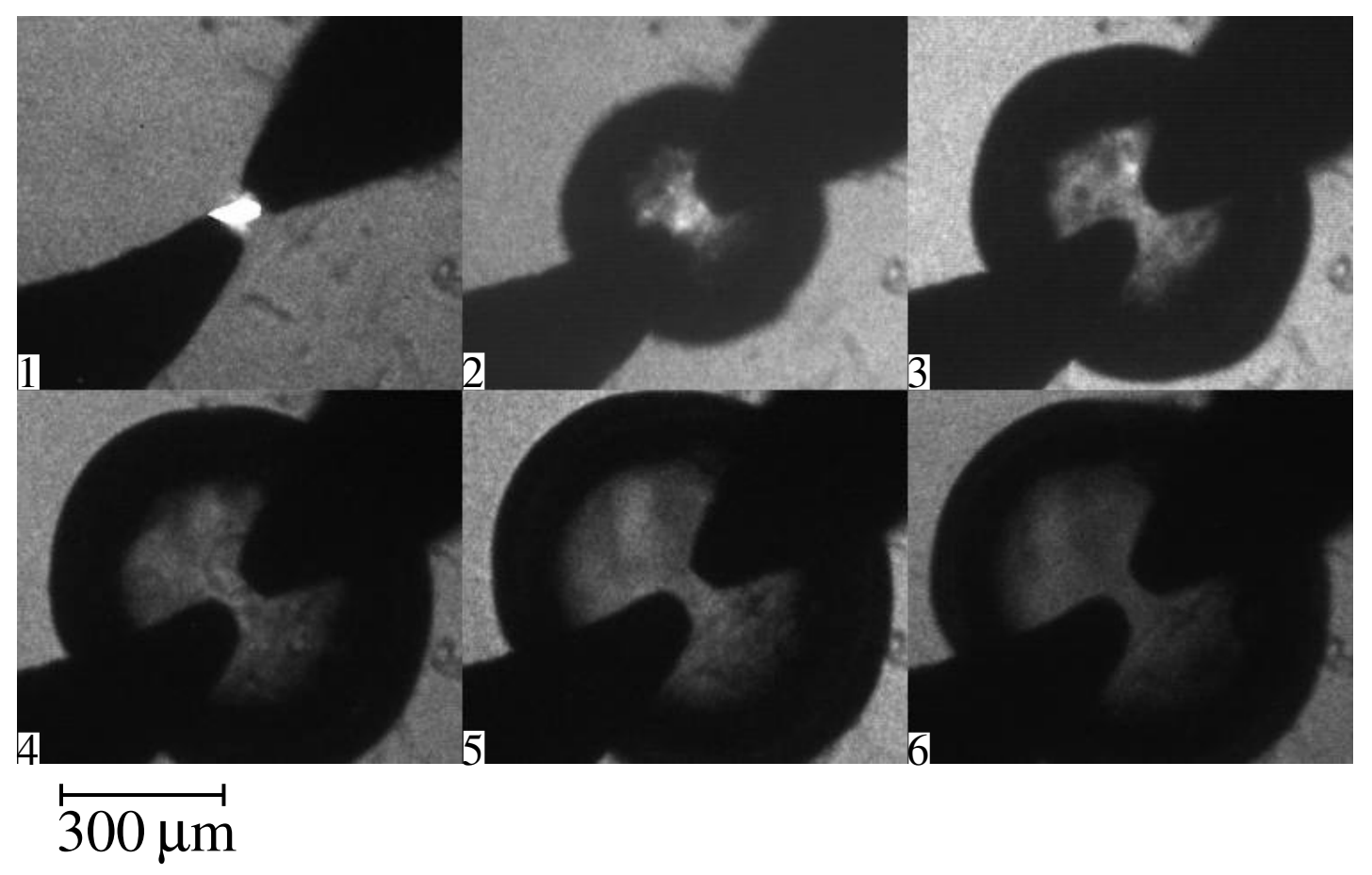

Figure 5.5: Electrical breakdown between two steel electrodes in tap water. The discharge takes place prior to bubble growth. Exposure and interframe times are $3 \mu \mathrm{s}$ and $13 \mu \mathrm{s}$, respectively. 


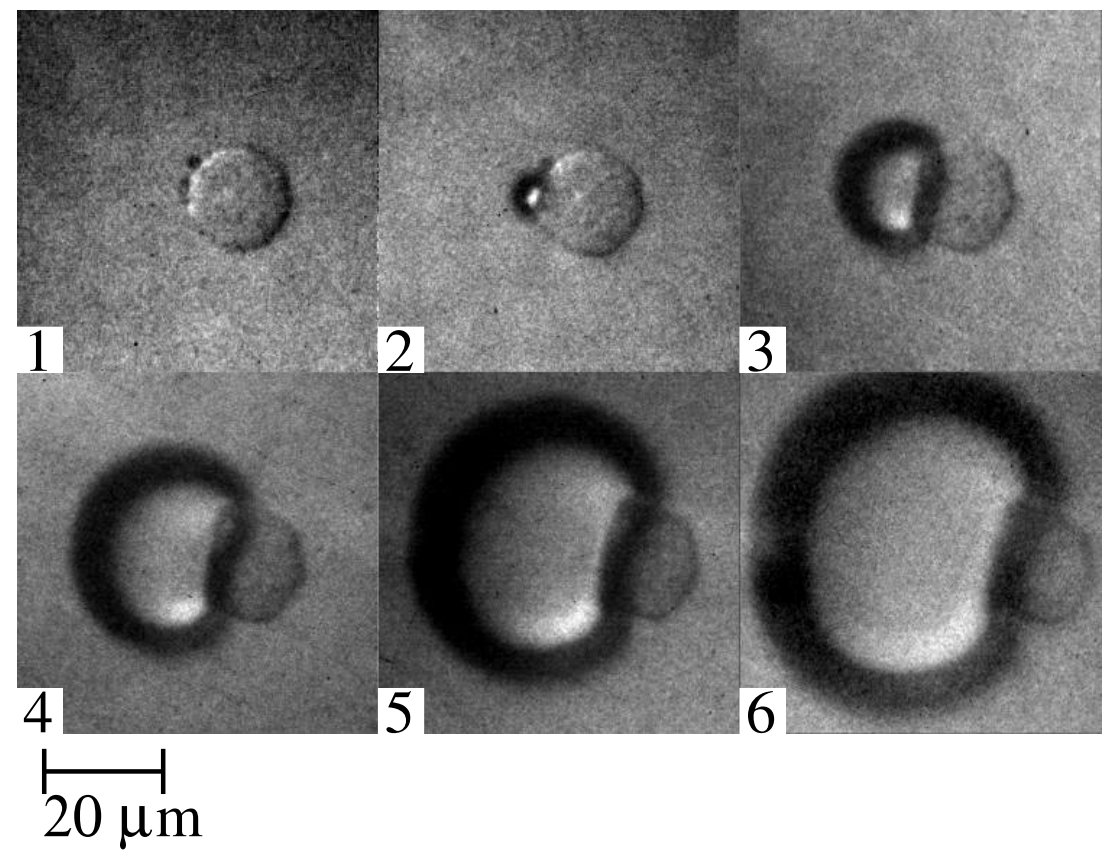

Figure 5.6: Expansion of contrast agent bubble adjacent to a cell. The pressure pulse is incident from the left. Exposure and interframe times are $200 \mathrm{~ns}$ and $800 \mathrm{~ns}$, respectively. 


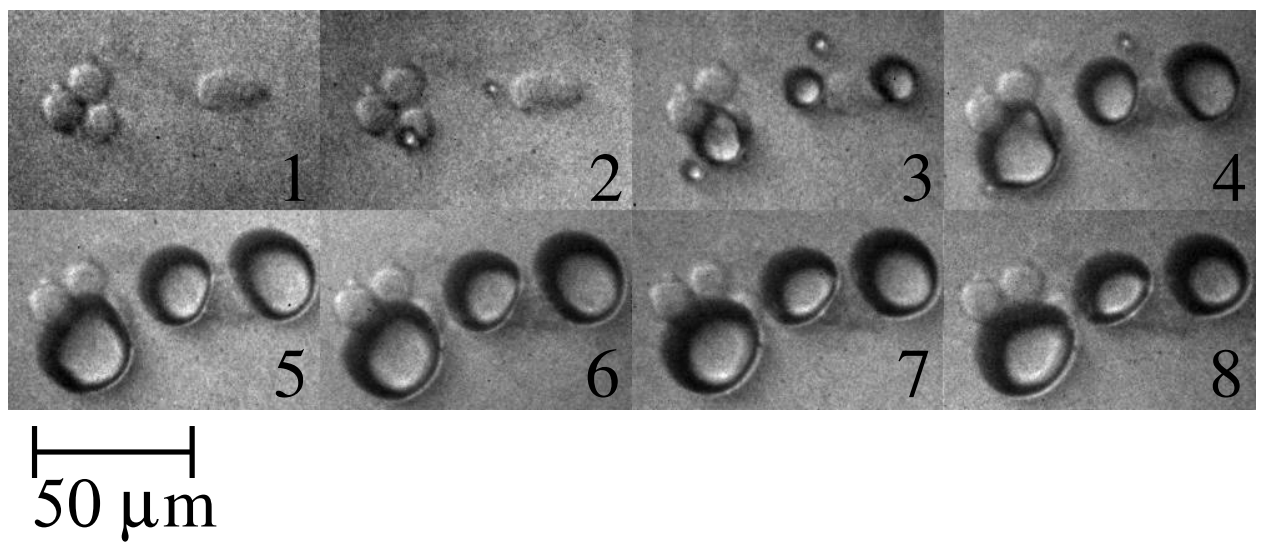

Figure 5.7: Expansion of contrast agent bubbles separating cells. The pressure pulse is incident from the left. Exposure and interframe times are $200 \mathrm{~ns}$ and $900 \mathrm{~ns}$, respectively.

This results in an areal strain of $\Delta A / A=1.2 \%$, which is below the critical strain for membrane rupture of red blood cells [129]. The small strain implies that the tension is not large enough to damage the lipid bilayer, although one cannot rule out the generation of higher strains localized at certain membrane regions such as the interface to the bubble. In any case however, expanding contrast agent bubbles are capable of separating cells in cell clusters, which is depicted in Fig. 5.7. Here the two lower cells of the three-cell cluster are pushed apart from each other by an expanding bubble.

The deformation of cells in the vicinity of a collapsing bubble is much stronger than during the bubble expansion phase. Figures 5.8 shows the collapse of an expanded bubble and its action on cells nearby. The pressure wave is incident from the left. In part (b) of the figure the relevant area has been enlarged and edges have been enhanced. Fig. 5.8 (a) depicts three bubbles during collapse phase. In the first frame we can see the contrast agents located at the cell membranes before pressure wave application. After expansion the topmost bubble collapses upon a cluster of three cells in the upper left corner of the images, see Fig. 5.8 (b). All of the three cells are strongly deformed upon collapse and at least the lower left cell seems to be visibly ruptured. In frame 6 the bubble displays a boomerang shape indicating a jet in the direction of the neighboring bubble. The jet was detected in almost every bubble collapse. There were three predominant factors, which affected the direction of the jet:

1) The vicinity to other bubbles: As shown in Fig. 5.8 bubbles tend to collapse in the direction of their closest neighboring bubble if their distance is short compared to their 

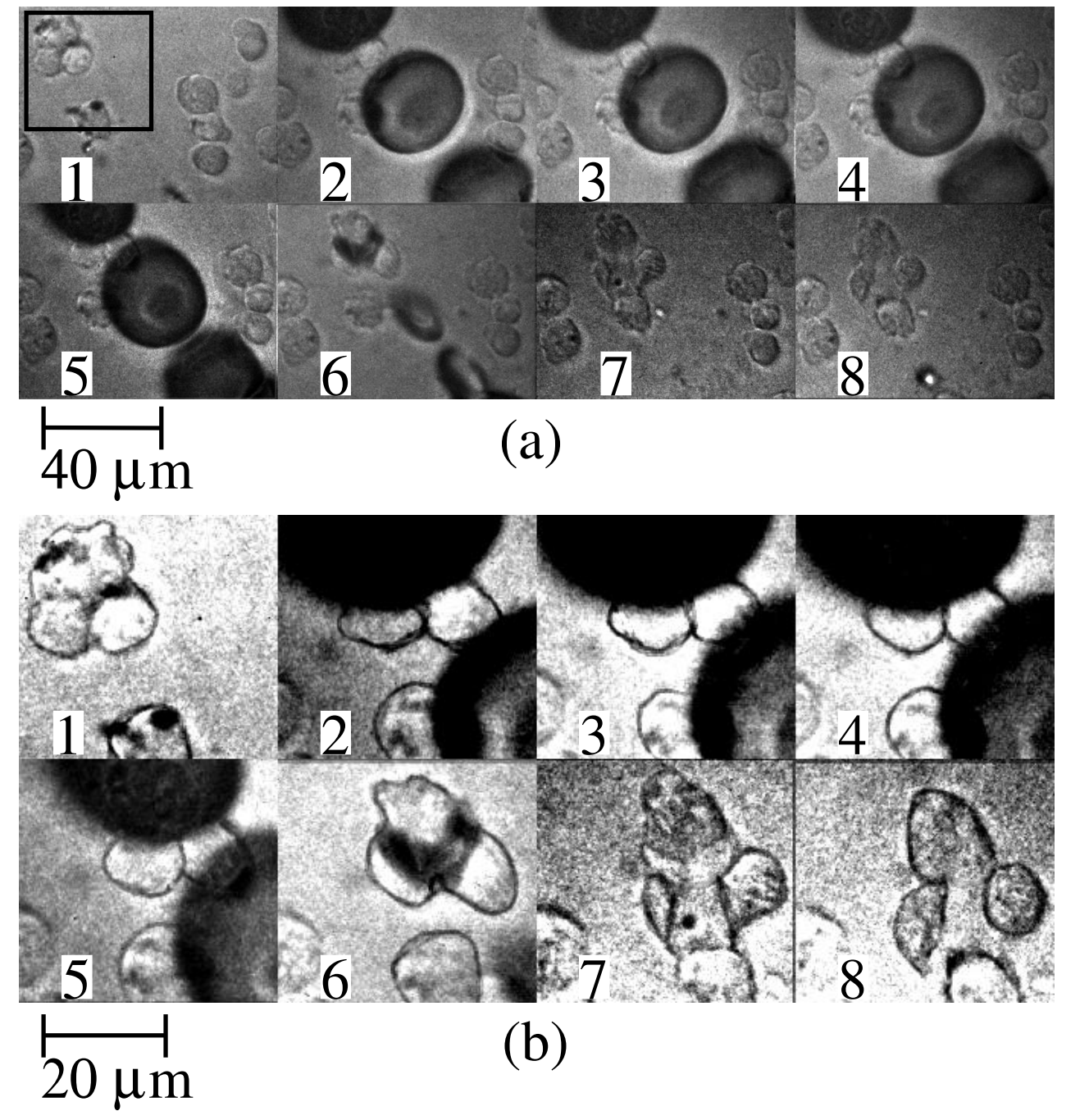

Figure 5.8: (a) Collapse of contrast agent bubbles. Exposure time is $200 \mathrm{~ns}$. The first frame displays the cells and contrast agent bubbles before pressure wave exposure. The second frame starts $\approx 10 \mu$ s after passing of the first pressure pulse. Subsequent interframe time is $1.2 \mu \mathrm{s}$. (b) Enlarged and image edited sequence of the framed region of (a). The pressure pulse is incident from the left. 


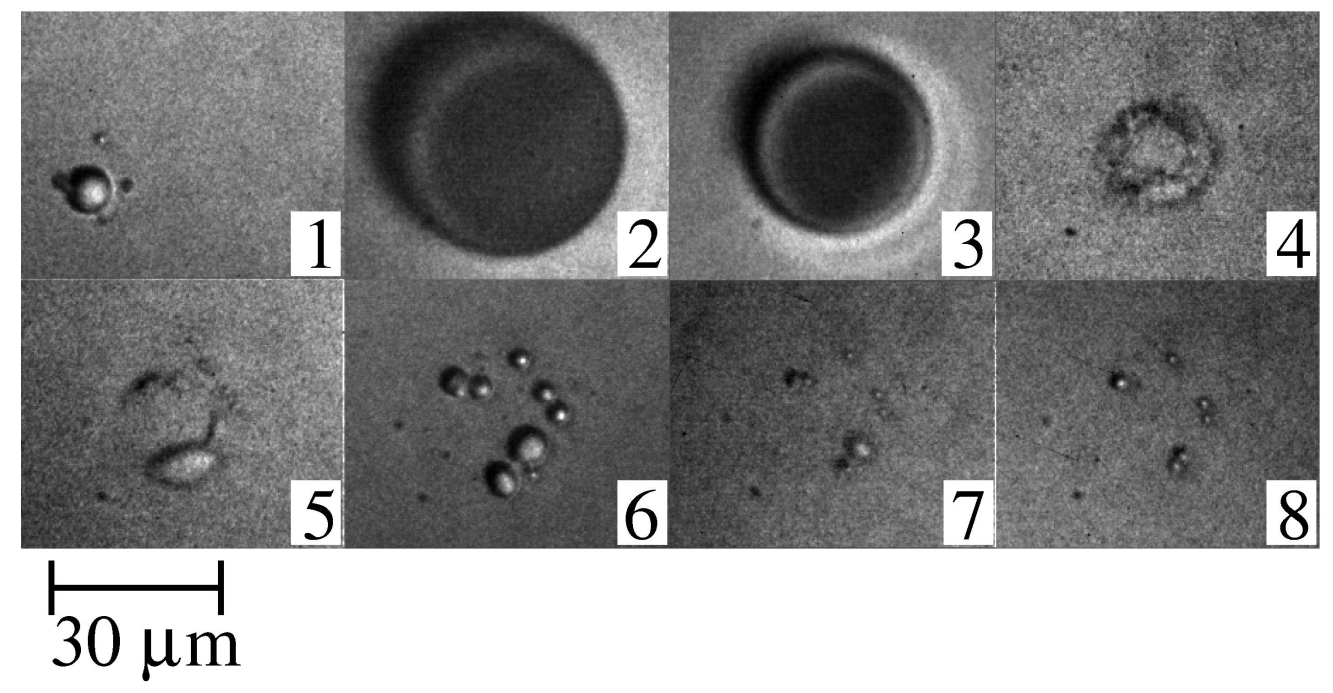

Figure 5.9: Contrast agent bubble collapsing aspherically onto a glass surface after pressure wave excitation. The first frame is taken before pressure excitation. The second frame starts after expansion of the contrast agent due to a tensile pressure pulse. Exposure and interframe times after frame 1 are $200 \mathrm{~ns}$ and $600 \mathrm{~ns}$, respectively. The pressure pulse is incident from the lower left.

maximum diameter and the proximate bubble is of the same size or larger. This effect is caused by resistance of flow, which results in an aspherical collapse and subsequent jetting similar to the collapse close to a rigid boundary.

2) The vicinity to a rigid boundary: In these experiments the rigid boundary was given by the glass cover slide in the bottom of the petri-dish. Sometimes a torus-like collapse as in Fig. 5.9 comparable to the collapse of larger laser or spark generated bubbles in the vicinity of rigid boundaries [16] was detected (see also Fig. 3.7).

3) The direction of the pressure wave: In the case where the influence of adjacent boundaries or other contrast agent bubbles is small the bubbles often tend to jet away from the pressure source upon collapse. Such a scenario is depicted in Fig. 5.10. Here, three already expanded bubbles are collapsing in the propagation direction of the pressure wave. Part (b) of the figure is an image edited enlargement of (a), which displays the deformation of two cells in the flow field induced by the collapse of the rightmost bubble.

The pressure wave directed collapse might be caused by the inhomogeneous flow field of the larger expanding bubble created by the spark electrodes. The effect was strongly enhanced when the tube which separated the electrodes from the cell medium was omitted. 

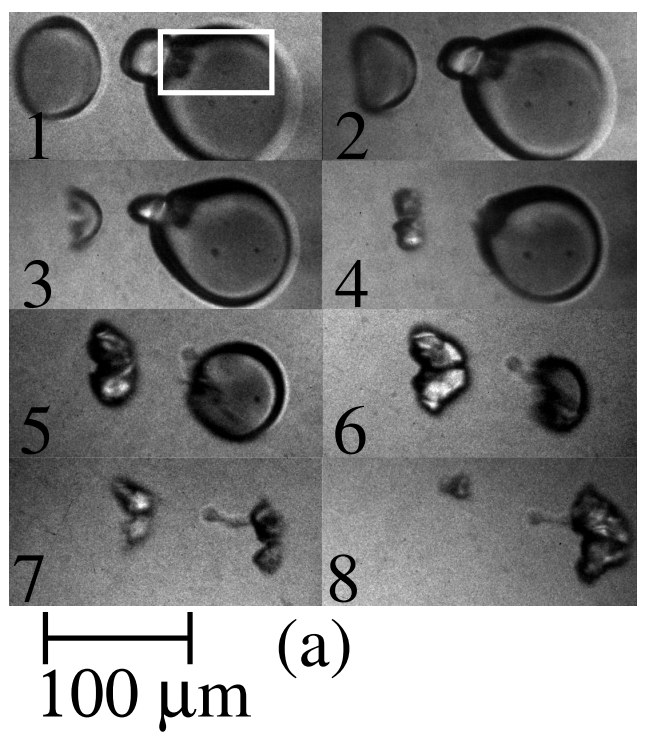

(a)

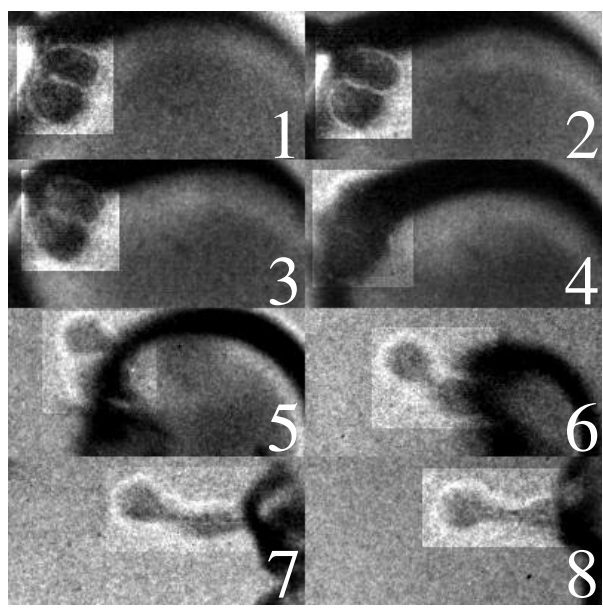

(b)

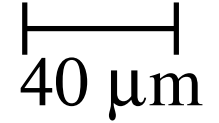

Figure 5.10: Collapse of contrast agent bubble. Exposure and interframe times are $200 \mathrm{~ns}$ and $600 \mathrm{~ns}$, respectively. The first frame starts $\approx 15 \mu$ s after passing of the first pressure pulse. Part (b) is an image edited enlargement of (a) showing two adjacent cells, which are strongly deformed during the collapse of the rightmost bubble. The pressure pulse is incident from the left. 


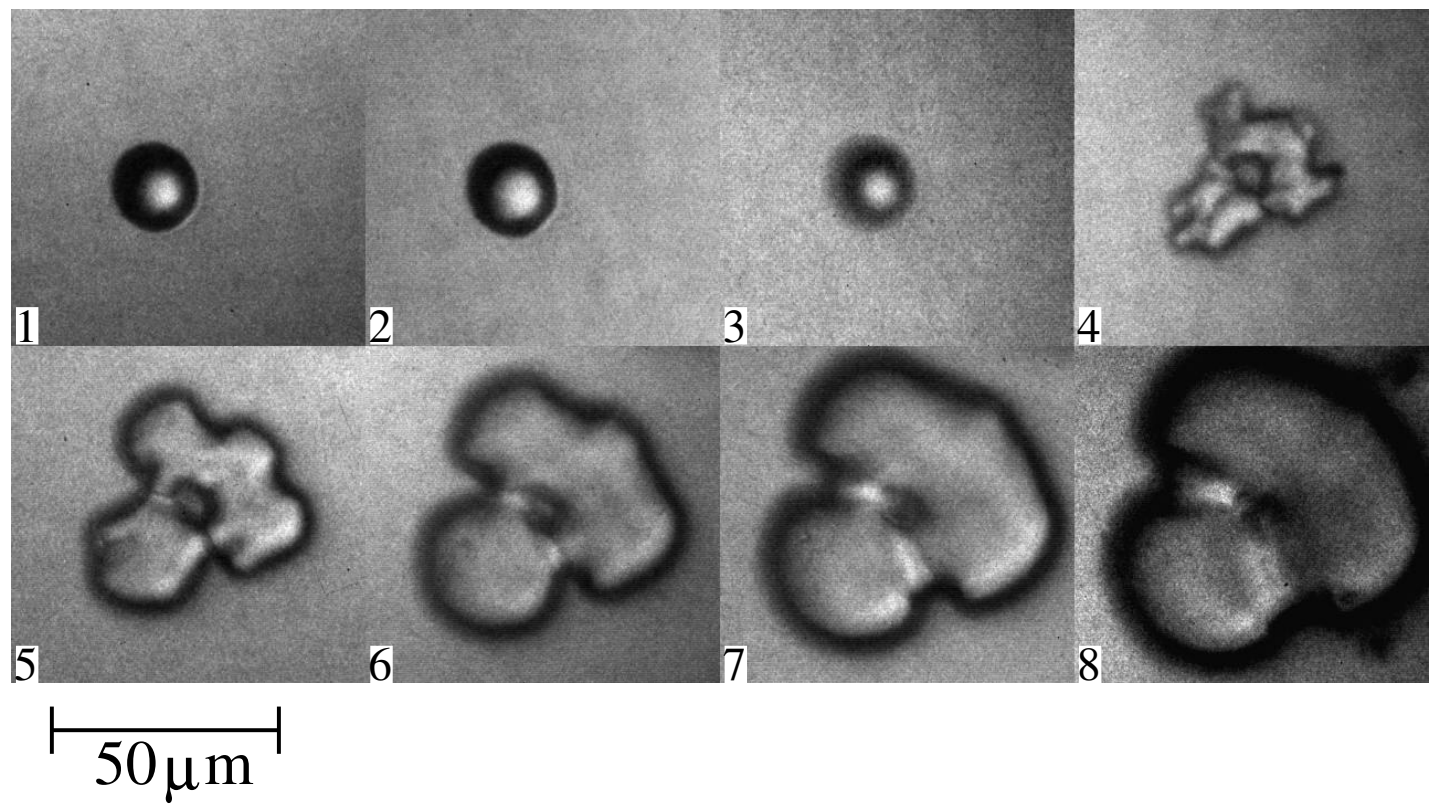

Figure 5.11: Collapse and rebound of a contrast agent bubble at the bottom of the petri dish after pressure wave excitation. The pressure wave is incident from the left. Exposure and interframe times are $100 \mathrm{~ns}$ and $300 \mathrm{~ns}$, respectively.

Usually collapse and rebound behavior was affected by both, the rigid boundary and the direction of the pressure wave. This can be seen in Fig 5.11, where a bird-foot shaped bubble appears after rebound similar to the bubble of Fig 4.10. The experiments yielded no indication that the collapse is also influenced by the cells in the vicinity of the bubble. This is important because a microjet directed at a cell might penetrate the membrane in a small area and lead to transient cell permeabilization. Such a process could be exploited to facilitate drug delivery. Although no indication of a cell directed bubble jet could be found, other mechanisms like rigid boundary collapse or the propagation path of the pressure wave might be used to direct a bubble jet towards a cell. For example, bubbles exposed to a lithotripter shock wave have been observed to develop a pin like jet directed in the propagation path of the pressure wave [130]. However, present techniques do not allow to assess membrane poration by bubble jets. To implement jet induced membrane poration by acoustic excitation the position of microbubbles needs to be controlled with respect to cells. Also the diameter of the bubble jet should be small compared to the cell to allow for reparable membrane permeabilization. Bubble-jets investigated so far do not fulfill these conditions. Due to the tensile part of the pressure pulse the contrast agent bubbles used in these experiments also grow to the same size of cells and larger. 
Therefore, a direct jet penetration of the membrane seems unlikely. However, this does not exclude transient and permanent membrane poration due to shear effects induced by expanding and collapsing bubbles.

Lokhandwalla and Sturtevant [129] analyzed the deformation of a cell subjected to an extensional flow field. They distinguished between an inertial and a viscous mode: In the inertial mode the tension on the membrane caused by inertial forces of the fluid is strong compared to the elastic tension of the cell membrane. The cell deformation is therefore governed by the fluid flow. An example of inertial mode is given in Fig. 5.10 where two cells are strongly stretched in the suction of a collapsing bubble. In Fig. 5.6 and 5.7 examples of cell deformation primarily by the viscous mode are captured. Here the cells quickly reach a steady state of small deformation. The membrane is strong compared with the inertial forces and the membrane tension counteracts the tangential viscous stresses caused by the bubble expansion.

The maximum expansion of the contrast agent bubble with respect to the initial volume is representative for the energy stored in the bubble and determines the violence of the subsequent collapse. We have seen that tensile pressures with amplitudes of less than $1 \mathrm{MPa}$ are sufficient to significantly expand the bubble if the duration of the pulse is in the order of several microseconds. Using equation (4.1) one can calculate the maximum expansion of microbubbles depending on pressure amplitude and pulse duration. Figure 5.12 shows the calculated maximum radius of an oscillating microbubble during two sinusoidal pressure excitation periods for frequencies between $100 \mathrm{kHz}$ and $10 \mathrm{MHz}$. The pressure amplitude $P_{e x}$ ranges from $100 \mathrm{kPa}$ to $2 \mathrm{MPa}$ and the initial bubble radius is $R_{0}=1 \mu \mathrm{m}$. All other parameters are the same as in Section 4.1.3. The maximum expansion of bubbles is achieved for high pressure amplitudes at low frequencies. This is to be expected, since bubble growth is governed by the amplitude and duration of the tensile pulse. Significant bubble expansion starts to occur for pressure amplitudes above $\approx 300 \mathrm{kPa}$. Below that threshold, the surface tension is dominant over the exciting pressure. We see that for high frequencies at $\approx 10 \mathrm{MHz}$ the maximum bubble radius remains below $3 \mu \mathrm{m}$ regardless of the pressure amplitude. In this regime we are above the resonant frequency of the bubble, which can be approximated for small amplitude oscillations by [11]:

$$
f_{0}=\frac{1}{2 \pi R_{0}} \sqrt{\frac{3 \gamma}{\rho}\left(P_{0}+\frac{2 \sigma}{R_{0}}\right)-\frac{2 \sigma}{\rho R_{0}}} .
$$

With $R_{0}=1 \mu \mathrm{m}, P_{0}=100 \mathrm{kPa}, \rho=998 \mathrm{~kg} / \mathrm{m}^{3}, \sigma=72.7 \mathrm{mN} / \mathrm{m}$, and $\gamma=1$ we get $f_{0} \approx 4.3 \mathrm{MHz}$. The radius time curves of a microbubble driven at a maximum pressure 


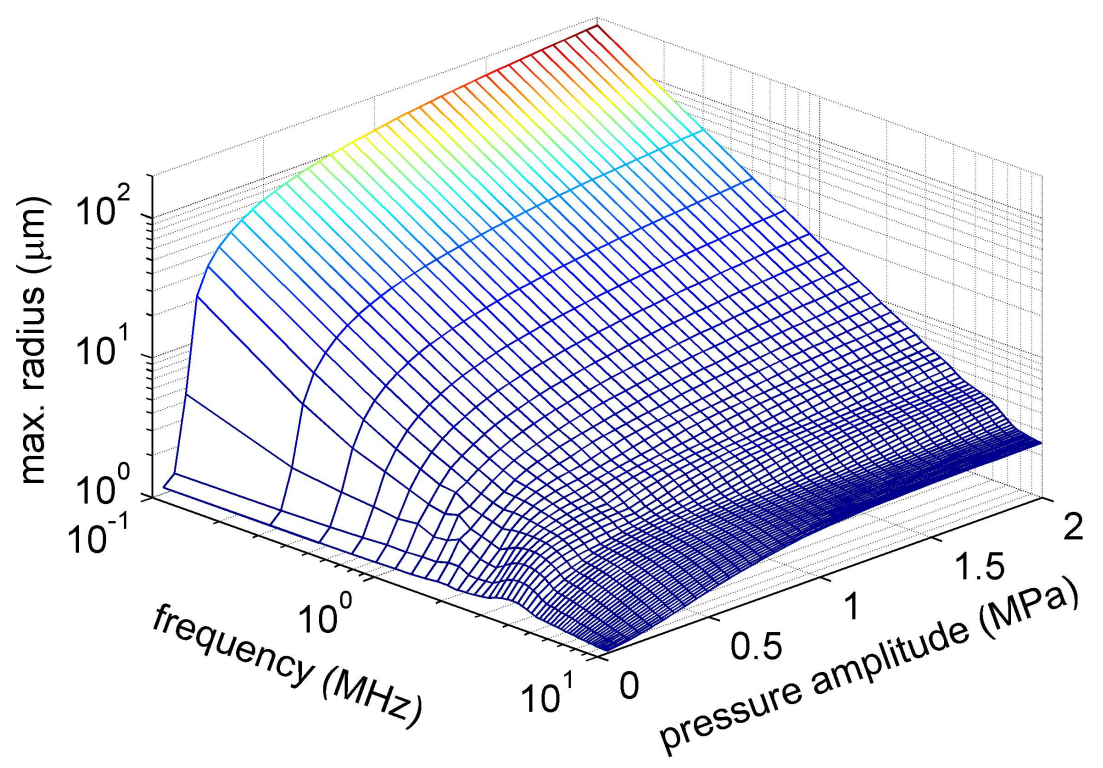

Figure 5.12: Calculated maximum bubble radius during the first two periods of a sinusoidal pressure excitation dependent on frequency and pressure amplitude. The initial radius of the bubble is $1 \mu \mathrm{m}$.

amplitude of $2 \mathrm{MPa}$ below $(f=200 \mathrm{kHz})$ and above $(f=10 \mathrm{MHz})$ the resonance frequency is given in Fig. 5.13. At the high driving frequency the duration of the negative pressure is too short for the bubble to grow substantially in size. After the first tensile period the bubble oscillates with a maximum radius of less than three micrometers and collapses after the third subsequent positive pressure peak. For the low driving frequency the bubble has enough time to expand and accelerate the surrounding liquid during the negative pressure period. Maximum bubble expansion and bubble collapse occur every period but follow the pressure with a significant phase shift due to the inertia of the liquid surrounding the bubble. Below the resonance the maximum radius increases potentially with decreasing frequency as can be seen from the double logarithmic plot in Fig 5.12. As long as the pressure is well above the mentioned threshold, the frequency seems to be the critical parameter for bubble growth in the given range. This is similar to the experimental findings, where we have seen that the low amplitude tensile pulse is more efficient in expanding contrast agent bubbles than the fast high amplitude pressure signal. Figure 5.14 shows a contour plot of maximum bubble diameter depending on frequency and pressure amplitude for the same data as used in Fig 5.12. Since the maximum bubble size is also an indication for collapse strength this plot is a rough estimate of the destructive behavior of excited microbubbles for a given pressure amplitude and frequency. 
(a)

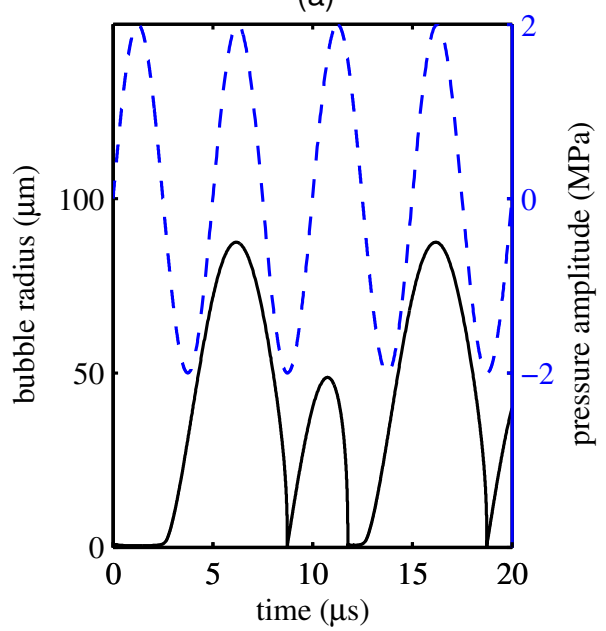

(b)

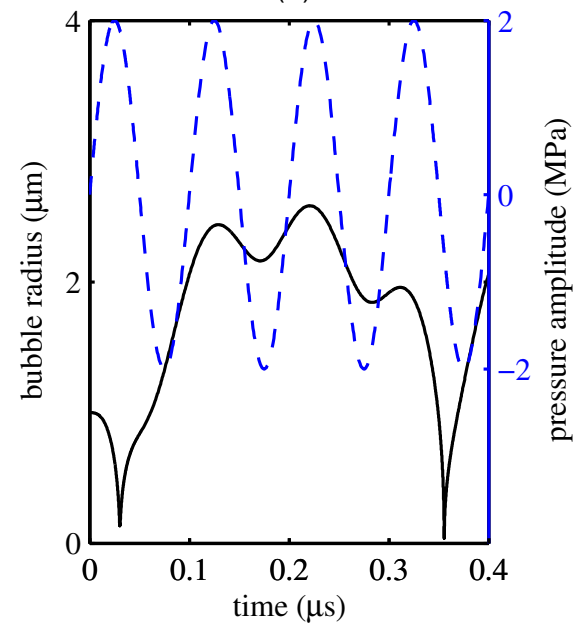

Figure 5.13: Calculated radius time curves of a bubble $\left(R_{0}=1 \mu \mathrm{m}\right)$ during sinusoidal pressure exposure. The pressure is depicted by the dotted blue curves of the figure. Driving frequencies are $200 \mathrm{kHz}$ (a) and $1 \mathrm{MHz}(\mathrm{b})$.

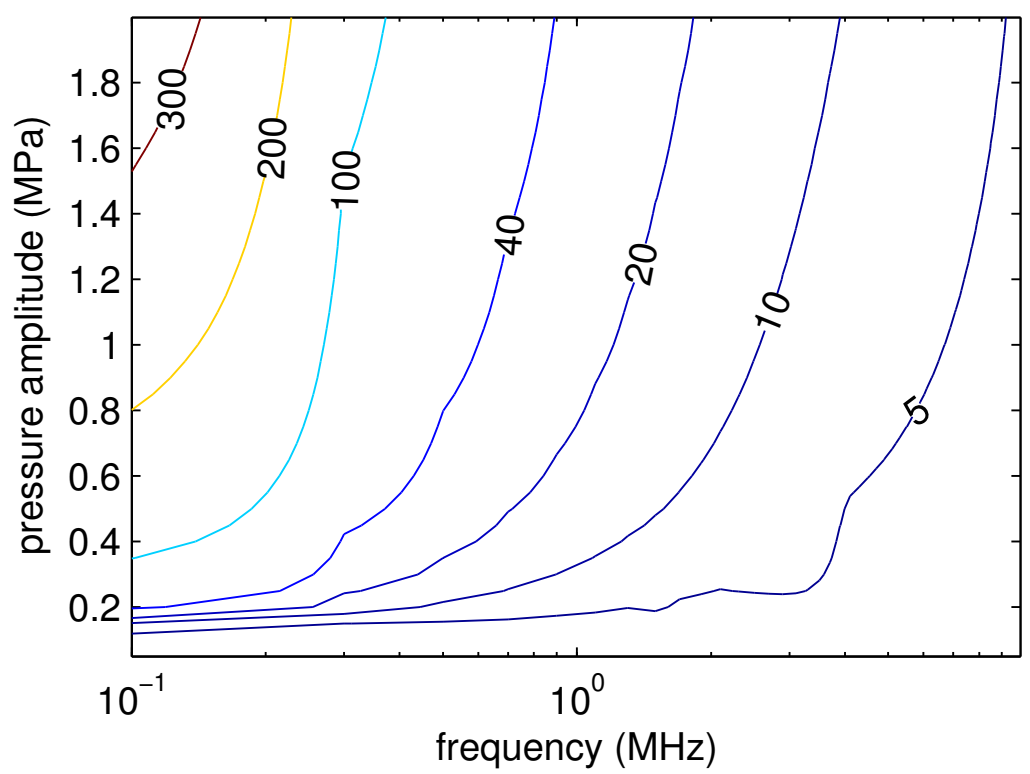

Figure 5.14: Contour plot of the maximum bubble size during sinusoidal pressure excitation depending on frequency and pressure amplitude. The numbers refer to the maximum bubble diameter during the first two pressure periods in micrometers. The initial bubble diameter is $2 \mu \mathrm{m}$. 


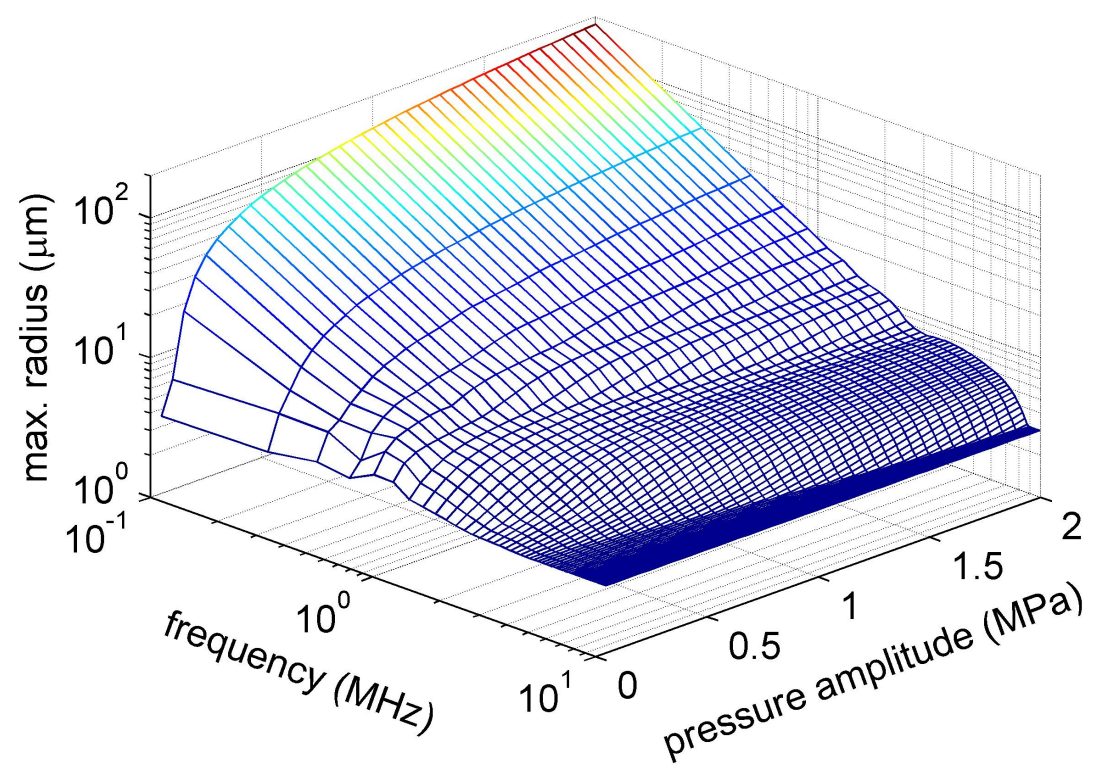

Figure 5.15: Calculated maximum bubble radius during the first two periods of a sinusoidal pressure excitation dependent on frequency and pressure amplitude. The initial radius of the bubble is $3 \mu \mathrm{m}$.

In the experiments expanded contrast agent bubbles of $40 \mu \mathrm{m}$ diameter were observed to visibly damage cells upon collapse. With Fig. 5.14 it is possible to separate the parameter space into regions above and below a threshold believed to be potentially hazardous for cells. However, care has to be taken when applying the calculations to the expansion of coated microbubbles in a confined geometry. Boundaries and coating will decrease the maximum expansion of bubbles rendering the calculations an upper bound for the bubble size. Also it should be noted that the maximum expansion is not the only criterion for collapse strength and cell damage. Other factors such as cell and bubble density or direct interactions of acoustic waves and cells should additionally be considered when assessing the potential for cell damage of a certain application [131].

In Fig. 5.15 and 5.16 the maximum bubble expansion for a microbubble with initial radius $R_{0}=3 \mu \mathrm{m}$ is depicted. Comparing the plots with Fig. 5.12 and 5.14 we can see that the initial size changes little to maximum bubble expansion. The resonance frequency is somewhat lower for larger bubbles, which follows directly from equation (5.1). Below that resonance, the maximum bubble radius increases towards decreasing frequencies obtaining only slightly larger values than the bubbles with smaller initial size $\left(R_{0}=1 \mu \mathrm{m}\right)$. Already at a maximum diameter of $40 \mu \mathrm{m}$ the contour lines dividing the parameter space are 


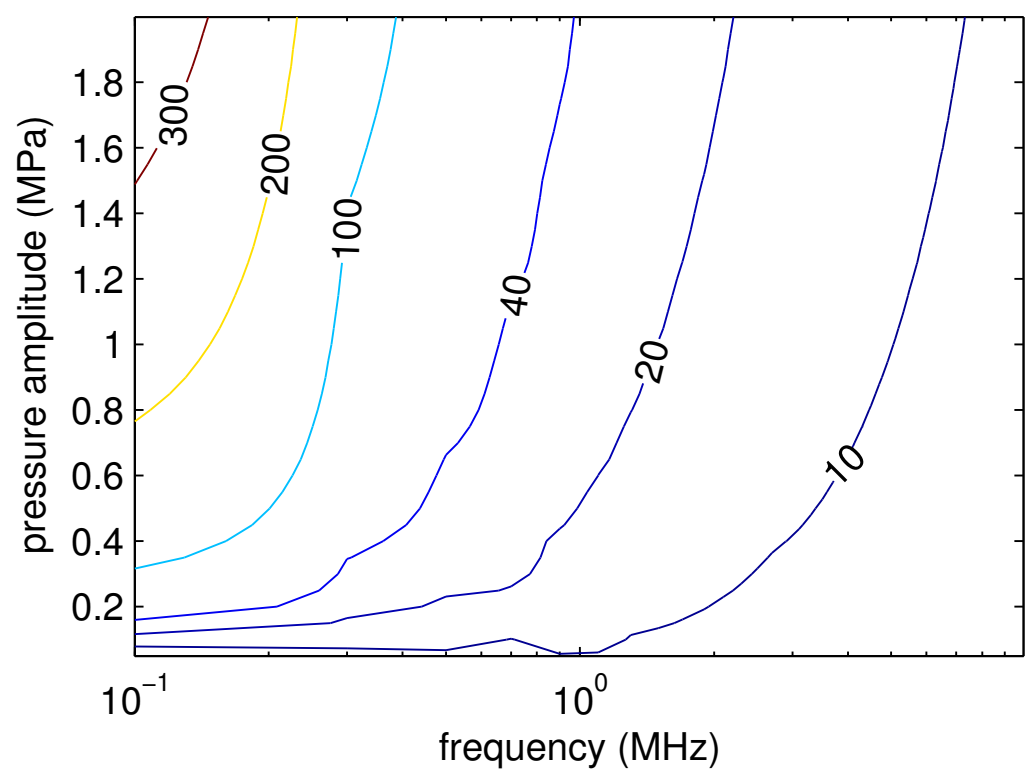

Figure 5.16: Contour plot of the maximum bubble size during sinusoidal pressure excitation depending on frequency and pressure amplitude. The numbers refer to the maximum bubble diameter during the first two pressure periods in micrometers. The initial bubble diameter is $6 \mu \mathrm{m}$.

approximately the same in Fig. 5.14 and Fig. 5.16 .

\subsection{Conclusion}

In this chapter, the effects of pressure excited contrast agent bubbles on cells in vitro were observed with high-speed microscopic techniques. Even low tensile pressure amplitudes of less than $\approx 1 \mathrm{MPa}$ are sufficient to cause strong expansion of initially micron sized contrast agents. The bubbles may grow to achieve radii of more than $30 \mu \mathrm{m}$, thereby greatly surpassing the size of the cells. A critical parameter for the maximum expansion is the duration of the negative pressures cycle. Subsequent collapse of largely expanded bubbles may cause visible damage to the cells. From the basis provided by these experiments further work should be directed at combining high-speed optical recordings with biochemical techniques to assess the viability and permeabilization of cells exposed to a collapsing microbubble in its vicinity. 


\section{Chapter 6}

\section{Shock wave and cavitation generation after impact of high-speed projectiles on tissue substitutes}

In the two preceding experiments described in Chapters 4 and 5 the focus was placed on pressure waves and cavitation activity in microscopic systems. Before delving further into the interactions of cavitation bubbles with culture cells in Chapter 7, some examples of shock wave and cavitation generation in macroscopic systems following the impact of high-speed projectiles will be presented. In the experiments, water or highly aqueous substances like gelatin or melons have been used as targets to simulate bullet impact on tissues. The processes during ballistic injuries are especially important to forensic doctors who try to recapitulate the scenario of injury by gathering evidence from the victim. In here, high-speed imaging techniques are used to capture processes during bullet impact. The main emphasis is thereby placed on the generation of shock waves and cavitation and their dependence on the speed of the projectile. The experiments were performed in cooperation with the forensic department of the University of Göttingen [132].

\subsection{Experimental setup}

The experimental setup is depicted in Fig. 6.1. The target is placed either on a wooden board or a block of gelatin. An Imacon high-speed camera described in Chapter 4 with a $50 \mathrm{~mm}$ or $28 \mathrm{~mm}$ objective is used to take images during bullet impact. The scene is illuminated by a $600 \mathrm{~W}$ continuous wave lamp. For some trials the pressure is recorded with a fiber-optic hydrophone also described in Chapter 4. Before entering the target, the 


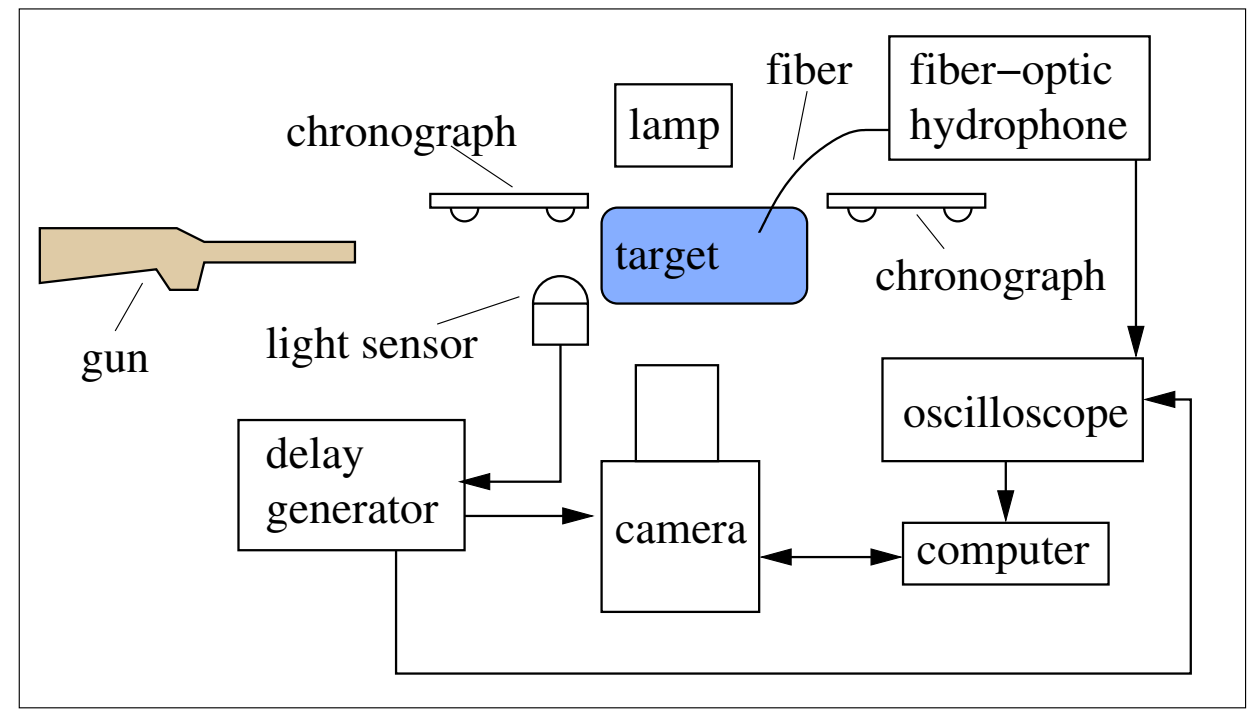

Figure 6.1: Setup for observation and pressure measurements during bullet impact on aqueous targets.

projectile triggers a light sensor and its speed is measured with a chronograph (Chrony M-2 Delta, Shooting Chrony). A second chronograph is located behind the target to measure the speed of the bullet in case it is able to penetrate the target. The trigger delay times for camera and hydrophone recordings are estimated from the bullet speed and the distance of the light sensor to the target. The projectiles used are mainly full metal jackets $5.67 \mathrm{~mm}$ in diameter and $53.5 \mathrm{~mm}$ in length weighing 3 grams. To achieve bullets with velocities of more than $1000 \mathrm{~m} / \mathrm{s}$ a pellet of 1.35 grams of gun powder (N110, Vitavuori) or 1.66 grams of N130 (Vitavuori) has been added into the socket in front of the blasting cap. To achieve lower bullet speeds $(\approx 770 \mathrm{~m} / \mathrm{s}) 1$ gram of N120 (Vitavuori) has been used, see [133] for details on the gun powder and bullet types. In the following experiments projectiles traveling at a speed of more than $1000 \mathrm{~m} / \mathrm{s}$ will be called highspeed projectiles.

Due to the violent nature of the experiment the pressure recordings are problematic in this setup. Typically the target explodes after bullet impact, which increases the risk of damage to the pressure probe. From this point of view a fiber-optic hydrophone is a good choice as a pressure measurement device, since the broken fiber can be reused after cutting. However, a problem arises when the end of the fiber is exposed to air after the target is destroyed. In this case the reflection of the laser light at the fiber tip becomes very large because of the difference between refraction indices of the fiber core and air. The reflected laser light may then damage the internal photodiode of the hydrophone. 
Kap. 6 Shock wave and cavitation generation after impact of high-speed projectiles

on tissue substitutes

It is therefore necessary to operate the hydrophone laser at a small power amplitude, resulting in a decreased pressure resolution of about $10 \mathrm{MPa}$.

\subsection{Impact of high-speed bullets on aqueous substances and secondary effects}

Figure 6.2 shows impressively the difference between the impact of a "normal" $(772 \mathrm{~m} / \mathrm{s})$ and a high-speed $(1063 \mathrm{~m} / \mathrm{s})$ projectile in a melon. The slower bullet shown in Fig. 6.2 (a) penetrates the fruit causing a backward splash at the entry side and a somewhat larger splash on the exit side of the melon. However, the overall shape of the melon stays intact after the bullet has penetrated the melon. Behind the target the bullet speed was measured to be $703 \mathrm{~m} / \mathrm{s}$. The energy transferred to the target is approximately equal to the difference in kinetic energies before and after melon penetration, $E_{t}=E_{k i n 0}-E_{k i n 1}$, if energies for deformation, rotation or thermal heating of the bullet are assumed to be small. Since the mass of the projectile was conserved, i.e. the bullet did not fragment, we get

$$
E_{t} \approx \frac{1}{2} m\left(v_{0}^{2}-v_{1}^{2}\right)=153 \mathrm{~J}
$$

which is $\approx 17 \%$ of the initial kinetic energy $\left(E_{k i n 0}=894 \mathrm{~J}\right)$.

After the impact of a high-speed bullet shown in Fig. 6.2 (b) the bullet fragments inside the melon making it impossible to assess the remaining kinetic energy. The melon does not maintain its shape but explodes and fragments into mainly subcentimeter pieces. It is clearly visible that a high-speed impact is much more violent transferring more of the projectile's energy to its target. In the following sections the impact of high-speed bullets on gelatin and water and the resulting shock wave and secondary effects are discussed.

\subsubsection{High-speed bullet impact on gelatin and water}

Gelatin is an often used medium to simulate tissue characteristics [134, 135, 136, 137] in ballistic experiments. It has the advantage of being largely transparent allowing to view processes during bullet penetration. Figures 6.3 and 6.4 show the impact of high-speed bullets in gelatin blocks ( $90 \%$ water content) with different timings.

In Fig 6.3 (a) we see that the bullet advances about $35 \mathrm{~mm}$ in the first $60 \mu \mathrm{s}$. During that time a narrow channel of approximately $12 \mathrm{~mm}$ width is formed in the medium 
(a)

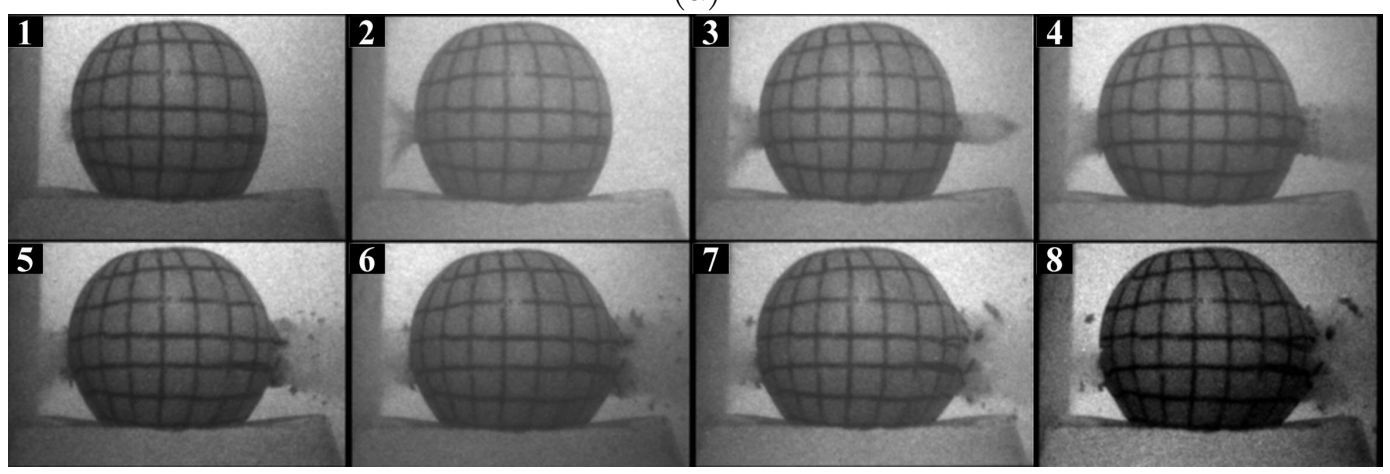

(b)

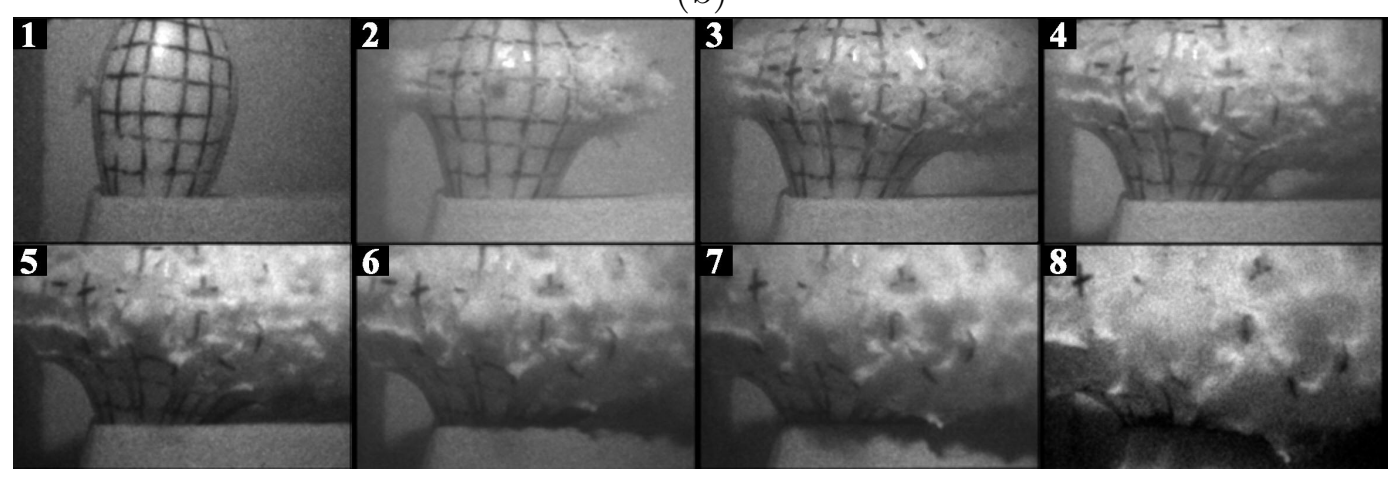

Figure 6.2: Impact of a full metal jacket on melon targets. The bullet speeds before impact are $772 \mathrm{~m} / \mathrm{s}$ for sequence (a) and $1063 \mathrm{~m} / \mathrm{s}$ for sequence (b) of the figure. Interframe times in sequence (a) are $100 \mu$ s from the first to the fourth frame and $200 \mu \mathrm{s}$ after frame 4 . In sequence (b) the interframe times are $300 \mu \mathrm{s}$ after the first frame, $200 \mu$ s between the second and seventh frame, and $400 \mu \mathrm{s}$ between frame 7 and 8 . Exposure times are $10 \mu \mathrm{s}$ for both image sequences. The size of a single frame is $227 \times 152 \mathrm{~mm}^{2}$. 
Kap. 6 Shock wave and cavitation generation after impact of high-speed projectiles

(a)

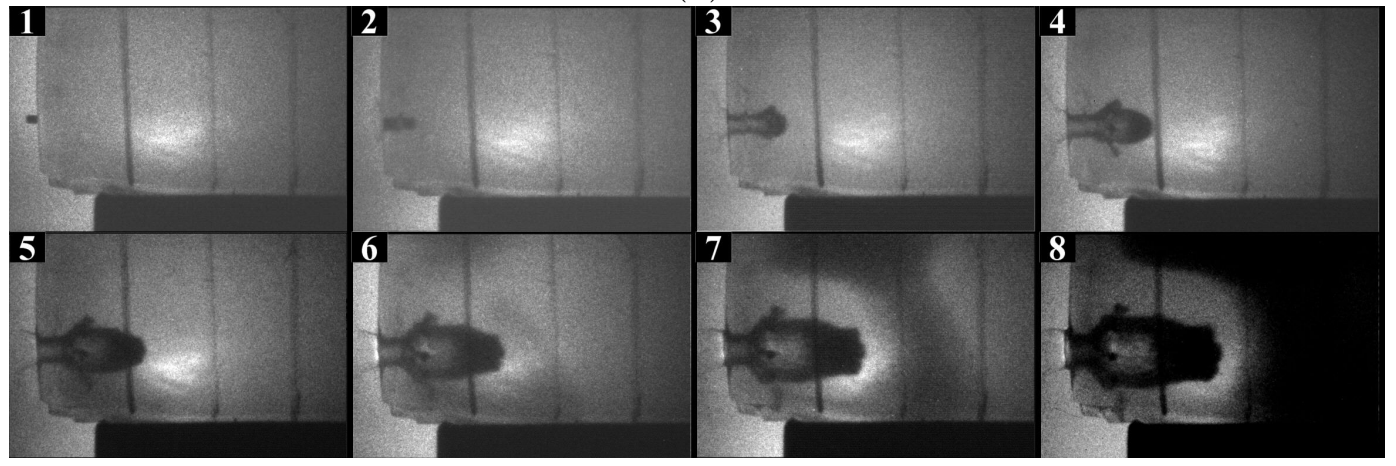

(b)

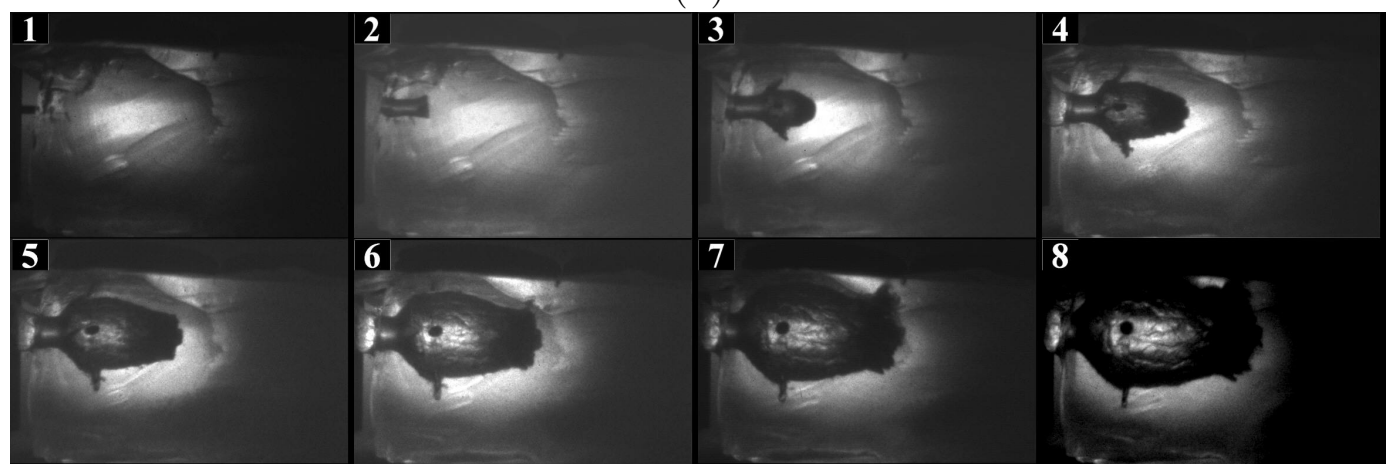

Figure 6.3: Impact of a high-speed projectiles in gelatin. The exposure time for each frame is $2 \mu \mathrm{s}$. In sequence (a) the interframe times are $22 \mu \mathrm{s}$ and the bullet speed before impact is $1083 \mathrm{~m} / \mathrm{s}$. In sequence (b) the interframe times are $35 \mu \mathrm{s}$ and the bullet speed before impact is $\approx 1070 \mathrm{~m} / \mathrm{s}$. The size of a single frame is $227 \times 152 \mathrm{~mm}^{2}$. 
(a)

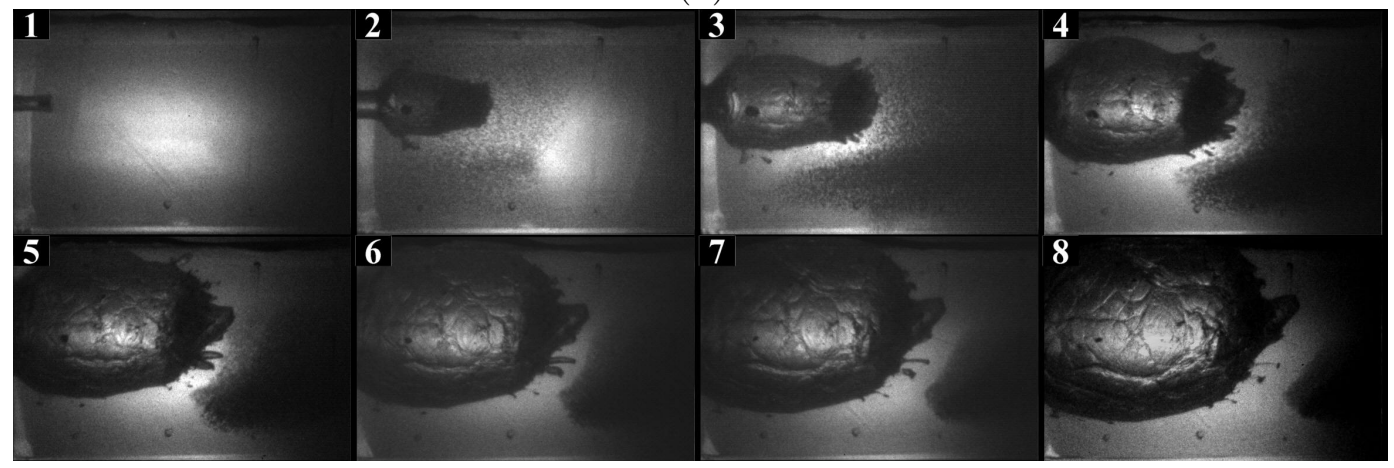

(b)

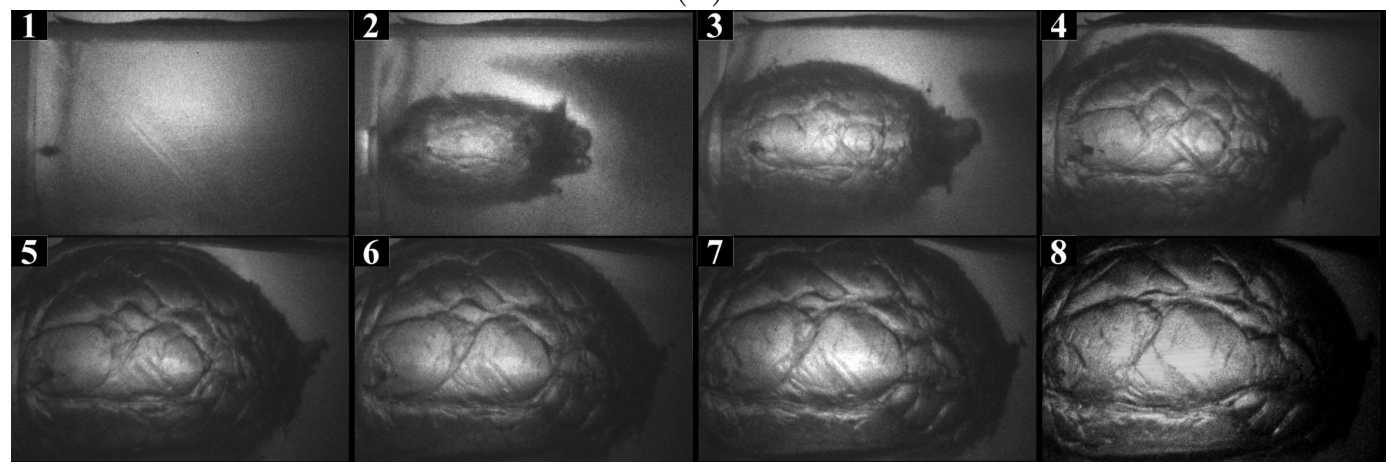

Figure 6.4: Impact of a high-speed projectiles in gelatin. The exposure time for each frame is $2 \mu \mathrm{s}$. In sequence (a) the interframe times are $100 \mu \mathrm{s}$. In sequence (b) the interframe times are $300 \mu \mathrm{s}$. For both shots the bullet speed before impact is $1069 \mathrm{~m} / \mathrm{s}$. The size of a single frame is $227 \times 152 \mathrm{~mm}^{2}$. 
Kap. 6 Shock wave and cavitation generation after impact of high-speed projectiles

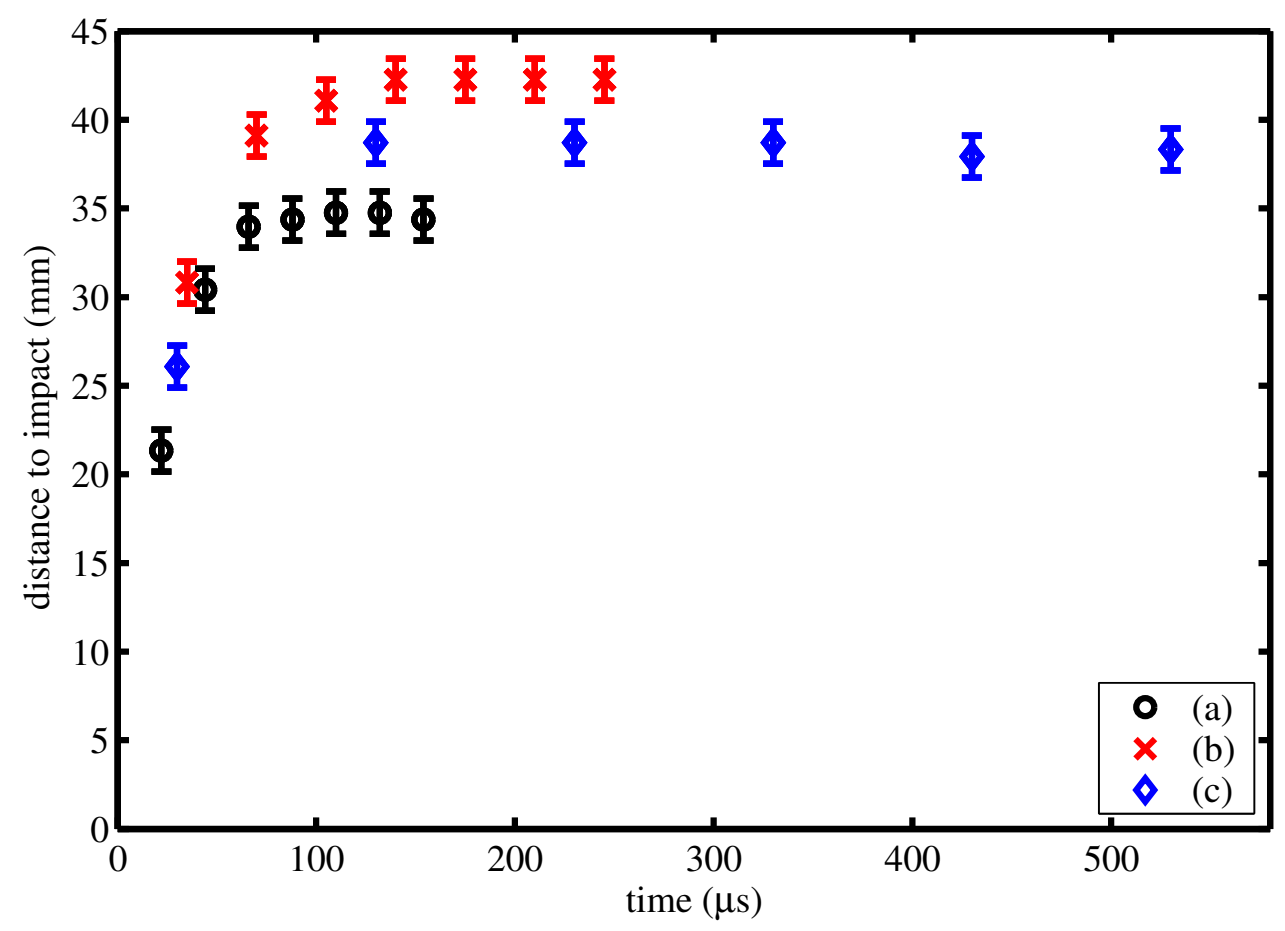

Figure 6.5: Distance of bullet to impact location versus time for three different shots; (a): sequence (a) of Fig. 6.3, (b): sequence (b) of Fig. 6.3, (c): sequence (a) of Fig. 6.4. The time is given with respect to the approximate impact of the projectile on the medium.

following the projectile's path. Unlike slower projectiles, which can penetrate a $40 \mathrm{~cm}$ block of gelatin fairly unimpeded, almost all the high-speed bullet's momentum is lost after advancing a few centimeters into the medium. Figure 6.5 shows the position of the projectile with respect to the impact location depending on time for sequence (a) and (b) of Fig 6.3 and sequence (a) of Fig 6.4. We see that all three bullets stop after penetrating 34 to $43 \mathrm{~mm}$ into the medium.

The kinetic energy of a high-speed projectile with a speed of $1100 \mathrm{~m} / \mathrm{s}$ before impact is $E_{k i n 0}=1815 \mathrm{~J}$. After impact some of this energy is used to deform or fragment the bullet, while other parts are transferred to the surrounding medium leading to the formation of a large cavity and the emission of pressure waves. In frame 8 of Fig. 6.4 (b) it is shown that approximately $2.1 \mathrm{~ms}$ after bullet impact the vertical diameter of the cavity already surpasses the initial height of the gelatin block $(15 \mathrm{~cm})$. Sometimes the large deformation leads to a rupture of the gelatin block. However, because of the high elasticity of the medium, the reset force usually closes the primary cavity before breakage of the block can 


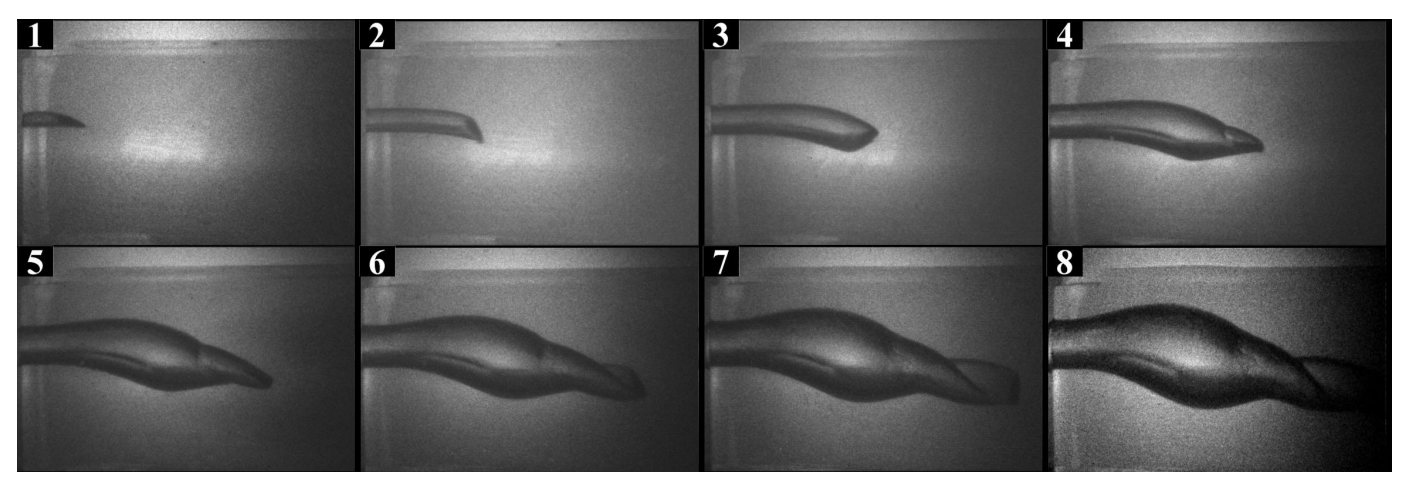

Figure 6.6: Impact of a solid high-speed projectile in gelatin. The bullet speed before impact is $1044 \mathrm{~m} / \mathrm{s}$. Exposure and interframe times are $2 \mu \mathrm{s}$ and $42 \mu \mathrm{s}$, respectively. The size of a single frame is $227 \times 152 \mathrm{~mm}^{2}$.

occur. In this case, subsequent analysis of the block only shows minor signs of damage due to the bullet fragments and some cuts caused by the primary cavity.

In the sequences of Figs. 6.3 and 6.4 we can see a cloud of dark spots appearing approximately $100 \mu$ s after bullet impact. The cloud consists of cavitation bubbles. They are created when the negative pressure, following the reflection of the shock wave at the edges of the gelatin block, drops sufficiently low to rupture the gelatin matrix. The bubble cloud therefore emerges at the edges of the gelatin block and follows the reflected pressure wave into the medium. In the regions directly adjacent to the primary cavity the cavitation cloud disappears quickly. This suggests that a positive pressure in front of the boundary is responsible for rapid recompression of the bubbles.

The large transfer of kinetic energy from the high-speed projectile into the medium is most probably connected to the deformation of the bullet upon impact. It has been known for a long time that large bullet wounds also appear when readily deformable dumdum projectiles are used at lower speeds 1 . Normal-speed or robust high-speed projectiles on the other hand do not display the typical short penetration distance and violent explosion observed after impact of high-speed metal jackets. For example in Fig. 6.6 we see a solid high-speed bullet milled out of brass penetrate a gelatin block without fragmentation. Having a velocity of $1044 \mathrm{~m} / \mathrm{s}$ the solid brass projectile is only slightly slower than the full metal jackets observed in Figs. 6.3 and 6.4. The solid projectile tumbles after impact but still penetrates the whole block of gelatin remaining largely undeformed. In this

\footnotetext{
${ }^{1}$ The resulting severe injuries led to the banning of easily flattening or expanding bullets in the 1899 Hague Declaration.
} 
Kap. 6 Shock wave and cavitation generation after impact of high-speed projectiles 80

on tissue substitutes

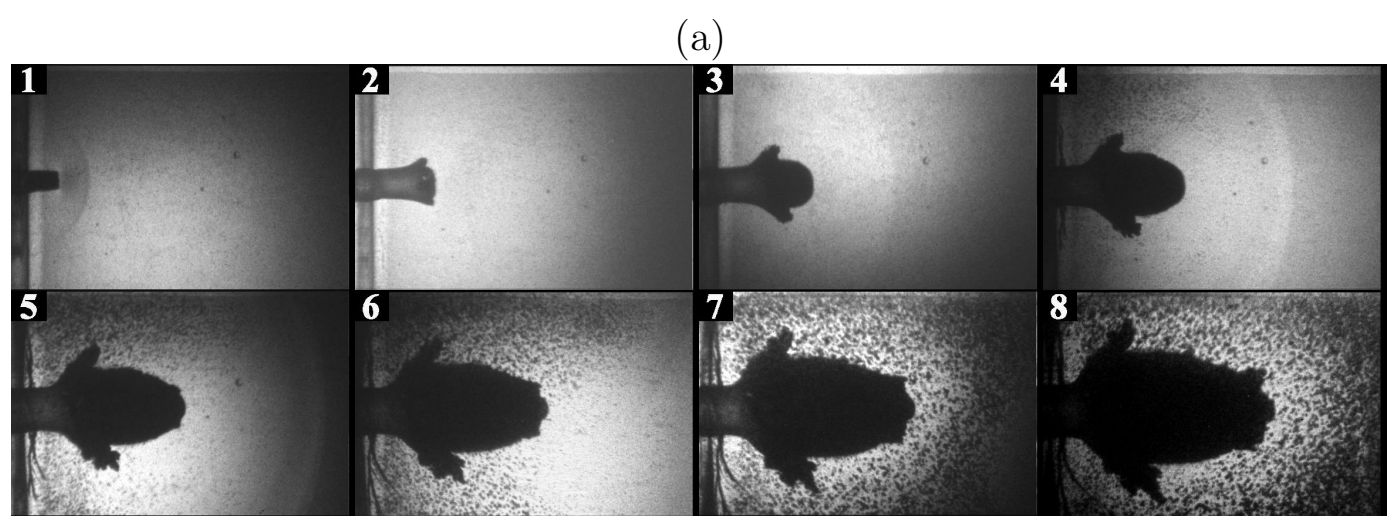

(b)

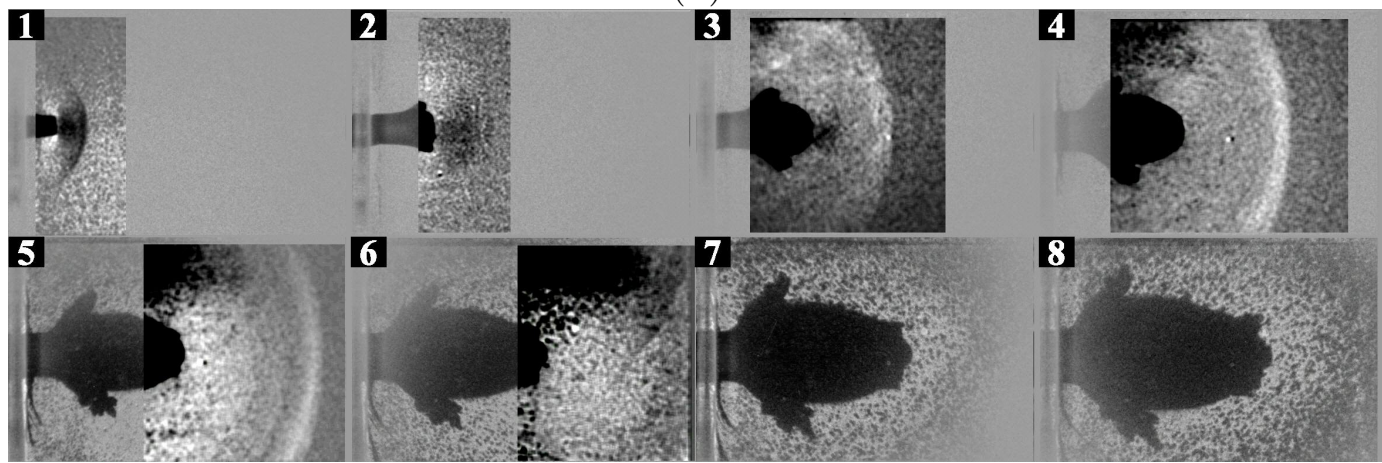

Figure 6.7: Impact of a high-speed projectile $(1132 \mathrm{~m} / \mathrm{s})$ in water. Part (b) shows the background corrected frames of (a) with partly enhanced contrast to better visualize the shock wave. Exposure and interframe times are $1 \mu$ s and $15 \mu \mathrm{s}$, respectively. The size of a single frame is $137 \times 92 \mathrm{~mm}^{2}$.

experiment no cavitation bubbles are observed. This indicates that the negative amplitude of the reflected shock wave is smaller than during impact of full metal jackets at similar speeds.

Using water as a target, cavitation and shock wave effects are better visible than in gelatin. Figures 6.7 and 6.8 show the impacts of high-speed bullets in water with different interframe times and container sizes. The water is contained in rectangular plastic vessels with a wall thickness of $1 \mathrm{~mm}$. Part (b) of both figures shows the background corrected frames with partly enhanced contrast to highlight the shock waves.

In frames 1, 3, 4, and 5 of Fig. 6.7 (b) we see the outgoing shock wave after bullet impact. For a bullet of $1125 \mathrm{~m} / \mathrm{s}$ initial speed the maximum pressure amplitude of the shock has been measured to be approximately $110 \mathrm{MPa}$ at a distance of $16.5 \mathrm{~mm}$ to the target entry site about $10 \mathrm{~mm}$ away from the axis of bullet propagation. The initial pressure 
(a)

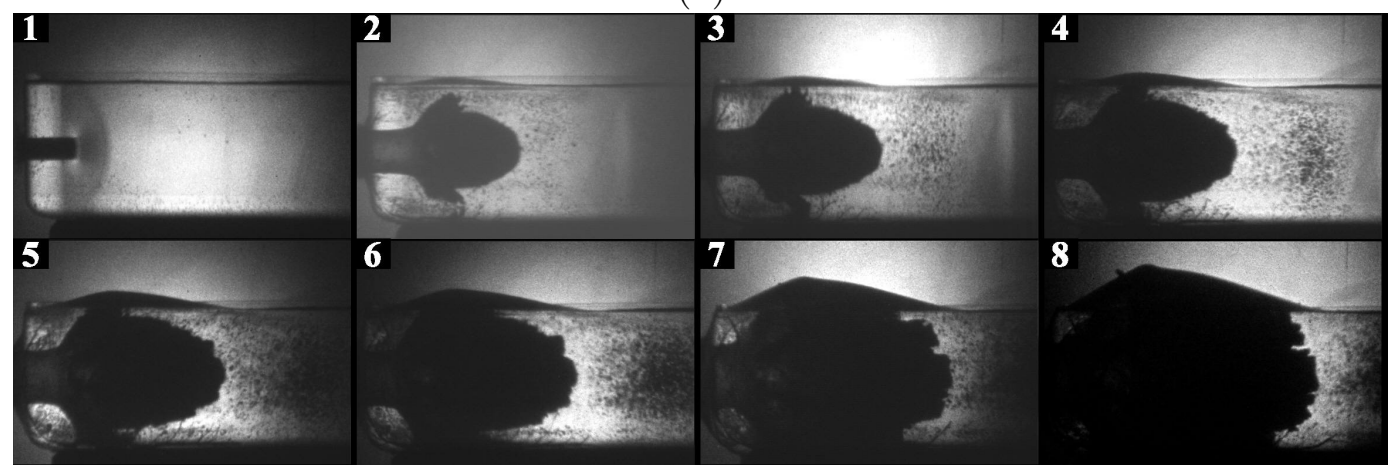

(b)

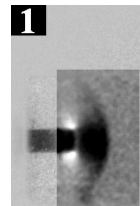

5

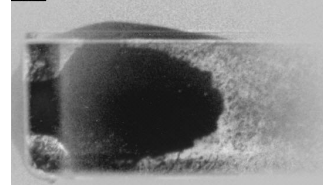

2

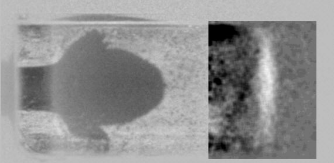

6

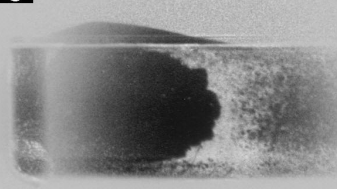

3

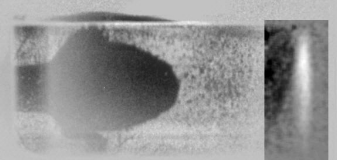

7

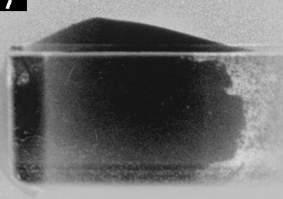

4

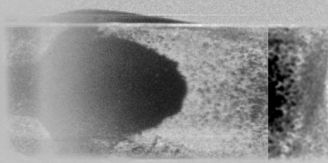

8

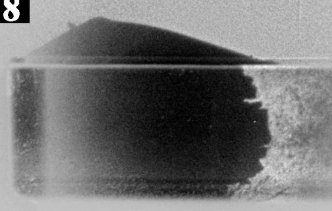

Figure 6.8: Impact of a high-speed projectile $(1125 \mathrm{~m} / \mathrm{s})$ in water. Part (b) shows the background corrected frames of (a) with partly enhanced contrast to better visualize the shock wave. The exposure time is $2 \mu \mathrm{s}$. Interframe times are $45 \mu$ s after the first frame, $10 \mu$ s from frame 2 to frame 6 and $25 \mu$ s after the sixth frame. The size of a single frame is $137 \times 92 \mathrm{~mm}^{2}$. 
Kap. 6 Shock wave and cavitation generation after impact of high-speed projectiles

upon bullet impact can be approximated in several ways. A method independent of shock wave amplitude or shock wave velocity measurements is given by the water hammer equation [138]

$$
P_{w h}=\frac{c_{1} \rho_{1} c_{2} \rho_{2}}{c_{1} \rho_{1}+c_{2} \rho_{2}} v_{b}
$$

Equation (6.1) can be used to describe the impact of a brass stream on water with densities $\rho_{1}=8500 \mathrm{~kg} / \mathrm{m}^{3}, \rho_{2}=998 \mathrm{~kg} / \mathrm{m}^{3}$ and sound velocities $c_{1}=3500 \mathrm{~m} / \mathrm{s}, c_{2}=1500 \mathrm{~m} / \mathrm{s}$ for brass and water, respectively. The resulting pressure for a bullet impact speed of $v_{b}=1125 \mathrm{~m} / \mathrm{s}$ is $P_{w h}=1603 \mathrm{MPa}$. If instead of brass a lead stream is used to describe a full metal jacket impact on water, the corresponding pressure amounts to $1537 \mathrm{MPa}$ for $\rho_{\text {lead }}=13000 \mathrm{~kg} / \mathrm{m}^{3}$ and $c_{\text {lead }}=1200 \mathrm{~m} / \mathrm{s}$. It should be noted that equation (6.1) is derived from a 1-dimensional setting and therefore neglects any geometrical effects. Also the variation in the speed of sound propagation with pressure is not taken into account. The latter leads to an underestimation of the pressure, since the speed of sound or shock wave propagation increases with pressure.

We can also deduct the initial shock wave strength from the hydrophone measurement (110 MPa at a distance of $16.5 \mathrm{~mm}$ to the location of target entry). In the acoustic approximation of a purely geometrical approach the pressure $P(r)$ at an arbitrary distance $r$ is given by

$$
P(r)=P_{i n} r_{i n} / r
$$

Thereby it is assumed that the initial pressure $P_{i n}$ is generated from a spherical source of radius $r_{i n}$ and dissipative effects at the shock front are neglected. At an initial radius of $r_{i n}<2.78 \mathrm{~mm}$, which conforms of the width of the projectile, the resulting initial pressure is $P_{\text {in }}>653 \mathrm{MPa}$.

The resulting initial pressure has to be seen as lower bound for the following reasons. Firstly, dissipative processes due to thermal conduction and viscosity at the shock front were neglected. Secondly, the projectile is initially pointed and the pressure source will therefore have a smaller initial radius. For example, assuming an initial radius of $1 \mathrm{~mm}$ one gets an initial pressure of $P_{i n}=1815 \mathrm{MPa}$.

Experimental results from the detonation of TNT under water show that the peak pressure of the shock wave decreases with $P(r) \sim 1 / r^{1.13}$ for pressure values between 4 and $170 \mathrm{MPa}$ [64]. Figure (6.9) shows a semilogarithmic plot of the peak pressure against the radius for the empirical $\left(P(r) \sim 1 / r^{1.13}\right)$ and the acoustic $(P(r) \sim 1 / r)$ approximation, with the pressure measurement providing a point of reference. The upper and lower curves take into account the uncertainty of the location of the pressure probe. 


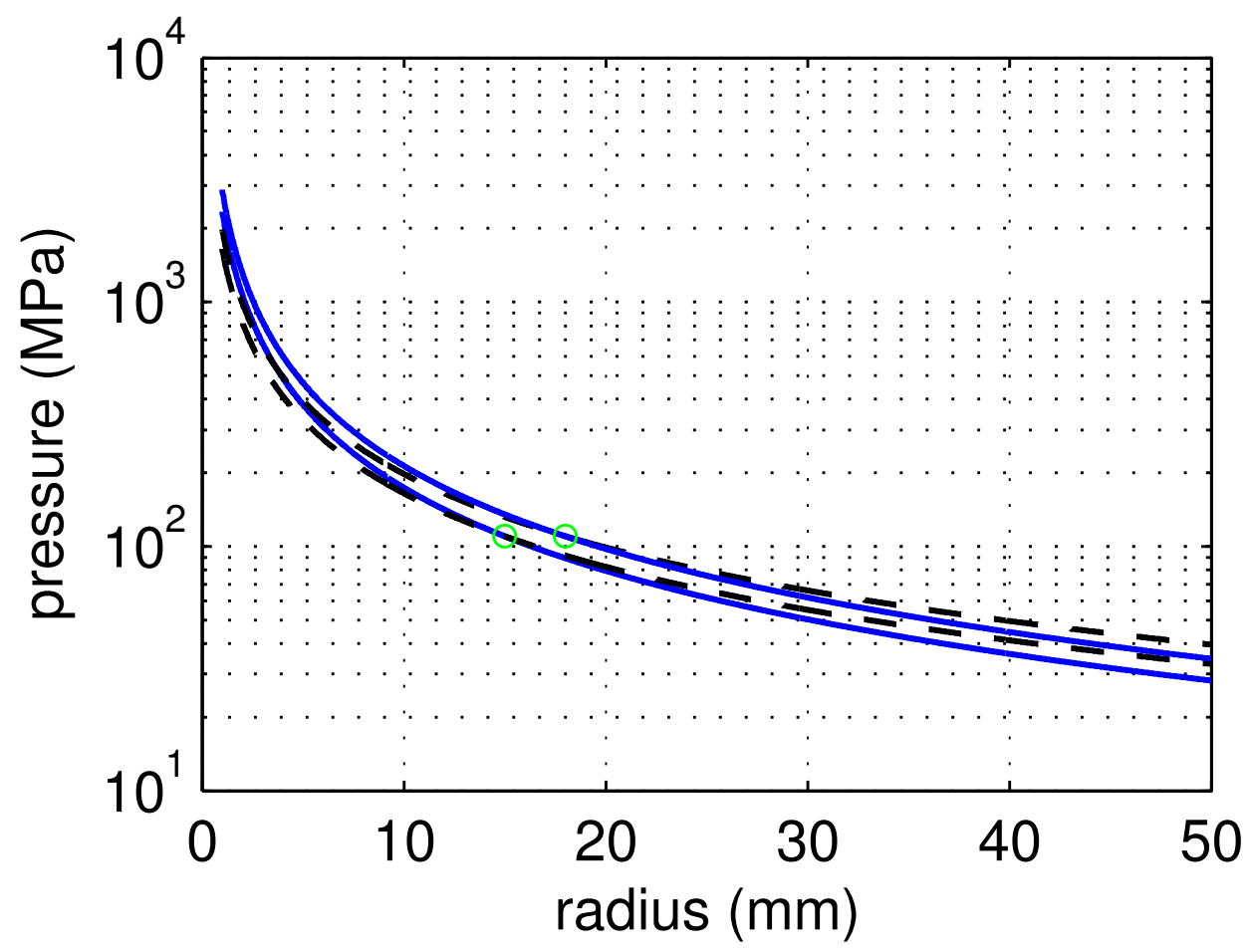

Figure 6.9: Semilogarithmic plot of the pressure versus radius for the propagation of a spherical shock wave after bullet impact. A pressure of $110 \mathrm{MPa}$ at a distance of 15 and $18 \mathrm{~mm}$ to the impact location has been used as a reference (green circles). The blue curves display the empirical relation $P(r) \sim 1 / r^{1.13}$ derived from pressure measurements after the detonation of TNT charges under water [64], while the black dashed curves represent the acoustic approximation $P(r) \sim 1 / r$. Upper and lower curves account for uncertainty in the location of the pressure probe.

For the initial pressure at $r_{i n}=2.78 \mathrm{~mm}$ the empirical relation gives us a value between 740 and $910 \mathrm{MPa}$, which is somewhat higher than the result obtained from the acoustic approximation (see Fig. 6.10). However, it should be noted that these values are well above the range of experimental verification, for which the empirical relation was obtained. It is therefore to be expected, that even using this relation the pressure is underestimated, since strong initial dissipation is not accounted for. Calculating the pressures with smaller initial radii leads to very large pressures, which however decrease rapidly due to geometrical spreading and dissipation.

A further approximation of the initial pressure independent of the direct pressure mea- 
Kap. 6 Shock wave and cavitation generation after impact of high-speed projectiles

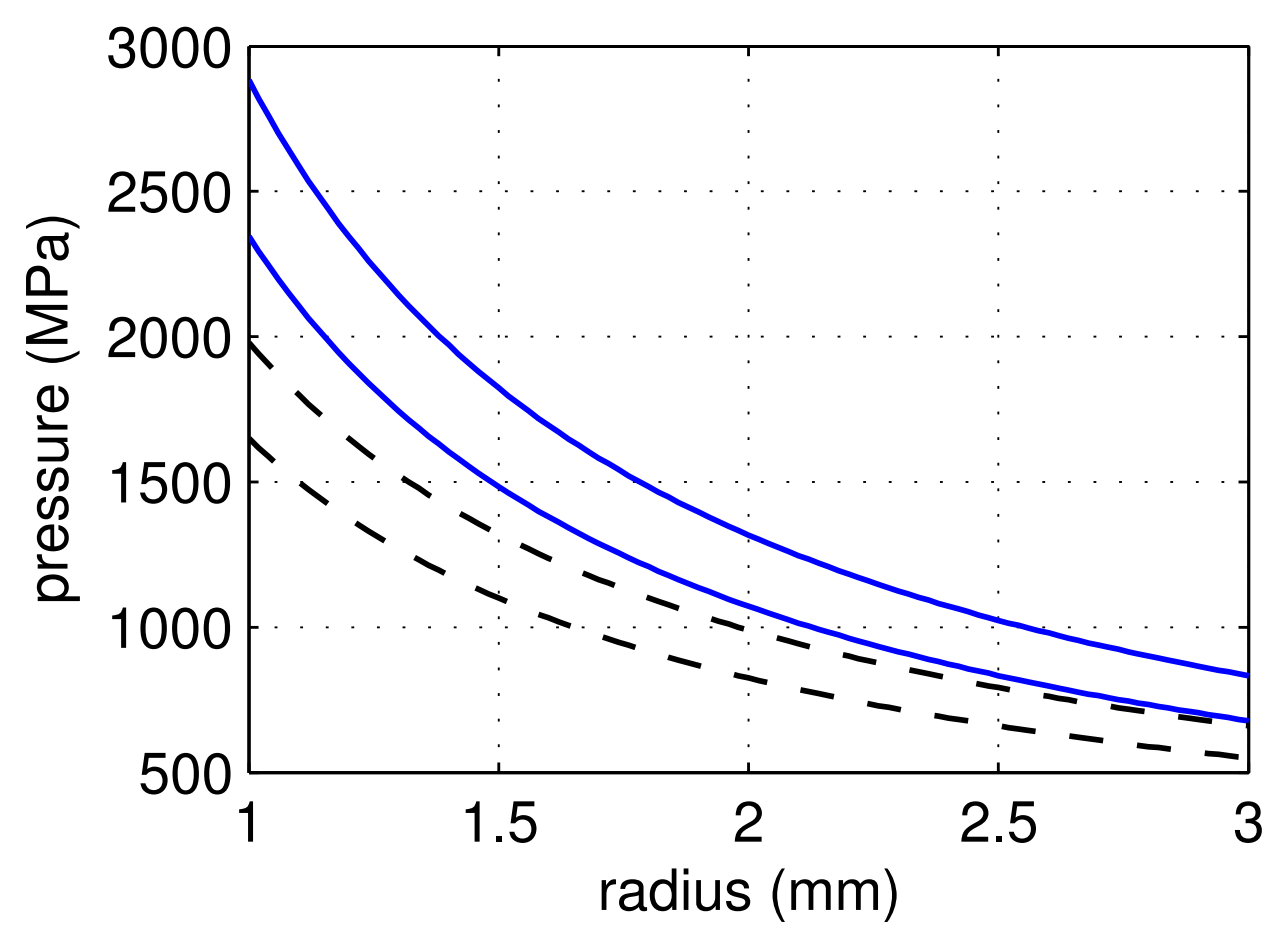

Figure 6.10: Initial pressure dependent on the initial radius for a spherical shock wave after bullet impact. A pressure of $110 \mathrm{MPa}$ at a distance of 15 and $18 \mathrm{~mm}$ to the impact location has been used as a reference. The blue curves display the empirical relation $P(r) \sim 1 / r^{1.13}$ derived from pressure measurements after the detonation of TNT charges under water [64], while the black dashed curves represent the acoustic approximation $P(r) \sim 1 / r$. Upper and lower curves account for uncertainty in the location of the pressure probe. 


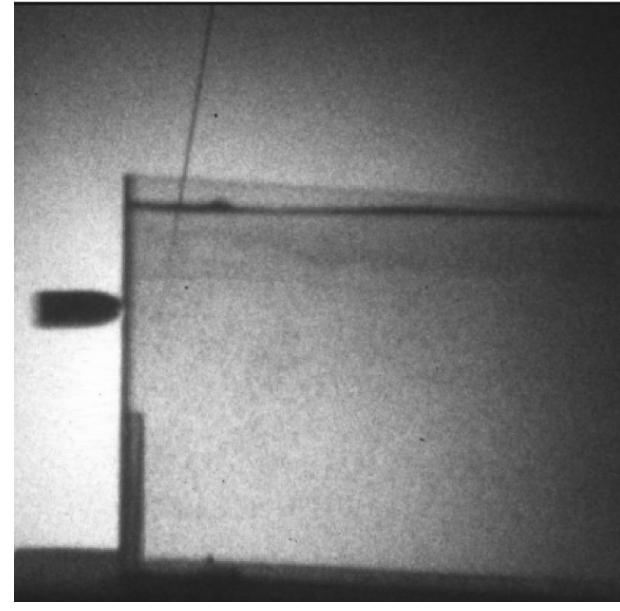

(a)

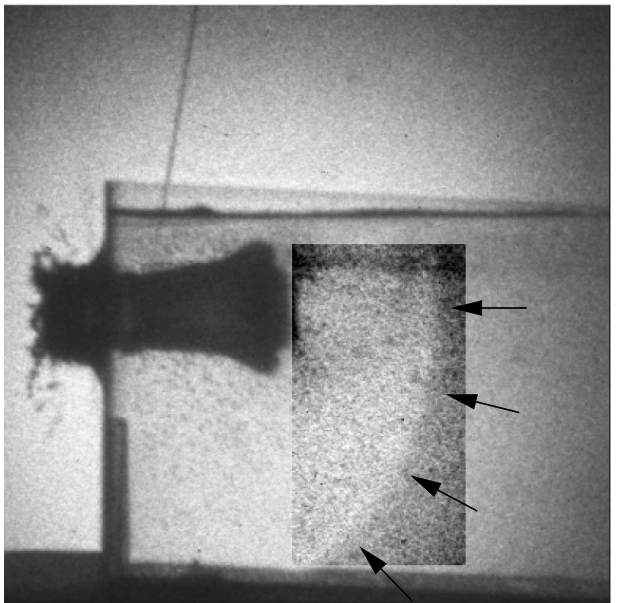

(b)

Figure 6.11: Water filled vessel just before bullet impact (a) and $30 \mu$ s later (b). In part (b) the position of the shock wave is marked by arrows. The bullet speed before impact is $1125 \mathrm{~m} / \mathrm{s}$. Exposure times are $2 \mu \mathrm{s}$. The size of a single frame is $92 \times 91 \mathrm{~mm}^{2}$.

surement can be obtained from the shock wave position in the image data of Fig 6.11. From equation (3.9) we get a relationship between the local pressure $P(r)$ and the local shock wave velocity $U(r)$ :

$$
U(r)=\sqrt{\frac{P(r)-P_{0}}{\rho_{0}-\rho_{0}\left(\frac{P(r)+B}{P_{0}+B}\right)^{-1 / n}}} .
$$

Integrating over the reciprocal shock velocity yields the propagation time $T$

$$
d r=U(r) d t \quad \rightarrow \quad \int_{r_{i n}}^{r_{e n}} \frac{d r}{U(r)}=\int_{t_{i n}}^{t_{e n}} d t=T
$$

which is known from the image and timing data. We can also measure the corresponding propagation distance $r_{e n}$, which leaves us to solve

$$
\int_{r_{i n}}^{r_{e n}} \frac{d r}{U(r)}-T=0
$$

for a given pressure-radius relation. Applying the acoustic and empiric relationships from above

$$
P_{a c}(r)=P_{i n} \frac{r_{i n}}{r}, \quad P_{e m}(r)=P_{i n}\left(\frac{r_{i n}}{r}\right)^{1.13},
$$


Kap. 6 Shock wave and cavitation generation after impact of high-speed projectiles

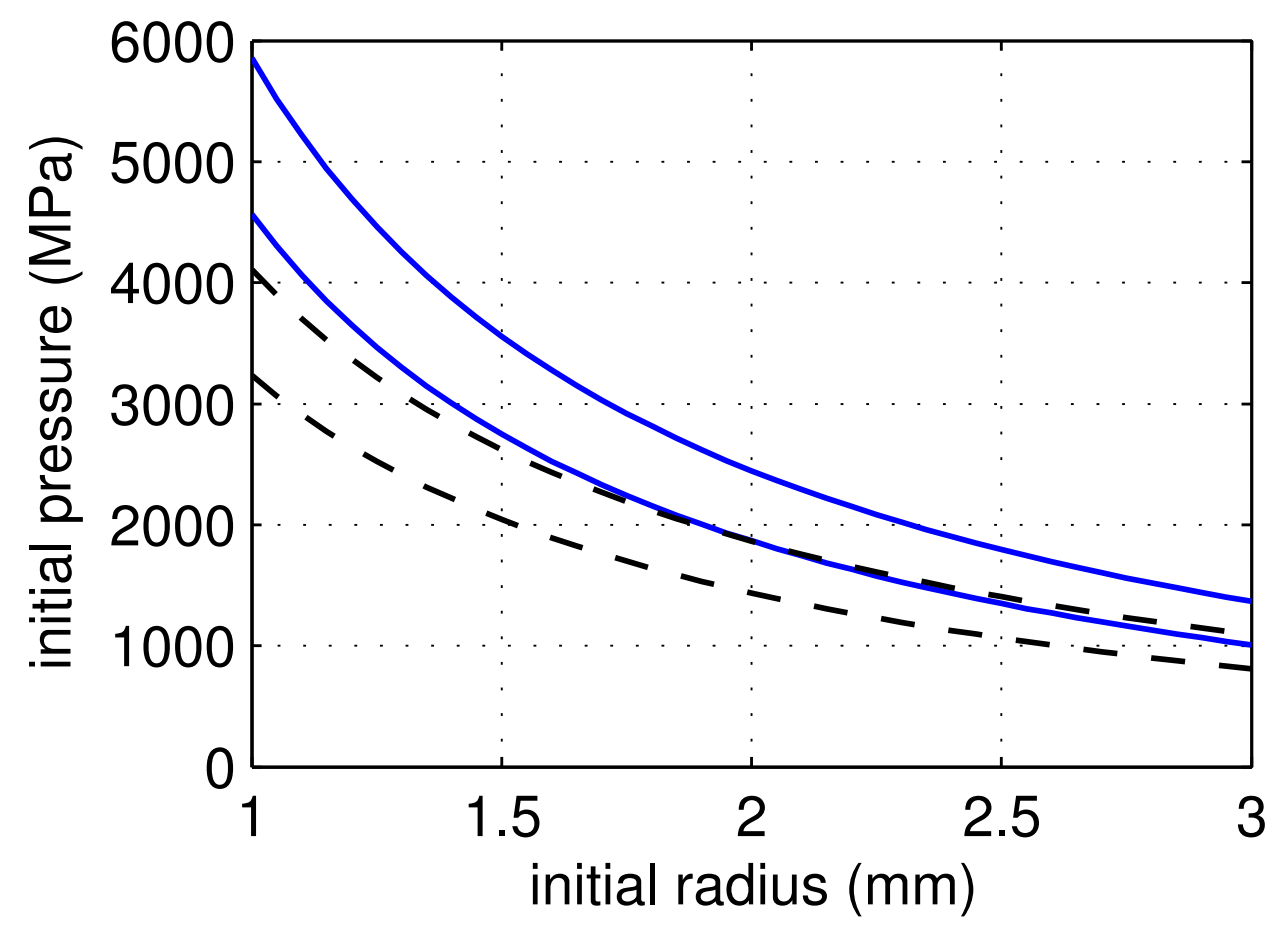

Figure 6.12: Initial pressure depending on the initial radius for a spherical shock wave after bullet impact. The results have been obtained from the location of the shock wave at a specific time $T$ as obtained from image data (Fig. 6.11). The blue curves display the empirical relation $P(r) \sim 1 / r^{1.13}$ derived from pressure measurements after the detonation of TNT charges under water [64], while the dashed black curves represent the acoustic approximation $P(r) \sim 1 / r$. Upper and lower curves account for uncertainties in measurements of the location of the shock wave. 
equation (6.4) has been solved for $P_{\text {in }}$ with initial radii $1 \leq r_{i n} \leq 3 \mathrm{~mm}$ using a simple optimization routine. The results are displayed in Fig. 6.12. At an initial distance of $r_{i n}=2.78 \mathrm{~mm}$ the pressure amounts to $P_{i n, a c}=802 \pm 137 \mathrm{MPa}$ and $P_{e m, a c}=1003 \pm 175 \mathrm{MPa}$ for the acoustic and empirical pressure-radius relationship, respectively. Theses results are slightly higher but in reasonable agreement with the values obtained from the pressure measurement.

Upon reaching the upper boundary of the vessel, the shock wave is reflected and inverted at the water-air interface. The reflected shock wave from the upper boundary can be seen in frames 3 to 6 of Fig. 6.7. In the wake of the tensile wave cavitation bubbles are formed due to the strong negative pressure. Figure 6.8 shows the impact of a high-speed bullet on a smaller flask with different interframe times. In frames 3 to 5 we see again the shock wave with the reflected tensile wave followed by a cloud of cavitation bubbles. The primary cavity continues to grow and eventually surpasses the height of the vessel. In fact, the splash after explosion of the vessel reaches several meters in forward and backward direction.

The negative amplitude of the tension generated after reflection of the shock wave can be focused in a cylindrical target. When shooting in the center of the bottom of a water filled bottle, the negative amplitude of the tensile wave is increased by converging on the central axis. Therefore, the high negative amplitude leads to a dense cavitation cloud on the central axis of the bottle, as shown in frame 8 of Fig. 6.13. Due to the cylindrically symmetric geometry this scenario can be modeled in a 2-dimensional simulation. Expressing equations (3.1) and (3.3) in a cylindrically symmetric coordinate system we get:

$$
\begin{gathered}
\frac{\partial \rho}{\partial t}+\frac{\rho u_{r}}{r}+u_{r} \frac{\partial \rho}{\partial r}+\rho \frac{\partial u_{r}}{\partial r}+u_{z} \frac{\partial \rho}{\partial z}+\rho \frac{\partial u_{z}}{\partial z}=0 \\
\frac{\partial u_{r}}{\partial t}+u_{r} \frac{\partial u_{r}}{\partial r}+u_{z} \frac{\partial u_{r}}{\partial z}+\frac{n \rho^{n-2}}{\rho_{0}^{n}}\left(P_{0}+B\right) \frac{\partial \rho}{\partial r}=0 \\
\frac{\partial u_{z}}{\partial t}+u_{r} \frac{\partial u_{z}}{\partial r}+u_{z} \frac{\partial u_{z}}{\partial z}+\frac{n \rho^{n-2}}{\rho_{0}^{n}}\left(P_{0}+B\right) \frac{\partial \rho}{\partial z}=0
\end{gathered}
$$

with $u_{r}$ and $u_{z}$ being the radial and axial fluid particle velocity, respectively. Equations (6.5) together with the pressure-density relation given by the equation of Tait (3.3) have been calculated for a scenario similar to Fig. 6.13. Thereby the pressure pulse measured with the fiber-optic hydrophone is used as time dependent boundary condition for a finite element calculation. Figure 6.14 shows the results for the shock wave propagation up to $80 \mu$ s after bullet impact. The pressure is color coded with red and blue indicating 
Kap. 6 Shock wave and cavitation generation after impact of high-speed projectiles

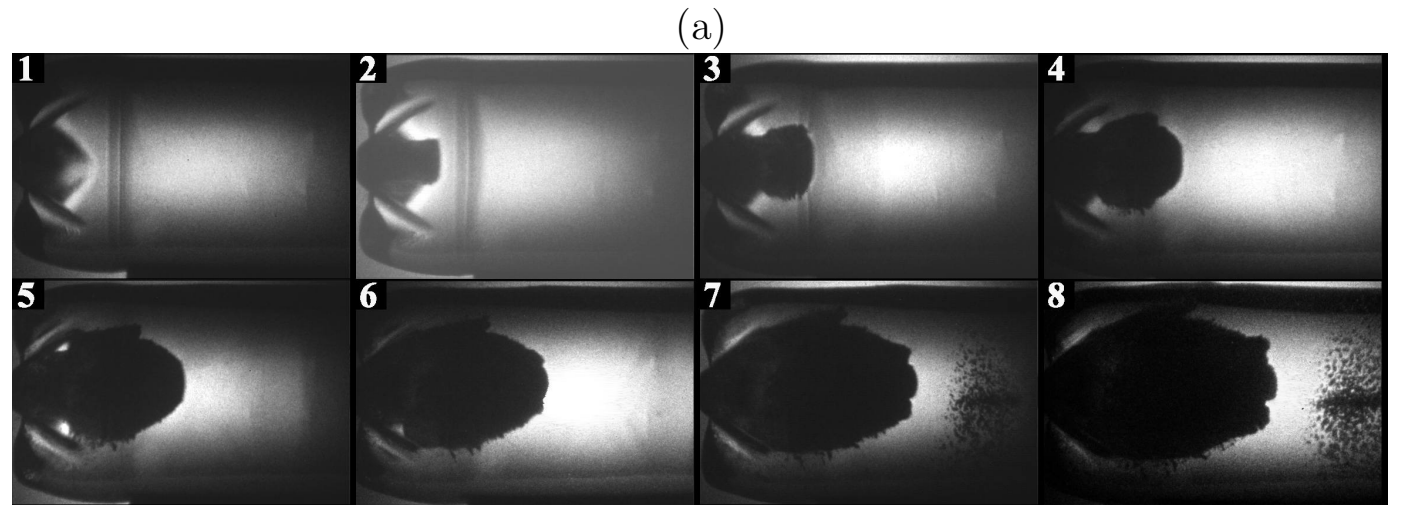

(b)

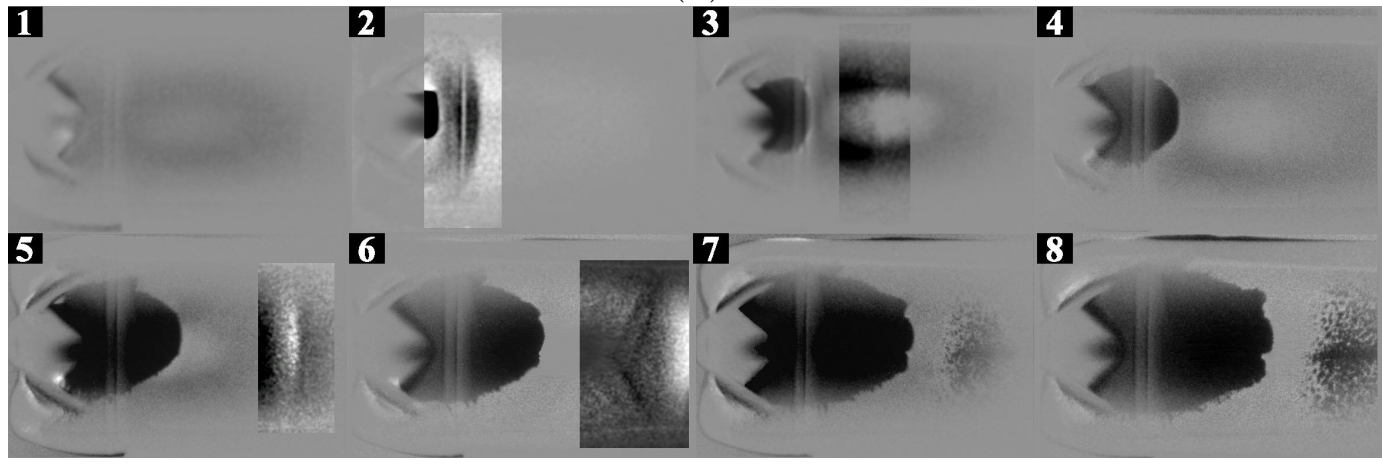

Figure 6.13: Impact of a high-speed projectile $(\approx 1120 \mathrm{~m} / \mathrm{s})$ in the bottom of a water filled bottle. Part (b) shows the background corrected frames of (a) with partly enhanced contrast to better visualize the shock wave. Exposure and interframe times are $2 \mu \mathrm{s}$ and $14 \mu \mathrm{s}$, respectively. The size of a single frame is $137 \times 92 \mathrm{~mm}^{2}$. 
areas of high and low pressures, respectively. The upper boundary is the axis of symmetry. All other straight boundaries are in contact with air, so that the boundary condition $P=P_{0}$ has been used in the model. At the curved line the time dependent pressure fitted from the measurement has been applied as a boundary condition. Thereby it was assumed that the pressure in the medium is uniform on a sphere around the impact site. The radius of the sphere is given by the distance of the hydrophone probe to the location of bullet impact. The simulations should be seen more as a qualitative explanation of the image data, since inaccuracies arise when using a finite element discretization to an almost discontinuous phenomenon like shock wave propagation. Furthermore, the expansion of the primary cavity and the generation of cavitation are not included in the model. The gray overlayed areas mark the maximum length and width of the primary cavity, where the model solutions do not apply. However, results of the simulation mirror the shock wave propagation seen before in the high-speed images. Figure 6.14 shows that the shock wave propagates radially outward until the boundary of the vessel is reached. Here we have a low acoustic impedance reflection and the pressure is inverted. The negative pressure is then focused on the central axis following behind the primary positive pressure pulse. This conforms to the dense cavitation generation on the central axis seen in Fig. 6.13. Also the observed propagation speed of the pressure pulse is in good agreement with the numerical results.

A second simulation giving similar results to the previous one is shown in Fig. 6.15. In here the boundary condition on the curved line has been obtained by assuming a linear increase in pressure in the direction of bullet propagation. References are given by setting the pressure at the left end of the boundary to zero and using the hydrophone measurement to determine the pressure on the boundary $10 \mathrm{~mm}$ away from the axis of symmetry. The initial maximum pressure values obtained in this simulation are slightly larger than using uniform condition along the curved boundary because a higher pressure is applied on the boundary close to the symmetry axis. On the other hand, the pressure generated at the lower part of the curved boundary is much smaller than in the uniform case. It therefore contributes less to the negative amplitude of the reflected pressure wave. However, the characteristics of the initial pressure wave and its reflection on the pressure release boundary are the same in both simulations.

It was already shown, by the comparison of Figs. 6.2 (a) and (b) that "normal-speed" bullets exhibit much less violent behavior than high-speed bullets when shot at melons. Shooting a bullet with almost $900 \mathrm{~m} / \mathrm{s}$ at water it was not possible to directly observe shock waves as seen for high-speed projectiles of Figs. 6.7, 6.8, and 6.13. However, the 
Kap. 6 Shock wave and cavitation generation after impact of high-speed projectiles

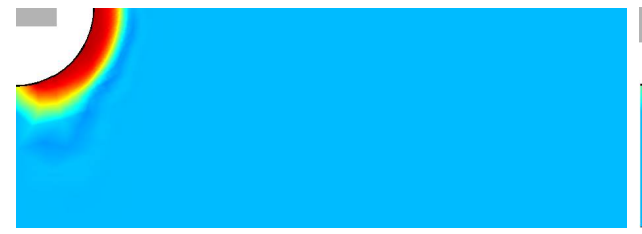

$10 \mu \mathrm{s}$

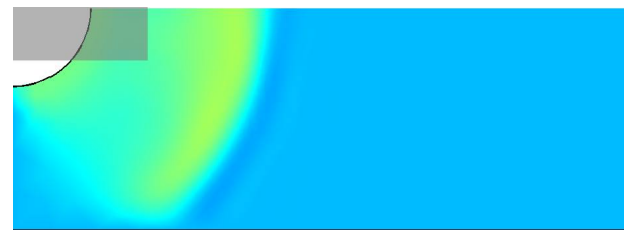

$30 \mu \mathrm{s}$

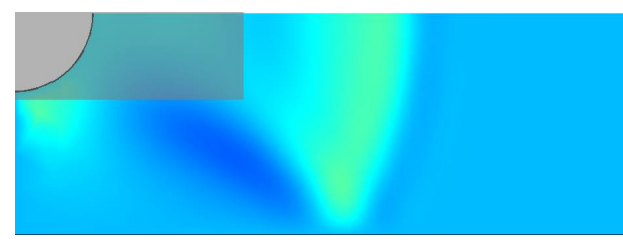

$50 \mu \mathrm{s}$

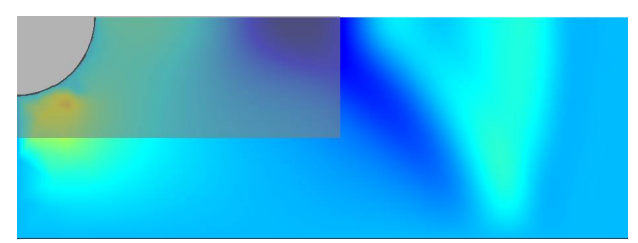

$70 \mu \mathrm{s}$

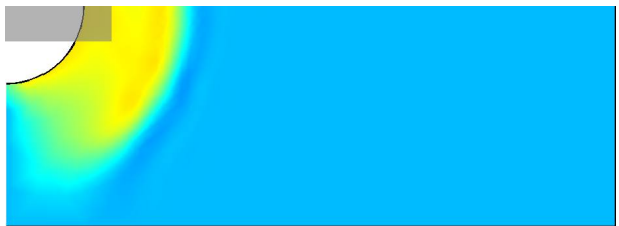

$20 \mu \mathrm{s}$

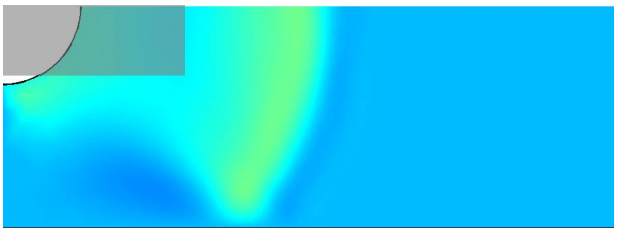

$40 \mu \mathrm{s}$

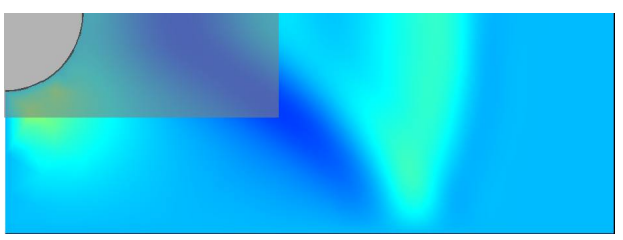

$60 \mu \mathrm{s}$

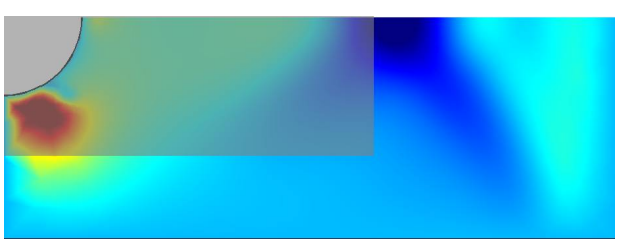

$-20$

60

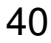

20

0

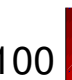

80

$-40$

Figure 6.14: Numerical results of pressure propagation at indicated times in a cylindrically symmetric geometry after bullet impact. The upper edge is the axis of symmetry. In the experiment, the pressure has been measured with an optical hydrophone. The location of the pressure probe concurs with a point on the curved boundary of the model. The results of the pressure measurement were fitted and used as a time dependent boundary condition assumed to be uniform on the curved boundary. All other boundaries are in contact with air and therefore set to atmospheric pressure. The color code represents the pressure with values given in MPa. Gray areas indicate the approximate maximum vertical and horizontal elongation of the primary cavity as obtained from recorded high-speed image sequences. The size of a single frame is $130 \times 47 \mathrm{~mm}^{2}$. 


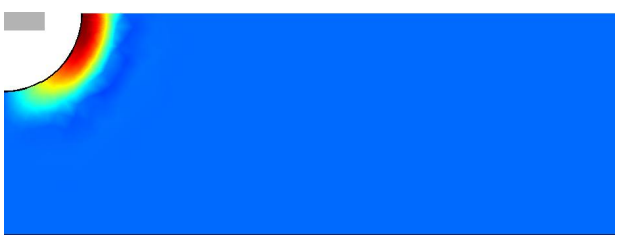

$10 \mu \mathrm{s}$

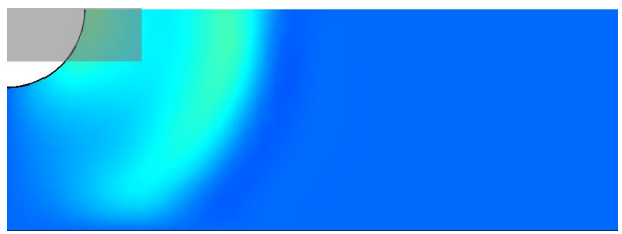

$30 \mu \mathrm{s}$

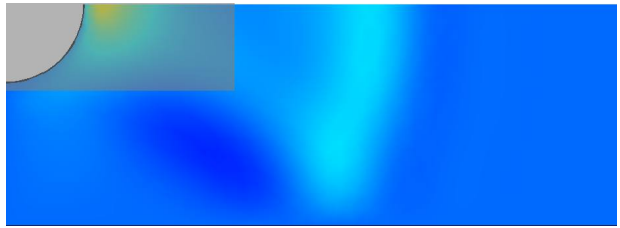

$50 \mu \mathrm{s}$

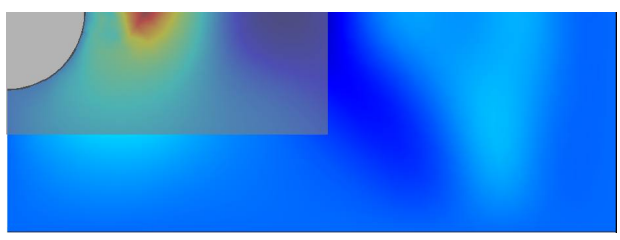

$70 \mu \mathrm{s}$

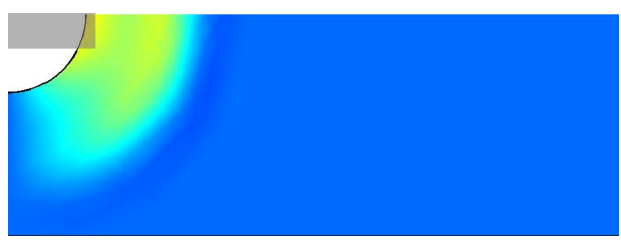

$20 \mu \mathrm{s}$

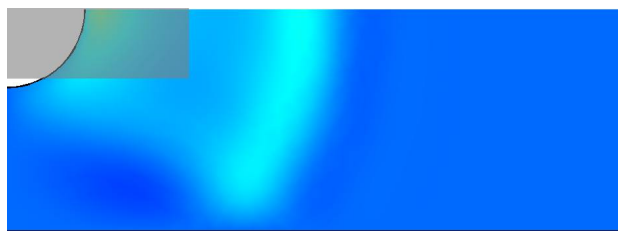

$40 \mu \mathrm{s}$

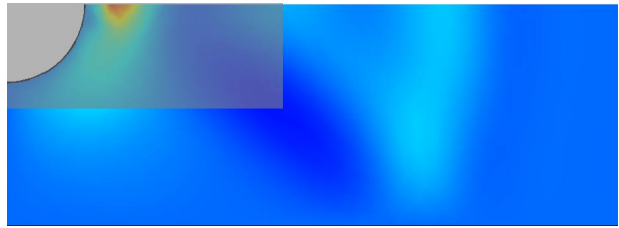

$60 \mu \mathrm{s}$

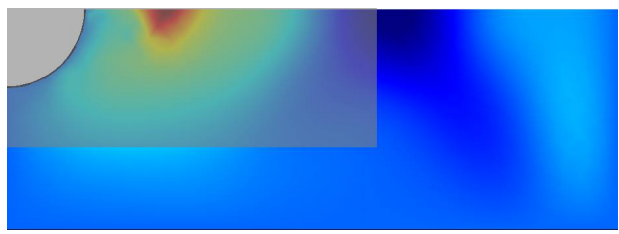

$80 \mu \mathrm{s}$

Figure 6.15: Numerical results of pressure propagation at indicated times in a cylindrically symmetric geometry after bullet impact incident from the left. The upper line is the axis of symmetry. In the experiment, the pressure has been measured with an optical hydrophone. The location of the pressure probe concurs with a point on the curved boundary of the model. The results were fitted and used as a time dependent boundary condition on the curved line. The pressure on the boundary has been assumed to increase linearly in the propagation direction of the bullet being zero at the left end of the boundary and reaching its maximum at the axis of symmetry in the bullet path. All other boundaries are in contact with air and therefore set to atmospheric pressure. The color code represents the pressure with values given in MPa. Gray areas indicate the approximate maximum vertical and horizontal elongation of the primary cavity as obtained from recorded high-speed image sequences. The size of a single frame is $130 \times 47 \mathrm{~mm}^{2}$. 
Kap. 6 Shock wave and cavitation generation after impact of high-speed projectiles

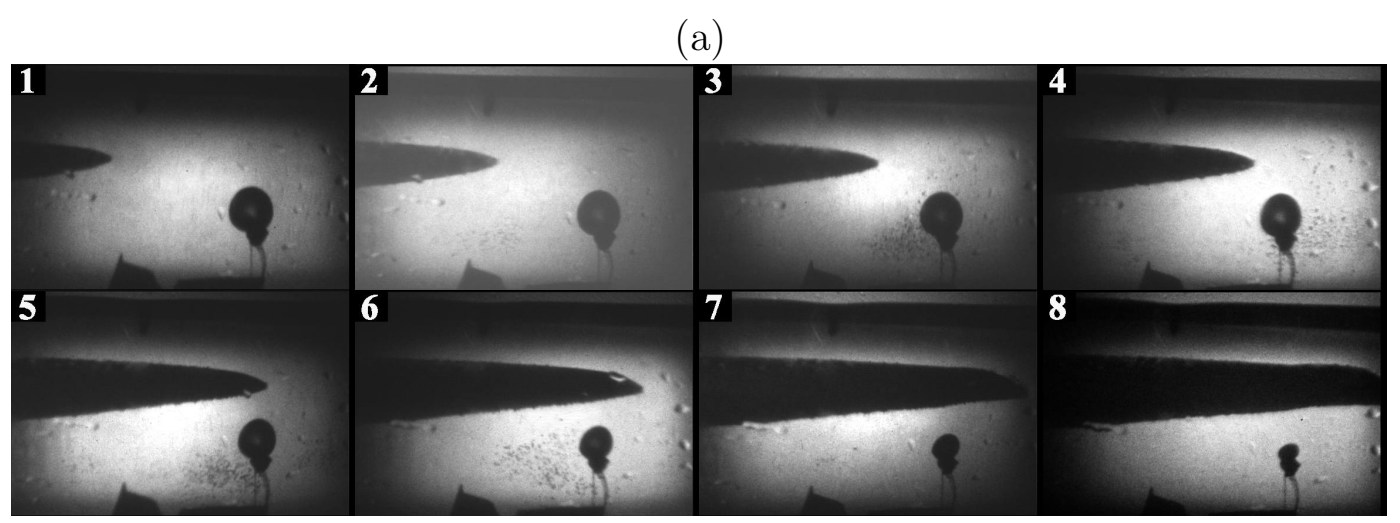

(b)

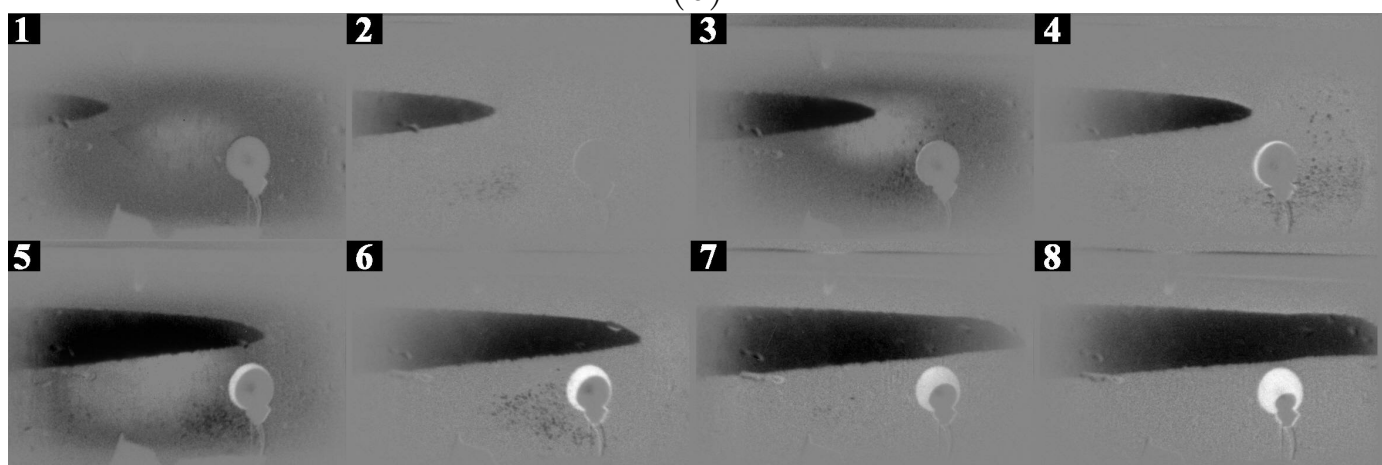

Figure 6.16: Impact of a projectile $(886 \mathrm{~m} / \mathrm{s})$ in the bottom of a water filled bottle. A balloon inside the bottle is compressed by the emitted pressure wave. Part (b) shows the background corrected frames of (a). The initial diameter of the balloon is $\approx 18 \mathrm{~mm}$. Exposure and interframe times are $1.5 \mu \mathrm{s}$ and $16 \mu \mathrm{s}$, respectively. The size of a single frame is $137 \times 92 \mathrm{~mm}^{2}$.

existence of a pressure wave could be detected indirectly, by placing a cavity inside the vessel. In Fig. 6.16 we see a water filled bottle with an air balloon placed inside it. A bullet is shot at $886 \mathrm{~m} / \mathrm{s}$ in the bottom of the bottle. The presence of a pressure wave is revealed by the compression of the balloon, which starts shortly before the passage of the projectile. Cavitation arises from the reflection of the pressure wave at the balloon interface. The initial diameter of the balloon is $\approx 18 \mathrm{~mm}$. Due to compression the diameter decreases to $\approx 7.5 \mathrm{~mm}$ in frame 8 of Fig. 6.16. Assuming spherical geometry this corresponds to a volume decrease of $86 \%$. The compression of the balloon and the cavitation generation after pressure wave reflection indicate the importance of cavities (e.g. the lungs) inside the body when regarding shock wave damage in humans.

Suneson et. al. reported on microscopic scattered central and peripheral nervous dam- 
age following high-velocity missile wounds in the thigh of pigs [139]. Cavitation bubbles generated by a tensile pulse might be responsible for such a scattered damage characteristics. The strength of a tensile pulse after shock wave reflection may vary due to focusing effects dependent on the geometry of low acoustic impedance boundaries.

\subsection{Conclusion}

In this chapter the impact of high-speed projectiles on gelatin and water and secondary effects were investigated. It was shown that full metal jackets deform and fragment when shot at speeds over $1000 \mathrm{~m} / \mathrm{s}$ resulting in a much more violent scenario than seen for slower projectiles. Upon bullet impact a shock wave is created, which expands into the medium. Image data shows the generation of cavitation after shock wave reflection at a water air interface. Pressure measurements were used in a finite element calculation to simulate wave propagation and reflection in an axisymmetric geometry. The numerical results are in good agreement with the shock wave propagation seen in high-speed images and explain the locations of cavitation generation. From the experiments it seems possible that cavitation generation is responsible for scattered damage far from the initial site of bullet impact. The occurrence of strong tensile waves responsible for cavitation generation may be enhanced due to focusing effects after shock wave reflection at pressure release boundaries. 


\section{Chapter 7}

\section{Interactions of adherent cells with shock wave induced cavitation}

In Chapter 5 it was already shown that pressure excited microbubbles may cause damage to cells in their vicinity. Now the focus is placed on the effects of lithotripter induced cavitation on cells adherent to a substrate. Main emphasis is thereby placed upon transient membrane permeabilization and cell detachment. The experiments described in this chapter were conducted in collaboration with Claus-Dieter Ohl at the University of Twente [140].

\subsection{Materials and methods}

The methods used to perform the experiments comprise biochemical and physical techniques. Physical practices include high-speed imaging and shock wave creation and measurements. Biochemical assays were conducted after shock wave exposure to investigate transient and permanent membrane permeabilization.

\subsubsection{Cell culture}

Human cervix epithelia carcinoma cells (HeLa) were grown at $37^{\circ} \mathrm{C}$ and $5 \% \mathrm{CO}_{2}$ in Iscove's Modified Dulbecco's medium (Invitrogen). The medium was supplemented with $10 \%$ fetal bovine serum, antibiotics (100 units/ml penicillin, $100 \mu \mathrm{g} / \mathrm{ml}$ streptomycin), and 0.25 $\mu \mathrm{g} / \mathrm{ml}$ amphotericin (Invitrogen). Cells were split with trypsin-EDTA (0.05\%, Invitrogen) 1 to 3 days before use and plated in polystyrol culture flasks (Nunc EasYFlask, Fisher) 


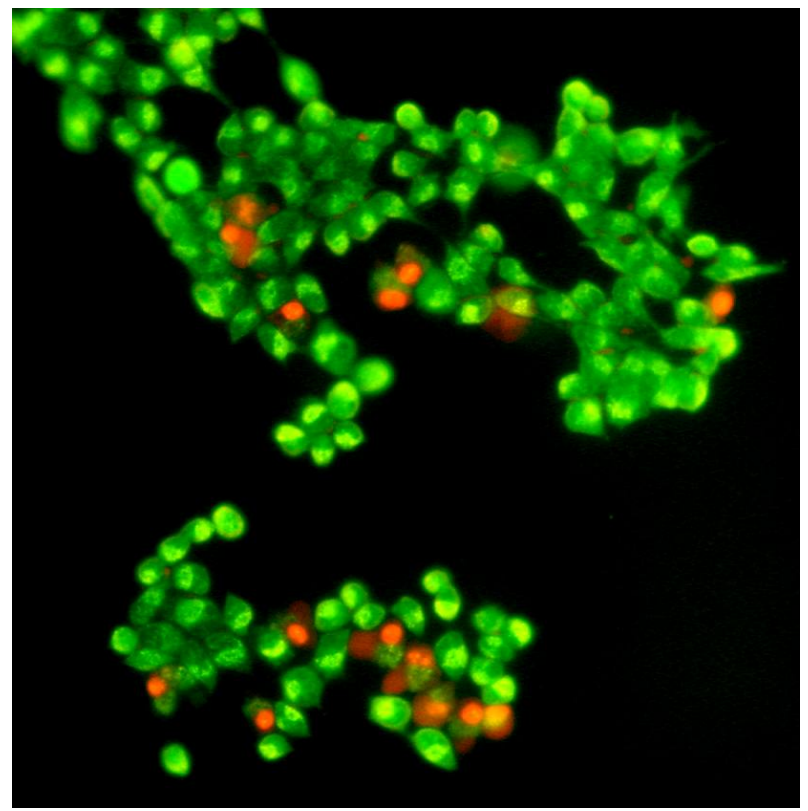

Figure 7.1: Viability assay of cells after shock wave exposure. Ruptured cells appear red or orange under fluorescence excitation due to the intercalation of ethidium bromide into the cell's DNA. Cells with intact membranes are stained green by intercalation of acridine orange into the DNA.

of $25 \mathrm{~cm}^{2}$ growth area and $60 \mathrm{ml}$ volume. The cell doubling time was approximately 48 hours. The flasks were filled up with medium prior to shock wave exposure.

\subsubsection{Viability stain}

After shock wave exposure the suspension containing detached cells was removed carefully and subsequently centrifuged for 5 minutes at $200 \mathrm{~g}$. The supernatant medium was removed and cells were resuspended in $1 \mathrm{ml}$ phosphate buffered saline (PBS, Sigma). Afterwards cells were tested for viability using ethidium bromide/acridine orange (Fluka) staining at final concentrations of $5 \mu \mathrm{g} / \mathrm{ml}$ and $1.5 \mu \mathrm{g} / \mathrm{ml}$, respectively. Ruptured cells appear orange due to the intercalation of ethidium bromide into their DNA and RNA. Cells with intact membranes are stained green by the membrane-permeant dye acridine orange. An example image of ruptured and intact cells after this staining procedure is given in Fig 7.1. Dead and alive cells were counted in a Neubauer chamber under a fluorescence microscope (Zeiss). 


\subsubsection{Transient membrane permeabilization}

Transient membrane permeabilization was assayed by the uptake of fluorescein isothiocyanate dextran (FITC-dextran, $20 \mathrm{kDa}$, Sigma) or calcein (623 Da, Sigma). Non membrane-permeant FITC-dextran $(1 \mathrm{mg} / \mathrm{ml})$ or calcein $(1 \mathrm{mg} / \mathrm{ml})$ was added to the cell medium before shock wave exposure. Dye uptake of attached cells was determined by fluorescence microscopy after washing the cells four times with PBS and adding ethidium bromide at a final concentration of $5 \mu \mathrm{g} / \mathrm{ml}$. Transiently porated cells, loaded with FITCdextran or calcein appear green under fluorescence excitation. Permanently damaged cells appear orange due to ethidium bromide staining. Detached cells were treated with the same procedure as described for viability staining. However no acridine orange was used because it would not be possible to distinguish between living cells with or without FITC-dextran uptake.

\subsubsection{Shock wave generation and microscopic observation}

Shock waves were generated by a focused piezoceramic source, which was adapted from the commercial lithotripter Piezolith 3000 (Richard Wolf GmbH). The lithotripter contains two piezoceramic layers with a diameter of $300 \mathrm{~mm}$ and a focusing angle of $94^{\circ}$. The lithotripter was operated at voltages between $3.5 \mathrm{kV}$ and $7 \mathrm{kV}$ and only the frontal piezoceramic layer was used in the experiments. The pressure was measured at the lithotripter focus in the free field and behind the substrate of the culture flask with a calibrated needle type hydrophone of $1 \mathrm{~mm}$ diameter (Dr. Pecha, University of Stuttgart). The sensitive area of the hydrophone consists of a polyvinylidene fluoride (PVDF) foil of $20 \mu \mathrm{m}$ thickness. A typical recording in the free field at an operating voltage of $6 \mathrm{kV}$ is presented in Fig. 7.2. Due to the limited bandwidth of the hydrophone, the fast rise time cannot be resolved. Also the formation of cavitation bubbles at the hydrophone tip causes an underestimation of the duration of the negative pressure tail. However, the main features of the pressure pulse are resolved: The wave consists of a positive pressure pulse followed by a negative tail. The positive pressure rises steeply and lasts for approximately $1.5 \mu \mathrm{s}$. Maximum amplitudes range between $10 \mathrm{MPa}$ and $40 \mathrm{MPa}$ depending on the discharge voltage. The tensile pressure pulse shows maximum negative amplitudes of around $-4 \mathrm{MPa}$, and lasts for several microseconds. The reproducibility of the waveform was high. Using the same setup positive pressure amplitudes varied less than $5 \%$ from shot-to-shot. Between consecutive shots at least $60 \mathrm{~s}$ were waited so that the bubbles generated had time to 


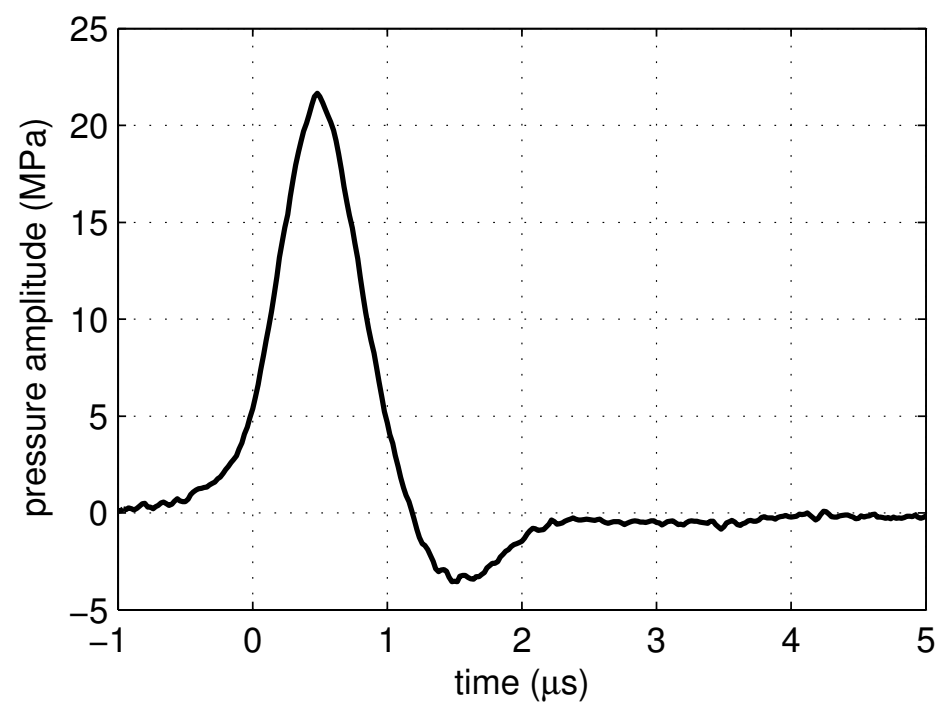

Figure 7.2: Typical free field pressure recording of the lithotripter shock wave. A discharge voltage of $6 \mathrm{kV}$ is applied on the piezoelectric layer of the shock wave generator.

dissolve or float out of the focusing area. A sketch of the experimental setup is presented in Fig. 7.3. The shock wave generator was oriented at an angle of $45^{\circ}$ to the horizontal. The transducer was mounted into the bottom of a basin made of stainless steel with three glass windows. Approximately $50 \mathrm{l}$ of degassed water containing $3 \mathrm{mg} \mathrm{O}_{2} / \mathrm{l}$ were filled into the basin. The spatial position of the focus was first determined with the hydrophone and then marked by adjusting two beams from laser diodes in a way that they cross each other at the tip of the hydrophone. For optical observations a long distance microscope (K2 with a CF4 objective, Infinity), which was attached to a dual frame CCD camera (Imager 3S: $1280 \times 1024$ pixels, LaVision) was used. The camera is typically used for measuring particle displacements in particle image velocimetry (PIV) applications as it can record two separate stroboscopically illuminated frames in rapid succession. In the investigations presented here, this camera type was chosen because of its small pixel size $\left(6.7 \times 6.7 \mu \mathrm{m}^{2}\right)$ combined with a high sensitivity and the ability to record fast events at moderate costs. Alternatively, when it was desired to take multiple images of a larger area, a high-speed video camera with $640 \times 480$ pixels and a framerate of 100 frames/s was used (Pulnix, TM6710). The long distance microscope was encased in a cylindrical glass tube with the front side covered with a $5 \mathrm{~mm}$ thick glass plate. Illumination was accomplished by driving a pulsed light emitting diode (LED) with a home build current amplifier. The system was triggered with a double pulse generator (HP 33120A, Agilent). The setup 


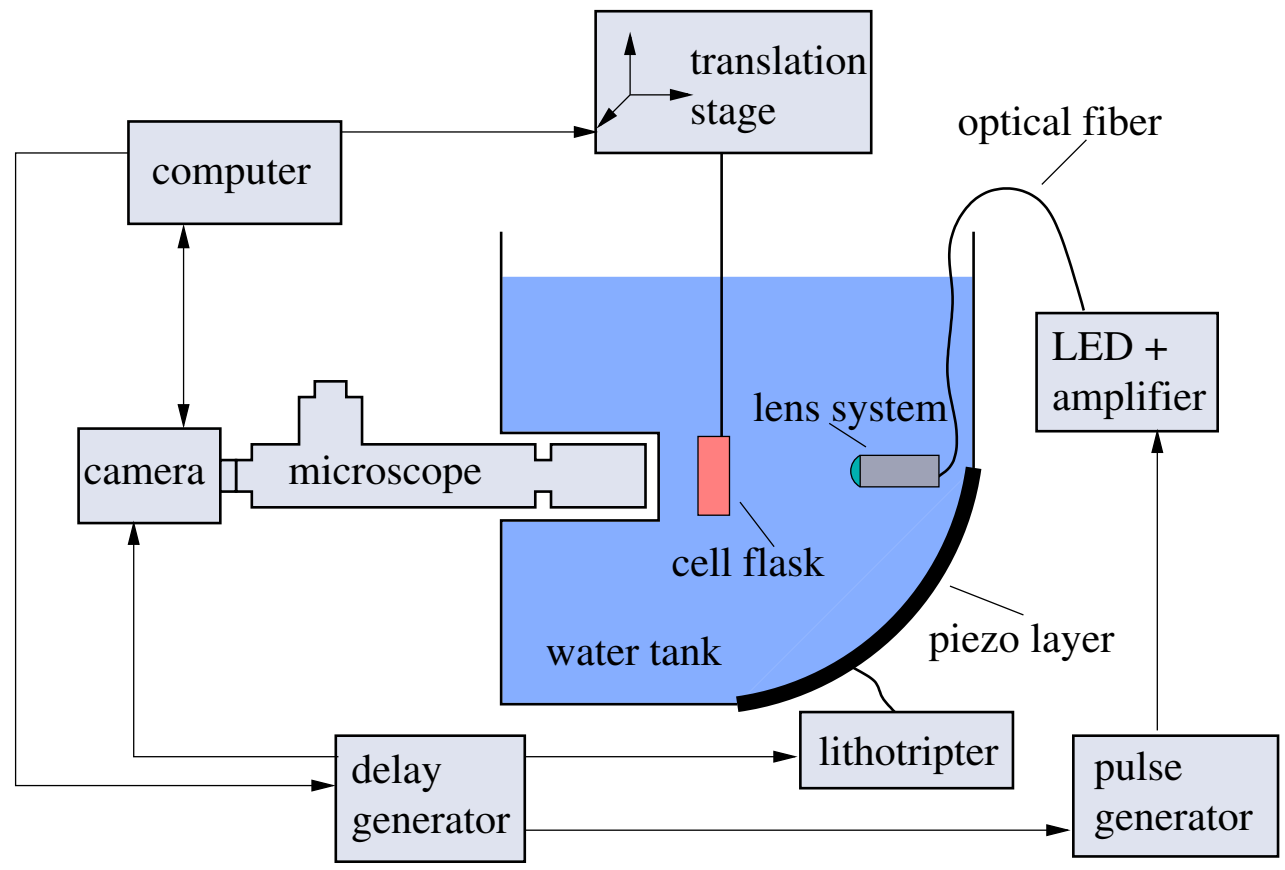

Figure 7.3: General setup for the observation of shock wave induced cavitation in a cell culture flask.

ensured that the amplifier delivers between $1 \mathrm{~A}$ and $3 \mathrm{~A}$ only for a short duration and at very low duty cycles not to damage the semiconductor. Light from the diode was coupled into a $1 \mathrm{~mm}$ core polymethylmetaacrylat (PMMA) fiber and brought to a two-lens-system sealed and submerged into the water bath. The lenses collect and refocus the light while matching the numerical aperture to that of the long-distance microscope. The culture flask containing the cells was attached to a motorized three-axis translation stage (PI) with sub-micrometer resolution. Its position was controlled with a three axis joystick. In most data presented here, the flask was oriented with the cell substrate being first to be hit by the pressure pulse. However, we also conducted experiments with the bottom of the flask facing the camera, which yielded similar results. The transmission coefficient of the substrate has been determined by comparing the peak positive amplitude of the shock wave, $P_{+}$, in presence and absence of the substrate. A damping of approximately $10 \%$ of the amplitude $P_{+}$was found with the substrate oriented perpendicular to the direction of the shock wave propagation. In the cell experiments the angle between substrate and shock wave propagation was $45^{\circ}$ resulting in an amplitude reduction of about $30 \%$ to $50 \%$. The damped and undamped peak amplitudes for this setup are shown in Fig. 7.4. The temperature of the lithotripter bath and the cell medium was kept at approximately $25^{\circ} \mathrm{C}$. 


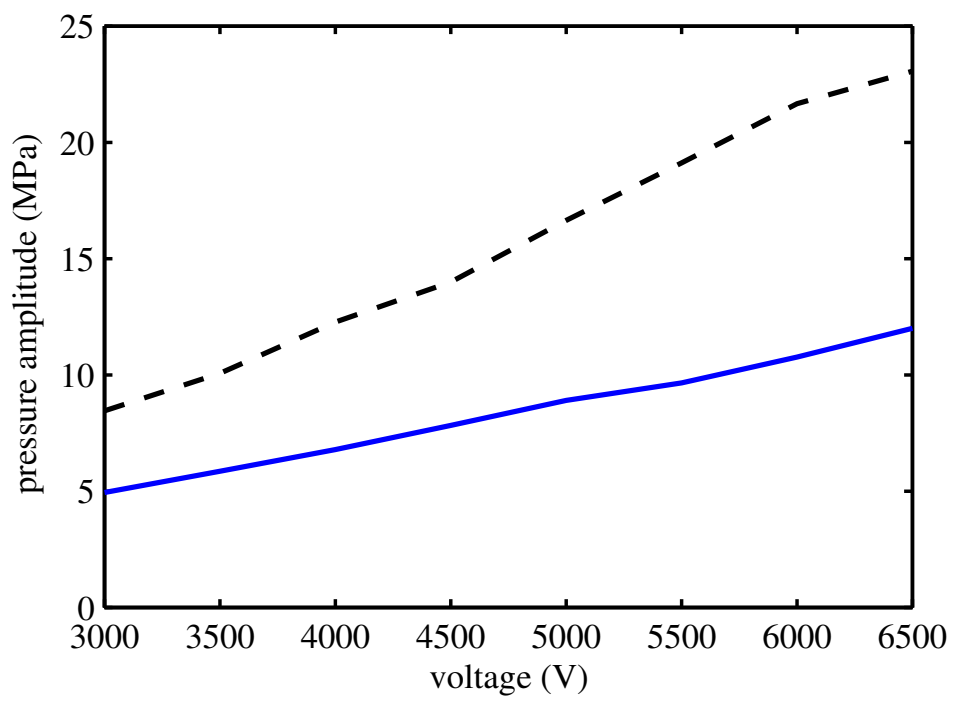

Figure 7.4: Maximum pressure amplitude $P_{+}$of the shock wave as a function of discharge voltage applied to the piezo layer. The pressure was measured with (blue curve) and without (dashed black curve) damping by the culture flask substrate.

\subsection{Cavitation inception}

First, the interaction of the shock wave on the bare substrate of the culture flask is investigated. Generally, in the trailing part of the shock wave cavitation nuclei can grow due to the large tension of several megapascals of negative pressure. A growth of bubbles from these nuclei on the substrate in absence of cells is presented in Fig 7.5. The individual frames correspond to different times of a single experimental run. In the first frame of Fig. 7.5, the inner side of the polystyrol substrate is seen together with impurities related to the action of prior shock wave passages. It is possible that the impurities are remnants of the locally disrupted substrate surface coating supporting the adhesion of cells. The substrate itself does not show cracks even after being subjected to 100 shock waves with the lithotripter working at $7 \mathrm{kV}$. The second frame in Fig. 7.5 is taken $7 \mu \mathrm{s}$ after the shock wave passage. The captured bubbles are blurred due to their explosive growth and the relatively long exposure time of $2 \mu \mathrm{s}$. In the third frame taken ten microseconds later, the bubbles are already expanded to a size of approximately $250 \mu \mathrm{m}$ in diameter. Not shown in the figure is the maximum expansion of the bubbles, which is obtained around 100 to $200 \mu$ s after shock wave passage depending on bubble size. The fourth frame of Fig. 7.5 displays the substrate $516 \mathrm{~ms}$ after shock wave passage. Remnants of the collapsed bubbles either dissolved or floated out of the field of view. Note, that most 

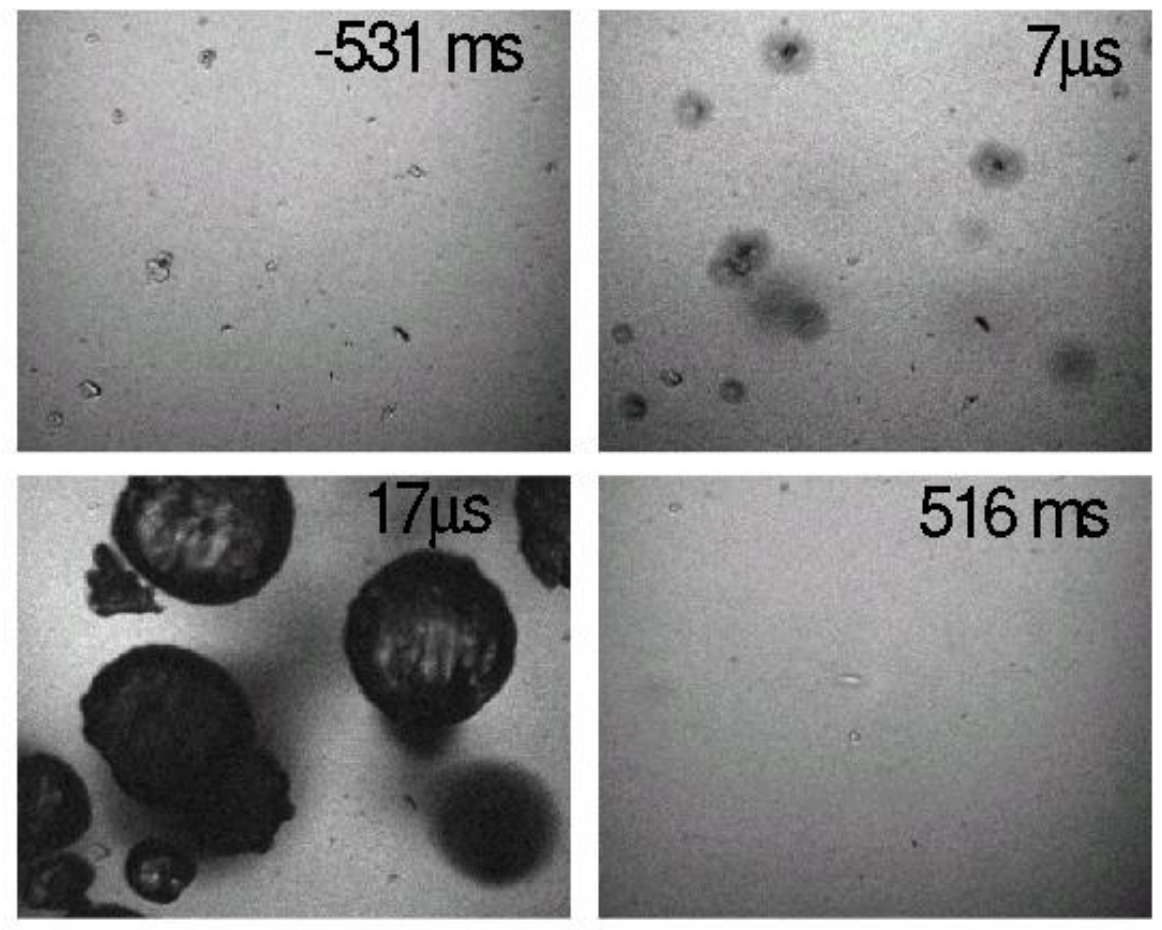

Figure 7.5: Growth of cavitation bubbles on the inside of a culture flask after shock wave passage. The operating voltage of the lithotripter was set to $7 \mathrm{kV}$. Indicated times for each frame are given with reference to the shock wave impact. Exposure times are $2 \mu \mathrm{s}$. The first frame shows the bare substrate with minor defects generated during prior shock wave applications. Cavitation inception occurs $7 \mu$ s after shock wave passage. Large expanding bubbles are visible at $17 \mu$ s. Half a second later the substrate is partly cleared from the defects indicating that the cavitation induced flow field has flushed prior nucleation sites. The frame width is $1 \mathrm{~mm}$. 
of the impurities where cavitation was nucleated have disappeared. They might have been washed away by the strong flow generated during bubble collapse. After application of 2-5 shock waves at constant amplitude with the same focal spot, no further bubbles could be nucleated at that specific site. Only, after increasing the charging voltage of the shock wave generator, or moving the substrate by several millimeters, which is approximately the size of the focal diameter, new bubbles were nucleated again. The general difference in bubble creation compared to Chapter 5 lies in the omission of providing cavitation nuclei. However, the absence of cavitation after several shock wave exposures at the same spot supports the hypothesis of cavitation inception from in some means stabilized microbubbles not visible within the resolution limit of the optical setup [141,107,142].

\subsection{Cell displacement and detachment}

A typical example of the interaction of a cavitation bubble with cells is depicted in Fig. 7.6. Time data is given with respect to the shock wave impact on the substrate. Shortly after the impact we can see a large bubble in the top center of frame 2, which is generated by the tensile pulse following the lithotripter shock wave. Further bubbles appear at the sides of the image. They are blurred either due to their rapid expansion dynamics or because they are not in the focal plane. In frame 3, approximately half a second later, the bubble has collapsed and cells in its vicinity have been detached from the substrate. The last frame is taken $30 \mathrm{~s}$ after shock wave impact. Previously detached cells have flowed out of the region of view leaving the bare substrate at the bubble site.

The question arises at what time during bubble dynamics the cell detachment is caused. In Chapter 5 it was shown that the bubble collapse has the strongest influence on cells nearby. Due to the limited number of high-speed images and the jitter for lithotripter induced bubble collapse time it is more difficult to detect the instant of visible cell reactions in the current setup. However, in Fig. 7.7 it is clearly visible that the main detachment process takes place during or after the bubble collapse. In the upper right corner of frame 2 of Fig. 7.7, $7 \mu$ s after shock wave impact, we can see an expanding bubble. As in frame 2 of Fig. 7.5 the bubble is blurred because of the rapid expansion dynamics and the relatively long exposure time. In the third frame of Fig. 7.7, $237 \mu$ s after shock wave impact, the bubble is blurred again, this time because of the fast contraction upon collapse. Although the bubble is already in the collapse phase we can see that cells are still attached to the substrate. In the next frame approximately half a second later, the 

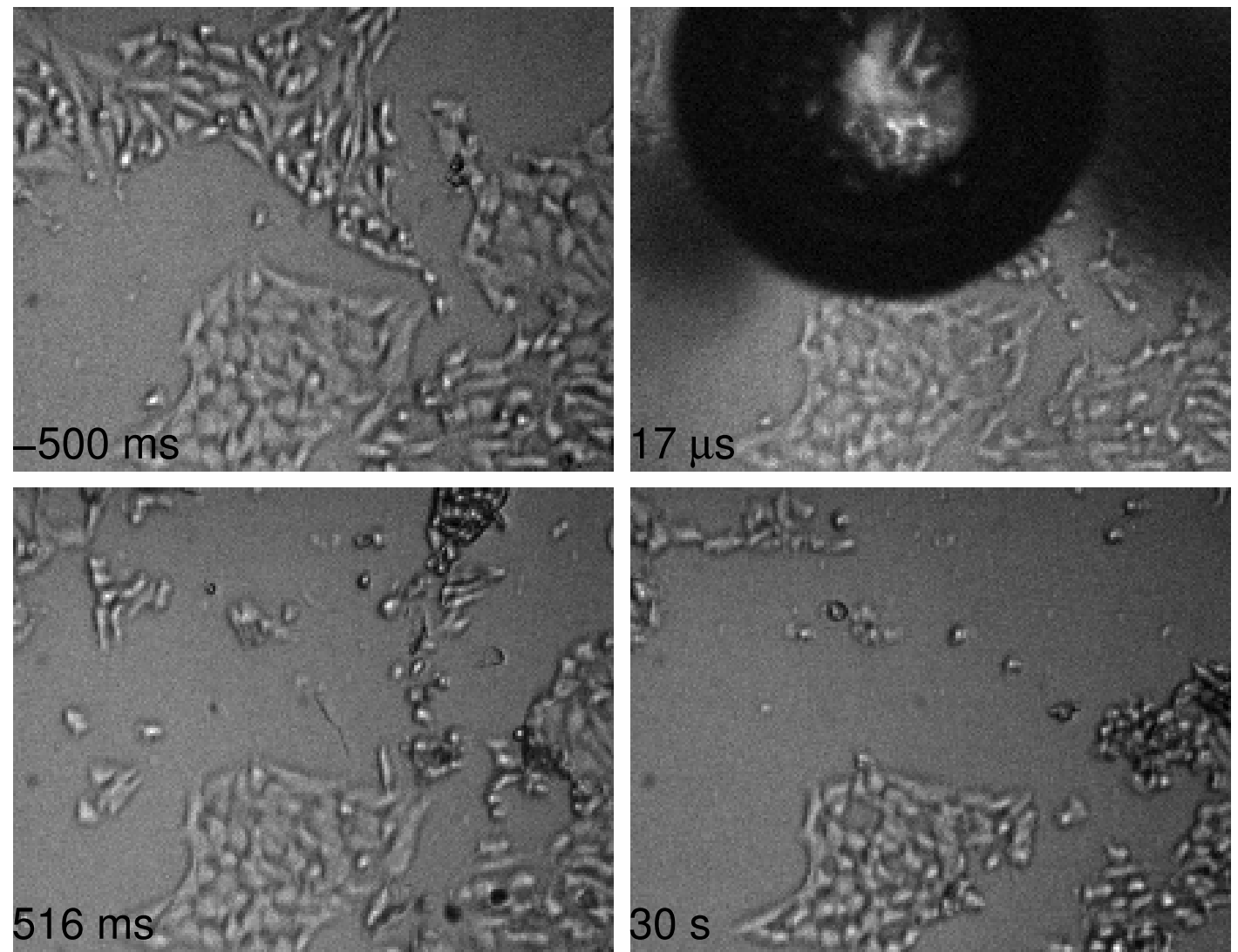

Figure 7.6: Cell detachment caused by a lithotripter induced cavitation bubble. The shock wave generator is operating at $5 \mathrm{kV}$. Time data is given with respect to shock wave impact on the substrate and exposure times are $2 \mu \mathrm{s}$. The frame size is $0.74 \times 0.57 \mathrm{~mm}^{2}$. 

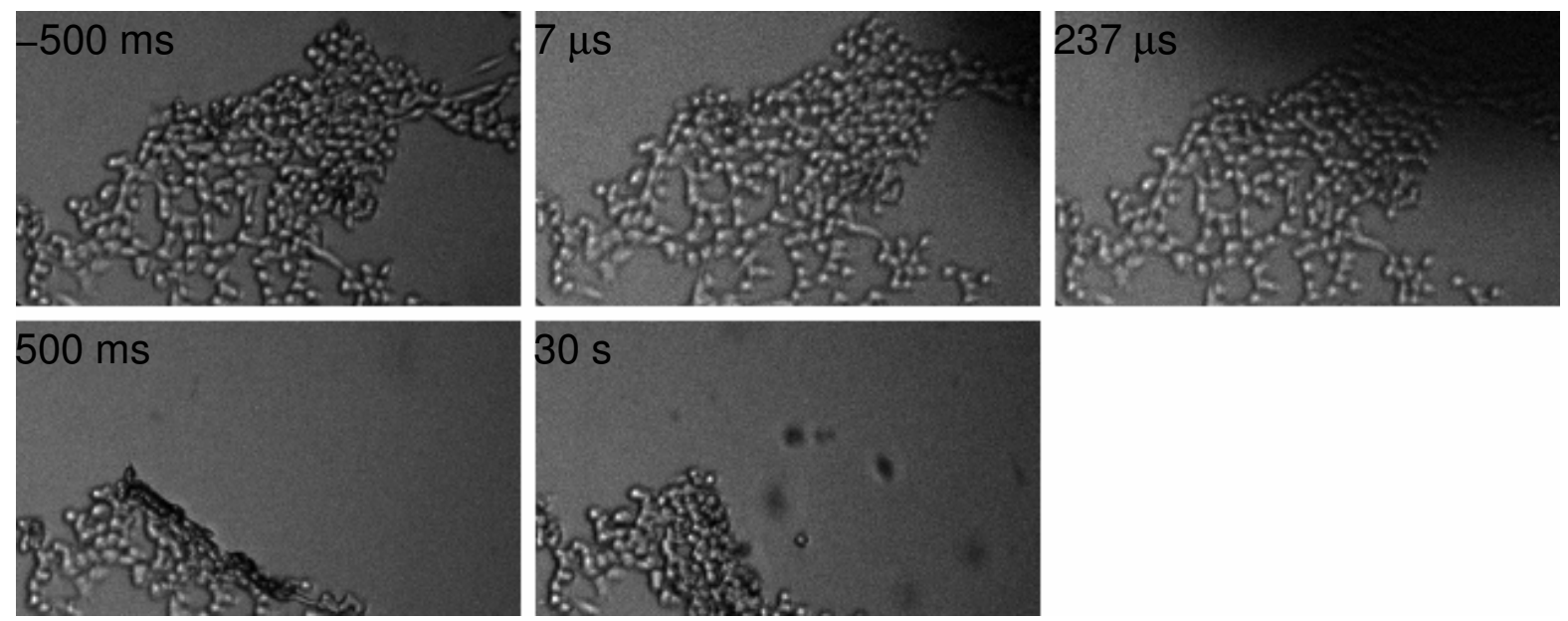

Figure 7.7: Cell detachment caused by the collapse of a lithotripter induced bubble. The bubble is blurred due to its rapid expansion (frame 2) and contraction (frame 3). Time data is given with respect to shock wave impact on the substrate. The lithotripter is operating at $6 \mathrm{kV}$. Exposure times are $2 \mu \mathrm{s}$ and the frame size is $0.69 \times 0.40 \mathrm{~mm}^{2}$.

cells close to the bubble have been pushed away giving rise to a densely packed cell border. After $30 \mathrm{~s}$ the cell agglomeration has somewhat relaxed resulting in a less densely packed alignment.

In the preceding examples the bottom of the flask was facing away from the camera with the substrate being the first side to be hit by the shock wave. Figure 7.8 shows cell detachment by cavitation bubbles with the substrate facing towards the camera. $107 \mu \mathrm{s}$ after shock wave impact we see two bubbles above the cell substrate. The smaller bubble is distorted from spherical shape. It is already in the collapse phase and is influenced by the boundary of the substrate and the neighboring bubble. In frame 3 both bubbles have collapsed and cells in the vicinity of the bubbles are being removed from the substrate. Most cells are detached during the first $500 \mathrm{~ms}$ but some additional cell detachment can be seen in frame 5, $30 \mathrm{~s}$ later. No significant difference in cell detachment depending on the orientation of the flask could be detected. This indicates that the boundary of the substrate or neighboring bubbles have a dominant effect on bubble collapse compared to the propagation direction of the shock wave.

After multiple shock wave application in the same area, the detachment of cells was facilitated. This can be seen in Fig. 7.9 where a densely grown monolayer of cells is shown after 1 to 5 subsequent shock wave exposures at the same spot. Each horizontal 

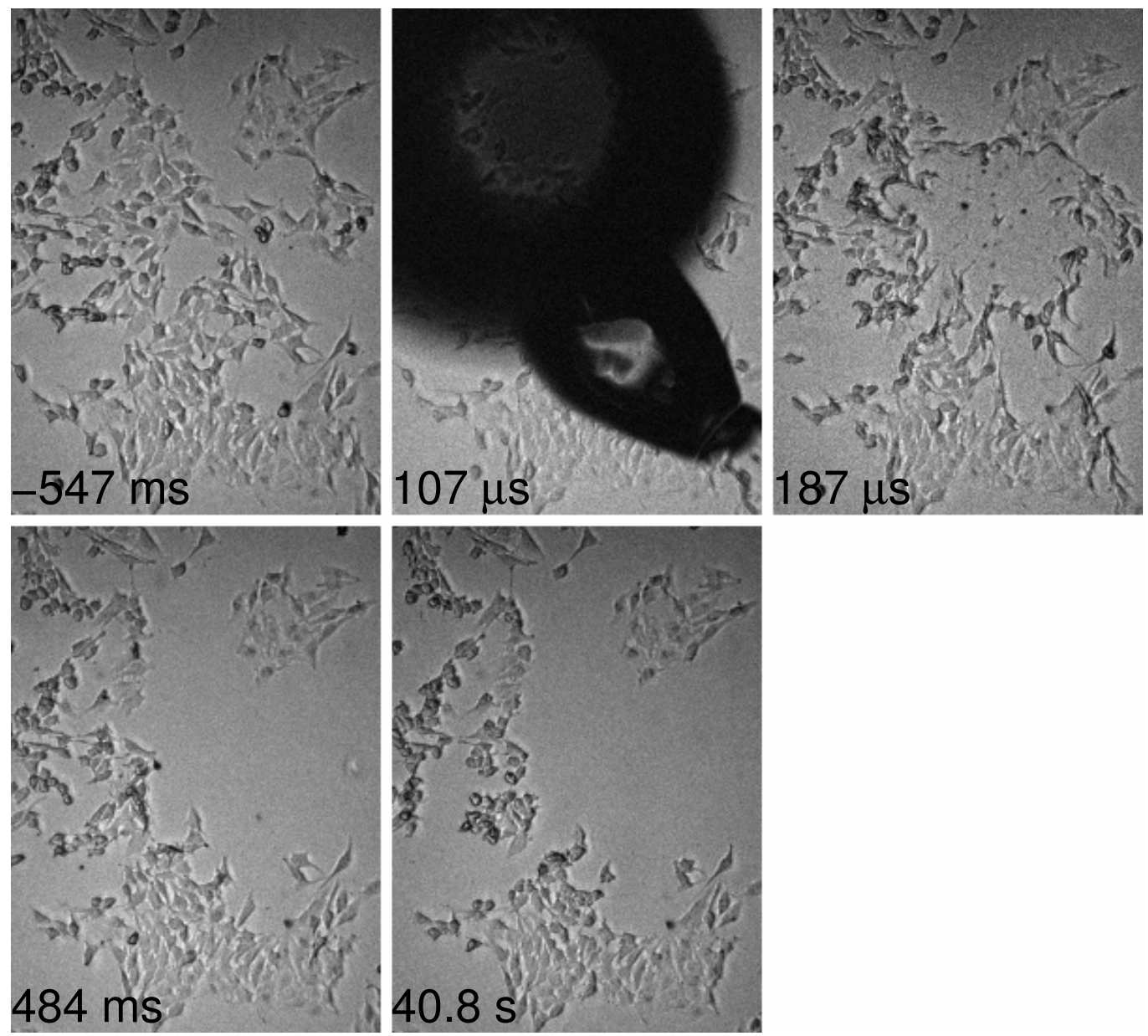

Figure 7.8: Cell detachment caused by lithotripter induced bubble collapse. These images were taken with the bottom of the flask facing towards the camera. The shock wave generator was operated at $6 \mathrm{kV}$. Time data is given with respect to the shock wave impacting on the substrate. The frame size is $0.66 \times 0.90 \mathrm{~mm}^{2}$. Exposure times are $2 \mu \mathrm{s}$. 


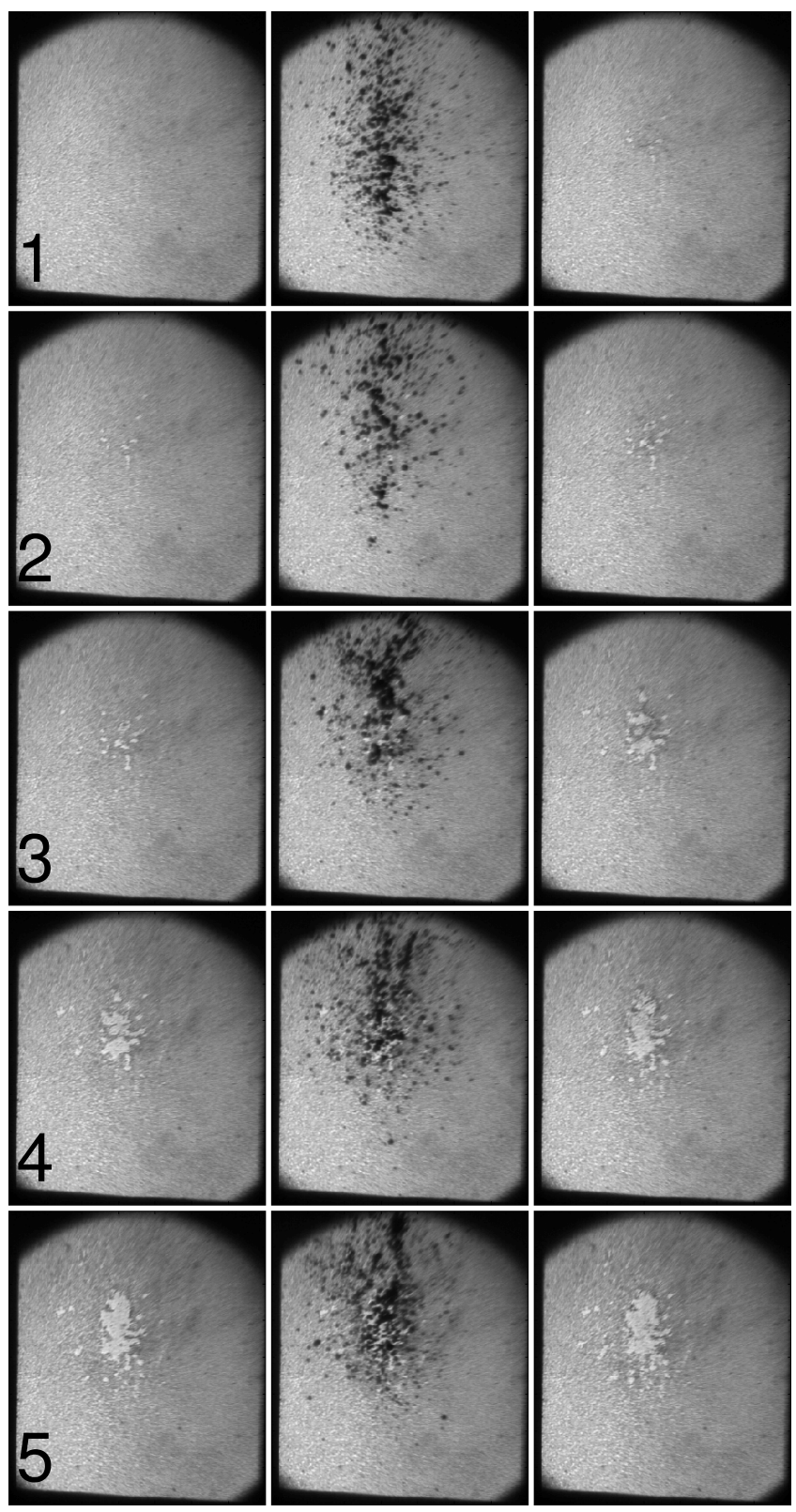

Figure 7.9: Large scale cell detachment after 1 to 5 subsequent shock wave applications. Every horizontal sequence displays the cell monolayer $10 \mathrm{~ms}$ before, during, and $10 \mathrm{~ms}$ after cavitation generation. Brighter regions in the image indicate that cells have been detached from the substrate. The operating voltage of the lithotripter was set to $6 \mathrm{kV}$. Exposure times are $2 \mu \mathrm{s}$ and the frame size covers $3.4 \times 3.9 \mathrm{~cm}^{2}$. 

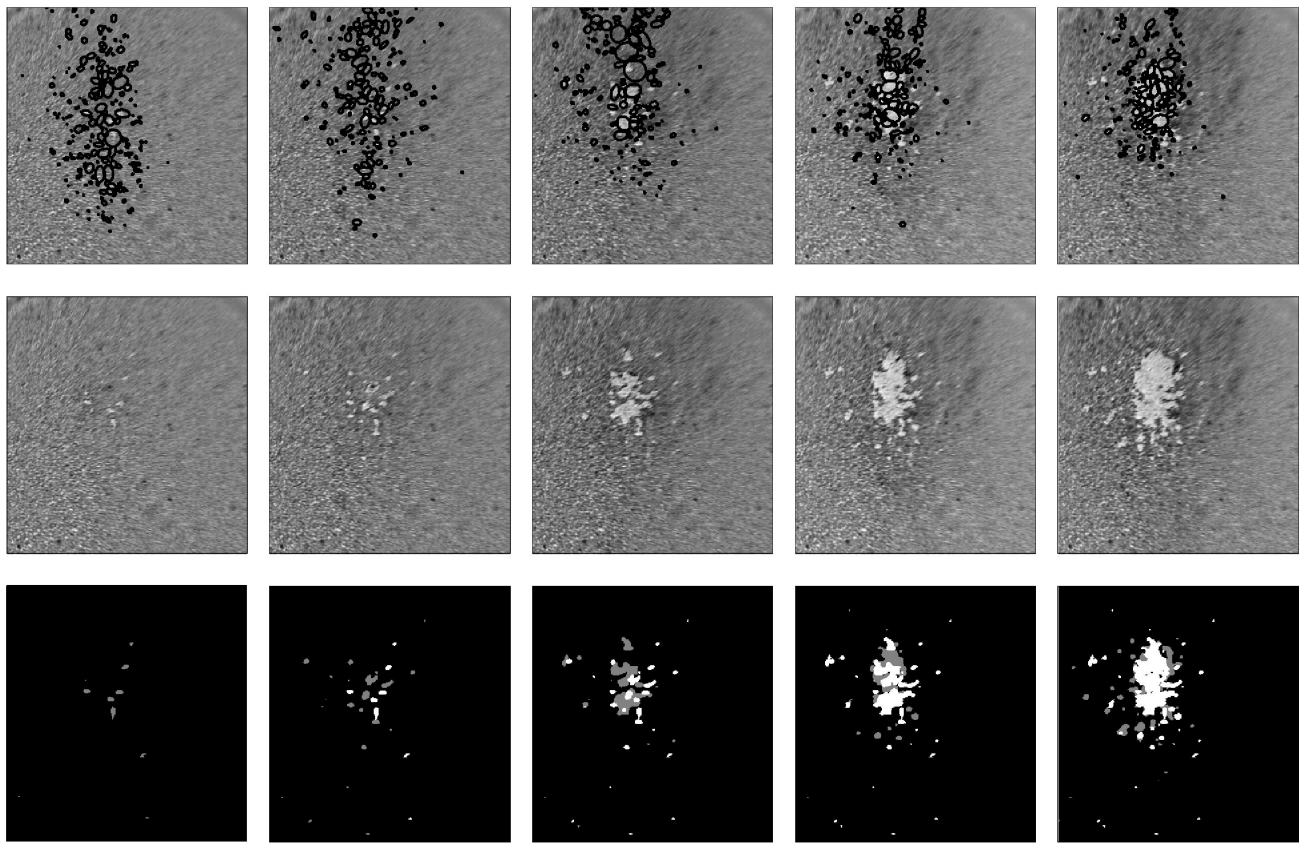

Figure 7.10: Visualization of cell detachment after one to five consecutive shock wave exposures from the image sequence of Fig. 7.9, The images are taken $10 \mathrm{~s}$ after shock wave application. In the upper row the outline of cavitation bubbles generated after the pulse are superimposed on the image. The last row depicts areas of new (gray) and previous (white) cell detachment. $3.0 \times 3.2 \mathrm{~cm}^{2}$.

sequence belongs to one shock wave exposure. From left to right the images display the cell layer $10 \mathrm{~ms}$ before, during, and $10 \mathrm{~ms}$ after cavitation generation. The number of the corresponding shock wave exposure is given in the left frame of every sequence. Areas where cells are detached from the substrate appear bright in the images. Always the second image of each sequence shows the cavitation cloud, which causes cell detachment. Not every bubble of the cloud induces cell detachment but all detachment sites can be correlated to bubble activity.

The cell detachment can be quantified using image subtraction and threshold separation methods. The exact image editing procedure is described in the paper by Junge et al. [143]. Figure 7.10 shows part of the edited images taken from the same recorded sequence of Fig. 7.9. The center row depicts background corrected images $10 \mathrm{~s}$ after one to five successive shock wave exposures from left to right. In the top row the outlines of the cavitation bubbles are superimposed and in the bottom row threshold separated images are given to better visualize areas where cells have been detached. In the last row areas 


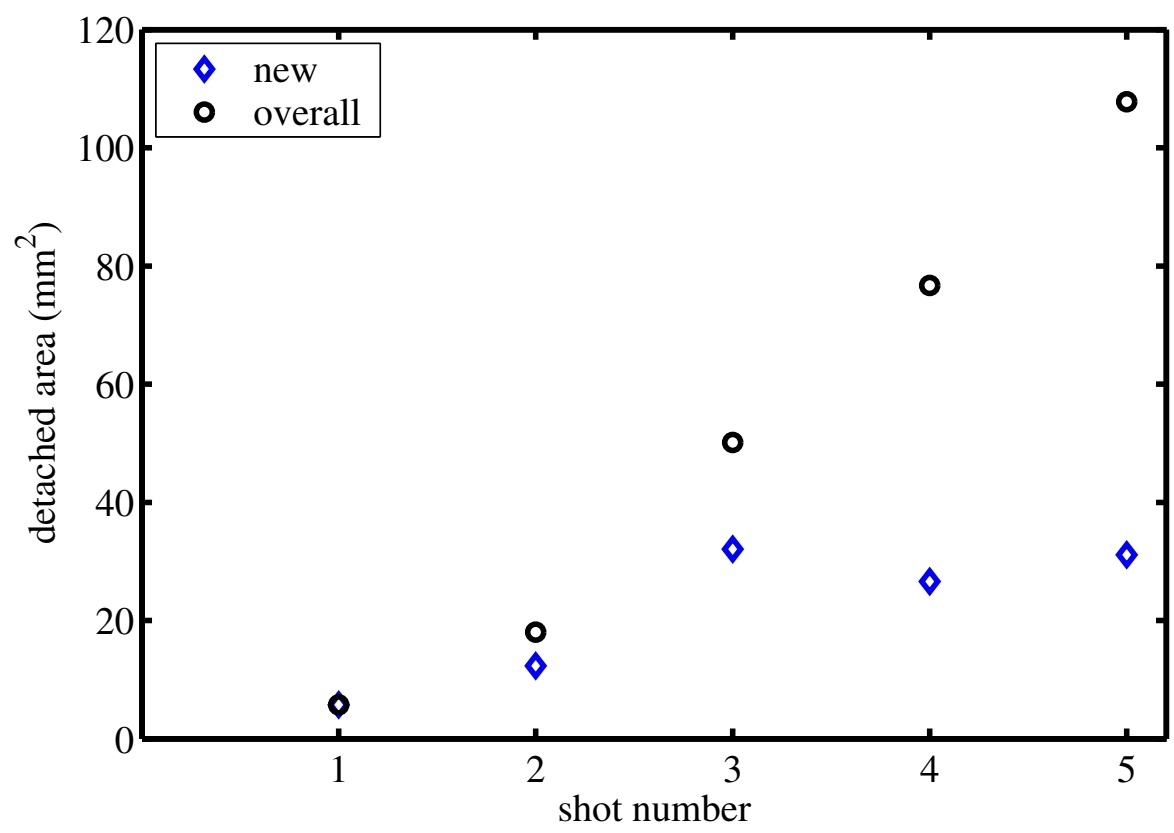

Figure 7.11: Area of cell detachment after one to five successive shock wave applications. Diamond markers indicate the new area of cell detachment after the previous shot. The circles show the total area of cell detachment.

of new cell detachment are depicted gray, while areas of previous cell detachment are depicted white. It is clearly visible that detachment is facilitated by previous shock wave applications. After the first two shots the cell layer is still densely packed but exhibits small circular regions where cells have been detached. Subsequent shock wave applications lead to a larger area of detachment in the center of the cavitation cloud. Small circular vacated regions are distributed around this center detachment area. In Fig. 7.11 the newly and the overall detached area is plotted against the number of shock wave applications. Up to three shots there is a steep rise of newly detached cells. For higher shock wave numbers the curve saturates, since most of the cells in the center of the bubble cloud have already been detached.

The facilitation of cell detachment can be used to perform large scale detachment. By moving the cell flask a few millimeter after each shock wave exposure almost the whole flask could be cleared of cells with less than 30 shock wave exposures as shown in Figs. 7.12 and 7.13 . The cavitation cloud seems to act as a cell brush sweeping adherent cells from the substrate. Depending on the speed of the translation stage the detachment procedure may take less than a minute. Of course the cells are subjected to significant 


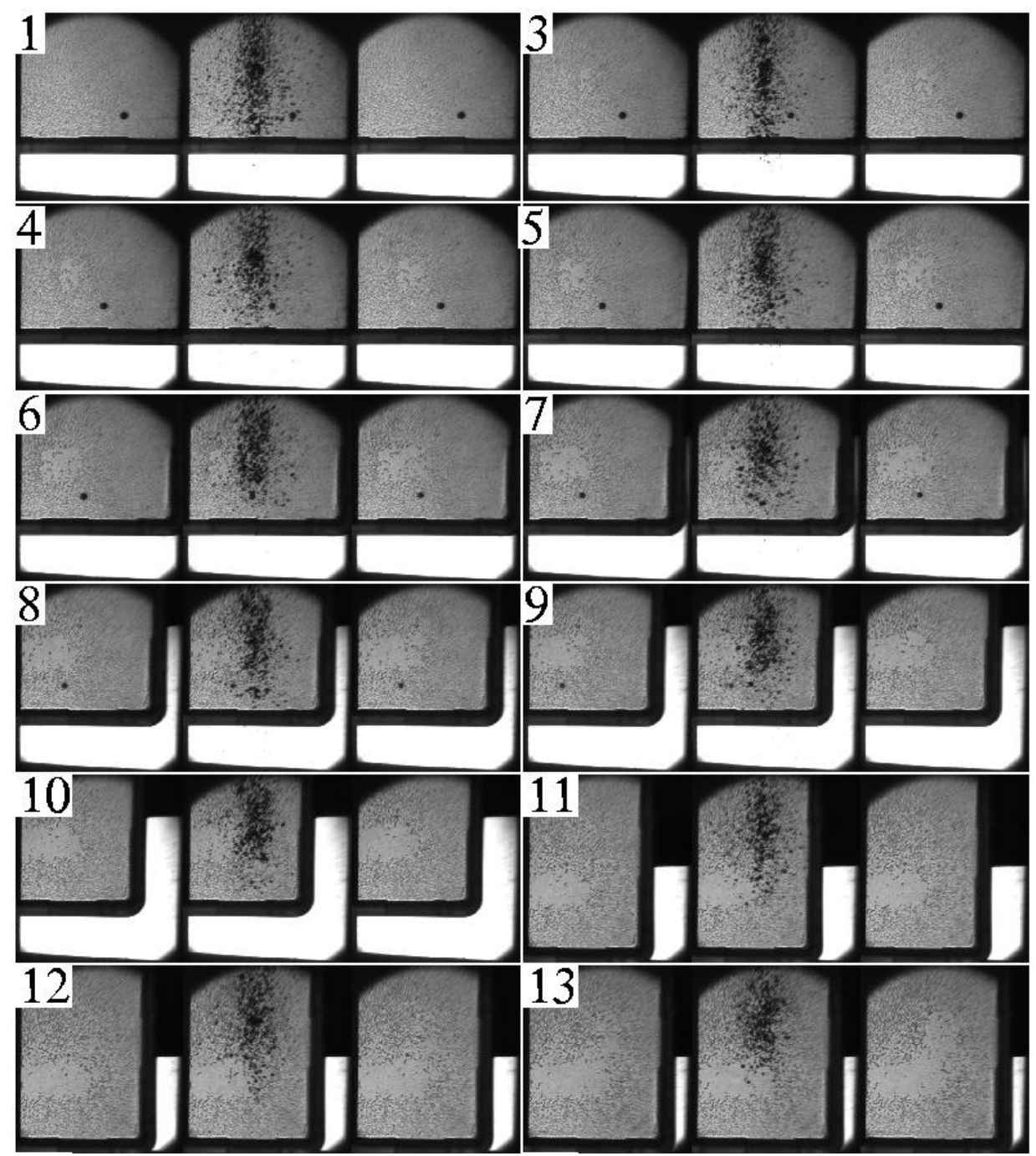

Figure 7.12: Large scale cell detachment after multiple shock wave applications and repositioning of the flask. Always three images belong to one shock wave exposure. Shock wave numbers are indicated in the pictures. Images are taken $10 \mathrm{~ms}$ before, during and $10 \mathrm{~ms}$ after cavitation generation from left to right. Time difference between the frames of a sequence is $10 \mathrm{~ms}$ and a single frame size covers $3.4 \times 3.9 \mathrm{~cm}^{2}$. 


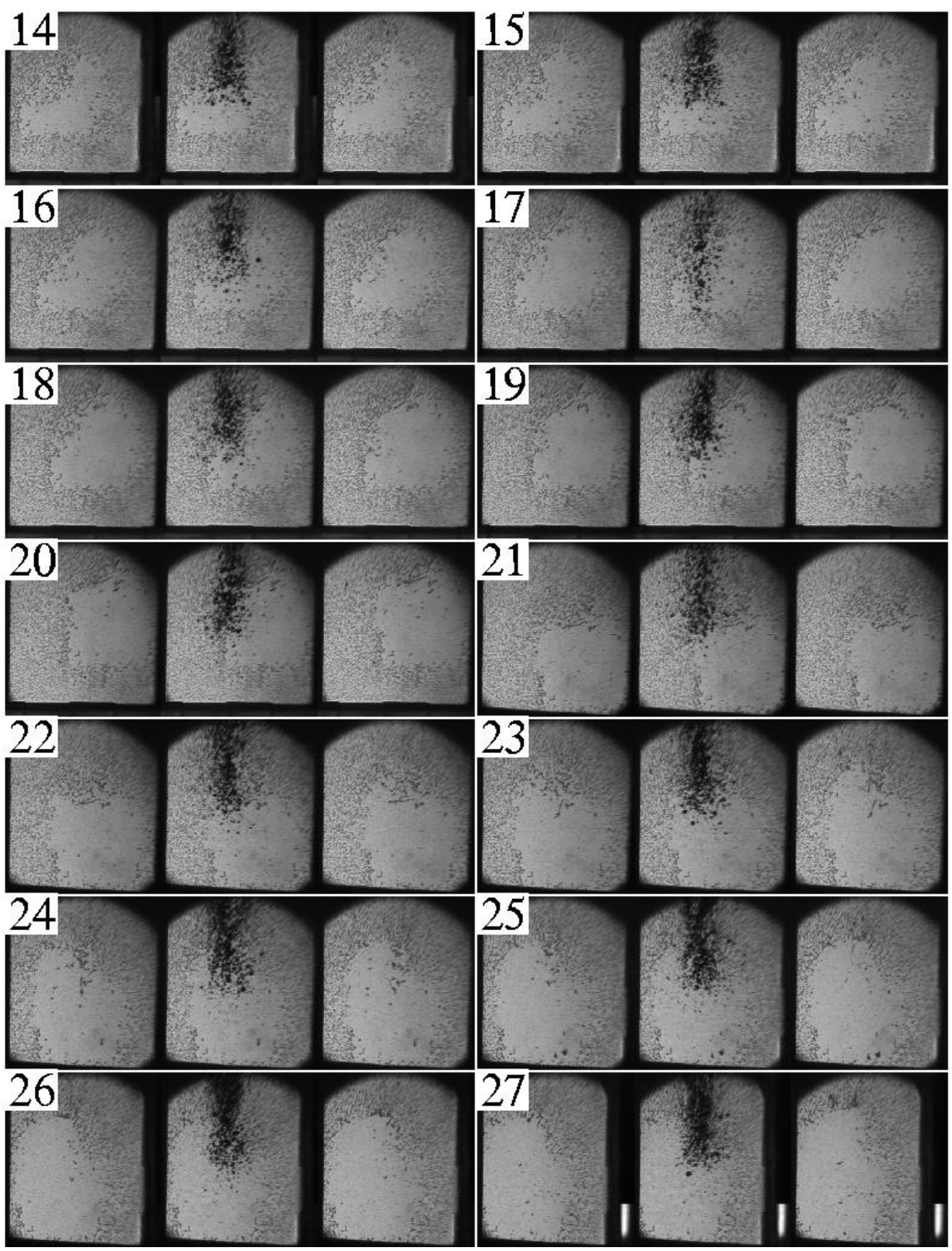

Figure 7.13: Continuation of the large scale cell detachment sequence of Fig. 7.12 

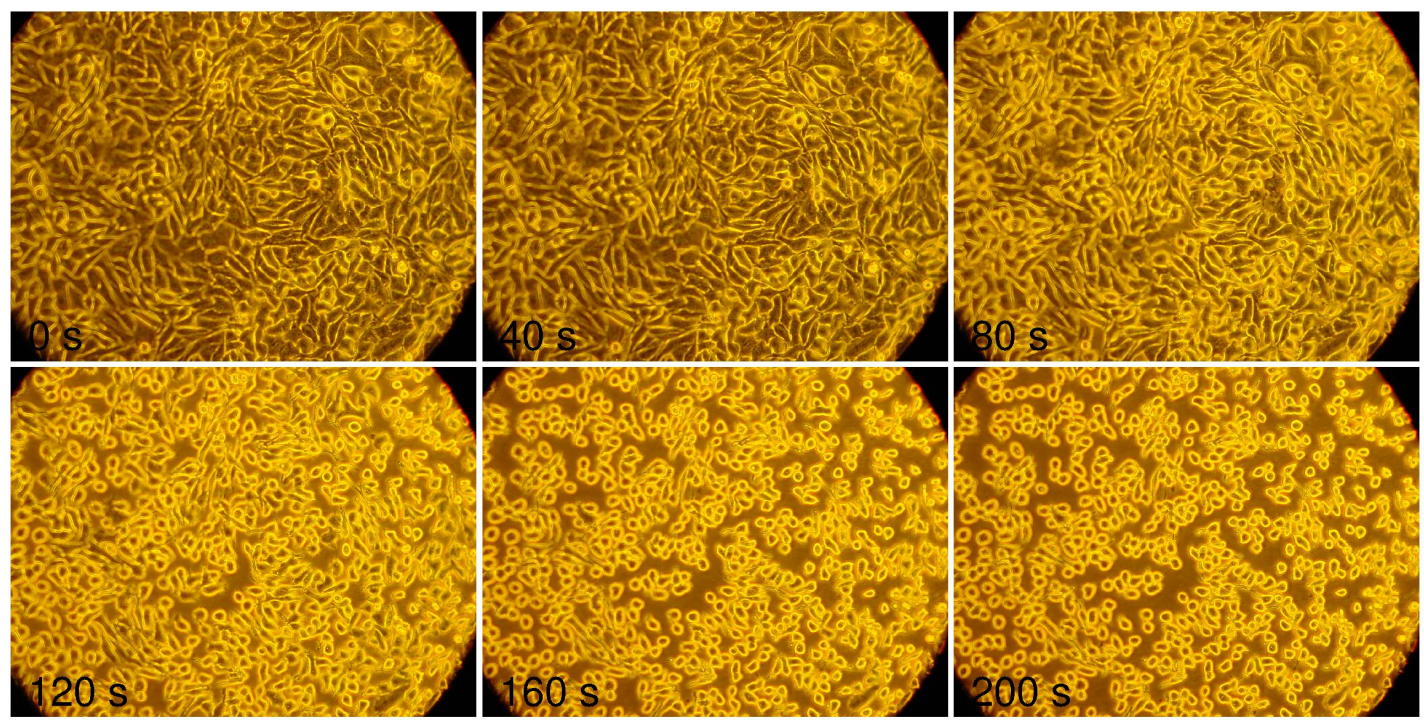

Figure 7.14: Cell detachment using standard trypsin cell culture procedure (trypsin $0.5 \mathrm{mg} / \mathrm{ml}$, EDTA $0.2 \mathrm{mg} / \mathrm{ml}$ in PBS). In the lower left corner of each frame the time with respect to trypsin application is given. A few minutes after trypsin treatment the cells round up and detach from the substrate.

shear stress during this process leading to transient and permanent cell permeabilization.

For comparison the detachment using the standard cell culture trypsin-EDTA procedure is shown in Fig. 7.14. After washing the cells twice with prewarmed PBS, $1 \mathrm{ml}$ trypsinEDTA has been added to the culture flask. The cells slowly detach from the substrate adopting a round shape during this process. Approximately 3 minutes after adding trypsin most of the cells are detached.

\subsection{Molecular uptake and cell viability}

We now turn the attention to transient and permanent membrane permeabilization by shock wave induced cavitation. Figure 7.15 depicts attached cells after shock wave exposure. Prior to shock wave treatment, the cell medium was supplemented with FITCdextran and treated for detection of molecular uptake and cell damage as described in Section 7.1.3. The substrate is partially cleared of cells caused by cavitation activity as shown in the previous Section 7.3. Cells, which have not been washed away but line the border between occupied and vacated regions, emit a strong green fluorescent light 


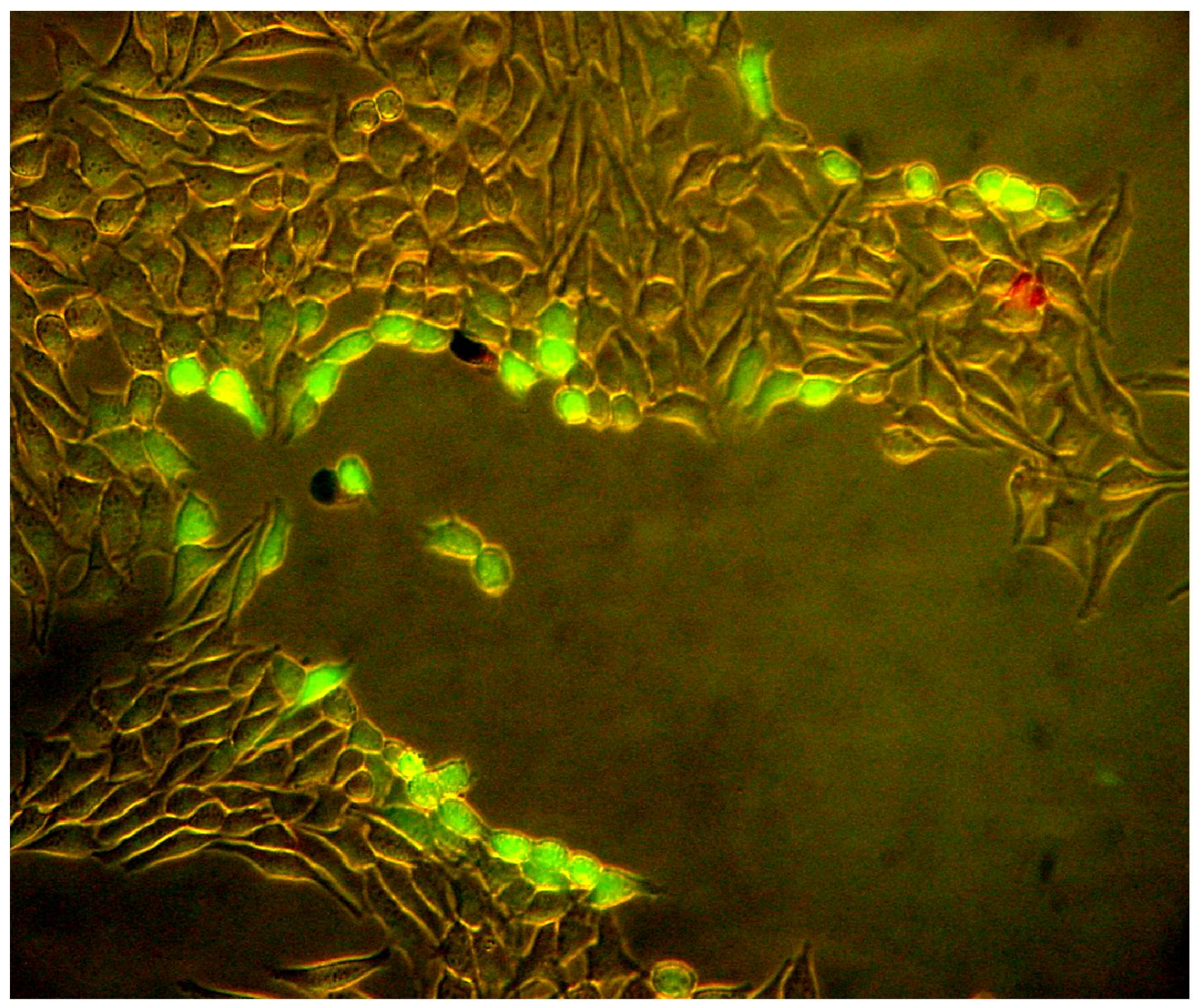

Figure 7.15: The image shows cells after shock wave exposure under a fluorescence microscope. Cells displaying green color have taken up FITC-dextran from the extracellular medium. Red color reveals dead cells because of the intercalation of ethidium bromide into the cell's DNA and RNA. The frame size is $0.72 \times 0.60 \mathrm{~mm}$. 


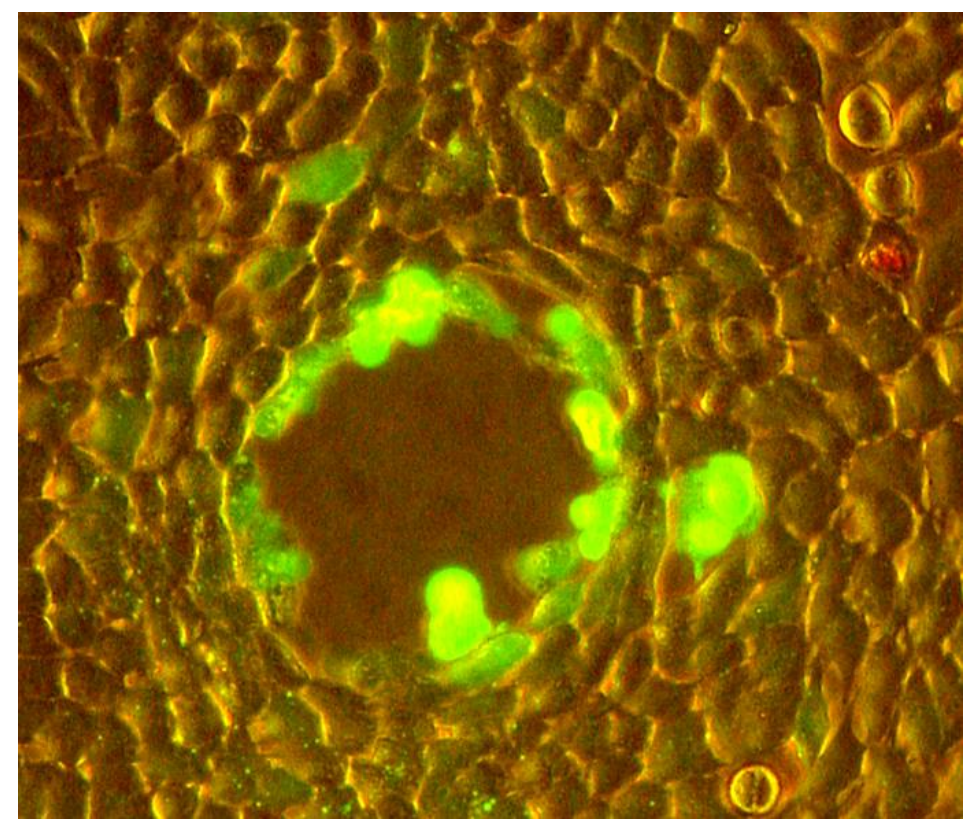

Figure 7.16: The image shows cells after shock wave exposure and under a fluorescence microscope. Cells displaying green color have taken up FITC-dextran from the extracellular medium. Red color reveals dead cells because of the intercalation of ethidium bromide into the DNA/RNA. The frame size is $0.38 \times 0.32 \mathrm{~mm}^{2}$.

upon excitation originating from the uptake of FITC-dextran. Only slight emission of green fluorescence is detected for cells further away from the region of cavitation-induced detachment. Dead cells appear red or brown in the image due to the intercalation of ethidium bromide into the DNA of permanently damaged cells. Almost no dead cells can be seen in Fig. 7.15, which is reasonable since most of the destroyed cells will round up and detach. Subsequently they are swept away during the washing procedure with PBS.

Figure 7.16 shows the FITC uptake around one of the small circular regions of cell detachment mentioned in Section 7.3. Again the uptake of FITC-dextran is mainly seen in cells surrounding the border of the cleared area. The result is a typical green ring-like structure seen all across the shock wave exposed cell layer.

Uptake of the smaller molecule calcein can be detected further away from the cavitation activity and cell detachment site than the uptake of FITC-dextran. An example is shown in Fig. 7.17, where calcein uptake is visible around two detachment sites at the upper right and the lower left of the image. The shape and size of the areas where detachment occurs are similar to those in the FITC-dextran experiments. However, calcein uptake occurs in many cells not directly in contact with the area of detachment. 


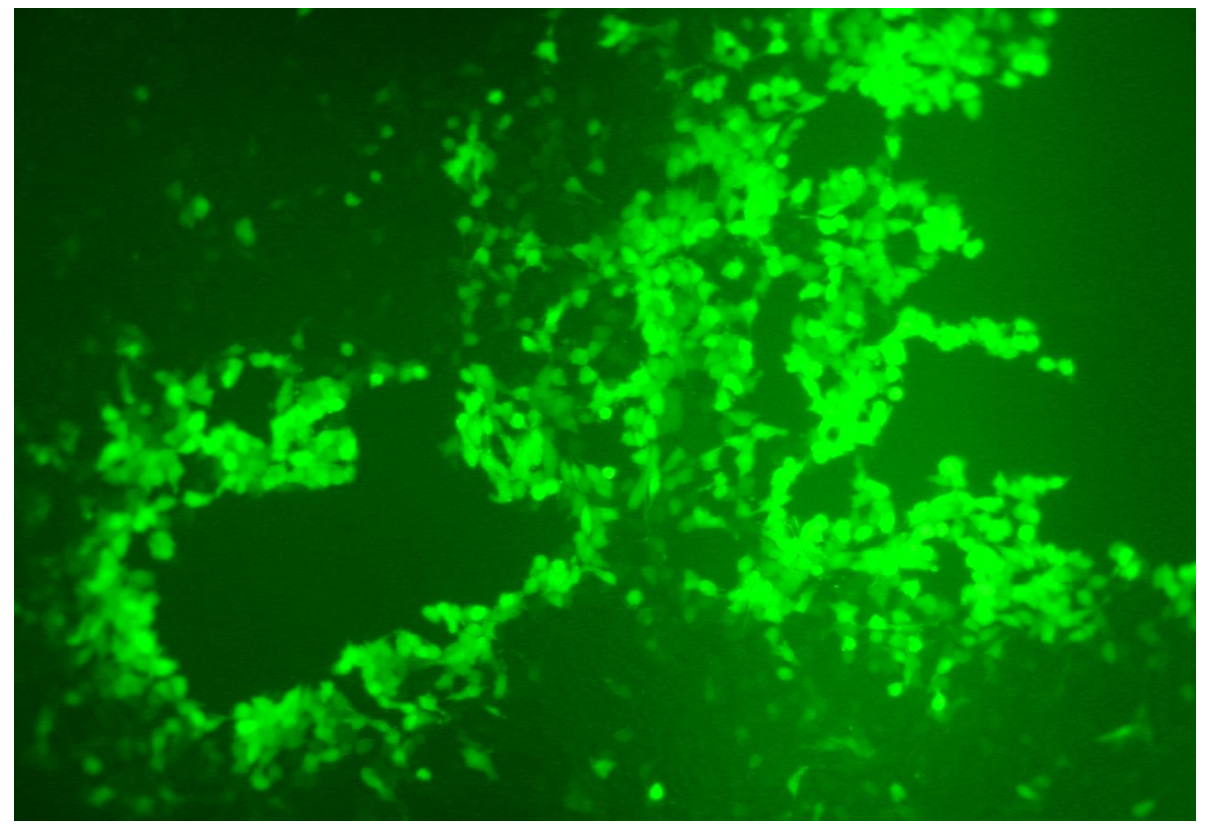

Figure 7.17: The image shows cells after shock wave exposure under a fluorescence microscope. Cells displaying green color have taken up calcein from the extracellular medium. The frame size is $1.45 \times 1.00 \mathrm{~mm}^{2}$.

Just as in the observations of cell detachment, no uptake of either FITC-dextran or calcein could be detected when the strength of the shock waves were below cavitation threshold.

The molecular uptake and viability of detached cells was checked after multiple shock wave applications with the lithotripter operating at $6 \mathrm{kV}$. Only one shot was taken at the same position and the flask was moved by $10 \mathrm{~mm}$ in the vertical or $2 \mathrm{~mm}$ in the horizontal position afterwards to detach cells from a large area as already shown in Figs. 7.12 and 7.13. Overall 341 cells were counted in three experiments carried out with the same setup. Almost $12 \%$ of the detached cells were permanently damaged as indicated by ethidium bromide staining. Only $11 \%$ of the surviving cells had taken up FITC-dextran. Culture growth of living cells after shock wave treatment has not been checked for this experiment. 

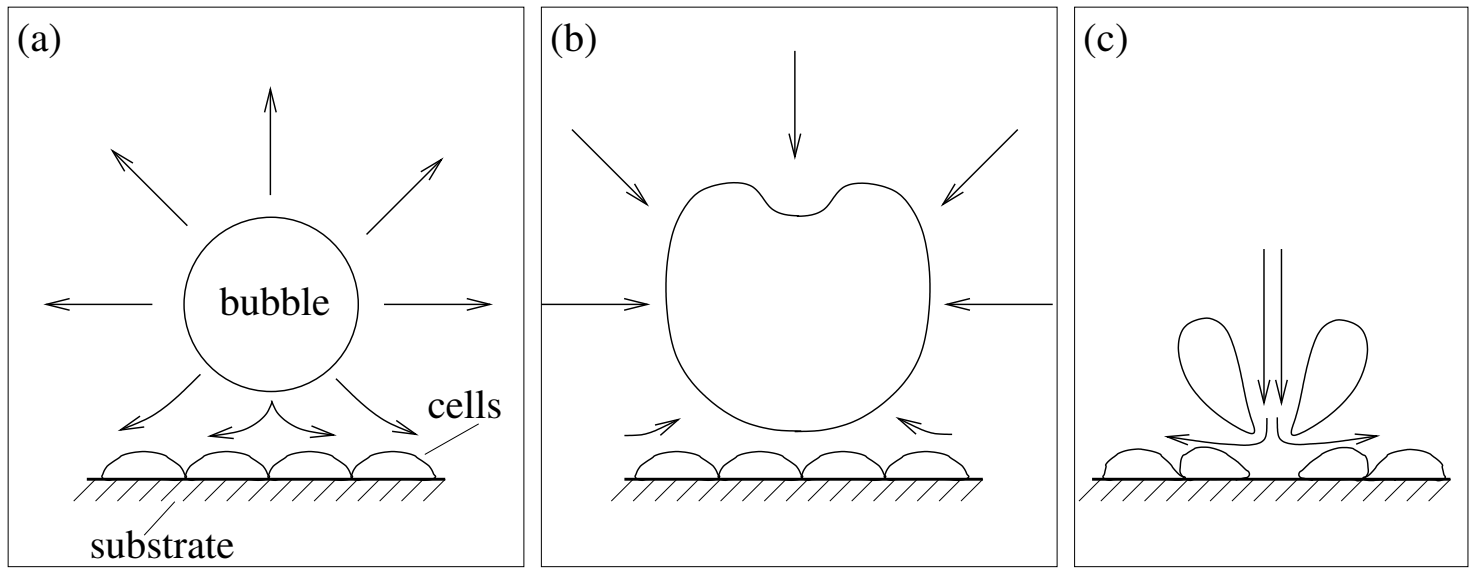

Figure 7.18: Schematic drawing of a bubble expanding and collapsing close to the cell substrate. a) The bubble expands and induces a radially outward directed shear flow at the substrate. b) During initial compression of the bubble the shear flow at the boundary is inverted and directed toward the projection of the bubble center. c) Jet-like collapse of the bubble. Cells are being detached from the boundary due to the strong outward directed shear flow.

\subsection{Discussion}

In the experiments presented in this chapter, cell detachment and molecular delivery are most probably caused by the effects of cavitation dynamics. This is supported by the absence of both, detachment of cells and molecular delivery, if the pressure amplitude remains below the threshold for cavitation generation. Furthermore, the positions of cell detachment sites were always in correlation with cavitation bubbles and in turn molecular uptake was always observed around the area of cell detachment. Other mechanisms like direct shearing by pressure gradients of the shock wave or the generation of surface waves seem to be of minor importance in this setup. The cavitation bubbles incept at impurities during the tensile pressure pulse following the shock wave. In a simple model, depicted in Fig. 7.18 we can separate the bubble dynamics in three phases: (1) First, there is an initial growth of the bubble after cavitation inception. The liquid surrounding the bubble will be forced outward from the bubble center. At the rigid boundary of the substrate, the flow will be redirected resulting in an outward radial streaming along the substrate with its center located at the projection of the bubble center onto the substrate. (2) At the beginning of bubble collapse the situation is reversed. Liquid flow will be directed toward the center of the bubble resulting in an inward streaming along the substrate. However, 
due to the disturbance of the flow caused by the rigid boundary the bubble collapse will not be spherical. (3) Upon final collapse the bubble will form a jet towards the substrate as we have already seen for smaller collapsing bubbles in Chapter 4. The aspherical collapse induces again an outward radial streaming along the substrate surface [103]. As discussed before in Chapter 4 and 5, the bubble behavior becomes more complicated if bubble-bubble interactions are taken into account. During all phases of bubble activity, the cells on the substrate will be subjected to shear stress. However, it is probable that the jet-induced flow generates the dominant shear stress, which is sufficiently strong to break the adherent forces of the cells in its vicinity. As a result of this flow-induced detachment, a roughly circular vacated region appears on the substrate. Cells, which are not detached but line the border of vacated regions, are subjected to a stress caused by the flow field. The stress exerted on the cells will decrease with increasing distance to the bubble collapse center. Depending on the strength of the stress, membranes may be transiently ruptured facilitating the uptake of molecules.

It was observed that in contrast to FITC-dextran the uptake of calcein takes place at larger distances from the center of cell detachment. This indicates that even further away from the center of bubble activity, some permeabilization of membranes is induced. However, either number or size of the pores induced by the weaker flow field is too small or their life span is too short to allow for detectable uptake of FITC-dextran. The rapid breaking of cadherin cell-cell adhesion bonds of those cells lining the border of vacated regions might also contribute to stronger perforation enabling the uptake of larger and more molecules.

The FITC-dextran uptake of detached cells after multiple shock wave exposure is very low (11\% of the surviving cells) compared to FITC-dextran uptake of attached cells lining the border of vacated regions after single shock wave application (usually more than 50\%). One might expect that cells, which are detached, have been subjected to an even stronger shear stress and consequently, should either be transiently permeabilized or destroyed. However, in total only $20 \%$ of the detached cells were permanently or transiently permeabilized. One reason of the relatively low membrane penetration of detached cells might be ascribed to an importance of cell-cell adhesion breaking for this process. If cells are detached in layers, not necessarily many cell-cell bonds are broken upon cell detachment. However, although cell-cell adhesion breaking might contribute to cell permeabilization it is not a mandatory precondition, since we have seen that some cells in the attached layer also show uptake of FITC-dextran. Furthermore, one would assume that violent cell-substrate debonding should also contribute to membrane perforation and molecular 
uptake. The main reason for the low membrane poration of detached cells has to be seen in the large scale detachment procedure. It was shown that cell detachment could be facilitated by previous shock wave exposure in neighboring regions. This was probably caused by subsequent breaking of adhesion points between cells and the substrate near the cavitation activity site. Therefore, it is possible that many of the detached cells have not been exposed to the threshold shear stress necessary to generate sufficient pores for molecular delivery. It is also possible that the mechanical stimulus induces a reaction inside the cells, which will trigger the weakening of adhesion forces allowing for large-scale detachment [61].

\subsection{Conclusion}

In this chapter it was shown with high-speed optical and fluorescence techniques that cell detachment and molecular uptake of adherent HeLa cells after shock wave application is caused by activity of cavitation bubbles. Bubbles incept during the trailing tensile pressure pulse of the lithotripter shock wave. They generate a flow field, which induces shear stress on the adherent cells in its vicinity. The shear flow is sufficiently strong to break adhesion forces between cells and substrate, destroy cells, or transiently permeabilize cell membranes facilitating molecular uptake. It is most likely that the strong shear generated during bubble collapse and jet impact is responsible for the observed effects. 


\section{Chapter 8}

\section{Outlook}

Today several medical applications exist, which rely on the use of ultrasound or shock waves. It is therefore important to assess the possibilities of cavitation generation during those applications and evaluate their potential beneficial or unfavorable side effects. For example, diagnostic ultrasound is a well established imaging technique in medicine and the use of coated microbubbles as contrast agents has further improved the diagnostic yield of this method [144]. The potential risks of cavitation damage to a patient depending on acoustic parameters have still to be evaluated and balanced against the gains achieved from ultrasound imaging. This may help to provide a safe set of operation parameters for efficient acoustic imaging with contrast agent bubbles.

New medical techniques based on cavitation or shock wave induced drug delivery may prove successful in the future. Various approaches including cancer and gene therapy are currently under investigation [58]. In applications where cell transfection is required it is important that genetic material can be introduced into the cell without compromising permanent damage to the target. The accomplishment of this goal may well depend on acoustic parameters during irradiation, the size and distribution of cavitation nuclei, and the region of interest, which is to be targeted. These parameters influence the dynamics of cavitation bubbles and their effect on the surrounding tissue. Further experiments on cavitation induced drug delivery under in vivo or similar conditions will hopefully provide clues on how to best approach gene transfection in therapy.

A still unsolved problem in specific drug delivery applications based on cavitation is how to control the position of bubbles and drugs with respect to the target cells. Evidently, viral based techniques are in this respect advantageous because ligands on the virus may bind to specific receptors on the target cell [145]. A primary goal is therefore to design acoustically excitable cavitation nuclei containing ligands that can also bind to receptors 
on the cell type to be targeted. It can be expected that a close proximity between cells and microbubbles lowers the pressure amplitudes and concentration of cavitation nuclei needed for sufficient membrane permeabilization. If it is further possible to incorporate the molecules to be delivered in the cavitation nucleus the drug concentration in the system could probably also be reduced. Another matter which should be addressed is the stability of the cavitation nuclei. If administered systematically they need to be stable at least as long as it takes them to reach the region of interest. To solve this problem designers of new cavitation nuclei may revert to knowledge gained from building ultrasound contrast agents, where microbubbles are stabilized by appropriate shells and by using low soluble gases inside the bubbles [146].

If the problems mentioned above can be solved, cavitation induced drug delivery promises to become a valuable technique for targeted drug administration in therapy. An advantage of microbubble carriers is that they can be localized using conventional ultrasonic imaging techniques. After reaching the target area a shock wave or an ultrasonic pulse of larger amplitude can be used to rupture the bubbles and release the drug in a desired region confined by the focal spot of the acoustic wave. 


\section{Acknowledgement}

During my $\mathrm{PhD}$ time I have worked with different groups all of whom I would now like to thank for helping me in one way or another:

First of all I want to thank Prof. Dr. W. Lauterborn for giving me the opportunity to do my PhD in the nice atmosphere of the Third Physical Institute at the University of Göttingen and supporting me during the different projects. From his group I would like to thank especially Dr. Robert Mettin and Dr. Thomas Kurz for their helpful support, reviewing the thesis, and the good suggestions during my PhD time. I would also like to thank Prof. Dr. D. Ronneberger, Prof. Dr. Ulrich Parlitz, Dr. U. Kaatze, and their respective group members for advice whenever needed. The tips from David Engster, Jörg Dittmar and Alexander Hornstein often saved me a lot of time during programming tasks. Further I thank Ulrike Schulz and Kerstin von Rhoden for help in the cell culture and Prof. Dr. Manfred Radmacher and Dr. Monica Fritz for loan of equipment. Also I want to thank the mechanical and electrical engineering groups with D. Hille and Dr. K. Lautscham for building desired gadgetry.

From the Physics of Fluids group in Twente I am especially grateful to Dr. Claus-Dieter Ohl for making it possible to work with him, Manish Aurora, Roy Ikink, and Dr. Lutz Junge on the detachment and permeabilization of cells and for his helpful suggestions ever after. I would also like to thank Yvonne Kramer for providing us with cells.

Further I want to thank my brother Dr. Christian Wolfrum from the Laboratory of Metabolic Diseases of the Rockefeller University in New York, who taught me several biochemical techniques and how to work with cells in the first place.

From the forensic department of the University of Göttingen I would like to thank Bernward Sigmund and Dr. H. Kijewski who invited Robert and me to join them on their ballistic experiments.

Most of all I am grateful to my parents Hildegard and Dr. Rüdiger Wolfrum and my 
wife Silke Wolfrum who stayed with me during my whole course of studies. Thank you for your support throughout. 


\section{Bibliography}

[1] Sir J. Thornycroft, S.W. Barnaby. Torpedo Boat Destroyers. Minutes of Proc. Inst. of Civil Engineers, 122 (1895) 51-103.

[2] I.N. Kirschner, B.J. Rosenthal, J.S. Uhlman. Simplified dynmical systems analysis of supercavitating high-speed bodies. In: Proceedings of the 5th Int. Symposium on Cavitation - CAV2003 (Osaka), 2003.

[3] D.F. Gaitan, L.A. Crum, C.C. Church, R.A. Roy. Sonoluminescence and bubble dynamics for a single, stable, cavitation bubble. J. Acoust. Soc. Am., 91 (1992) 3166-3183.

[4] S. Hilgenfeldt, D. Lohse. Predictions for Upscaling Sonoluminescence. Phys. Rev. Lett., 82 (1999) 1036-1039.

[5] W.C. Moss, D.B. Clarke, D.A. Young. Calculated pulse width and spectra of a single sonoluminescing bubble. Science, 276 (1997) 1187-1190.

[6] D. Schanz, T. Kurz, B. Metten, W. Lauterborn. Molekulardynamische Untersuchung der Kollapsdynamik von Sonolumineszenzblasen. In: M. Vorländer, Ed., Fortschritte der Akustik - DAGA'03 (Aachen), 2003, 834-835.

[7] F. Lepoint-Mullie, D. De Pauw, T. Lepoint, P. Supiot, R. Avni. Nature of extreme conditions in single sonoluminescing bubbles. J. Phys. Chem., 100 (1996) 12138 12141.

[8] K.S. Suslick. Sonochemistry. Science, 247 (1990) 1439-1444.

[9] R.T. Knapp, J.W. Daily, F.G. Hammit. Cavitation. McGraw-Hill, New York, 1970.

[10] F.R. Young. Cavitation. McGraw-Hill, London, 1989.

[11] T.G. Leighton. The Acoustic Bubble. Academic Press, London, 1994. 
[12] C.E. Brennen. Cavitation and bubble dynamics. Oxford University Press, Oxford, 1995.

[13] Lord Rayleigh. On the pressure developed in a liquid during the collapse of a spherical cavity. Phil. Mag., 34 (1917) 94-98.

[14] W. Lauterborn. High-speed photography of laser-induced breakdown in liquids. Appl. Phys. Lett., 21 (1972) 27-29.

[15] A. Vogel, W. Lauterborn. Time-resolved particle image velocimetry used in the investigation of cavitation bubble dynamics. Appl. Optics, 29 (1988) 1869-1876.

[16] A. Vogel, W. Lauterborn, R. Timm. Optical and acoustic investigations of the dynamics of laser-produced cavitation bubbles near a solid boundary. J. Fluid Mech., 206 (1989) 299-338.

[17] A. Philipp, M. Delius, C. Scheffczyk, A. Vogel, W. Lauterborn. Interaction of lithotripter-generated shock waves with air bubbles. J. Acoust. Soc. Am., 93 (1993) 2496-2509.

[18] W. Lauterborn, T. Kurz, R. Mettin, C.-D. Ohl. Experimental and theoretical bubble dynamics. Adv. Chem. Phys., 110 (1999) 295-379.

[19] A. Vogel, W. Hentschel, J. Hozfuss, W. Lauterborn. Cavitation bubble dynamics and acoustic transient generation in ocular surgery with pulsed Neodymium:YAG lasers. Ophthalmology, 93 (1986) 1259-1269.

[20] K. Sellier, B.P. Kneubuehl. Wundballistik und ihre ballistischen Grundlagen. Springer, Berlin, 2001.

[21] A.D. Miller. Human gene therapy comes of age. Nature, 357 (1992) 455-460.

[22] P.L. Felgner, G. Rhodes. Gene therapeutics. Nature, 349 (1991) 351-352.

[23] P.L. Felgner, G.M. Ringold. Cationic liposome-mediated transfection. Nature, 337 (1989) 387-388.

[24] I. Koltover, T. Salditt, J. Rädler, C.R. Safinya. An Inverted Hexagonal Phase of DNA-Cationic Liposome Complexes Related to DNA Release. Science, 281 (1998) $78-81$. 
[25] N. Oudrhiri, J.-P. Vigneron, M. Peuchmaur, T. Leclerc, J.-M. Lehn, P. Lehn. Gene transfer by guanidinium-cholesterol cationic lipids into airway epithelial cells in vitro and in vivo. Proc. Natl. Acad. Sci. USA, 68 (1997) 1864-1870.

[26] D. Luo, W.M. Saltzman. Synthetic DNA delivery systems. Nat. Biotechnol., 18 (2000) 33-37.

[27] T. Tsong. Electroporation of cell membranes. Biophys. J., 60 (1991) 297-306.

[28] M.R. Prausnitz, J.D. Corbett, J.A. Gimm, D.E. Golan, R. Langer, J.C. Weaver. Millisecond measurement of transport during and after an electroporation pulse. Biophys. J., 68 (1995) 1864-1870.

[29] J.A. Lundqvist, F. Sahlin, M.A.I. Aberg, A. Strömberg, P.S. Eriksson, O. Orwar. Altering the biochemical state of individual cultured cells and organelles with ultramicroelectrodes. Proc. Natl. Acad. Sci. USA, 95 (1998) 10356-10360.

[30] T.M. Klein, E.D. Wolf, R. Wu, J.C. Sanford. High-velocity microprojectiles for delivering nucleic acids into living cells. Nature, 327 (1987) 70-73.

[31] E. Helenius, M. Boije, V. Niklander-Terri, E.T. Palva, T.H. Teeri. Gene delivery into intact plants using the Helios gene gun. Plant Mol. Biol. Rep., 18 (2000) 287a-287l.

[32] U.K. Tirlapur, K. König. Targeted transfection by femtosecond laser. Nature, 418 (2002) 290-291.

[33] J.S. Soughayer, T. Krasieva, S.C. Jacobson, J.M. Ramsey, B.J. Tromberg, N.L. Allbritton. Characterization of cellular optoporation with distance. Anal. Chem., 72 (2000) 1342-1347.

[34] A.G. Doukas, T.J. Flotte. Physical characteristics and biological effects of laserinduced stress waves. Ultrasound Med. Biol., 22 (1996) 151-164.

[35] S. Lee, T. Anderson, H. Zhang, T.J. Flotte, A.G. Doukas. Alteration of cell membrane by stress waves in vitro. Ultrasound Med. Biol., 22 (1996) 1285-1293.

[36] S. Lee, D.J. McAuliffe, H. Zhang, Z. Xu, J. Taitelbaum, T.J. Flotte, A.G. Doukas. Stress-wave-induced membrane permeation of red blood cells is facilitated by aquaporins. Ultrasound Med. Biol., 23 (1997) 1089-1094. 
[37] S. Gambihler, M. Delius, J.W. Ellwart. Permeabilization of the plasma membrane of L1210 mouse leukemia cells using lithotripter shock waves. J. Membrane Biol., 141 (1994) 267-275.

[38] S. Bao, B. D. Thrall, D. L. Miller. Transfection of a reporter plasmid into cultured cells by sonoporation in vitro. Ultrasound Med. Biol., 23 (1997) 953-959.

[39] M. Delius, P.-H. Hofschneider, U. Lauer, K. Messmer. Extracorporeal shock waves for gene therapy? Lancet, 345 (1995) 1377.

[40] J.W.J. Greenleaf, M.E. Bolander, G. Sarkar, M.B. Goldring, J.F. Greenleaf. Artificial cavitation nuclei significantly enhance acoustically induced cell transfection. Ultrasound Med. Biol., 24 (1998) 587-595.

[41] A.A. Brayman, M.L. Coppage, S. Vaidya, M.W. Miller. Transient poration and cell surface receptor removal from human lymphocytes in vitro. Ultrasound Med. Biol., 25 (1999) 999-1008.

[42] P.E. Huber, J. Jenne, J. Debus, M.F. Wannenmacher, P. Pfisterer. A comparison of shock wave and sinusoidal-focused ultrasound-induced localized transfection of HeLa cells. Ultrasound Med. Biol., 25 (1999) 1451-1457.

[43] D.L. Miller, S. Bao, J.E. Morris. Sonoporation of cultured cells in the rotating tube exposure system. Ultrasound Med. Biol., 25 (1999) 143-149.

[44] Z. Qian, R. D. Sagers, W. G. Pitt. Investigation of the mechanism of the bioacoustic effect. J. Biomed. Mater. Res., 44 (1999) 198-205.

[45] M. Ward, J. Wu, J.-F. Chiu. Ultrasound-induced cell lysis and sonoporation enhanced by contrast agents. J. Acoust. Soc. Am., 105 (1999) 2951-2967.

[46] P. Zhong, H. Lin, X. Xi, S. Zhu, E.S. Bhogte. Shock wave-inertial microbubble interaction: Methodology, physical characterization, and bioeffect study. J. Acoust. Soc. Am., 105 (1999) 1997-2009.

[47] S. Koch, P. Pohl, U. Cobet, N.G. Rainov. Ultrasound enhancement of liposomemediated cell transfection is caused by cavitation effects. Ultrasound Med. Biol., 26 (2000) 897-903.

[48] T. Kodama, M.R. Hamblin, A.G. Doukas. Cytoplasmic molecular delivery with shock waves: Importance of impulse. Biophys. J., 26 (2000) 897-903. 
[49] D.L. Miller, J. Quddus. Sonoporation of monolayer cells by diagnostic ultrasound activation of contrast-agent gas bodies. Ultrasound Med. Biol., 26 (2000) 661-667.

[50] M. Ward, J. Wu, J.-F. Chiu. Experimental study of the effects of optison concentration on sonoporation in vitro. Ultrasound Med. Biol., 26 (2000) 1169-1175.

[51] H.R. Guzmán, D.X. Nguyen, S. Khan, M.R. Prausnitz. Ultrasound-mediated disruption of cell membranes. I. Quantification of molecular uptake and cell viability. J. Acoust. Soc. Am., 110 (2001) 588-596.

[52] K. Tschoep, G. Hartmann, R. Jox, S. Thompson, A. Eigler, A. Krug, S. Erhardt, G. Adams, S. Endres, M. Delius. Shock waves: a novel method for cytoplasmic delivery of antisense oligonucleotides. J. Mol. Med., 79 (2001) 306-313.

[53] J.P. Ross, X. Cai, J.-F. Chiu, J. Yang, J. Wu. Optical and atomic force microscopic studies on sonoporation. J. Acoust. Soc. Am., 111 (2002) 1161-1164.

[54] J. Wu, J.P. Ross, J.-F. Chiu. Reparable sonoporation generated by microstreaming. J. Acoust. Soc. Am., 111 (2002) 1460-1464.

[55] D.L. Miller, S. Bao, R.A. Gies, B.D. Thrall. Ultrasonic enhancement of gene transfection in murine melanoma tumors. Ultrasound Med. Biol., 25 (1999) 1425-1430.

[56] D.L. Miller, J. Quddus. Diagnostic ultasound activation of contrast agent gas bodies induces capillary rupture in mice. Proc. Natl. Acad. Sci. USA, 26 (2000) 661-667.

[57] D.L. Miller, R.A. Gies. The influence of ultrasound frequency and gas-body composition on the contrast agent-mediated enhancement of vascular bioeffects in mouse intestine. Ultrasound Med. Biol., 26 (2000) 307-313.

[58] M.W. Miller. Gene transfection and drug delivery. Ultrasound Med. Biol., 26 (2000) $59-62$.

[59] V.S. Teslenko, V.P. Nikolin, I.V. Mastikhin, N.G. Kolosova, V.N. Gorchakov. Tumor growth inhibition by local activation of cellular sensitivity to drugs by focused acoustic shock waves. In: Proceedings of the 16th Int. Symp. on Nonlinear Acoustics - ISNA16 (Moscow), 2002.

[60] B. Alberts, D. Bray, J. Lewis, M. Raff, K. Roberts, J.D. Watson. Molecular biology of the cell, 3rd ed. Garland Publishing, New York, 1994. 
[61] C. Zhu, G. Bao, N. Wang. Cell mechanics: Mechanical response, cell adhesion, and molecular deformation. Annu. Rev. Biomed. Eng., 2 (2000) 189-226.

[62] E. Décavé, D. Garrivier, Y. Bréchet, B. Fourcade, F. Bruckert. Shear flow-induced detachment kinetics of dictyostelium discoideum cells from solid substrate. Biophys. J., 82 (2002) 2383-2395.

[63] Y.B. Zel'dovich, Y.P. Raizer. Physics of shock waves and high-temperature hydrodynamic phenomena. Dover Publications, Mineola, 2002.

[64] R.H. Cole. Underwater explosions. Princeton University Press, Princeton, 1948.

[65] M. Delius. Lithotripsy. Ultrasound Med. Biol., 26 (2000) 55-58.

[66] M. Lokhandwalla, B. Sturtevant. Fracture mechanics model of stone comminution in ESWL and implications for tissue damage. Phys. Med. Biol., 45 (2000) 1923-1940.

[67] W. Eisenmenger. The mechanisms of stone fragmentation in ESWL. Ultrasound Med. Biol., 27 (2001) 683-693.

[68] X. Xi, P. Zhong. Dynamic photoelastic study of the transient stress fields in solids during shock wave lithotripsy. J. Acoust. Soc. Am., 109 (2001) 1226-1239.

[69] S. Zhu, F.H. Cocks, G.M., Preminger P.Zhong. The role of stress waves and cavitation in stone comminution in shock wave lithotripsy. Ultrasound Med. Biol., 28 (2002) 661-671.

[70] S.M. Gracewski, G. Dahake, Z. Ding, S.J. Burns, E.C. Everbach. Internal stress wave measurements in solids subjected to lithotripter pulses. J. Acoust. Soc. Am., 94 (1993) 652-661.

[71] G. Dahake, S.M. Gracewski. Finite difference predictions of P-SV wave propagation inside submerged solids. II. Effect of geometry. J. Acoust. Soc. Am., 102 (1997) $2125-2137$.

[72] C.C. Church. A theoretical study of cavitation generated by an extracorporeal shock wave lithotripter. J. Acoust. Soc. Am., 86 (1989) 215-227.

[73] A.J. Coleman, J.E. Saunders, L.A. Crum, M. Dyson. Acoustic cavitation generated by an extracorporeal shock wave lithotripter. Ultrasound Med. Biol., 13 (1987) $69-76$. 
[74] L.A. Crum. Cavitation microjets as a contributory mechanism for renal calculi disintegration in ESWL. J. Urology, 140 (1988) 1587-1590.

[75] W. Sass, M. Bräunlich, H.P. Dreyer, E. Matura, W. Folberth, H.G. Priesmeyer, J. Seifert. The mechanisms of stone disintegration by shock waves. Ultrasound Med. Biol., 17 (1991) 239-243.

[76] P. Zhong, C.J. Chuong, G.M. Preminger. Propagation of shock waves in elastic solids caused by cavitation microjet impact. II: Application in extracorporeal shock wave lithotripsy. J. Acoust. Soc. Am., 94 (1993) 29-36.

[77] K. Rink, G. Delacrétaz, G. Pittomvils, Boving R, J.P. Lafaut. Incidence of cavitation in the fragmentation process of extracorporeal shock wave lithotriptors. Appl. Phys. Lett., 64 (1994) 2596-2598.

[78] M. Delius. Minimal static excess pressure minimises the effect of extracorporeal shock waves on cells and reduces it on gallstones. Ultrasound Med. Biol., 23 (1997) 611-617.

[79] B.D. Storey, A.J. Szeri. Mixture segregation within sonoluminescence bubbles. J. Fluid Mech., 396 (1999) 203-221.

[80] M. Voß. Numerische, theoretische und experimentelle Untersuchungen zur Kavitationsblasendynamik. Dissertation, Georg-August-Universität zu Göttingen, 2002.

[81] M. Delius, R. Denk, C. Berding, H.-G. Liebich, M. Jordan, W. Brendel. Biological effects of shock waves: Cavitation by shock waves in piglet liver. Ultrasound Med. Biol., 16 (1990) 467-472.

[82] M. Delius. Medical applications and bioeffects of extracorporeal shock waves. Shock Waves, 4 (1994) 52-72.

[83] W.H. Besant. Hydrostatics and Hydrodynamics. Deighton Bell, Cambridge, 1859.

[84] M.S. Plesset. The dynamics of cavitation bubbles. J. Appl. Mech., 16 (1949) 277 282.

[85] B.E. Noltingk, E.A. Neppiras. Cavitation produced by ultrasonics. Proc. Phys. Soc. London B., 63 (1950) 674-685. 
[86] B.E. Noltingk, E.A. Neppiras. Cavitation produced by ultrasonics, theoretical conditions for the onset of cavitation. Proc. Phys. Soc. London B., 64 (1951) 1032-1038.

[87] H. Poritsky. The collapse or growth of a spherical bubble or cavity in a viscous fluid. Proc. of the 1st U.S. Nat. Congr. in Appl. Mech., (1952) 813-821.

[88] J.B. Keller, M. Miksis. Bubble oscillations of large amplitude. J. Acoust. Soc. Am., 68 (1980) 628-633.

[89] U. Parlitz, V. English, C. Scheffczyk, W. Lauterborn. Bifurcation structure of bubble oscillators. J. Acoust. Soc. Am., 88 (1990).

[90] F. R. Gilmore. Hydrodynamics laboratory report 26-4. Technical Report, California Institut of Technology, 1952.

[91] C. C. Wu, P. H. Roberts. Shock-wave propagation in a sonoluminescing gas bubble. Phys. Rev. Lett., 70 (1993) 3424-3427.

[92] L.A. Crum. Sonoluminescence, sonochemistry, and sonophysics. J. Acoust. Soc. Am., 95 (1994) 559-562.

[93] L. Kondić, J. I. Gersten, C. Yuan. Theoretical studies of sonoluminescence radiation: Radiative transfer and parametric dependence. Phys. Rev. E, 52 (1995) 4976-4990.

[94] V.Q. Vuong, A.J. Szeri. Sonoluminescence and diffusive transport. Physics of Fluids, 8 (1996) 2354-2364.

[95] D. Lohse, M. Brenner, T. Dupont, S. Hilgenfeldt, B.Johnston. Sonoluminescing air bubbles rectify argon. Phys. Rev. Lett., 78 (1997) 1359-1362.

[96] K. Yasui. Alternative model of single-bubble sonoluminescence. Phys. Rev. E, 56 (1997) 6750-6760.

[97] W. Lauterborn, H. Bolle. Experimental investigations of cavitation-bubble collapse in the neighbourhood of a solid boundary. J. Fluid Mech., 72 (1975) 391-399.

[98] A. Vogel, W. Lauterborn. Acoustic transient generation by laser-produced cavitation bubbles near solid boundaries. J. Acoust. Soc. Am., 84 (1988) 719-731.

[99] A. Philipp, W. Lauterborn. Damage of solid surfaces by single laser-produced cavitation bubbles. Acustica, 83 (1997) 223-227. 
[100] J.-C. Isselin, A.-P. Alloncle, M. Autric. On laser induced single bubble near a solid boundary: Contribution to the understanding of erosion phenomena. J. Appl. Phys., 84 (1998) 5766-5771.

[101] A. Philipp, W. Lauterborn. Cavitation errosion by single laser-produced bubbles. J. Fluid Mech., 361 (1998) 75-116.

[102] S.J. Shaw, T.P. Gentry W.P. Schiffers, D.C. Emmony. A study of the interaction of a laser-generated cavity with a nearby solid boundary. J. Phys. D: Appl. Phys., 32 (1999) 1612-1617.

[103] E.-A. Brujan, G.S. Keen, A. Vogel, J.R. Blake. The final stage of the collapse of a cavitation bubble close to a rigid boundary. Phys. Fluids, 14 (2002) 85-92.

[104] G.L. Chahine. Experimental and asymptotic study of nonspherical bubble collapse. Appl. Sci. Res., 38 (1982) 187-197.

[105] Y. Tomita, A. Shima, T. Ohno. Collapse of multiple gas bubbles by a shock wave and induced impulsive pressure. J. Appl. Phys., 56 (1984) 125-131.

[106] N. K. Bourne, J. E. Field. A high-speed photographic study of cavitation damage. J. Appl. Phys., 78 (1995) 4423-4427.

[107] C.D. Ohl. Cavitation inception following shock wave passage. Phys. Fluids, 14 (2002) 3512-3521.

[108] Olgert Lindau, Werner Lauterborn. Cinematographic observation of the collapse and rebound of a laser-produced cavitation bubble near a wall. J. Fluid Mech., $\mathbf{4 7 9}$ (2003) 327-348.

[109] G.L. Chahine. Oscillation and collapse of a cavitation bubble in the vicinity of a two-liquid interface. In: W. Lauterborn, Ed., Cavitation and inhomogeneities in underwater acoustics, 1980, 23-29.

[110] J.R. Blake, D.C. Gibson. Cavitation bubbles near boundaries. Ann. Rev. Fluid Mech., 19 (1987) 99-123.

[111] E.-A. Brujan, K. Nahen, P. Schmidt, A. Vogel. Dynamics of laser-induced cavitation bubbles near an elastic boundary used as a tissue phantom. Proceedings of the 15th Int. Symp. on Nonlinear Acoustics - ISNA15 (Göttingen 1999). AIP Conf. Proc., 524 (2000) 381-384. 
[112] J.R. Blake, B.B. Taib, G.Doherty. Transient cavities near boundaries. J. Fluid Mech., 170 (1986) 479-497.

[113] J.P. Best. The formation of toroidal bubbles upon the collapse of transient cavities. J. Fluid Mech., 251 (1903) 79-107.

[114] S. Zhang, J.H. Duncan. On the nonspherical collapse and rebound of a cavitation bubble. Phys. Fluids, 6 (1994) 2352-2362.

[115] Z. Ding, S.M. Cewski. The behaviour of gas cavity impacted by a weak or strong shock wave. J. Fluid Mech., 309 (1996) 183-209.

[116] R. Thewes. Shock-wave process for the removal of mould residues and cores for the production of castings. CP + T - Casting Plant and Technology, 1 (1993) 2-8.

[117] V. Venugopalan, A. Guerra, K. Nahen, A. Vogel. Role of laser-induced plasma formation in pulsed cellular microsurgery and micromanipulation. Phys. Rev. Lett., 88 (2002) 0781031-0781034.

[118] B. Wolfrum, T. Kurz, R. Mettin, W. Lauterborn. Shock wave induced interaction of microbubbles and boundaries. Phys. Fluids, 15 (2003) 2916-2922.

[119] B. Wolfrum, T. Kurz, O. Lindau, W. Lauterborn. Luminescence of transient bubbles at elevated ambient pressures. Phys. Rev. E, 64 (2001) 043061-043065.

[120] F. Docchio, P. Regondi, M.R.C. Capon, J. Mellorio. Study of the temporal and spatial dynamics of plasmas induced in liquids by nanosecond Nd:YAG laser pulses. Appl. Optics, 27 (1988) 3661-3674.

[121] D.J. Stolarski, J. Hardman, C.G. Bramlette, G.D. Noojin, R.J. Thomas, B.A. Rockwell, W.P. Roach. Integrated light spectroscopy of laser-induced breakdown in aqueous media. SPIE Proc., 2391 (1995) 100 - 109.

[122] J. Noack, A. Vogel. Single-shot spatially resolved characterization of laser-induced shock waves in water. Appl. Optics, 37 (1998) 4092 - 4099.

[123] J. Staudenraus, W. Eisenmenger. Fibre-optic probe hydrophone for ultrasonic and shock wave measurement in water. Ultrasonics, 31 (1993).

[124] Manual of the optical fiber hydrophone FOPH 300, University of Stuttgart, 1997. 
[125] W. Lauterborn. High-speed photography of laser-induced breakdown in liquids. In: Paget, E. Laviron (Association Nationale de la Recherche Technique), Eds., Actes du X Xeme Congr. Int. de Cinematographie, 1972, 306-309.

[126] M. Strasberg. The pulsation frequency of nonspherical gas bubbles in liquids. $J$. Acoust. Soc. Am., 25 (1953).

[127] B. Wolfrum, R. Mettin, T. Kurz, W. Lauterborn. Observations of pressure-waveexcited contrast agent bubbles in the vicinity of cells. Appl. Phys. Lett., 81 (2002) 5060-5062.

[128] H.M. Jones, E.E. Kunhardt. Pulsed dielectric breakdown of pressurized water and salt solutions. J. Appl. Phys., 77 (1995) 795-805.

[129] M. Lokhandwalla, B. Sturtevant. Mechanical haemolysis in shock wave lithotripsy (SWL): I. Analysis of cell deformation due to SWL flow-fields. Phys. Med. Biol., 46 (2001) 413-437.

[130] C.-D. Ohl, R. Ikink. Shock-wave-induced jetting of micron-size bubbles. Phys. Rev. Lett., 90 (2003) 214502-4.

[131] L.A. Crum, R.A. Roy, M.A. Dinno, C.C. Church, R.E. Apfel, C.K. Holland, S.I. Madanshetty. Acoustic cavtiation produced by microsecond pulses of ultrasound: A discussion of some recent results. J. Acoust. Soc. Am., 91 (1992) 1113-1119.

[132] R. Mettin, B. Wolfrum, B. Siegmund, H. Kijewski. Druckwelleninduzierte Kavitation in der Wundballistik. In: M. Vorländer, Ed., Fortschritte der Akustik DAGA'03 (Aachen), 2003, 839-840.

[133] B. Sigmund. Kavitationseffekte in der Wundballistik. Dissertation, Georg-AugustUniversität zu Göttingen, to appear in 2004.

[134] K. Sellier. Schußwaffen und Schußwirkungen I. Schmidt-Römhild, Lübeck, 1969.

[135] K. Sellier. Schußwaffen und Schußwirkungen II. Schmidt-Römhild, Lübeck, 1977.

[136] Y. Liu, R. Guo, B. Wu, S. Li, D. Wang. Pressure variation in temporary cavities trailing three different projectiles penetrating water and gelatin. Journal of Trauma, 28 (1998) 9-16. 
[137] C. Schyma, P. Schyma. Das Verletzungspotential von Kartuschenmunition im Gelatinemodell. Rechtsmedizin, 9 (1999) 210-214.

[138] O. Lindau. Dynamik und Lumineszenz lasererzeugter Kavitationsblasen. Diplomarbeit, 1998.

[139] A. Suneson, H.-A. Hansson, T. Seeman. Central and peripheral nervous damage following high-energy missile wounds in the thigh. J. Traum., 28 (1988) 197-203.

[140] C.-D. Ohl, B. Wolfrum. Detachment and sonoporation of adherent HeLa-cells by shock wave-induced cavitation. Biochim. Biophys. Acta, 1624 (2003) 131-138.

[141] H.G. Flynn, C.C. Church. A mechanism for the generation of cavitation maxima by pulsed ultrasound. J. Acoust. Soc. Am., 76 (1984) 505-512.

[142] O.A. Sapozhnikov, V.A. Khokhlova, M.R. Bailey, J.C. Williams, J.A. McAteer, R.O. Cleveland, L.A. Crum. Effect of overpressure and pulse repetition frequency on cavitation in shock wave lithotripsy. J. Acoust. Soc. Am., 112 (2002) 1183-1195.

[143] L. Junge, C.-D. Ohl, B. Wolfrum, M. Aurora, R. Ikink. Cell detachment method using shock-wave-induced cavitation. Ultrasound Med. Biol., 29 (2003) 1769-1776.

[144] F. Calliada, R. Campani, O. Bottinelli, A. Bozzini, M.G. Sommaruga. Ultrasound contrast agents: basic principles. Eur. J. Radiol., 27 (1998) 157-160.

[145] N. Kasahara, A.M. Dozy, Y.W. Kan. Tissue-specific targeting of retroviral vectors through ligand-receptor interactions. Science, 266 (1994) 1373-1376.

[146] W.T. Shi, F. Forsberg, A. Tornes, J. Ostensen, B.B. Goldberg. Destruction of contrast microbubbles and the association with inertial cavitation. Ultrasound Med. Biol., 26 (2000) 1009-1019. 


\section{Lebenslauf}

\section{Angaben zur Person:}

$\begin{array}{ll}\text { Name: } & \text { Bernhard Lars Wolfrum } \\ \text { Geburtstag und -ort: } & \text { 27. Mai 1975 in Bonn } \\ \text { Familienstand: } & \text { verheiratet mit Silke Wolfrum (geb. Stange) } \\ \text { Kinder: } & \text { Ein Sohn: Lennart Wolfrum } \\ \text { Staatsangehörigkeit: } & \text { deutsch }\end{array}$

\section{Schulbildung:}

$1981-1982$

Montessori Grundschule in Bonn

$1982-1985$

Claus-Rixen Grundschule in Altenholz

$1985-1991$

Gymnasium Altenholz

$1991-1992$

$1992-1994$

06/1994

Washburn Highschool, Minnesota, MN, USA

Gymnasium Altenholz

Allgemeine Hochschulreife

\section{Wehrdienst:}

$10 / 94-02 / 95$

Grund- und Fernmeldeausbildung in Eckernförde bzw. Schleswig

$03 / 95-09 / 95$

Funker auf einem Minensuchboot

\section{Studium:}

$10 / 1995-06 / 1997$

Grundstudium Physik an der Georg-August-Universität Göttingen

$06 / 1997$

Vordiplom

09/1997-07/1998

Studium an der University of California Santa Barbara

seit $10 / 1998$

Studium an der Georg-August-Universität Göttingen

$01 / 2000-11 / 2000$

Diplomand am Dritten Physikalischen Institut der Universität Göttingen in der Arbeitsgruppe „Nichtlineare Physik“

$11 / 2000$

Diplom

seit $01 / 2001$

Promotion und wissenschaftlicher Mitarbeiter am Dritten Physikalischen Institut der Universität Göttingen bei Prof. Dr. W. Lauterborn

Aug. 2002 - Okt. 2002 Gastwissenschaftler an der University of Twente in der Arbeitsgruppe „Physics of Fluids“ 